Dritter Beratungsgegenstand:

\title{
Risikosteuerung durch Verwaltungsrecht: Ermöglichung oder Begrenzung von Innovationen?
}

1. Bericht von Prof. Dr. Arno Scherzberg, Erfurt*

Inhalt

Seite

I. Der Ausgangsbefund: das Phänomen des Risikos . . . . . . . 216

II. Begriff und Theorie des Risikos . . . . . . . . . . . . . . . 219

1. Das Risiko erster Ordnung . . . . . . . . . . . . . 219

2. Das Risiko zweiter Ordnung . . . . . . . . . . . . . . 220

3. Der Risikobegriff des Risikoverwaltungsrechts . . . . . 223

III. Reflexive Risikosteuerung: Theoretische Grundlagen

und Wirkungsbezüge . . . . . . . . . . . . . . 225

1. Recht als Instrument der Risikosteuerung . . . . . . . . . 225

2. Risiko und Innovation als Gegenstand rechtlicher

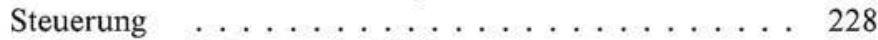

a) Die Konstituierung des Risikos . . . . . . . . . . . . 228

b) Risikobewertung und Risikovergleich . . . . . . . . 230

c) Die Bewertung von Innovationen . . . . . . . . 232

d) Risikosteuerung als innovativer Prozess . . . . . . . 234

e) Risikosteuerung als Steuerung von Innovationen . . . 235

IV. Das Recht der reflexiven Risikosteuerung . . . . . . . . . . 238

1. Gemeinschaftsrechtliche Grundlagen . . . . . . . . . 238

2. Verfassungsrechtliche Grundlagen ... . . . . . . . . 240

a) Zielsetzung und Voraussetzungen . . . . . . . . . . . 240

b) Modalitäten und Instrumente . . . . . . . . . . . . 243

3. Die Risikosteuerung im Risikoverwaltungsrecht . . . . . . 245

a) Die Risikoreduktion . . . . . . . . . . . . . . . 245

(aa) Das Regelungsmodell . . . . . . . . . . . . 245

(bb) Die Maßstabsbildung im Verfahren ....... 246

\footnotetext{
* Meinem Mitarbeiter Matthias Mayer danke ich für zuverlässige Recherchen und weiterführende Ideen.
} 
(cc) Die Verteilung der Beweislast . . . . . . . . 247

(dd) Die Zuweisung des Risikos . . . . . . . . . . . 249

(ee) Die Dynamisierung der Regulierung . . . . . 251

b) Die Risikoprävention . . . . . . . . . . . 252

(aa) Das Regelungsmodell . . . . . . . . . 252

(bb) Die Instrumente . . . . . . . . . . . . 252

(1) Das ökologische Erforderlichkeitsgebot . . . 252

(2) Das Stoff- und Energiestrommanagement . 253

(3) Die Risikohaftung . . . . . . . . . 254

(4) Die Risikokommunikation . . . . . . 255

V. Ausblick: Rechtliche Ungewissheitsbewältigung und ihre

Rückwirkungen auf das Recht . . . . . . . . . . . 256 
„Die Notwendigkeit zu entscheiden reicht weiter als die Fähigkeit zu erkennen" (Immanuel Kant)

„Es kann sich tatsächlich erweisen, daß es die weitaus schwierigste und nicht die unwichtigste Aufgabe für die menschliche Vernunft ist, ihre eigenen Grenzen rational zu erfassen" (Friedrich v. Hayek)

\section{Der Ausgangsbefund: das Phänomen des Risikos}

In einer Gesellschaft ohne Glauben an Schicksal oder Fügung erscheint die Zukunft prinzipiell gestaltbar, aber auch in einer beunruhigenden Weise unsicher und offen. ${ }^{1}$ Dies gilt trotz oder sogar wegen ihrer wissenschaftlich-technischen Errungenschaften, denn diese erhöhen zwar die Verarbeitungsfähigkeit und Wirkungsmacht zivilisatorischer Systeme, steigern aber auch deren Fehleranfälligkeit ${ }^{2}$ und Schadenspotentiale. ${ }^{3}$

Jüngstes Beispiel ist die Nanotechnologie. Nanopartikel sind wegen ihrer geringen Größe und vergleichsweise großen Oberfläche besonders reaktionsfreudig und vielseitig einsetzbar. Produkte der Energie-, Umwelt- und Informationstechnik, sogar neue Verfahren zur Krebsbehandlung gehören zu ihren Anwendungsbereichen. Wegen ihrer Winzigkeit können sie allerdings auch leicht über Haut und Atmung in den menschlichen Organismus gelangen und dort Probleme für das Immunsystem, das Lungengewebe und die Blutgefäße verursachen. Was ihre „Freisetzung" für die Umwelt bewirkt, ist offenbar noch gänzlich unerforscht. Erste Umweltschutzorganisationen fordern bereits ein Moratorium der Technologie und die britische Regierung beauftragte unlängst die Royal Society mit einer Studie über ihre Folgen. ${ }^{4}$ In Deutschland hatte kürzlich der VGH Baden-Württemberg über eine Anlage zur Herstellung von Nanopulver zu entscheiden. 5

\footnotetext{
1 Meyer in: Gesamtverband der deutschen Versicherungswirtschaft e.V. (Hrsg.) Risiko - Wieviel Risiko braucht die Gesellschaft, 1998, 148ff.

${ }^{2}$ Dazu Kornwachs in: Banse (Hrsg.) Risikoforschung zwischen Disziplinarität und Interdisziplinarität, 1996, 73, 78 .

${ }^{3}$ S. etwa Schulze-Fielitz in: Schulte (Hrsg.) Handbuch des Technikrechts, 2003, 443, 448.

${ }^{4}$ Focus 36/2003, 80f.; ferner www.nanonet.de.index.phb3.

${ }^{5}$ VGH BW VBIBW 2002, 436f.
} 
Die moderne Gesellschaft steigert mit ihrem technikbasierten Handlungspotential also offenbar zugleich Sicherheit und Ungewissheit. ${ }^{6}$ Diese paradoxe Entwicklung findet im Konzept von Chance und Risiko Ausdruck. ${ }^{7}$ Es bezeichnet die Relation möglicher Gewinne und Verluste als Folgen einer Entscheidung und betrachtet diese damit vor dem Hintergrund der Ungewissheit ihrer Konsequenzen. ${ }^{8}$ Im Risikobegriff sollen diese Konsequenzen berechenbar und zurechenbar gemacht, die Ungewissheit also kalkulier ${ }^{9}$ und in rationale Entscheidungen transformiert werden. ${ }^{10}$

Rationales Handeln ist indes, in der hier gemeinten Form der Zweckrationalität, auf empirisch begründete Annahmen über die Eignung eines Mittels zur Erreichung des Zwecks angewiesen," setzt also eine „Herrschaft kraft Wissens" ${ }^{\text {12 }}$ voraus, von der für die meisten Steuerungsprobleme der "Risikogesellschaft"13 nicht die Rede sein kann. Rationalität muss unter Ungewissheitsbedingungen deshalb neu definiert werden. ${ }^{14}$ Die Notwendigkeit der fortlaufenden Aktualisierung des Risikowissens ist dabei unbestritten. ${ }^{15}$ Das Ziel der Risikovorsorge, wie es heute aufgrund völker-16,

${ }^{6}$ Dazu Banse in: ders. (Fn. 2), 15, 17; Beck in: ders./Giddens/Lash (Hrsg.) Reflexive Modernisierung, 1996, 19, 55; G.-P. Calliess Prozedurales Recht, 1999, 58.

7 Grundlegend Kloepfer UTR 5 (1988), 31 ff., Bechmann KritV 74 (1991), 212, 214; zur historischen Entwicklung des Risikobegriffs Banse in: ders. (Fn. 2), 15, $23 \mathrm{ff}$.

${ }^{8}$ Holzheu in: Bayerische Rück (Hrsg.) Risiko ist ein Konstrukt, 1993, 263, 265; ähnlich Luhmann Soziologie des Risikos, 1991, 235.

9 Giddens Modernity and Self-Identity, 1991, 111 f., $114 \mathrm{ff}$., $133 \mathrm{f}$.; Beck Weltrisikogesellschaft revisited, www.scienceandfiction.de/04/pdf/013Beck.pdf.

${ }^{10}$ Banse in: ders. (Fn. 2), 28f.; Evers Kommune 6/1989, 33, 34.

" Acham in: Schnädelbach (Hrsg.) Rationalität, 1984, 32, 51; Cohen „Rationalität", in: Speck (Hrsg.) Handbuch wissenschaftstheoretischer Begriffe III, 1980, 531 ff.

12 Weber Wirtschaft und Gesellschaft, 1972, 129.

${ }_{13}$ Zum Begriff Beck Risikogesellschaft - Auf dem Weg in eine andere Moderne, 1986, $25 \mathrm{ff}$.; Steinberg Der ökologische Verfassungsstaat, 1998, $23 \mathrm{ff}$.

${ }_{14}$ Bon $\beta$ Vom Risiko: Unsicherheit und Ungewissheit in der Moderne, 1995, 291 ff.; ferner Gigerenzer in: Engel/Halfmann/Schulte (Hrsg.) Wissen-Nichtwissen-Unsicheres Wissen, 2002, 161, 182; Priddat in: Banse (Fn. 2), 105 ff.

is S. etwa Di Fabio FS Ritter, 1997, 807, $820 \mathrm{ff}$.

${ }^{16} \mathrm{Vgl}$. nur Grundsatz 15 der Rio-Deklaration für Umwelt und Entwicklung (1992) ILM 31 (1992), 874 ff.; Art. 2 des Übereinkommens zum Schutz und zur Nutzung grenzüberschreitender Wasserläufe und internationaler Seen (1992) BGBI. II, 1994, 2334; Art. 3 des Rahmenübereinkommens der UN über Klimaveränderungen (1992), BGBI. II, 1993, 1784; weitere Beispiele bei Beyerlin Umweltvölkerrecht, 2000, Rn. 187, 193, 206, 293; Sand, Human and Ecological Risk Assessment 6 (2000), 445 ff.; ferner die Mitteilung der Kommission „Die Anwendung des Vorsorgeprinzips“, KOM (2000) endg., 1, $10 \mathrm{ff}$. 
europa-17 und verfassungsrechtlicher ${ }^{18}$ Festlegungen die Risikosteuerung bestimmt, fordert aber eine zeitnahe und antizipative und deshalb in Voraussetzungen und Wirkungen ungewisse Risikoregulierung. Unter diesen Umständen kann sich das Recht nicht mit der Forderung nach zusätzlichem Wissenserwerb begnügen, ${ }^{19}$ sondern muss das Handeln unter Ungewissheitsbedingungen als solches annehmen und strukturieren. ${ }^{20}$

Dabei gerät in den Blick, dass die Folgen rechtlicher Risikosteuerung nicht weniger ungewiss sind als die Risiken selbst. ${ }^{21}$ FCKW, PCB und Asbest $^{22}$ sind Beispiele für die Risiken der Strategie, bekannte Gefahrenquellen durch unbekannte zu ersetzen. ${ }^{23}$ „Some cures are worse than the disease" sagt der amerikanische Verwaltungsrechtler Cass Sunstein.24 Unter Ungewissheitsbedingungen lässt sich das freilich im Vornherein kaum feststellen. Rechtliche Risikosteuerung ist deshalb immer eine „tragic

17 Vgl. etwa Art. 174 Abs. 2 S. 2 EGV; Art. 4 Abs. 1 RL 2001/18 EG über die absichtliche Freisetzung genetisch veränderter Organismen in die Umwelt und zur Aufhebung der RL 90/220 EWG, ABI. L 106/1; Art. 7 Abs. 1 VO (EG) 178/2002 zur Festlegung der allgemeinen Grundsätze des Lebensmittelrechts ABl. L 31/1; Art. 3, 4 VO (EWG) 793/93 zur Bewertung und Kontrolle der Umweltrisiken chemischer Altstoffe, ABI. L 84/1; Art. 3 Abs. 1 lit. a RL 96/61 EG über die integrierte Vermeidung und Verminderung der Umweltverschmutzung, AB1. L 257/26.

${ }^{18} \mathrm{Zu}$ den grundrechtlichen Schutzpflichten und Art. 20a GG als Präventionsgebot Steinberg (Fn. 13), $91 \mathrm{ff}$; ferner unten IV.2.

${ }^{19}$ Dabei ist auch in Rechnung zu stellen, dass die Erarbeitung abgesicherter Wissensbestände häufig mit der Entdeckung neuer, noch größerer Unbestimmtheiten einher geht, vgl. Fleck Entstehung und Entwicklung einer wissenschaftlichen Tatsache, 1980, $109 \mathrm{ff}$. und Weingart Die Stunde der Wahrheit, 2001, 20, und dass zusätzliches Wissen deshalb nicht notwendig zu angemesseneren Entscheidungen führt und die Kosten einer Verbesserung der Informationslage mithin weit höher sein können als der Ertrag. Dazu auch Weingart ebd., 337; Wegner Marktkonforme Umweltpolitik zwischen Dezisionismus und Selbststeuerung, 1994, 15; gerade dies kann man freilich in der Entscheidungssituation regelmäßig nicht erkennen, vgl. Tietzel Wirtschaftstheorie und Unwissen, 1985, 19f.

${ }^{20} \mathrm{Damm} /$ Hart KritV 70 (1987), 183, 185; Ladeur Das Umweltrecht der Wissensgesellschaft, 1995, 40ff., 79 ff., 118 ff.; Spiecker gen. Döhmann in: Lege (Hrsg.) Gentechnik im nicht-menschlichen Bereich - was kann und was sollte das Recht regeln?, 2001, 51, 77; gleiches gilt für den außerrechtlichen Umgang mit dem Risiko, Bonß (Fn. 14), 303f.; Japp Risiko, 2000, 30f.

21 Wienerin: Graham/Wiener (Hrsg.) Risk versus Risk, 1997, 193, 196ff.; Wolf in: Roßnagel/Neuser (Hrsg.) Reformperspektiven im Umweltrecht, 1996, 57, 95.

${ }_{22} \mathrm{Zu}$ Entwicklungsgeschichte und Problemen der FCKW vgl. European Environment Agency (Hrsg.) Late lessons from early warnings, 2001, 76 ff.; Der Rat von Sachverständigen für Umweltfragen (Hrsg.) Umweltgutachten 2002, Tz. 417 ff.; Fallstudien zu Asbest und den chlorierten aromatischen Kohlenwasserstoffen (PCB) in European Environment Agency, ebd., $52 \mathrm{ff} ., 64 \mathrm{ff}$.

${ }^{23}$ S. etwa Wahl/Appel in: Wahl (Hrsg.) Prävention und Vorsorge, 1995, 1, 7.

${ }^{24}$ Sunstein in: Graham/Wiener (Fn. 21), VII, VIII. 
choice ${ }^{25}$ und selbst riskant. ${ }^{26}$ Risiko tritt dem Recht mithin in zwei Erscheinungsformen entgegen: als Schadenspotential in der von ihm regulierten Umwelt ${ }^{27}$ und als mögliche Irrtumskosten der rechtlichen Regulierung selbst.

\section{Begriff und Theorie des Risikos}

Eine Entscheidung ist riskant, wenn sie möglicherweise unerwünschte Folgen zeitigt. ${ }^{28}$ Sie verliert dieses Prädikat, wenn man ihre Folgen vollständig kennt. ${ }^{29}$ Diese Bedingung ist indes praktisch nie erfüllt. Wenn man von Gewissheit der Folgen und damit gegebenenfalls auch von Sicherheit rechtlich geschützter Güter spricht, und von Chance und Risiko als der anderen Seite der Unterscheidung, handelt es sich deshalb nicht um empirisch-vorfindliche Phänomene, sondern um aus kulturellen oder wissenschaftlichen Wertungen gewonnene Konstrukte. ${ }^{30}$ Für die Zwecke rechtlicher Risikoverarbeitung sind zwei solcher Konstrukte erforderlich, die hier „Risiko erster Ordnung“ und „Risiko zweiter Ordnung" genannt werden sollen.

\section{Das Risiko erster Ordnung}

Das Risiko erster Ordnung bezeichnet den Erwartungswert eines Schadens. Diesen errechnet die Entscheidungstheorie ${ }^{31}$ als Produkt von Schadenshöhe und Eintrittswahrscheinlichkeit und macht damit die nachteiligen Folgen ökonomischer Entscheidungen mit ihrem erwarteten Nutzen vergleichbar.

25 Calabresi/Bobbilt Tragic choices, 1978, 26; Steinberg(Fn. 13), 92.

${ }^{26}$ Ladeur (Fn. 20), 118; Wolfin: Bora (Hrsg.) Rechtliches Risikomanagement, 1999, 65, 84; für das Arzneimittelrecht Krücken in: Hiller/Krücken (Hrsg.) Risiko und Regulierung, 1997, 116, 118.

27 Typologie des Risikos insoweit bei Scherzberg in: Engel/Halfmann/Schulte (Fn. 14), $113,117$.

${ }^{28}$ Wissenschaftlicher Beirat der Bundesregierung Globale Umweltveränderungen/ WBGU (Hrsg.) Welt im Wandel: Jahresgutachten 1998, 1999, 35.

29 S. etwa Peine UTR 43 (1998) 111, 112.

30 Holzheu (Fn. 8), 264; WBGU (Fn. 28), 38; Heidenescher Die Beobachtung des Risikos, 1999, 61 ff.; Luhmann Soziologische Aufklärung 5, 1990, 134; Di Fabio in: Banse (Fn. 2), 133; Krohn/Krücken, in: dies. (Hrsg.) Riskante Technologien 1993, 9, $10 \mathrm{ff.;}$ zum kulturwissenschaftlichen Aspekt Renn in: Chakraborty/Yadigaroglu (Hrsg.) Ganzheitliche Risikobetrachtung, 1991, 06-1, 06-14ff.; zum Konstrukt der Wahrscheinlichkeit als Element des Risikos vgl. Sinn Ökonomische Entscheidungen bei Ungewissheit, 1980, 19.

${ }^{31}$ Grundlegend Knight Risk, Uncertainty and Profit, 1921, 22 ff, $197 \mathrm{ff}$. 
Ulrich Beck verdanken wir indes den Hinweis, dass ein solcher Risikobegriff mögliche Gewissheitsdefizite bei der Schadensprognose vernachlässigt und so über die Möglichkeit einer Fehleinschätzung hinwegtäuscht. ${ }^{32}$ Das ist unproblematisch, solange sich die Risikokalkulation wie bei Versicherungsprämien und Glücksspielchancen, den historischen Anwendungsfeldern des entscheidungstheoretischen Risikos, ${ }^{33}$ auf mathematisch errechenbare Wahrscheinlichkeiten und das Gesetz der großen Zahl stützen kann, das unerwartete Entwicklungen in Einzelfällen kompensiert. ${ }^{34}$ Gleiches dürfte gelten, soweit das Recht auf das Erfahrungswissen von Vollzugsbeamten und das technische Fachwissen von Ingenieuren zurückgreift, dem immerhin auch eine Vielzahl von Anwendungsfällen zugrunde liegt. ${ }^{35}$

\section{Das Risiko zweiter Ordnung}

Die Folgen des Einsatzes moderner Technik sind allerdings in ihrer Komplexität und Singularität vielfach weder einer statistischen noch einer erfahrungsbasierten Beurteilung zugänglich. ${ }^{36}$ Sie sind meist weder kausal sicher zurechenbar noch raum-zeitlich begrenzt. Als „schleichende Katastrophen" bleiben sie zudem oft unerkannt oder unterschätzt und werden erst im Verlauf ihrer Anwendung - bei den FCKW immerhin erst nach 40 Jahren - in ihren verheerenden Wirkungen deutlich. ${ }^{37}$ Angesichts der Höhe der Schadenspotentiale muss das Recht möglichst verhindern, dass auf diese Weise Erfahrung gewonnen wird.

\footnotetext{
${ }^{32}$ Beck Gegengifte, 1988, 103 ff.; s. auch Priddat in: Banse (Fn. 2), 105 f.; Reese-Schäfer in: Dicke (Hrsg.) Politisches Entscheiden, 2001, 27, 30ff.; Rehmann-Sutter in: Skorupinski/Ott (Hrsg.) Ethik und Technikfolgenabschätzung, 2001, $80 \mathrm{ff}$.

${ }^{33}$ Zum Versicherungswesen Banse in: ders. (Fn. 2), $28 \mathrm{ff}$.

${ }^{34}$ Jedenfalls dann, wenn sich der Entscheider am Durchschnittserfolg je Realisation orientiert; näher Laux Entscheidungstheorie, 5. Aufl. 2003, $147 \mathrm{ff}$.

${ }_{35}$ Vornehmlich Karl-Heinz Ladeur hat darauf hingewiesen, dass der klassische Gefahrenbegriff ein Operieren unter Ungewissheit dadurch ermöglicht und begrenzt hat, dass er auf eine hinreichend sichere Prognose der Schadensneigung durch einen durchschnittlich kundigen Beobachter abstellte und die Berechenbarkeit der fraglichen Abläufe aufgrund von Erfahrungswissen voraussetzte; dazu Ladeur (Fn. 20), 9 ff.; ebenso Banse in: ders. (Fn. 2), 37f.; Hoffmann-Riem in: ders./Schmidt-Aßmann/Schuppert (Hrsg.) Reform des Allgemeinen Verwaltungsrechts, 1993, 115, 166f.; C. Calliess Rechtsstaat und Umweltstaat, 2001, $155 \mathrm{ff}$.

${ }^{36}$ Banse in: ders. (Fn. 2), 33f.

${ }^{37}$ Böhret Folgen: Entwurf für eine Politik gegen schleichende Katastrophen, 1990; Bundesministerium für Umwelt, Naturschutz und Reaktorsicherheit (Hrsg.) Umweltpolitik, Umweltgutachten 2000 des Rates von Sachverständigen für Umweltfragen, 2000, 17.
} 
Dazu lassen sich freilich im Rahmen der Gefahrenabwehr, dem Schema der Bewältigung der Risiken erster Ordnung, keine angemessenen Instrumente finden. ${ }^{38}$ Weder die Beschränkung auf die Gefahrerforschung, noch eine unbegrenzte Absenkung der Gefahrenschwelle oder der Verzicht auf Schadensbegrenzung wären angemessene Reaktionen auf das Fehlen verlässlicher Prognosen. ${ }^{39}$ Soll die Steuerungsleistung des Rechts hier nicht enden, muss es von einer erfahrungsbasierten auf eine ungewissheitsbasierte Strategie normativer Zukunftsbewältigung übergehen. ${ }^{40}$ Im Wege der Vorsorge trägt es dann dem Risiko Rechnung, Risiken nicht rechtzeitig zu erkennen. ${ }^{41}$

Vorsorge muss dafür auf den jeweiligen Stand der Wissenschaft rekurrieren. Dabei fließen die Ergebnisse von Simulationen, Extrapolationen und Modellrechnungen und damit auch schwach verifizierte Hypothesen in die Risikoprognose ein. ${ }^{42}$ So hat das BVerfG im Kalkar-Beschluss die Einbeziehung aller "vertretbaren" Erkenntnisse gefordert und das BVerwG im Whyl-Urteil auf die Notwendigkeit hingewiesen, auch „bloße theoretische Überlegungen und Berechnungen" für das Risikokalkül in Betracht zu ziehen. ${ }^{43} \mathrm{Je}$ weiter sich rechtliches Risikomanagement damit aber in die Ungewissheit hinein ausweitet, desto höher wird, worauf vor

\footnotetext{
38 Ladeur (Fn. 20), $69 \mathrm{ff}$.

${ }^{39}$ Karthaus Risikomanagement und ordnungsrechtliche Steuerung, 2001, 60ff.; Köck Maßstäbe der Risikobewertung im Umweltrecht, n.v. Habilitationsschrift, 2000, 269.

${ }^{40}$ In diesem Sinn die Europäische Umweltagentur nach Auswertung von 14 Fallstudien zum Risikomanagement der letzten 100 Jahre: „Acknowledge and respond to ignorance ... uncertainty and risk in technology appraisal and public policy-making. ... To be alert to - and humble about - the potential gaps in those bodies of knowledge that are included in our decision-making is fundamental." Dazu vgl. European Environment Agency (Fn. 22), 168f.; vgl. ferner Gill/Bizer/Roller Riskante Forschung, 1998, 18f.; Herdegen/Dederer in: Eberbach/Lange/Ronellenfitsch, GenTR, $\$ 15$ GenTG, Rn. 80 Fn. 169; Wolf in: Bora (Fn. 26), 65, $77 \mathrm{ff}$.

41 Gill ÖZP 27 (1998), 29, 33; C. Calliess DVBI 2001, 1725, 1727; Scherzberg VerwArch 84 (1993), 484, 498.

42 Köck (Fn. 39), 58, 152 ff.; vgl. auch WBGU (Fn. 28), 4; Priddat in: Banse (Fn. 2), 105, 108; zum Beispiel der Reaktorsicherheitsstudie WASH 1200 aus dem Jahre 1975 vgl. Nowitzki in: Bechmann, Risiko und Gesellschaft, 2. Aufl. 1997, 125, 130; zur Subjektivität der Schlussfolgerung aus Schätzungen, Simulationen und Experimenten vgl. auch Eisenführ/Weber Rationales Entscheiden, 2. Aufl. 1994, $149 \mathrm{ff}$.

43 BVerfGE 49, 89, 136; BVerwGE 72, 300, 315; aus neuerer Zeit BVerwGE 104, 36, $51 ; 106,115,121$. Dabei bleibt noch unberücksichtigt, dass die Theorie - nicht nur im Bereich moderner Technologie vielfach aus der zu beurteilenden Anwendung erst folgt. Dazu vgl. Kleindick Wissenschaft und Freiheit in der Risikogesellschaft, 1998, $102 \mathrm{ff}$.
} 
allem US-amerikanische Autoren hinweisen, das Risiko zweiter Ordnung, ${ }^{44}$ die Gefahr der Fehleinschätzung und Fehlsteuerung der Gefahr. ${ }^{45}$

Um ihr zu begegnen, muss das Recht seine eigenen Wirkungen antizipieren. ${ }^{46}$ Es wird damit reflexiv. ${ }^{47}$ Risikosteuerung beschränkt sich dann nicht mehr auf den Umgang mit den nachteiligen Folgen eines äußeren Geschehens, sondern erstreckt sich auch auf die nachteiligen Folgen der rechtlichen Normierung. ${ }^{48}$ Das erfordert eine Einschätzung der Zwecke, Beschränkungen, Nebenfolgen, Ungewissheiten und Irrtumskosten eines Regelungsprojekts und eine Entscheidung über seine Verantwortbarkeit vor dem Hintergrund unzureichenden Wissens. ${ }^{49}$ Die Grenzen des wis-

44 Dazu Wiener/Graham in: dies. (Fn. 21), 226, 228ff.; Warren/Marchant Ecology Law Quaterly 20 (1993), 379 ff.; Adler Texas International Law Journal 35 (2000), 173, $194 \mathrm{ff}$;; ferner die Beiträge von Morrow und Jaffe in: Hunziker/Jones (Hrsg.) Product Liabilits and Innovation, 1994, 23, 27 und 120, 126; ferner Williamson/Hulpke Zeitschrift für Umweltchemie und Ökotoxologie (UWSF) 12 (2000), 91, 92 ff.; Köck/Hansjürgens GAIA 11 (2002), 42f.; Cross Washington and Lee Law Review 53 (1996), 851, 859ff.; Sunstein Risk and Reason, 2002, $133 \mathrm{ff}$; Miller/Conko Policy Review 107 (2001), $25 \mathrm{ff}$.

45 Scherzberg in: Engel/Halfmann/Schulte (Fn. 14), 113, 134; ferner Rehmann-Sutter (Fn. 32), 80 .

46 Trute in: Riedel (Hrsg.) Risikomanagement im öffentlichen Recht, 1997, 55, 56.

47 Preuß $\beta$ in: Grimm (Hrsg.) Staatsaufgaben, 1994, 523, 542; zum Spielraum der Verwaltung zur Folgenberücksichtigung vgl. Scherzberg Die Öffentlichkeit der Verwaltung, 2000, 132f.; Hoffmann-Riem in: ders./Schmidt-Aßmann, Innovation und Flexibilität des Verwaltungshandelns, 1994, 8, 24f.; indirekt auch Sendler FS Simon, 1987, 113, $136 \mathrm{ff}$.; allgemein zum Begriff der Reflexivität Giddens Konsequenzen der Moderne, 1995, $52 \mathrm{ff}$.; Wagner/Haffner UTR 49 (1999), 93, 99. Die Antizipation und Integration der Folgen des eigenen Handelns ist auch Ziel des Steuerungsmodells des „reflexiven Rechts“, dazu mit Beispielen Schmidt-Preuß VVDStRL 56 (1997), 160, 192 ff.; Teubner/Willke ZfRSoz 1984, $4,19 \mathrm{ff}$.

48 Schmidt-Preuß in: Kloepfer (Hrsg.) Technikumsteuerung als Rechtsproblem, 2002, 119, 127; Köck (Fn. 39), 269; Di Fabio Jura 1996, 566, 573; Krohn/Krücken (Fn. 30), 23; Williamson/Hulpke(Fn. 44), 95; solche können gerade aus der Beseitigung einer Risikoquelle erwachsen; so wird heute ein Sicherheitsverlust gerade wegen des Ausstiegs aus der Kernenergie befürchtet, vgl. dazu Bohne NVwZ 1999, 1, 5; insoweit kann zusätzliches Risikomanagement erforderlich sein. Risikosteuerung zweiter Ordnung unterscheidet sich also nicht nur auf der Mittelebene, sondern auch in seinen Zielen von der Gefahrenabwehr; s. bereits Scherzberg in: Engel/Halfmann/Schulte (Fn. 14), 113, 135f.; a. A. für die Risikovorsorge Lübbe-Wolff in: Bizer/Koch (Hrsg.) Sicherheit, Vielfalt, Solidarität, Symposium für Denninger, 1998, 47, 52.

49 S. etwa EuG Rs. T-70/99 v. 11. 9. 2002 (Alpharma/Rat), Slg. 2002, II-3495, Rn. 174f.; dazu auch bereits Scherzberg (Fn. 41), 497 ff.; Franzius Die Herausbildung der Instrumente indirekter Verhaltenssteuerung im Umweltrecht der Bundesrepublik Deutschland, 2000, 92ff.; Schmidt-Aßmann Das allgemeine Verwaltungsrecht als Ordnungsidee, 1998, 3/73; Seiler in: Banse (Fn. 2), 145, 162; Wahl/Appel (Fn. 23), 98; Berg Die verwaltungsrechtliche Entscheidung bei ungewissem Sachverhalt, 1980, 298; Di Fabio (Fn. 15), 824f.; Williamson/Hulpke (Fn. 44), 95. 
senschaftlichen Erkenntnisvermögens bezeichnen dabei nicht notwendig den Endpunkt, sondern geben u.U. auch Anlass zu rechtlicher Regulierung. ${ }^{50}$ Das Wissen um das Nichtwissen kann etwa eine verdachtsunabhängige Prävention ${ }^{51}$ oder die Einführung von Risikoindikatoren als Entscheidungsheuristiken nahe legen, ${ }^{52}$ die auf der Grundlage geringerer Erkenntnissicherheit Erfolg versprechen. ${ }^{53}$ Hohe Irrtumskosten werden regelmäßig die Grenzen der Vorsorge unter Ungewissheit indizieren: Restrisiko ist dasjenige Risiko, bei dem die Risiken der Risikosteuerung (durch Vorsorge) überwiegen.

\section{Der Risikobegriff des Risikoverwaltungsrechts}

Soweit das heutige Verwaltungsrecht wie etwa in $\S 6$ Abs. 1 GenTG, $\S 40$ Abs. 1 Nr. 1 AMG, $\S \S 12$ Abs. 2, 17 Abs. 2, 19 Abs. 3 Nr. 2 ChemG, Nr. 1.5 Anl. 2 UVPG und $\S 36$ Abs. 2 S. 1 GefStoffV 54 auf das Risiko rekurriert, greift es auf den Risikobegriff erster Ordnung zurück. ${ }^{55}$ Dieser

\footnotetext{
${ }^{50} \mathrm{Vgl}$. WBGU (Fn. 28), 6, 285 ff.; ferner Heidenescher (Fn. 30), 230; Rehbinder in: Arbeitskreis für Umweltrecht (Hrsg.) Grundzüge des Umweltrechts, 2. Aufl. 1997, 04/50; Steinberg (Fn. 13), 149; Mrasek-Robor Technisches Risiko und Gewaltenteilung, 2000, 33 f.; Stoll Sicherheit als Aufgabe von Staat und Gesellschaft, 2003, 270.

51 Dazu unten IV.3.b).

52 Dabei lässt sich anknüpfen an Eigenschaften wie die Langlebigkeit, die Akkumulierbarkeit und die Mobilität eines Stoffes oder Produkts, an den Umfang der Stoffproduktion oder das Ausmaß der Exposition geschützter Güter. Zur Berücksichtigung dieser Faktoren im geltenden Recht Köck (Fn. 39), 56f.; eine weitergehende Einführung von Risikoindikatoren wird von verschiedenen Beratungsgremien empfohlen, vgl. WBGU (Fn. 28), 306ff.; European Environment Agency (Fn. 22), 170f.; Risikokommission „Neuordnung der Verfahren und Strukturen zur Risikobewertung und Standardsetzung im gesundheitlichen Umweltschutz der Bundesrepublik Deutschland“ (Hrsg.) Abschlussbericht 2003, 32ff., www.bmu.de/de/1024/js/download/b_rk/liste.php; s. auch Mathes/Ranke in: Hansjürgens (Hrsg.) Umweltrisikopolitik, ZAU-Sonderheft 10 (1999), 97, $101 \mathrm{ff}$.; kritisch allerdings Der Rat von Sachverständigen für Umweltfragen (Hrsg.) Sondergutachten Umwelt und Gesundheit, 1999, Tz. 29*, 137; allgemein zur Nutzung von Heuristiken als Mittel zur Sicherung von Entscheidungsrationalität unter Ungewissheit Gigerenzer (Fn. 14), 182.

53 Ein - wohl nicht in vollem Umfange überzeugendes - Beispiel bietet der Vorschlag der EG-Komission zur neuen Chemikalienregulierung: Weißbuch der Kommission der Europäischen Gemeinschaften "Strategie für eine zukünftige Chemikalienpolitik", KOM (2001), 88 endgültig, 13, 23 mit seiner Anknüpfung an die Produktionsmenge; kritisch dazu C. Calliess UTR 68 (2003), 11, 48ff.; vgl. auch Scheringer/Mathes/Weidemann/Winter ZAU 11 (1998), $227 \mathrm{ff}$.

54 Umfassende Aufzählung bei Kahl DVBl. 2003, 1105, 1107 (in Fn. 21).

55 Ausführlich zur Verwendung des Risikobegriffs im Verwaltungsrecht Karthaus (Fn. 39), 54 ff.; zum Gemeinschaftsrecht aus jüngster Zeit EuG Rs. T-70/99 v. 11. 9. 2002
} 
umfasst auch den Sonderfall der polizeirechtlichen Gefahr und lässt sich unabhängig davon verwenden, ob die konkreten Schutzanforderungen in Gefahrenabwehr, Risikovorsorge und Restrisiko abgestuft sind oder das Recht lediglich hinzunehmende und nicht hinzunehmende Risiken unterscheidet. ${ }^{56}$ Für den Umgang mit der Ungewissheit bietet der Risikobegriff erster Ordnung indes nur zirkuläre Gründe..$^{57}$ Die Verarbeitung der Irrtumskosten wird damit auf die Rechtsfolgenbestimmung verlagert.

So verlangt die Europäische Kommission bei der Anwendung des Vorsorgeprinzips in Anknüpfung an Art. 174 Abs. 3, 3. Spiegelstrich EGV eine Abwägung der positiven und negativen Auswirkungen der in Betracht zu ziehenden Maßnahmen und damit auch einen Vergleich der zu bearbeitenden mit den durch den Eingriff neu erzeugten Risiken. ${ }^{58}$ Den rechtlichen Rahmen für diesen Vergleich bieten die Abwägungs- ${ }^{59} \mathrm{Er}$ messens- ${ }^{60}$ und Vertretbarkeitsklauseln des Risikoverwaltungsrechts, ${ }^{61}$ die den Entscheidungsträgern einen Spielraum auch für die Frage zuweisen, wie die verbleibende Erkenntnisunsicherheit zu verarbeiten ist. ${ }^{62}$

(Alpharma/Rat), Slg. 2002, II-3495 Leitsatz 6 sowie Rn. 160, dort auch zu dem - gegenüber dem deutschen Recht - weiten gemeinschaftsrechtlichen Gefahrenbegriff.

56 Karthaus (Fn. 39), 69f.; Wahl ZLR 1998, 275, 282ff.; Köck (Fn. 39), 51 ff.

${ }^{57}$ So soll der zur Vorsorge berechtigende „Gefährlichkeitsverdacht" nach herrschendem Verständnis bei hohen, potentiell irreversiblen Schäden bereits bei schwacher Prognosegrundlage zu bejahen sein, obwohl das Urteil der Irreversibilität gerade auf dieser Prognoseschwäche beruht; dazu Rehbinder (Fn. 50), 04/50; Wahl/Appel (Fn. 23), 111f.; Kloepfer in: Gethmann/Kloepfer, Handeln unter Risiko im Umweltstaat, 1993, 55, 60; Köck in: Gawel (Hrsg.) Effizienz im Umweltrecht, 2001, 271, 281; zweifelhaft auch die Argumentation von Schulze-Fielitz (Fn. 3), 461 f., der aus der Ungewissheit über das Schadenspotential auf das Fehlen eines Risikos schließt. Indes ist zwischen der Eintrittswahrscheinlichkeit einer Hypothese und ihrer Plausibilität zu unterscheiden, vgl. nur Kleihauer in: Hansjürgens (Fn. 52), 50, 60; auf die Schwierigkeiten der Abgrenzung von Gefahr und Risiko unter Ungewissheit verweist überdeutlich VGH BW VBIBW 2001, 436ff., der in seiner tragenden Begründung vom Vorliegen einer Gefahr, in seinen Hilfserwägungen vom Vorliegen eines Vorsorgetatbestandes ausgeht.

${ }^{58}$ Kommission (Fn. 16), 22f.; für ihre Regelungsvorhaben hat die Kommission jüngst überdies eine Initiative für eine allgemeine Folgenabschätzung vorgestellt, vgl. Mitteilung der Kommission über Folgenabschätzung, KOM (2002), 0276 endgültig.

${ }^{59}$ Vgl. etwa $§ 2$ Abs. $1, \S 19$ Abs. 3 BNatSchG, $\$ 11,7$ Abs. $3, \S 9$ Abs. 1 BWaldG; $\$ 12$ UVPG; zu den impliziten Abwägungsgeboten in unbestimmten Rechtsbegriffen Kloepfer Umweltrecht, 2. Aufl. 1998, § 4 Rd. 54; Steinberg in: Riedel (Fn. 46), 17, $42 \mathrm{f}$.

${ }^{60}$ S. etwa $§ 17$ Abs. 1 BImSchG, $\$$ 7f. WHG; $\$ 7$ Abs. 2 AtG.

${ }^{61} \mathrm{Vgl} . \S 15$ Abs. 1 Nr. 3e PflSchG, $\S 16$ Abs. 1 Nr. 3 GenTG, $\$ 25$ Abs. 2 Nr. 5 AMG;

${ }^{62}$ Zum Verständnis der Vertretbarkeitsklauseln auch unten IV.3.a) (dd); ferner BVerwGE 81, 12, 17 zu $§ 15$ a. F. PfISchG; Tünnesen-Harmes Risikobewertung im Gentechnikrecht, 2000, $123 \mathrm{ff}$;; Köck (Fn. 39), 193 ff.; Di Fabio Risikoentscheidungen im Rechtsstaat, 1994, 153 ff., 278f.; Ladeur (Fn. 20), 239; Winter Die Prüfung der Freisetzung von gentechnisch veränderten Organismen, UBA-Berichte 4/98, $18 \mathrm{ff}$., $110 \mathrm{ff}$. 
Dazu schreiben etwa die gemeinschaftsrechtlichen Grundsätze für die Bewertung und Zulassung von Pflanzenschutzmitteln ausdrücklich vor, mögliche Unsicherheitsfaktoren bei den im Verlauf der Bewertung erhaltenen Informationen einzukalkulieren, um die Gefahr, schädliche Auswirkungen nicht zu erkennen [...], so gering wie möglich zu halten".63

Auch wenn damit unberücksichtigt bleibt, dass auch die Überschätzung schädlicher Auswirkungen eines Pflanzenschutzmittels zu einer ex post nicht erforderlichen Rechtsgutsbeeinträchtigung führt, ist dem Risikoverwaltungsrecht damit doch der Weg von der erfahrungsbezogenen Gefahrenabwehr zu einer ungewissheitsorientierten Risikosteuerung gewiesen, die ihre eigenen Grenzen und Risiken reflektiert. ${ }^{64}$

\section{Reflexive Risikosteuerung: Theoretische Grundlagen und Wirkungsbezïge}

\section{Recht als Instrument der Risikosteuerung}

Wird das Recht reflexiv, wird die es begleitende Rechtswissenschaft zur Steuerungswissenschaft. ${ }^{65} \mathrm{Im}$ Steuerungsbegriff ersetzt sie die klassisch rechtsaktsbezogene durch eine wirkungsbezogene Betrachtung des Rechts. ${ }^{66}$ Damit wird erkennbar, dass das Recht Wirkungen erzeugt, die der Absicht seiner Setzung zuwiderlaufen, etwa wenn es die gesellschaftliche Dynamik verfehlt mit überholten tatbestandlichen Erwartungen oder wenn es im Anwendungsprozess auf konkurrierende ökonomische oder soziale Imperative stößt. ${ }^{67}$

63 B.1.4. Anhang IV RL 91/414/EWG v. 15. 7. 1991 über das Inverkehrbringen von Pflanzenschutzmitteln, eingefügt durch Änderungsrichtlinie 97/57/EWG v. 22. 9. 1997, ABI. L 265/87.

${ }^{64}$ Hierzu auch Banse in: ders. (Fn. 2), 37f.; Tittes in: Compes (Hrsg.) Technische Risiken in der Industriegesellschaft, 1986, 345, 359; Binswanger ZfU 2 (1990), 103, $113 \mathrm{f}$.; Scheringer/Mathes/Weidemann/Winter(Fn. 53), $227 \mathrm{ff}$.

${ }^{65}$ Dazu Schmidt-Aßmann (Fn. 49), 1/33; Schuppert in: Hoffmann-Riem/Schmidt-ABmann/Schuppert (Fn. 35), 65 ff.; Hoffinann-Riem ebd., 115, $121 \mathrm{ff}$.; ein Selbstverständnis der Verwaltungswissenschaft als Steuerungswissenschaft wirft freilich sogleich die Frage nach ihrer Kompatibilität mit anderen Steuerungswissenschaften auf, vgl. Gawel in: Bizer/Linscheidt/Truger, Staatshandeln im Umweltschutz, 2000, 89, $94 \mathrm{ff}$.; nach einer ausgearbeiteten Theorie rechtlicher Steuerung sucht man in der Rechtswissenschaft bislang vergeblich; Vorarbeiten bei König/Dose Instrumente und Formen staatlichen Handelns, 1993; weitere Überlegungen bei Hoffinann-Riem/Schneider in: dies., Rechtswissenschaftliche Innovationsforschung, 1998, $405 \mathrm{ff}$.

66 Er fragt nach der „faktischen Kraft des Normativen“ vgl. v. Wangenheim in: Bizer/ Führ/Hüttig, Responsive Regulierung, 2002, 81, 85.

67 G. -P. Calliess (Fn. 6), 77; Kaufmann in: Grimm (Fn. 47), 15, $30 \mathrm{f}$. 
Die für die staatliche Steuerung in Betracht kommenden Steuerungsmedien sind nicht unbegrenzt verfügbar. ${ }^{68}$ Das gilt nicht nur für das Recht, für Geld und für Information, ${ }^{69}$ sondern vor allem für den Einsatz staatlicher Macht. ${ }^{70}$ Zwangsgewalt ist nicht nur aus rechtsstaatlichen Gründen die ultima ratio politischer Sozialgestaltung. ${ }^{71}$ Gerade im Umgang mit dem Risiko sind ihr auch funktionale Grenzen gesetzt. Solange etwa in der Frühphase einer technologischen Entwicklung noch verlässliche Maßstäbe für deren rechtliche Bewertung fehlen ${ }^{72}$ oder wenn es beim Umgang mit natürlichen Ressourcen um eine generelle Umsteuerung gesellschaftlicher Interaktionsmuster geht, ${ }^{73}$ ist der Staat auf die Wissensressourcen und Kooperationsbereitschaft der gesellschaftlichen Akteure angewiesen. ${ }^{74}$

Deshalb kann rechtliche Risikosteuerung nicht nach dem Modell der „vis absoluta“ als einseitige, womöglich deterministische Fremdbestimmung eines Steuerungsobjekts konzipiert werden, sondern ist auf Kommunikation umzustellen. ${ }^{75}$ Sie ist nicht zwangsweise Durchsetzung einer eigenen, sondern kommunikative Einwirkung auf fremde Rationalität, ${ }^{76}$ die sich über die wechselseitige Abgabe und Verarbeitung von Informationen über Realbedingungen und Verhaltenserwartungen zwischen den am Steuerungsprozess Beteiligten vollzieht. ${ }^{77}$

\footnotetext{
${ }^{68}$ Zum Ressourcencharakter der Steuerungsmedien etwa König/Dose (Fn. 65), 519, $551 \mathrm{ff}$., 555 .

${ }^{69}$ Zur Information als Steuerungsinstrument Kirchgässer in: Grimm (Fn. 47), 453, $457 \mathrm{ff}$.

70 Grimm in: ders. (Hrsg.) Die Zukunft der Verfassung, 2. Aufl. 1994, 399, 420ff.

${ }^{71}$ Vgl. Franzius (Fn. 49), 70.

72 Dazu Steigleder Kontrollierte Selbstregulierung in der naturwissenschaftlich-technischen Forschung, 2002, $94 \mathrm{ff}$.

${ }^{73}$ In unserem Zusammenhang also etwa um die Öffnung technologischer und ökonomischer Innovationsprozesse für die Anliegen präventiver Politik.

${ }^{74}$ Dazu allgemein Stolleis Zeitschrift für neuere Rechtsgeschichte 1989, $129 \mathrm{ff}$.; Treiber in: Dose/Voigt (Hrsg.) Kooperatives Recht, 1995, 65ff.

75 Dazu schon König Die Verwaltung 7 (1974), 137f.; ferner etwa v. Beyme Der Gesetzgeber, 1997, 19ff.; dazu auch Trute in: Hoffmann-Riem/Schneider (Fn. 65), 208, 243f.

${ }^{76}$ Halfmann in: Schulte (Fn. 3), 61, 63; Schulte in: Oebbecke/Bauer/Faber (Hrsg.) Umweltrecht und Kommunalrecht, 1998, 86, 95f.; am Beispiel der Einwirkung auf die Technikentwicklung Ekardt/Manger/Neuser/Pottschmidt/Roßnagel/Rust Rechtliche Risikosteuerung, 2000, $15 \mathrm{ff}$., $68 \mathrm{ff}$.; Kommunikation und Kooperation spielten schon in der Praxis der Verwaltung des 19. und frühen 20. Jahrhunderts eine stärkere Rolle als dies die Staatsrechtslehre seinerzeit reflektierte; dazu Ellwein in: Dose/Voigt (Fn. 74), 43ff.; Franzius (Fn. 49), $230 \mathrm{ff}$.

77 So im Ansatz etwa Schmidt-AßMmann (Fn. 49), 1/36.
} 
Bei der Analyse der Erfolgsbedingungen dieses Prozesses kann sich die Rechtswissenschaft auf die Erkenntnisse der Verhaltenswissenschaften stützen. ${ }^{78}$ Danach orientieren sich Individuen und Organisationen nicht allein an ökonomischen Rationalitäten, sondern auch an Identitäten, Leitbildern und Visionen. ${ }^{79}$ Diese verkörpern kulturelle, organisationelle und familiäre Regeln und Heuristiken, die sich aus der Zugehörigkeit zu einer Gruppe und der Identifikation mit ihr erklären. ${ }^{80}$ Neben die Nutzenmaximierung tritt damit die Regelorientierung als eine auf die Wahrung von Gruppeninteressen bezogene Form menschlicher Entscheidungsrationalität. ${ }^{81}$ Entgegen der Annahmen des klassischen rational-choice Modells ${ }^{82}$ ist diese Präferenzstruktur nicht vorfindlich und konstant, sondern entwickelt sich anhand der Bedingungen der konkreten Entscheidungssituation fort. ${ }^{83}$ Sie steht deshalb gerade auch rechtlicher Einwirkung offen. ${ }^{84}$

Allerdings konkurriert das Recht dabei mit anderen gesellschaftlichen Koordinationsformen. ${ }^{85}$ Rechtliche Risikosteuerung muss deshalb die regelmäßige Abhängigkeit der Steuerungsadressaten von sozialer Akzeptanz, wirtschaftlichem Erfolg und langfristigen Planungshorizonten und

78 Dazu Bizer/Führ in: dies./Hüttig (Fn. 66), 1, 7.

79 Nonaka Harvard Business Review 1991, $96 \mathrm{ff}$; Simon Journal of Economic Perspectives 5 (1991), 25, 29ff.; March/Olson Ambiguity and Choice in Organisations, 1976, 26ff., 36f., $71 \mathrm{ff}$; dies. Rediscovering Institutions, 1989, $159 \mathrm{ff}$.; Brentel Soziale Rationalität, 1999, 237 ff.; vgl. auch schon March/Simon Organisation und Individuum, 1976, $37 \mathrm{ff}$., $81 \mathrm{ff}$., $129 \mathrm{ff}$.; Mayntz/Scharpfin: dies. (Hrsg.) Gesellschaftliche Selbstregelung und politische Steuerung, 1995, 9 ff., 52ff.; Dierkes Zeitschrift für Betriebswirtschaft 58 (1988), $554 \mathrm{ff}$.

${ }^{80}$ Dazu bereits Scherzberg Wozu und wie eigentlich noch öffentliches Recht? 2003, $28 \mathrm{ff}$.

${ }^{81}$ March A Primer on Decision Making, 1994, 57ff.; vgl. auch Etzioni The moral dimension, 1988, $185 \mathrm{ff}$.

82 Dazu etwa Hargreaves/Hollis/Weale The Theory of Choice, 1992, $3 \mathrm{ff}$. und passim; Becker Der ökonomische Ansatz zur Erklärung menschlichen Verhaltens, 1993, $3 \mathrm{ff}$;; Mueller Public choice II, 1989, 43 ff., $424 \mathrm{ff}$.; ferner Braun Theorien rationalen Handelns in der Politikwissenschaft, 1999, $22 \mathrm{ff}$. und die Beiträge in Nida-Rümelin (Hrsg.) Praktische Rationalität, 1994; dazu aus Sicht der Rechtswissenschaft Bizer/Führ und Oeter in: Bizer/Führ/Hüttig (Fn. 66), 1 ff., 195ff.; Kirchnerin: Hoffmann-Riem/Schneider (Fn. 65), $85,92 \mathrm{f}$. sowie jüngst $v$. Aaken „Rational Choice“ in der Rechtswissenschaft, 2003, $64 \mathrm{ff}$. und passim.

83 Priddat in: Banse (Fn. 2), $119 \mathrm{ff}$; Mayntz/Scharpf(Fn. 79), $52 \mathrm{ff} . ;$ Messner Die Netzwerkgesellschaft, 1995, 176 ff.; Scherzberg(Fn. 80), 29f.; J. Hofmann Implizite Theorien in der Politik, 1993, $211 \mathrm{ff}$.

${ }^{44}$ Dazu etwa Engel in: Rengeling (Hrsg.) Instrumente des Umweltschutzes im Wirkungsverbund, 2001, 17, 31, $33 \mathrm{f}$.

85 Kitschelt in: Grimm (Fn. 47), 391, 417. 
auch die Kontextbedingungen des konkreten Steuerungsprozesses in Rechnung stellen. ${ }^{86}$ Erfolgreiche Risikosteuerung ist demnach ,responsive Regulierung“.87

\section{Risiko und Innovation als Gegenstand rechtlicher Steuerung \\ a) Die Konstituierung des Risikos}

Risikosteuerung beginnt mit der Risikokonstitution. ${ }^{88}$ Das Recht definiert schutzwürdige Güter, selektiert Entwicklungspfade, die es als unerwünscht bewertet, und rechnet erwartete Folgen einer angenommenen Ausgangsbedingung als verantwortlich zu. ${ }^{89}$ Freilich kann es Zurechnungen außerhalb des Bereichs alltagsweltlicher Erfahrung nicht ohne Rückgriff auf die Ergebnisse der Risikoforschung begründen..$^{90}$

Diese macht dazu allerdings Aussagen von höchst unterschiedlicher Validität. ${ }^{91}$ Erhebliche Erkenntnisunsicherheiten gibt es vor allem in der Ökotoxikologie und in der Epidemiologie. ${ }^{92}$ Ihnen fehlen Kriterien und Methoden zur sicheren Extrapolation von Labor- auf Realbedingungen,

${ }^{86}$ Dazu Messner(Fn. 83), XI, 138, 142; Hoffmann-Riem (Fn. 47), 34; Steinberg(Fn. 13), 396f.; Braczyk in: Rammert/Bechmann (Hrsg.) Technik und Gesellschaft, Jahrbuch 9 (1997), $251 \mathrm{ff}$;; mit Auswertung entsprechender Fallstudien Ekardt/Manger/Neuser/Pottschmidt/Roßnagel/Rust (Fn. 76), $143 \mathrm{ff}$.

${ }^{87}$ Dazu Ayres/Braithwaite Responsive Regulation, 1992; Bizer/Führ/Hüttig (Fn. 66); Tidelski in: Bizer/Linscheidt/Truger (Fn. 65), 111, 126f.; vgl. auch Bora in: ders. (Fn. 26), $9,17 \mathrm{ff}$.

${ }^{88}$ Zur Risikokonstitution allgemein Bryde in: Grabes (Hrsg.) Wissenschaft und neues Weltbild, 1992, 71ff., 80; am Beispiel des GenTG Winter in: Umweltbundesamt (Hrsg.) Die Abwägung von Risiken und Kosten in der europäischen Chemikalienregulierung, $1999,111 \mathrm{ff}$.

${ }^{89}$ Dazu Köck in: Bora (Fn. 26), $148 \mathrm{ff}$.

90 BVerwGE 72, 300, 316.

91 Ausführlich dazu Neus in: Umweltbundesamt (Hrsg.) Aktionsprogramm Umwelt und Gesundheit, 1998, $47 \mathrm{ff}$; die Abschätzungsunsicherheit setzt sich in einer unterschiedlichen Beurteilung der Zuverlässigkeit von Risikoabschätzungen fort, vgl. einerseits Sachverständigenrat, Sondergutachten (Fn. 52), Tz. 29*, 137; andererseits Neus (Fn. 91), passim. Dabei stoßen nicht selten „Schulwissenschaft“ und „kritische Wissenschaft" aufeinander, dazu Sachverständigenrat ebd., Tz. 117. Der Sachverständigenrat hält es für eine politische Entscheidung, welcher „Richtung“ zu folgen sei, ebd., Tz. 118.

92 Zur Epidemiologie vgl. Neus (Fn. 91), 74f.; Sachverständigenrat, Sondergutachten (Fn. 52), Tz. 8* ff., $97 \mathrm{ff}$;; Drexler/Diepgen Zbl Arbeitsmed 50 (2000), 374ff.; Banse in: ders. (Fn. 2), 15, 64 f.; Stevens/Foerster MED SACH 99 (2003), 104, 105f.; exemplarisch auch Hauptverband der gewerblichen Berufsgenossenschaften (Hrsg.) Risikofaktoren für Plattenepithelkarzinome im Kopf-Hals-Bereich, 1994, $124 \mathrm{ff}$;; zu vergleichbaren Problemen bei der Beurteilung der ökologischen Folgen einer Freisetzung genetisch modifizierter Organismen v. Schomberg Der rationale Umgang mit Unsicherheit, 1995, $103 \mathrm{ff}$. 
vom Tierversuch auf den Menschen, zur Wirkungsanalyse von Stoffgemischen und zur Festlegung von Sicherheitszuschlägen für Personen mit besonderer Empfindlichkeit. ${ }^{93}$ Bei Kanzerogenen weichen wissenschaftlich vertretbare Schätzwerte durchschnittlich um einen Faktor von 100 voneinander ab..$^{94}$ Dass die Schätzergebnisse der Experten nicht nur in der "Sach-“ sondern auch in der „Sozialdimension" des Risikos ${ }^{95}$ wurzeln, zeigen Studien an Mitgliedern der kanadischen und der US-amerikanischen Gesellschaft für Toxikologie. Danach wird das Expertenurteil von weltanschaulichen Überzeugungen und selbst vom Geschlecht der Befragten beeinflusst und hängt - ohne jede persönliche Beteiligung auch davon ab, ob die Experten sich einem Problem aus der Perspektive der Industrie, der Verwaltung oder der Wissenschaft nähern. ${ }^{96}$

Grundlegend für die Konstitution des Risikos werden deshalb die Standardannahmen und Konventionen zur Überbrückung der Abschätzungsunsicherheit. ${ }^{97}$ Gerade die hierzu entwickelten Regeln überschreiten aber den Bereich des Empirisch-Kognitiven und sind nicht selten von wissenschaftlich nicht belegten Denkmustern und Werthaltungen geprägt. ${ }^{98}$

93 Neus (Fn. 91), 68f.; Cranor Regulating Toxic Substances, 1993, 15ff. Ungewiss ist ferner, ob die niedrigste Dosis, bei der adverse Reaktionen beobachtet werden, die höchste Dosis, bei der diese Wirkungen nicht auftreten, oder eine Benchmark, bei der nachteilige Reaktionen mit einer bestimmten Wahrscheinlichkeit eintreten, als Ausgangspunkt für die Risikodefinition genommen werden sollen, vgl. Neus (Fn. 91), 111f.; dazu auch Anhang I der RL 93/67/EWG zur Festlegung von Grundsätzen für die Bewertung der Risiken für Mensch und Umwelt von gemäß der RL 67/548/EWG notifizierten Stoffen, ABl. L 227/9ff.

94 Cranor (Fn. 93), Appendix A; s. auch Sexton/Reiler/Zenick Toxicology 102 (1995), $3 \mathrm{ff}$.

95 Dazu Japp (Fn. 20), 19 f.

${ }^{96}$ Kraus/Malmfors/Slovic Risk Analysis 12, 1992, $215 \mathrm{ff}$.; soweit bei den Schätzergebnissen von Expertengremien höhere Konvergenzen auftreten, sind diese häufig auf vereinfachende Annahmen zurückzuführen, bei denen Wechselwirkungen und nichtlineare Effekte vernachlässigt werden, vgl. Neus (Fn. 91), 79f.; Teuschler/Hertzberg Toxicology 105 (1995), $137 \mathrm{ff}$.

${ }^{77}$ Sie betreffen neben den oben genannten Extrapolationen und Sicherheitsannahmen auch den Umgang mit divergierenden Befunden und die Abwägung von Kosten und Nutzen weiterer Risikoermittlung; dazu Neus (Fn. 91), 83, 89 ff., 109ff.; Ladeur (Fn. 20), $127 \mathrm{ff}$.

98 Risikokommission Neuordnung der Verfahren und Strukturen zur Risikobewertung und Standardsetzung im gesundheitlichen Umweltschutz der Bundesrepublik Deutschland - Erster Bericht, 2002, 58; Neus (Fn. 91), 66; Ueberhorst/de Man in: Schütz (Hrsg.) Risiko und Wagnis, 1990 Bd. I, 81 ff.; Weinberg Minerva 10 (1972), 209 ff.; dazu auch Ladeur (Fn. 20), 87 ff.; Kleihauer in: Hansjürgens (Fn. 52), 50, 52ff.; Cansier/Cansier in: Hansjürgens (Fn. 52), 157, 158f.; instruktiv insoweit der Streit um die Leukämiefälle in der Umgebung des Kernkraftwerkes Krümmel, dazu Wiedemann/Mertens/Clauberg/ 
Angesichts dieses Befundes darf das Recht die Konstitution des Risikos nicht einfach an die Risikoforschung delegieren, ${ }^{99}$ sondern muss die naturwissenschaftliche Realanalyse mit ihren Konventionen an rechtlich spezifizierte Schutzziele zurückbinden und den Umgang mit Abschätzungsunsicherheiten im Übrigen standardisieren. ${ }^{100}$ Empfehlungen hierzu hat kürzlich die „Risikokommission“ zweier Bundesministerien vorgelegt. ${ }^{101}$

\section{b) Risikobewertung und Risikovergleich}

Im Rahmen der Risikobewertung entscheidet das Recht über die rechtliche Erheblichkeit und Akzeptabilität des Risikos. ${ }^{102}$ Auch hierfür gibt es kein allgemeingültiges Maß. ${ }^{103}$ So lassen sich Risiken nur vor dem Hintergrund der den Betroffenen im Schadensfall verbleibenden Ressourcen bewerten ${ }^{104}$ und fließen darin auch die von der psychologischen Risikoforschung aufgedeckten individuellen und kollektiven Muster der Risikoverarbeitung ein. ${ }^{105}$

Hennings/Schütz Umweltstandards, Abschlussbericht BfS-Projekt StSch 4337, 2002, $48 \mathrm{ff}$., www.bmu.de/de/1024/js/download/b_rk/liste.php; Experten verneinten eine Ursächlichkeit von Emissionen des Kraftwerks unter Berufung auf die Einhaltung der geltenden Grenzwerte und der dabei unterstellten linearen Dosis-Wirkungs-Beziehung. Neuste Forschungen deuten aber darauf hin, dass geringe Strahlungsdosen ganz andere Kausalketten in Gang setzen als hohe Dosen, was die Konvention der linearen Verknüpfung von Dosis und Wirkung möglicherweise in Frage stellt; dazu Focus 17/2003, 114.

99 Dazu Schulze-Fielitz (Fn. 3), 449f.; Kastendieck Der Begriff der praktischen Vernunft in der juristischen Argumentation, 2000, 88f.; Breuer Gutachten B zum 59. DJT, 1992, B $64 \mathrm{f}$.

${ }^{100}$ Risikokommission, Abschlussbericht (Fn. 52), 13 f.; ferner Winter ZUR 1994, 20, $22 \mathrm{ff}$.; National Research Council (Hrsg.) Science and Judgement, 1994, 25 ff., $80 \mathrm{ff}$.; dazu Neus (Fn. 91), 9f.; vgl. auch Grunwald in: Kloepfer (Fn. 48), 43, 53 ff.

101 Vgl. Risikokommission, Abschlussbericht (Fn. 52), 71 ff., der Abschlussbericht enthält u.a. den Entwurf eines Gesetzes „für Standardsetzung zum Schutz der menschlichen Gesundheit und der Umwelt" und einen Leitfaden zur Risikoabschätzung. Regeln zur Verarbeitung von Abschätzungsungewissheit bietet auch Anhang I RL 93/67/EWG und das dazu ergangene Technical Guidance Document; vgl. auch Kommission (Fn. 16), $15 f$.

102 S. etwa Sachverständigenrat, Sondergutachten (Fn. 52), Tz. 27*, 130.

103 Bechmann (Fn. 7), 228; Helten in: Gesamtverband der deutschen Versicherungswirtschaft e.V. (Fn. 1), 193, 204; etwa könnte es angesichts des Katastrophenpotentials moderner Technik und der Ungewissheit bei der Bestimmung des Wahrscheinlichkeitskoeffizienten vernünftig erscheinen, den Schadensaspekt höher und die Wahrscheinlichkeit geringer zu gewichten, vgl. Wiedemann in: Bayerische Rück (Fn. 8), 43, 60

104 Stoll (Fn. 50), 169.

105 Dazu Holzheu (Fn. 8), 267; Hapke/Japp Prävention und Umwelthaftung, 2001, 76ff.; Krelle Präferenz- und Entscheidungstheorie, 1968, 147; zu kulturellen Mustern der Verarbeitung von Risiken vgl. Dressel BSE - The New Dimension of Uncertainty, 2002, $33 \mathrm{ff}$. 
Danach bestimmen die Freiwilligkeit, die Vertrautheit, die Zurechenbarkeit, die Kontrollierbarkeit, die Zeitnähe und die Streuung die Wahrnehmung des Risikos ${ }^{106}$ und wiegen die Chancen eines Gewinnes geringer als der äquivalente Erwartungswert eines Verlustes. ${ }^{107}$ Ein Gesundheitsrisiko kann also unterschiedlich zu bewerten sein, je nachdem ob der Schaden als Folge einer Immission, der Teilnahme am Straßenverkehr oder der Einnahme eines Arzneimittels erwartet wird.108 Quantitative, gar monetarisierte Kosten-Nutzen-Analysen, wie sie in den USA zur Bewertung von Risiken und zur Festlegung von Umweltstandards vorgesehen sind, ${ }^{109}$ verschleiern die insoweit zu treffenden Wertungen und verschieben sie, worauf jüngst Dietrich Murswiek hingewiesen hat, auf die dann anschließend doch qualitativ zu beantwortende Frage, ob die erwartete Minderung des Risikos die Kosten der Maßnahme trägt. 110

Für diese Abwägung bedarf es eines Risikovergleichs. ${ }^{111}$ Dieser verkörpert die Rationalität der Risikosteuerung und in seiner Komplexität auch ihre Grenzen. ${ }^{112}$ Er zielt auf eine Bewertung der Folgen unterschied-

106 Jungermann/Slovic in: Bechmann (Hrsg.) Risiko und Gesellschaft, 2. Aufl. 1997, 167, 173, 193 ff.; Jungermann/Pfister/Fischer Die Psychologie der Entscheidung, 1998, 195ff., 210ff.; Bechmann (Fn. 7), 232; Gill/Bizer/Roller (Fn. 40), 298.

107 Jungermann/Pfister/Fischer(Fn. 106), $223 \mathrm{f}$.

108 v. Lersner in: Gawel/Lübbe-Wolff (Hrsg.) Rationale Umweltpolitik - rationales Umweltrecht, 1999, 227, 228f.; zur Relativierung des Sicherheitszwecks auch Hoffimann-Riem ZRP 2002, 497, 501.

${ }^{109}$ Etwa in $\$ 1412$ (b)(3)(c) Safe Drinking Water Act (SDWA), wo eine eingehende Kosten-Nutzen-Analyse Voraussetzung zur Setzung von „maximum containment levels“ ist; $\S 111$ (a)(1) Clean Air Act (CAA), wonach bei der Setzung von ,standards of performance" zur Emissionsbegrenzung die Kosten der Emissionsreduzierung zu berücksichtigen sind; $\S 312$ CAA, welcher die EPA seit 1990 zu einer regelmäßigen Kosten-NutzenAnalyse in Bezug auf die gesamte Luftreinhaltepolitik nach dem CAA verpflichtet. Weitere Bsp. in $\S 6$ (a) Toxic Substances Control Act (TSCA), $\S 3$ (c)(5), 6(b), 2 (z)(bb) Federal Insecticide, Fungicide and Rodenticide Act (FIFRA); befürwortend Sunstein (Fn. 44), 191 ff.; ders. Chicago Journal of Legal Studies 29 (2002), 1059 ff.; kritisch McGarity Administrative Law Review 50 (1998), $7 \mathrm{ff}$.

${ }^{110}$ Murswick in: Jahrbuch Umwelt- und Technikrecht 71, 2003 (im Erscheinen), MS, 31 ff., 36; Buchholz Integrative Grenzwerte im Umweltrecht, 2001, 44; zu Kosten und Nutzen von Kosten-Nutzen-Analysen ausführlich Köck (Fn. 57), 290ff.; Hansjürgens in Gawel (Fn. 57), 63 ff.; Schütz in: Schütz/Wiedemann, Technik kontrovers, 1993, $108 \mathrm{ff}$; ausführlich zur mittelbaren Monetarisierung Nowitzki (Fn. 42), $131 \mathrm{ff}$; f ferner Voßkuhle Das Kompensationsprinzip, 1999, $398 \mathrm{ff}$.

111 Dazu Di Fabio (Fn. 15), 824ff.; Köck (Fn. 57), 293f.; ausführlich zu den verschiedenen Modellen des Risikovergleichs Seiler Recht und technische Risiken, 1997, $223 \mathrm{ff}$.

112 Dazu Stoll (Fn. 50), 340f.; Köck in: Bora (Fn. 26), 156f.; G.-P. Calliess (Fn. 6), 79 f. 
licher Maßnahmen der Risikoreduktion ${ }^{113}$ und bezieht darin den meist sicheren Nutzen eines Stoffes, seine regelmäßig ungewissen Nebenfolgen, ${ }^{114}$ eine entsprechende Bilanz für Substitute und alternative Entwicklungspfade, ${ }^{115}$ die soziale Verteilung der Risiken, die Folgen einer Fehlsteuerung und das Ausmaß der bei der Beurteilung bestehenden Ungewissheit ein. ${ }^{116}$ In den Blick zu nehmen sind dabei nicht zuletzt die Auswirkungen der Maßnahmen auf eine das Risiko begründende Innovation.

\section{c) Die Bewertung von Innovationen}

Unter Innovation wird in Anlehnung an die wirtschaftswissenschaftliche Begriffsbestimmung die Entwicklung und Einführung neuer technischer Verfahren, Anwendungen oder Produkte verstanden, die typischerweise mit dem Ziel verbunden ist, sie wirtschaftlich mit Gewinn zu verwerten. ${ }^{117}$ Folgt man Ikujiro Nonaka bei seiner Analyse des Erfolgs der japanischen Automobilindustrie, entstehen Innovationen, wenn Metaphern und Leitbilder dazu motivieren, explizites und implizites Wissen bei der Suche nach neuen Potentialen zu kombinieren. ${ }^{118}$ Nur auf diese Weise lässt sich, wie die Kybernetik zeigt, die Steigerung der Umweltkomplexität eines Systems angemessen verarbeiten ${ }^{119}$ und damit seine Zukunftsfähigkeit sichern. ${ }^{120}$

${ }^{113}$ Köck in: Hansjürgens (Fn. 52), 76, 85 (dort insb. Fn. 66).

114 Ggf. unter Vergleich mit dem Maß der natürlichen Exposition, s. dazu BVerwGE 61, 256, 265; dieser Vergleich muss die Entscheidung aber schon deshalb nicht prägen, weil sich durch das künstlich geschaffene Risiko die Risikogesamtlast erhöht.

115 Ggf. unter Einschluss sog. risk-tradeoffs, dazu Wiener/Graham in: dies. (Fn. 21), $1 \mathrm{ff} ., 226 \mathrm{ff}$. In den USA kommt das Verbot eines Stoffes, der gänzlich neue Wirkungsfelder erschließt, danach praktisch nur bei Existenz eines weniger gefährlichen Ersatzstoffes in Betracht; s. US Court of Appeals, 5th Circuit, 947 F.2d 1201, $1220 \mathrm{ff}$. (Corrision Proof Fittings); zu Defiziten der Alternativenprüfung bei der geplanten EG-Chemikalienregulierung C. Calliess (Fn. 53), $57 \mathrm{ff}$; vgl. dazu die Verordnungsermächtigung des $\S 17$ Abs. 2 ChemG.

116 Dazu etwa VGHBW VBl 2002, 436, $437 \mathrm{ff}$., der Ungewissheit durch einen „Sicherheitszuschlag“ zu Grenzwerten eines bekannten Stoffes verarbeitet; allgemein Seiler (Fn. 111), 232ff.; Mathes/Ranke in: Hansjürgens (Fn. 52), 97, 103; Scherzberg (Fn. 47), $132 \mathrm{f}$.

117 Freeman in: Eatwell/Milgate/Newman (Hrsg.) The New Palgrave - A dictionary of economics II, 1992, 858; Grupp/Schmoch in: Halfmann/Bechmann/Rammert (Hrsg.) Technik und Gesellschaft Jahrbuch 8 (1995), $227 \mathrm{ff}$; s. auch Hauschildt in: HoffmannRiem/Schneider (Fn. 65), 29 ff.; J. Hofimann (Fn. 83), 36ff., 52.

118 Nonaka (Fn. 79), 99 ff.

119 Ashby Einführung in die Kybernetik, 1974, $293 \mathrm{ff}$.

120 WBGU (Fn. 28), 288. 
Sind Innovationen mit Risiken verbunden, bedürfen sie einer Bewertung. Eine Regel hierfür bietet Hans Jonas. Aus dem „Prinzip Verantwortung“ folgt für ihn die Verpflichtung, bei neuen Risiken die schlechteste Prognose zugrundezulegen und deren Realisierung zu vermeiden. ${ }^{121} \mathrm{Al}-$ lerdings führt diese Strategie nicht in einen ,idyllischen Naturzustand“ zurück, ${ }^{122}$ sondern zu einer älteren Technik, zu deren bekannten Risiken sich, man denke an die FCKW, durch Synergismen, Akkumulationen und zeitliche und räumliche Fernwirkungen durchaus noch neue Schadenspotentiale gesellen können. ${ }^{123}$

Im Zweifel zugunsten der Innovation streitet dagegen Aaron Wildavsky, der Risikosteuerung primär als Hemmnis des Erwerbs risikorelevanten Wissens betrachtet und darauf verweist, dass eine marktwirtschaftliche Ordnung auf die Chance zum Lernen aus Irrtümern und Fehlern angewiesen sei.124 Erfahrung ist indes nur für den Überlebenden ein $\mathrm{Ge}-$ winn. $^{125}$ Angesichts der hohen Entwicklungsrisiken moderner Technik kann deren Einführung nur als kontrolliertes Experiment zulässig sein. ${ }^{126}$ Das Vertrauen auf Lerneffekte ist, wie zuletzt das britische BSE-Missmanagement zeigt, ${ }^{127}$ als Methode der Risikobearbeitung unvertretbar, wenn gravierende Folgen drohen.

Die Zukunftsfähigkeit der Gesellschaft kann durch die Verwirklichung von Risiken ebenso beeinträchtigt werden wie durch das Unterlassen der Nutzung von Chancen. Deshalb darf staatliche Risikosteuerung weder Ungewissheit verdrängen noch Erfahrungsgewinn vermeiden, ${ }^{128}$ sondern

121 Jonas Das Prinzip Verantwortung, 1979, 70ff., $391 \mathrm{ff}$; zustimmend Wolf KritV 80 (1997), 280, $295 \mathrm{f}$.

122 v. d. Daele in: Krohn/Krücken (Fn. 30), 284, 298.

123 Irreversibilitäten lassen sich eben nur um den Preis anderer Irreversibilitäten vermeiden; vgl. Banse in: ders. (Fn. 2), 33f.; Kerwer in: Hiller/Krücken (Fn. 26), 253, 264 ff.; v. d. Daele in: Krohn/Krücken (Fn. 30), 284, 300f.; Mrasek-Robor (Fn. 50), 160; kritisch zur „Heuristik der Furcht" deshalb etwa auch Spiecker gen. Döhmann (Fn. 20), 81.

124 Wildavky Searching for Safety, 1988, $17 \mathrm{ff}$; ders. in: Krohn/Krücken (Fn. 30), $305 \mathrm{ff}$.; dazu Kerwer in: Hiller/Krücken (Fn. 26), 253ff.; Gollier Economic Policy 2001, 303, 305; Holm/Harris Nature 400 (1999), 398; für eine versuchsweise Zulassung risikoträchtiger Technologien deshalb auch Wolf in: Bora, (Fn. 26), 65, 86.

125 Analysen im Bereich des Arbeitsschutzes deuten etwa darauf hin, dass sich bei neuen Technologien lediglich die Gefährdungsschwerpunkte von der Produktions- auf die Errichtungs-, Montage- und Probelaufphase verschieben, insgesamt die Risiken aber nicht mindern, näher Bücker Von der Gefahrenabwehr zu Risikovorsorge und Risikomanagement im Arbeitsschutzrecht, 1997, 70ff.; FinndorffDer Maschinenschaden, 1991, $49 \mathrm{ff}$.

126 Murswiek VVDStRL 48 (1990), 207, 216f.

127 Hierzu Dressel (Fn. 105), $39 \mathrm{ff} ., 49 \mathrm{ff}$.

${ }_{128}$ Bryde (Fn. 88), 81 f.; Wolfin: Bora (Fn. 26), 65, 85f.; Gill/Bizer/Roller (Fn. 40), 27. 
muss sich an dem Ziel orientieren, die gesellschaftliche Handlungsfähigkeit insgesamt zu erhöhen. ${ }^{129} \mathrm{Ob}$ und inwieweit bei Innovationen aber die Steigerung von Handlungsoptionen oder die Begründung von Risiken überwiegt, ist auf der Basis vorhandenen Wissens regelmäßig kaum zu beurteilen. ${ }^{130}$ Denn eine Innovation schafft neues Wissen, das vergangene Erfahrungen revidiert. ${ }^{131}$ Wenn sich staatliche Risikosteuerung also allein auf die Referenz des bereits Bekannten verließe, wäre eines mit Sicherheit riskant: die rechtliche Regulierung selbst.

\section{d) Risikosteuerung als innovativer Prozess}

Risikosteuerung ist deshalb so auszugestalten, dass sie neues Wissen generiert und sich Regulierung und technischer Innovationsprozess damit wechselseitig stärken. ${ }^{132}$ Das Recht markiert dabei die grundlegenden Sicherheitserwartungen der Gesellschaft und macht so negative ökologische Konsequenzen erst als solche erkennbar. Es definiert damit langfristige Bedingungen und Ziele, unter denen im Innovationsprozess gelernt werden kann.

Deren Umsetzung gelingt freilich ohne retardierende Elemente nur, wenn sich das Recht im konkreten Regulierungsprozess des jeweiligen Standes von Wissenschaft und Technik versichert, dessen Weiterentwicklung fördert und seine Ergebnisse integriert. ${ }^{133}$ Dazu bedarf es eines funktionierenden informationellen Netzwerks von Rechtssetzung und Rechtsanwendung, Forschung, Technik und Industrie.134 Die Konzepte und Steuerungsimpulse der konkreten Risikoreduktion sind aus dieser Interaktion und damit aus einer kooperativen und prozeduralen Grundstruktur heraus zu entwickeln. ${ }^{135}$ Die Unsicherheit über das Ausmaß not-

\footnotetext{
129 Gemäss Willke Ironie des Staates, 1992, 287; Ladeur KritV 74 (1991), 241, 252 und v. Foerster Sicht und Einsicht, 1985, 41 gilt die Erweiterung von Handlungsoptionen sogar als ethischer Imperativ.

130 Groys in: Rammert/Bechmann (Fn. 86), 15ff.; Zimmermann/Pahl in: Hansjürgens (Fn. 52), $107 \mathrm{ff}$.

131 Dazu lesenswert Priddat in: Banse (Fn. 2), $108 \mathrm{f}$.

132 Kerwer in: Hiller/Krücken (Fn. 26), 253, $268 \mathrm{ff}$.

$133 \mathrm{Zu}$ Langfristorientierung und Flexibilität etwa Klemmer/Lehr/Löbbe Umweltinnovationen, 1999, 111; Grunwald Technik für die Gesellschaft von morgen, 2000, $24 \mathrm{f}$.

134 Ekardt/Manger/Neuser/Pottschmidt/Roßnagel/Rust (Fn. 76), 271 ff.; Sachverständigenrat (Fn. 22), Tz. 53; Grunwald (Fn. 133), 152f.; Benz in: Hoffmann-Riem/Schneider (Fn. 65), 121, 133 ff.; Pitschas in: Kloepfer (Hrsg.) Technikentwicklung und Technikrechtsentwicklung, 2000, 73, 90ff.; Beispiel bei Krohn UTR 43 (1998), 178ff.; allgemein auch Trute (Fn. 46), 103.

135 Kuhlmann/Bättig/Cuhls/Peter Regulation und künftige Technikentwicklung, 1998, $22 \mathrm{ff}$; ; also nicht „learn before you act“ oder „act before you (have to) learn“, sondern
} 
wendiger Prävention und das Risiko der Fehlsteuerung werden damit schrittweise gemindert. Das gelingt insbesondere durch eine Privilegierung von Versuchsanlagen, die Abstufung der Zulassungsanforderungen nach Maßgabe des Risikowissens und die Verknüpfung von Zulassung und Risikobegleitforschung. ${ }^{136}$ In dieser Wechselwirkung von Innovation und Intervention wird rechtliche Steuerung selbst innovativ. ${ }^{137}$

Sie kann damit technische Innovationen anregen und in eine gewünschte Richtung lenken, wenn der rechtlich erzeugte Druck in geeigneter Weise auf die Innovationsfähigkeit und die verfügbaren Ressourcen der Akteure abgestimmt ist. ${ }^{138}$ Das kann eine Förderung der gesellschaftlichen Innovations- und Informationsinfrastruktur voraussetzen, ${ }^{139}$ erfordert aber vor allem geeignete Instrumente der Innovationspolitik.

\section{e) Risikosteuerung als Steuerung von Innovationen}

Die Umwelt- und Innovationsökonomie schlägt hierfür einen Mix aus ökonomischen und ordnungsrechtlichen Elementen vor. ${ }^{140}$ Ökonomische Instrumente können vor allem durch Vermeidungsanreize zur flexiblen Risikominimierung motivieren. ${ }^{141}$ Hierzu tragen etwa Haftungsund (faktische) Versicherungspflichten bei, die auch das Risikowissen der Haftpflichtversicherer aktivieren, ${ }^{142}$ sowie deliktische Produktbeobach-

\footnotetext{
„learn by acting.“ Zum Ganzen auch Gollier (Fn. 124), 303 ff.; Pitschas in: Kloepfer (Fn. 134), 73, $81 \mathrm{ff}$.

${ }^{136}$ Vgl. $\S 2$ Abs. 3 der 4. BImSchV; $\S 6$ Abs. $2, \S 7$ Abs. 1 , $\$ 8,11$ Abs. $1, \S 16$ Abs. 1 GenTG; $\$ 28$ Abs. 3 a, 29 Abs. 1 AMG.

137 Benz in: Hoffmann-Riem/Schneider (Fn. 65), 121, 131.

138 Wegner (Fn. 19), 38; dazu etwa J.-P. Schneider in: Eifert/Hoffmann-Riem (Hrsg.) Innovation und rechtliche Regulierung, 2002, 264, $268 \mathrm{ff}$.

139 Ausführlich zu strukturellen Innovationshemmnissen und staatlichen Handlungsmöglichkeiten Umweltbundesamt (Hrsg.) Innovationspotentiale von Umwelttechnologien, 1998, $353 \mathrm{ff}$.; dazu auch die Stellungnahme des Sachverständigenrates (Fn. 22), Tz. 11*, 42 ff.; vgl. dazu auch Art. 8 Abs. 1 der VO (EG) 141/2000 über Arzneimittel für seltene Leiden, ABl. L 18/1 ff. mit einer Garantie der Marktexklusivität für zehn Jahre. Auf diese Weise lässt sich etwa vermeiden, dass hohe Forschungs- und Entwicklungskosten zu unerwünschten Konzentrationen am Markt führen; vgl. auch Boom Nationale Regulierung bei internationalen Pharma-Unternehmen, 1993, $78 \mathrm{ff}$., $99 \mathrm{f}$.

${ }_{140} \mathrm{~S}$. etwa Blazejczak/Edler/Hemmelskamp/Jänicke ZfU 99, 1, 12ff., 28f.; Klemmer/ Lehr/Löbbe (Fn. 133), 111 f.; Kuhlmann/Bättig/Cuhls/Peter (Fn. 135), 43 ff., 121 ff.; Sachverständigenrat (Fn. 22), Tz. 51 ff.; Gawel/Lübbe-Wolff in: dies. (Fn. 108), 3, 5f.; Gawel ebd., 237, 296ff.; grundlegend zum Einfluss des Rechts auf Innovationen Porter Nationale Wettbewerbsvorteile, 1991, $665 \mathrm{ff}$;; s. auch Schulze-Fielitz in: Hoffmann-Riem/Schneider (Fn. 65), 312 ff.

${ }_{141}$ Dazu Blazejczak/Edler/Hemmelskamp/Jänicke(Fn. 140), $12 \mathrm{ff}$.

${ }_{142}$ Dazu Schulze-Fielitz in: Hoffmann-Riem/Schneider (Fn. 65), 313; Hapke/Japp (Fn. 105), 63 ff.; Zimmermann/Pahl in: Hansjürgens (Fn. 52), 15ff., 107; Schwarze in: Ott/
} 
tungspflichten, die die Anpassung des Produktdesigns an neue Erkenntnisse sichern. ${ }^{143}$ Vermeidungsanreize setzt auch die - allerdings nur für einzelne Stoffe praktikable - Ausgabe von handelbaren Emissionszertifikaten $^{144}$ und die ordnungs-, haftungs- und vergaberechtlichen Begünstigungen $^{145}$ der Teilnahme am EMAS-System, ${ }^{146}$ das zur Weiterentwicklung des betrieblichen Umweltmanagements verpflichtet. ${ }^{147}$ Auch Abwendungs- und Kompensationsbefugnisse ${ }^{148}$ und Selbstverpflichtungen ${ }^{149}$ sind zur Risikominimierung einsetzbar. ${ }^{150}$

Schäfer (Hrsg.) Die Präventionswirkungen zivil- und strafrechtlicher Sanktionen, 1999, $206 \mathrm{ff}$.

${ }^{143}$ BGH NJW 1990, 906, 907f.; dazu Vieweg in: Schulte (Fn. 3), 341; Kötz/Wagner Deliktsrecht, 9. Aufl. 2001, $172 \mathrm{ff}$.

${ }_{144}$ Dazu Grünbuch der Kommission ,zum Handel mit Treibhausgasemissionen der europäischen Union“, KOM (2000) 87 endg. vom 8.3.2000; Vorschlag für eine Richtlinie über ein System für den Handel mit Treibhausgasemissionsberechtigungen in der Gemeinschaft, KOM (2001) 581 endg.; zum Stand der Umsetzung in Deutschland www.bmu.de/de/1024/js/sachthemen/emission/aktuell_emission/; grundsätzlich befürwortend Sachverständigenrat (Fn. 22), Tz. 467 ff.; Hansjïrgens/Fromm ZfU 1994, $473 \mathrm{ff}$.; Cansier NVwZ 1994, 642 ff; Rengeling DVBI. 2000, 1473, 1481; Koch/Wieneke DVBI. 2001, 1085, 1091 ff.; einschränkend Zimmermann/Pahl in: Hansjürgens (Fn. 52), 107, 113 mit dem Hinweis, dass Zertifikate eine bereits bekannte Emission voraussetzen und gerade nicht zur Generierung neuen Risikowissens anregen.

${ }^{145}$ Dazu $\$ 21$ h WHG, $\$ 55$ a KrW-/AbfG, $\$ 58$ e BImSchG; $\$$ 3ff. EMASPrivilegV; zum Vergaberecht vgl. Interpretierende Mitteilung der Kommission über das auf das öffentliche Auftragswesen anwendbare Gemeinschaftsrecht und die Möglichkeiten zur Berücksichtigung von Umweltbelangen bei der Vergabe öffentlicher Auftrage, KOM (2001), 274 endg.; kritisch zu einer weitergehenden Begünstigung der Teilnehmer am UmweltAudit Koch in: Hoffmann-Riem/Schneider (Fn. 65), 273, 283f.; Sachverständigenrat (Fn. 22), Tz. $232 \mathrm{ff}$.

146 Environmental Management and Audit Scheme gem. Art. 1 VO (EG) 761/2001 über die freiwillige Beteiligung von Organisationen an einem Gemeinschaftssystem für das Umweltmanagement und die Umweltbetriebsprüfung (EMAS), ABl. L 114/1; zu dessen umweltrelevanten Effekten vgl. etwa Sachverständigenrat (Fn. 22), Tz. $106 \mathrm{ff}$.

147 Vgl. Anhang I-A.5. und 6., Anhang II 2.7., 2.8. VO (EG) 761/2001; dazu Boos Betriebliches Umweltmanagement als neues Modell der Risikosteuerung?, 2000, 159ff., $185 \mathrm{f}$.

148 Vgl. § 6 Abs. 1, 3 VerpackV; $\$ 7$ Abs. 3, § 17 Abs. 3a BImSchG; dazu Beaucamp Das Konzept der zukunftsfähigen Entwicklung im Recht, 2001, 373ff.; Voßkuhle (Fn. 110), $188 \mathrm{ff}$.

${ }_{149}$ Kritische Evaluation der Selbstverpflichtung der Autoindustrie zu Kfz-Emissionen und zur Altautoentsorgung sowie des BDI zum Klimaschutz in: Sachverständigenrat (Fn. 22), Tz. 436f., 918 ff., 446.

${ }^{150}$ Dazu Hoffmann-Riem in: ders./Schmidt-Aßmann (Hrsg.) Öffentliches Recht und Privatrecht als wechselseitige Auffangordnung, 1996, 261, 335. 
Langfristorientierung bietet, hinreichendes Risikowissen vorausgesetzt, die ordnungs- und abgabenrechtliche Regulierung. ${ }^{151}$ Ihr Steuerungsziel kann, wie im Falle der kalifornischen Abgasgesetzgebung, durchaus über den gegenwärtigen Stand der Technik hinausgehen.152 Signalwirkung kommt dann vor allem der Risikokommunikation im Vorfeld der Regelsetzung zu. ${ }^{153}$ Das Beispiel der US-Arzneimittelzulassung zeigt, dass gerade anspruchsvolle Zulassungsverfahren die Gewähr für hohe Akzeptanz und spätere Rentabilität bieten ${ }^{154}$ und dann zur Bildung eines „führenden Marktes" beitragen können, ${ }^{155}$ dessen Standards sich häufig in weniger streng regulierenden Exportländern durchsetzen. ${ }^{156}$ In einer global „entgrenzten Ökonomie“ kann sich ein Standort auf diese Weise wichtige, schwer transferierbare Kernkompetenzen sichern. ${ }^{157}$ Andernfalls muss er dem grenzüberschreitenden Risikotransfer durch Mitwirkung an internationaler Standardsetzung entgegenwirken.158

151 Wegner (Fn. 19), 37f,; Schmidt in: Hoffmann-Riem/Schmidt-Aßmann (Fn. 47), 67, 69; Blazejczak/Edler/Hemmelskemp/Jänicke (Fn. 140), 1ff.; Driesen The Economic Dynamics of Environmental Law, 2003; zur Erwartungssicherheit als Anforderung der Wirtschaft an das Recht Kuhlmann/Bättig/Cuhls/Peter (Fn. 135), 43 ff.; Hoffimann-Riem in: Eifert/Hoffmann-Riem (Fn. 138), 26ff.; zur ambivalenten Wirkung von Grenzwerten Engel in: Rengeling (Fn. 84), 17, 34f.

152 Sachverständigenrat (Fn. 22), Tz. 196.

153 Sachverständigenrat (Fn. 22), Tz. 52; Umweltbundesamt (Fn. 139), 361 ff.

154 Meyer-Krahmer in: Grimmer/Kuhlmann/Meyer-Krahmer (Hrsg.) Innovationspolitik in globalisierten Arenen, 1999, 43, 68f.; s. auch Kuhlmann/Bättig/Cuhls/Peter (Fn. 135), $17 \mathrm{ff}$.

155 Porter (Fn. 140), 666f.; zu den weiteren Kriterien der Innovationspolitik und Standortwahl internationaler Unternehmen Meyer-Krahmer (Fn. 154), $47 \mathrm{ff}$.

156 Scharpf Regieren in Europa: Effektiv und demokratisch?, 1999, 85ff.; Der Rat von Sachverständigen für Umweltfragen (Hrsg.) Zur Wirtschaftsverträglichkeit der Reform der Europäischen Chemikalienpolitik, www.umweltrat.de/03stellung/downlo03/stellung/Stellung_Reach_Juli2003.pdf, 21.

${ }^{157} \mathrm{Zu}$ den dafür erforderlichen Bedingungen näher Meyer-Krahmer (Fn. 154), $59 \mathrm{ff}$; Martinsen in: Grimmer/Kuhlmann/Meyer-Krahmer (Fn. 154), $135 \mathrm{ff}$.

158 Rublick Der grenzüberschreitende Transfer von Umweltrisiken im Völkerrecht, 1993, $105 \mathrm{ff}$;; auch der Vereinheitlichung der grundlegenden Sicherheitsanforderungen und technischen Spezifikationen im Binnenmarkt selbst kommt eine innovationsfördernde und bei entsprechendem Schutzniveau auch risikominimierende Rolle zu, dazu Stoll (Fn. 50), 218ff. 


\section{Das Recht der reflexiven Risikosteuerung}

\section{Gemeinschafisrechtliche Grundlagen}

Weite Teile des Risikoverwaltungsrechts sind - auch deshalb - heute mittelbar oder unmittelbar gemeinschaftsrechtlich geprägt.159 Primärrechtliche Grundlage ist vor allem ${ }^{160}$ die Verpflichtung der Gemeinschaft zur Vorbeugung und Vorsorge. ${ }^{161}$ Dabei betrifft die Vorsorge das Einschreiten bei begründetem Gefährlichkeitsverdacht und steuert damit das Risiko. ${ }^{162}$

Ihr Ziel ist die Herstellung eines hohen Schutzniveaus für Gesundheit, Sicherheit, Umwelt- und Verbraucherschutz gem. Art. 174 Abs. 2 S. 1, Art. 152 Abs. 1, Art. 95 Abs. 3 EGV. ${ }^{163}$ Die dabei gebotene Abwägung

159 Zur gemeinschaftsrechtlichen Regulierung von Umweltrisiken vgl. Falke Institutionen zur Risikobewertung und zum Risikomanagement im In- und Ausland, Studie im Auftrag der Ad-hoc-Kommission „Neuordnung der Verfahren und Strukturen zur Risikobewertung und Standardsetzung im gesundheitlichen Umweltschutz der Bundesrepublik Deutschland“, 2002, 60ff., www.bmu.de/de/1024/js/download/b_rk/liste.php; allgemein zum gemeinschaftsrechtlichen Umweltrecht auch die Beiträge in Erbguth (Hrsg.) Die Europäisierung des nationalen Umweltrechts, 2001; Epiney Umweltrecht in der Europäischen Union, 1997, 99ff.; ferner die Beiträge von Krämer in: Rengeling (Hrsg.) Handbuch zum europäischen und deutschen Umweltrecht, Bd. II, 1998, \$\$ 14 - 16; speziell zum Technikrecht Röthel in: Schulte (Fn. 3), $155 \mathrm{ff}$.

${ }^{160} \mathrm{Zu}$ den in der Entfaltung befindlichen grundrechtlichen Schutzpflichten Szczekalla in: Rengeling (Fn. 159), § 12 Rn. 20; Kingreen in: C. Calliess/Ruffert (Hrsg.) Kommentar zu EU-Vertrag und EG-Vertrag, 2. Aufl. 2002, Art. 6 EUV Rn. 46 ff.; Jaeckel Schutzpflichten im deutschen und europäischen Recht, 2001, 215 ff; Köck in: Rengeling (Hrsg.) Umgestaltung des deutschen Chemikalienrechts durch europäische Chemikalienpolitik (im Erscheinen), MS, $12 \mathrm{f}$.

${ }_{161}$ Diese sind über den Sachbereich des Art. 174 Abs. 2 EGV hinaus mittlerweile als allgemeine Rechtsprinzipien des Gemeinschaftsrechts anerkannt; dazu C. Calliess (Fn. 35), 197ff.; Röthel in: Schulte (Fn. 3), 155, 167 f.; Wägenbaur EuZW 2000, 162; vgl. auch EuGH Rs. C-157/96 (National Farmers Union), Slg. 1998, I-2211, 2259; Rs. C-180/96 (Vereinigtes Königreich/Kommission), Slg. 1998, I-2265, 2298; ferner Kommission (Fn. 16), 2; Vorbeugung zielt dabei auf die Abwehr von Gefahren im Sinne der deutschen Dogmatik; dazu Jans/v. d. Heide Europäisches Umweltrecht, 2003, 39; C. Calliess in: ders./Ruffert (Fn. 160), Art. 174 EGV Rn. 26 ff.; Epiney (Fn. 159), 99f.

162 Vgl. EuGH Rs. C-180/96 v. 5. 5. 1998 (Vereinigtes Königreich/Kommission), Slg. I-1998, 2265 Rn. 99: „Wenn das Vorliegen und der Umfang von Gefahren für die menschliche Gesundheit ungewiss ist, können die Organe Schutzmaßnahmen treffen, ohne abwarten zu müssen, dass das Vorliegen und die Größe dieser Gefahren klar dargelegt sind."; EuG Rs. T-125/96 v. 15. 1. 1998 (Simon/Hauptzollamt Frankfurt), Slg. II-1998, 00145; Rs. T-152/96 v. 1. 12. 1999 (Boehringer) Slg. II-1999, 03427 und Rs. T-13/99 v. 11. 9. 2002 (Pfizer/Animal Health), Slg. II-2002, 03305; dazu Epiney (Fn. 159), 100; Kommission (Fn. 16), 3f., 15.

163 S. etwa $C$. Calliess (Fn. 35), $136 \mathrm{ff}$. 
mit gegenläufigen, grundrechtlich geschützten Interessen ${ }^{164}$ haben die Gemeinschaftsorgane gem. Art. 174 Abs. 3 EGV nach umfassender Erhebung der vorhandenen Daten in einer nicht auf ökonomische Aspekte beschränkten Bewertung von Kosten und Nutzen unter Beachtung der Verhältnismäßigkeit zu treffen. ${ }^{165}$ Die Abwägung bezieht sich gem. Art. 174 Abs. 3, 3. Spiegelstrich EGV auch auf die Chancen und Risiken der Risikosteuerung selbst. ${ }^{166}$ Das hiernach angemessene Schutzniveau und die zur Vorsorge erforderlichen Maßnahmen sind nach Auffassung der EG-Kommission, der der EuGH insoweit einen weiten Gestaltungsspielraum einräumt, ${ }^{167}$ unter Abwägung der Folgen mit der größten Wahrscheinlichkeit zu bestimmen. ${ }^{168}$

Kennzeichnend für die sekundärrechtliche Ausgestaltung der Vorsorge sind neben materiellrechtlichen Qualitätsnormen und Beschränkungen ${ }^{169}$ vor allem die Prozeduralisierung der Risikosteuerung, ${ }^{170}$ die Harmonisierung der technischen Normung, ${ }^{171}$ die zunehmende Übertragung von Koordinations- und Durchführungsbefugnissen auf die Kommission ${ }^{172}$ und

\footnotetext{
${ }^{164} \mathrm{Zu}$ den grundrechtlichen Grenzen der Vorsorge $C$. Calliess (Fn. 53), $32 \mathrm{ff}$.

165 Kommission (Fn. 16), 3, 21.

${ }^{166}$ Dazu Schröder in: Rengeling (Fn. 159), § 9 Rn. 53; C. Calliess in: ders./Ruffert
} (Fn. 160) Art. 174 Rn. 41.

${ }^{167}$ EuGH C-180/96 (Vereinigtes Königreich/Kommission), Slg. I-1998, 2265 Rn. 62, 102 ff.; EuG Rs. T-70/99 (Alpharma/Rat), Slg. 2002, II-3497 Leitsätze 7 und 8 sowie Rn. 163 ff., 177 ff.; EuGH Rs. C-405/92 (Mondiet) Slg. 1993, I-6133 Rn. 31 f.; EuG Rs. T-13/99 (Pfizer/Animal Health), Slg. 2002, II-3305 Rn. 151, 166ff.; dazu Köck in: Rengeling (Fn. 160), 18, 23; C. Calliess (Fn. 53), 32ff.; v. Bogdandy JZ 2001, 157, $163 \mathrm{ff}$. ${ }_{168}$ Kommission (Fn. 16), 21; Williamson/Hulpke (Fn. 44), 93.

169 Dazu im Überblick Krämer in: Rengeling (Fn. 159), \$15 Rn. 33 ff.

${ }^{170}$ Dazu vor allem Art. 2 Abs. 1 der RL 85/337/EWG über die Umweltverträglichkeitsprüfung bei bestimmten öffentlichen und privaten Projekten, ABl. L 175/40.

$171 \mathrm{Vgl}$. Entschließung des Rates vom 7. 5. 1985 über eine neue Konzeption auf dem Gebiet der technischen Harmonisierung und der Normung, ABl. C 136/1; dazu Schulte in: Rengeling, (Fn. 159), § 17 Rn. 37ff.; Jörissen Produktbezogener Umweltschutz und technische Normen, 1997, 13f.; Sobczak Normung und Umweltschutz im Europäischen Gemeinschaftsrecht, 2002, $19 \mathrm{ff}$., $34 \mathrm{ff}$.; kritisch zum Stand der europäischen Normung Feldhaus UTR 54 (2000), 169, $186 \mathrm{ff}$.

172 Zur Institutionalisierung des Informationsaustauschs zwischen Kommission, Mitgliedstaaten und Industrie vgl. Art. 16 Abs. 2 RL 96/61/EG; zur Abstufung von Risikoermittlung und Genehmigungsverfahren zwischen Mitgliedstaaten und Kommission vgl. Art. 7 RL 93/67 EWG; Art. 9 ff. RL 91/414/EWG über das Inverkehrbringen von Pflanzenschutzmitteln, ABl. L 230/1 und Art. 3, 27f. RL 98/8/EG über das Inverkehrbringen von Biozid-Produkten, ABl. L 123/1; Art. 18 RL 2001/18/EG; Art. 5 , 6 Abs. 3 und 4 VO (EG) $258 / 97$ über neuartige Lebensmittel und neuartige Lebensmittelzutaten, ABl. L 43/1; Art. 10 ff. VO (EWG) 793/93; zur Übertragung einzelner Durchführungsbefugnisse auf die Kommission, vgl. Art. 50, 53 ff. VO (EG) 178/2002; zur vollen Übertragung 
die Beteiligung europäischer Behörden ${ }^{173}$ an der Risikoabschätzung. ${ }^{174}$ In Ablösung der eher statischen deutschen Technikorientierung ${ }^{175}$ zielt das Gemeinschaftsrecht damit neben der Rechtsangleichung vor allem auf eine schnelle Aktualisierung des Risikomanagements. ${ }^{176}$

\section{Verfassungsrechtliche Grundlagen}

\section{a) Zielsetzung und Voraussetzungen}

Das Grundgesetz verpflichtet zur Definition und Durchsetzung angemessener Einwirkungsgrenzen auf individuelle und kollektive Güter und zur Sicherung eines größtmöglichen Rahmens wirtschaftlicher Entfaltung. ${ }^{177}$ Die Schutzwürdigkeit der Risikoverursacher ist dabei vor allem davon abhängig, ob sich ihnen technisch durchführbare Maßnahmen zur Risikoreduktion oder zumutbare und risikoärmere Alternativen zur Zielerreichung anbieten. ${ }^{178}$ Auf Seiten der Risikoträger sind vor allem die

der Zulassung an die Gemeinschaft vgl. Art. 10, 73 vO (EWG) 2309/93 zur Festlegung von Gemeinschaftsverfahren für die Genehmigung und Überwachung von Human- und Tierarzneimitteln und zur Schaffung einer Europäischen Agentur für die Beurteilung von Arzneimitteln, ABl. L 214/1; Art. 8, 36 Vorschlag für eine Verordnung über genetisch veränderte Lebens- und Futtermittel KOM 2001, 425 endgültig.

${ }^{173}$ So die Europäische Umweltagentur (EEA), vgl. vO (EWG) 1210/90 zur Errichtung einer Europäischen Umweltagentur und eines Europäischen Umweltinformations- und Umweltbeobachtungsnetzes, AB1. L 120/1; die Europäische Agentur für die Beurteilung von Arzneimitteln, vgl. vO (EWG) 2309/93 und die Europäische Behörde für Lebensmittelsicherheit, vgl. VO (EWG) 178/2002.

174 Dazu kritischer Überblick bei Breuer NVwZ 1997, 833 ff.; ferner Kommission (Fn. 16), 18; Helberg in: Koch (Hrsg.) Umweltrecht, $2002 \S 3$ Rn. 39; Köck in: Rengeling (Fn. 160), $23 \mathrm{f}$.

$175 \mathrm{Zu}$ weiteren Unterschieden zwischen dem deutschen und dem gemeinschaftsrechtlichen Vorsorgeprinzip zuletzt Winter ZUR 2003, 137, 143.

176 Vgl. Art. 13 Abs. 1 RL 96/61/EG; dazu Salje in: Schulte (Fn. 3), 73, 103; ferner Art. 8 Abs. 1 und Anhang II RL 2001/18/EG; Art. 19 Abs. 1 VO (EG) 178/2002; dazu Kommission (Fn. 16), 23; Jans/v. d. Heide (Fn. 161), 37; Lübbe-Wolff(Fn. 48), 65; zur Statik des deutschen Technikrechts Bücker (Fn. 125), 93 ff.

$177 \mathrm{Zu}$ den grundrechtlichen Vorgaben im Überblick Schulte in: Kloepfer (Fn. 135), 59, 64. Im Rahmen der Forschungsfreiheit stellt sich die Frage, inwieweit diese auch die Inanspruchnahme von Rechtsgütern Dritter im Zuge der Forschungstätigkeit umfasst. Die gleichzeitige Gewährleistung des Eigentums und der damit verbundenen Nutzungsbefugnisse deutet darauf hin, dass das Grundgesetz die Träger von Freiheitsrechten nicht von der allgemeinen Güterzuordnung der Rechtsordnung freistellt, die grundrechtliche Ausübungsbefugnis also die Beeinträchtigung von Gütern und Interessen Dritter nicht umfasst; vgl. Scherzberg (Fn. 41), 510f.; ähnlich Wahl UTR 14 (1991), 8, $33 \mathrm{ff}$.

178 Ausführlich zu den Elementen der Abwägung Köck (Fn. 39), 37 ff., 259 ff.; Inhalt, Gegenstand und Ziele der grundrechtsgeschützten Betätigung unterliegen im Übrigen in- 
Freiwilligkeit der Risikoübernahme und die Fähigkeit zu selbstverantwortlichem Umgang mit dem Risiko erheblich. ${ }^{179}$ Der vom Grundgesetz geforderte Ausgleich individueller und kollektiver Güter zielt mithin darauf, die Handlungsoptionen im Umgang mit dem Risiko insgesamt zu erhöhen. ${ }^{180}$

Die aus den grundrechtlichen Schutzpflichten des Grundgesetzes folgende Vorsorge setzt keine sichere Schadensprognose voraus, sondern umfasst wie im Gemeinschaftsrecht alle Fälle wissenschaftlich begründeter Besorgnis. ${ }^{181}$ Risiken, deren Verwirklichung nach dem Stand von Wissenschaft und Technik praktisch ausgeschlossen erscheinen, sollen nach überwiegender Auffassung Vorsorge nicht erfordern und als ,sozial-adäquate Lasten“ von den Betroffenen zu tragen sein. ${ }^{182}$ Unter Ungewissheit führt dieser zweifache Verweis auf die Wissenschaft indes ins Leere. Die Notwendigkeit normativer Risikokonstitution ergibt sich ja gerade daraus, dass es hier auch der Wissenschaft an verlässlichen Beurteilungsmaßstäben fehlt. ${ }^{183}$ Grundrechtliche Schutzpflichten verlangen deshalb, mit der Eröffnung eines Risikos zugleich die Generierung neuen Risikowissens ${ }^{184}$ und die fortlaufende Evaluation der Risikoentwicklung zu gewährleisten. ${ }^{185}$ Sie gestatten die Begründung eines (Rest)Risikos nur, soweit und solange die Risiken der Risikoreduktion überwiegen. ${ }^{186}$

dividueller Maßstabswahl und keiner immanenten Relativierung anhand des „gesellschaftlichen Bedarfs"; dazu v. d. Daele in: Lege (Fn. 20), 119 ff.; Stoll (Fn. 50), 340; Groß VerwArch. 88 (1997), 89, 94ff.; dem Gesetzgeber steht allerdings bei der Definition und Gewichtung kollidierender Interessen ein weiter Einschätzungsspielraum zu, dazu sogleich IV.2.b).

${ }^{179}$ Diese bedarf vor allem verlässlicher informationeller Grundlagen, dazu auch unten IV.3.b)(bb)(4); ferner Kloepfer (Fn. 7), 36; Wolf in: Bora (Fn. 26), 65, 69f.; Huber UTR 36 (1996), 459, 484ff.; s. auch Stoll (Fn. 50), $357 \mathrm{ff}$.

${ }^{180}$ Zum Gesichtspunkt der Sozialverträglichkeit des Risikos dabei Pitschas in: Kloepfer (Fn. 134), 73, $76 \mathrm{ff}$.

181 Dazu etwa Bryde (Fn. 88), 81; Di Fabio DöV 1995, 1, 7f.; Schröder in: Schulte (Fn. 3), 185, 192; C. Calliess (Fn. 35), 319, $207 \mathrm{ff}$.

182 Grundlegend BVerfGE 49, 89, 143. Die Konkretisierung dieser Grenze der grundrechtlichen Verpflichtung zur Risikovorsorge setzt allerdings eine Abwägung voraus, für die der vom Bundesverfassungsgericht nominierte Maßstab der „praktischen Vernunft“ keine geeigneten Kriterien abgibt vgl. BVerfGE 49, 89, $133 \mathrm{ff}$; kritisch zur Rechtsprechung etwa Schröder in: Schulte (Fn. 3), 185, 194; Voßkuhle (Fn. 110), $382 \mathrm{f}$.

${ }_{183}$ Dazu bereits oben III.2.a); ferner Bryde (Fn. 88), 81 f.; Winter (Fn. 62), 115.

${ }^{184}$ In Betracht kommt hierzu Forschungsförderung, eigene staatliche Risikoforschung oder die Inpflichtnahme des Betreibers; s. dazu Hecht in: Hansjürgens (Fn. 52), $123 \mathrm{ff}$. 185 Stoll (Fn. 50), 282f.; vgl. auch Di Fabio (Fn. 181), 9; Wahl/Appel (Fn. 23), 41 ff.

${ }^{186}$ Bei der Entscheidung über die Wahrnehmung grundrechtlicher Schutzpflichten räumt das Bundesverfassungsgericht dem Gesetzgeber zwar, wie zuletzt zum Elektrosmog, einen Einschätzungsspielraum ein; vgl. BVerfGE 56, 54, 77f.; 92, 26, 46; BVerfG 
Auch jenseits grundrechtlicher Schutzpflichten setzt das Grundgesetz dem ,industriegesellschaftlichen Prozess“187 Grenzen. Der Umweltstaat, dem Art. 20a GG die Verantwortung für die natürlichen Lebensgrundlagen überträgt, muss auch jene alltäglichen Beanspruchungen der Umweltgüter problematisieren, deren Summations- und Fernwirkungen die Regenerationsfähigkeit des Naturhaushalts erst in der weiteren Zukunft zu zerstören drohen. ${ }^{188}$ Damit aber erscheinen zunehmend die Stoff-, Produkt- und Energieströme des gesellschaftlichen Güterverkehrs insgesamt als Problem. ${ }^{189} \mathrm{Im}$ Rahmen des Art. 20a GG kommt es insoweit weder auf den Verdacht konkreter Schädlichkeit noch auf das Überschreiten der Vorsorgeschwelle an. Stattdessen ist das rechtliche Reaktionsvermögen unter Ungewissheit als solches zu erweitern und sind unerkannte Risiken durch eine „prinzipale Reduktion der Umwelteinwirkungen“ zu minimieren. ${ }^{190}$ Dem Umweltstaat obliegt also auch die „Zukunftsvorsorge“ gegenüber noch unbekannten Eventualitäten. ${ }^{191}$

DÖV 2002, 521, 521f.; BVerwG UPR 1998, 393f.; für den Jugendschutz auch BVerfGE 83, $130,140 \mathrm{ff}$;; auch der Gesetzgeber darf aber, was unsicher und umstritten ist, nicht einfach ignorieren, sondern muss die Ungewissheit in seine Bewertung einfliessen lassen und auf sie reagieren; s. Köck ZUR 2002, 349 ff.; tatsächlich geht der Gesetzgeber bei der Regelung der sog. Dennoch-Störfälle in $\S 3$ Abs. 3 StörfallV (und $\S 7$ Abs. $2 a$ AtG a. F.) über das auf der Basis derzeitiger Schutzpflichtdogmatik geltende Maß der Risikominimierung hinaus; s. Breuer WiVerw 1981, 219, 239f.; a. A. Hansmann in: Landmann/Rohmer, Umweltrecht Bd. II (Bearb. Stand März 2002), § 3 12. BImSchV Rn. 24.

187 Badura UTR 43 (1998), 43, 44.

188 Schmidt-Aßmann (Fn. 49), 3/72; ferner auch Hofmann in: Bizer/Koch (Fn. 48), 101, $105 \mathrm{ff}$.

189 Schmidt-Aßmann (Fn. 49), 3/72.

190 So Wolf in: Bora (Fn. 26), 65, 81; s. auch Köck (Fn. 39), 199; in diesem Sinne wird Art. 20a GG vielfach ein Optimierungsgebot des Inhalts entnommen, dass der Gesetzgeber verpflichtet sei, den Schutz der natürlichen Lebensgrundlagen unter Berücksichtigung anderer Verfassungsziele „bestmöglich“ zu verwirklichen und dazu von mehreren gleichwertigen Handlungsalternativen diejenige zu wählen, die die natürlichen Lebensgrundlagen am wenigsten belastet; vgl. etwa Epiney in: v. Mangoldt/Klein/Starck (Hrsg.) GG, 4. Aufl. 2000, Art. 20a Rn. 62; Murswiek in: Sachs (Hrsg.) GG, 2. Aufl. 1999, Art. 20a Rn. 53; Wolf (Fn. 121), 303; Schulze-Fielitz in: Dreier (Hrsg.) GG, Bd. II, 1998, Art. 20a Rn. 23; Bernsdorff NuR 1997, 328, 332.

191 Dazu näher C. Calliess (Fn. 35), 114ff., 122; zur Offenhaltung der Zukunft aus philosophischer Sicht auch Ewald Soziale Welt 49 (1998), 5, 13 ff.; zum Begriff der Zukunftsvorsorge vgl. Bundesminister für Umwelt, Naturschutz und Reaktorsicherheit, Umweltbericht 1990, BTDrucks 11/7168, 26. 


\section{b) Modalitäten und Instrumente}

Die grundrechtlichen Übermaß- und Untermaßverbote ${ }^{192}$ sind unter Ungewissheit kaum operabel. ${ }^{193}$ Ihre materiellen Unterschiede werden durch den funktionalen Einschätzungs- und Gestaltungsspielraum des Gesetzgebers für Prognosen und Wertentscheidungen überlagert. ${ }^{194}$ Für die „Wirkungstiefe“ verfassungsrechtlicher Anforderungen ist es deshalb im Ergebnis unerheblich, ob die fragliche Maßnahme das Risiko für ein Grundrechtsgut durch Eingriff erhöht oder durch Schutzgewährung mindert. ${ }^{195}$ Freiheit und Sicherheit haben damit im Ergebnis - das knüpft an die gestrigen nachmittäglichen Referate an - denselben Rang, geht es ihnen doch um die ,eine“ reale Freiheit.

Bei seiner Mittelwahl ist der Gesetzgeber „weder auf marktferne ordnungsrechtliche noch auf marktkonforme, ökonomische ' Instrumente beschränkt“ ${ }^{196}$ Zwar mag sich aus den Freiheitsrechten eine Präferenz für die Aktivierung selbstregulativer Beiträge entnehmen lassen. ${ }^{197}$ Diese steht aber unter dem Vorbehalt der effektiven Wahrnehmung grundrechtlicher sowie sozial- und umweltstaatlicher Verantwortung. ${ }^{198}$ In beiden setzt sich das Grundgesetz von der ökonomischen Analyse des Rechts

${ }_{192}$ Zum Übermaßverbot bzw. Verhältnismäßigkeitsgrundsatz vgl. etwa BVerfGE 19 , 342, 348f.; 61, 126, 134; 76, 1, 50f.; 90, 145, 173; 96, 10, 23f.; zum Untermaßverbot s. BVerfGE 88, 203, 254; BVerfG NJW 1996, 651; Isensee in: ders./Kirchhof, Handbuch des Staatsrechts Bd. V, 1992, § 111 Rn. 165f.; Scherzberg Grundrechtsschutz und „Eingriffsintensität“, 1989, $208 \mathrm{fff}$; zum Verhältnis von Übermaß- und Untermaßverbot Dietlein ZG 1995, 131, $136 \mathrm{ff}$; Erichsen Jura 1997, 85, 88; Dolderer Objektive Grundrechtsgehalte, 2000, 270ff.; C. Calliess (Fn. 35), 457f.; Ruffert Vorrang der Verfassung und Eigenständigkeit des Privatrechts, 2001, $215 \mathrm{ff}$.

${ }_{193}$ Dazu Rehbinder FS Sendler, 2. Aufl. 1997, 269, 279; Spiecker gen. Döhmann (Fn. 20), 80f.; Lübbe-Wolff(Fn. 48), 62ff.; Köck AöR 121 (1996), 1, 17.

${ }_{194}$ BVerfGE 77, 381, 403; 79, 174, 202; 88, 203, 254, 262; kritisch zur Rechtsprechungspraxis Scherzberg DVBI. 1999, 356, 362f.; Beispiele für eine Überschreitung des Gestaltungsspielraums bei Rehbinder NuR 1997, 313, 326.

195 Dem Grundgesetz ist deshalb im Ergebnis kein „omission bias“ eigen, die bei menschlichem Entscheidungsverhalten beobachtete Präferenz für riskantes Unterlassen gegenüber riskantem Tun; dazu vgl. Ritov/Baron Journal of Behavioral Decision Making 3 (1990), $263 \mathrm{ff}$; Sunstein University of Chicago Law Review 70 (2003), 751, $779 \mathrm{ff}$. Dem Gesetzgeber dürfte eine Bevorzugung riskanten Unterlassens - wie im Arzneimittelrecht - aber auch nicht grundsätzlich verwehrt sein, dazu Köck (Fn. 39), 114f.; zur Erweiterung gesellschaftlicher Handlungsmöglichkeiten als Ziel der Risikosteuerung bereits oben III.2.c).

196 Steinberg (Fn. 13), 168, Köck in: Gawel/Lübbe-Wolff (Fn. 108), 323, 343 ff.

197 Schmidt-Preuß (Fn. 47), 170f.; s. auch Stoll (Fn. 50), 346, 362f.

198 Mrasek-Robor (Fn. 50), 205ff.; auf sozialstaatliche Verpflichtungen der Techniksteuerung weist etwa Pitschas in: Kloepfer (Fn. 134), 73, 82ff. hin. 
$a b$, die eine Minderung des Rechtsgüterbestandes teilweise schon dann als vorteilhaft bewertet, wenn der Begünstigte die Veränderung trotz Verpflichtung zur Entschädigung des Benachteiligten anstrebt. ${ }^{199}$ Rechtsgüterschutz lässt sich nicht auf finanzielle Kompensation reduzieren. ${ }^{200}$ Grundrechtliche Schutzaufträge sind deshalb auch durch die Begründung von Haftungs- und Versicherungspflichten allein nicht erfüllbar. ${ }^{201}$

Soweit die mangelnde Regelungsreife der Materie und der Zwang zur Dynamisierung der Vorsorge einer inhaltlichen Maßstabsbildung entgegenstehen, ${ }^{202}$ ist die Erreichung verfassungsrechtlicher Schutzziele prozedural zu gewährleisten. ${ }^{203}$ Zur Sicherung eines angemessenen Risikomanagements ${ }^{204}$ gehört dabei nicht zuletzt die Standardisierung der bisher ungeregelten Verfahren und Konventionen der Risikoabschätzung ${ }^{205}$ sowie die Gewährleistung einer fortlaufenden Evaluation des Steuerungserfolgs. ${ }^{206}$ Diese Pflicht zum „Monitoring“ umfasst nicht nur die Folgenbeobachtung durch den Gesetzgeber, sondern auch die Weitergabe entsprechender Revisionspflichten an Normkonkretisierung und -vollzug. 207

199 Schäfer/Ott Lehrbuch der ökonomischen Analyse des Zivilrechts, 3. Aufl. 2000, $32 \mathrm{ff}$.

200 Dazu Wolf KJ 1989, 55ff.; Damm in: Bora (Fn. 26), 93, 101 f.; Voßkuhle (Fn. 110), 381 ff.; vgl. auch Britz Die Verwaltung 30 (1997), 185, 206ff; Lübbe in: Gawel/LübbeWolff (Fn. 108), 13, 25 ff.; für den Eigentumsschutz Scherzberg (Fn. 80), 40; für den Umweltschutz Wolf(Fn. 121), 295; s. auch $\$ 19$ Abs. 1 und 2 BNatSchG.

201 Schmidt-Aß3mann in: Hoffmann-Riem/Schmidt-ABmann (Fn. 150), 7, 22; zur vergleichsweise geringen Wirkungsschärfe des Haftungsrechts Ekardt/Manger/Neuser/Pottschmidt/Roßnagel/Rust (Fn. 76), 169ff.; Hapke/Japp (Fn. 105), 79f., 91 ff., 100ff.; jedenfalls bei Risiken mit hohem und hinreichend sicher erkennbarem Schadenspotential wie im Gentechnik-, Atom-, Immissionsschutz-, Chemikalien- und Lebensmittelrecht ist deshalb die Nutzung regulatorischen Rechts unverzichtbar; dazu Steinberg (Fn. 13), 170. Schulze-Fielitz in: Hoffmann-Riem/Schneider (Fn. 65), 291, 327 weist zu Recht darauf hin, dass es "sehr konkret vor allem auf die ökonomischen Rahmenbedingungen ankommt, ob und inwieweit das ,hard law" des ordnungsrechtlichen Instrumentariums ergänzt oder gar ersetzt werden kann; ebenso Sachverständigenrat, (Fn. 22), Tz. 34*, $192 \mathrm{ff}$.

${ }^{202}$ Vgl. BVerfGE 49, 89, $137 \mathrm{ff} ., 140 \mathrm{ff}$;; zu den Möglichkeiten materieller Programmierung ausführlich Steinberg (Fn. 13), $205 \mathrm{ff}$;; Schulze-Fielitz (Fn. 3), $469 \mathrm{ff}$.

${ }^{203}$ Dazu Lübbe-Wolff(Fn. 48), 65 m.w.N.; Stoll (Fn. 50), 325; Di Fabio (Fn. 62), 465 f.

${ }^{204} \mathrm{Zu}$ den insoweit zu stellenden Anforderungen Scherzberg (Fn. 41), $499 \mathrm{ff}$;; zu den Elementen des Risikomanagements ferner Henschler UTR 43 (1998), 11, 18ff.; Seiler (Fn. 111), 30ff.; Gill/Bizer/Roller (Fn. 40), 81 ff.; vgl. dazu auch den Abschlussbericht der Risikokommission (Fn. 52), $23 \mathrm{ff}$.

${ }^{205}$ Vgl. dazu den Vorschlag der Risikokommission, Abschlussbericht (Fn. 52), 71 ff.; dazu bereits oben III.2.a).

206 Wolf(Fn. 121), 296f.; Schulze-Fielitz (Fn. 3), 485f.; vgl. auch BVerfGE 88, 203, 309 ff. ${ }^{207}$ Steinberg (Fn. 13), $172 \mathrm{f}$. 


\section{Die Risikosteuerung im Risikoverwaltungsrecht}

Die Komplexität europarechtlicher Vorsorgeanforderungen, grundrechtlicher Schutzpflichten und umweltstaatlicher Verantwortung lässt sich auf zwei Aufgabenbereiche des Risikoverwaltungsrechts zusammenführen: Risikoreduktion bei hinreichend bekannten und Risikoprävention bei unbekannten oder nur vermuteten Risiken. ${ }^{208}$

\section{a) Die Risikoreduktion}

\section{(aa) Das Regelungsmodell}

Zur Risikoreduktion wird in den Tatbeständen von Gefahrenabwehr und Vorsorge die Grenze der Belastbarkeit der betroffenen Güter definiert und über die Vermeidbarkeit ihrer Beeinträchtigung entschieden. ${ }^{209}$ Grundfragen wie die relevanten Schutzgüter, die Bewertung des Nutzens einer Innovation, das medienübergreifende Ziel der Risikominimierung, deren Begrenzung auf die „beste verfügbare Technik“210 sowie Art und Umfang des von der Verwaltung anzustellenden Risikovergleichs sind dabei gesetzlich zu regeln. ${ }^{211}$ Damit werden die Rechtsfragen reflexiver Risikosteuerung freilich nur in beschränktem Umfang abgeschichtet. Je mehr es um innovative, in Wirkung und Folgen ungewisse Anlagen, Stoffe oder Produkte geht, desto weniger vermögen abstrakte Vorgaben des Sicherheitsniveaus und Verweise auf den Stand der Wissenschaft und Technik die Risikoentscheidung zu determinieren. ${ }^{212}$ Für die Beurteilung der Nanotechnologie oder neuer chemischer Stoffe gibt es keinen vorfindlichen "Stand der Wissenschaft", ${ }^{213}$ für Infrastruktureinrichtungen

${ }^{208}$ Zur Unterscheidung von bekannten und unbekannten Risiken auch Hapke ZfRSoz 20 (1999), 55, 66; weiter differenzierend WBGU (Fn. 28), 6ff., der bekannte Risiken mit geringem Schadenspotential und geringer bis mittlerer Eintrittswahrscheinlichkeit (,Risiken im Normalbereich"), Risiken, die über das alltägliche Ausmaß deutlich hinausgehen, weil die Ungewissheit der Schadensentwicklung oder/und das Schadenspotential und die Eintrittswahrscheinlichkeit als hoch zu bewerten sind, („Risiken im Verbots- oder Grenzbereich") und „unbekannte Risiken“ unterscheidet. Für letztere schlägt er den Übergang auf eine präventive Technikentwicklungssteuerung vor, vgl. WBGU (Fn. 28), 6f., $306 \mathrm{ff}$.; das entspricht der nachstehend in litera b) erörterten Risikoprävention.

${ }^{209} \mathrm{Zu}$ dieser Charakterisierung von Gefahrenabwehr und Vorsorge Rehbinder (Fn. 50), $04 / 20$.

${ }^{210}$ Art. 2 Nr. 11 RL 96/61/EG; dazu instruktiv Feldhaus NVwZ 2001, 1 ff.

211 Zur Reichweite des Vorbehalts des Gesetzes in Ungewissheitssituationen grundlegend BVerfGE 49, 89, $139 \mathrm{ff}$; ferner Wahl/Appel (Fn. 23), $126 \mathrm{ff}$.

212 Steinberg (Fn. 13), 430f.

$213 \mathrm{Vgl}$. VGH BW VBIBW 2002, $436 \mathrm{ff}$. zur Nanotechnologie; allgemein zum Verhältnis von Theorie und Anwendung Kleindiek (Fn. 43), $102 \mathrm{ff}$. 
und andere großtechnische Anlagen keinen abrufbaren „Stand der Technik", 214 nach dem die maßgeblichen Beurteilungen vorgenommen werden könnten. ${ }^{215}$ Die Risikosteuerung wird insoweit auf wissensgenerierende administrative Verfahren delegiert. ${ }^{216}$

\section{(bb) Die Maßstabsbildung im Verfahren}

In ihnen werden Umweltstandards ${ }^{217}$ und Vorsorgekonzepte ${ }^{218}$ erstellt und in fallbezogenen Zulassungsentscheidungen realisiert. ${ }^{219}$ Privates Risikowissen fließt dabei über die Anhörung der beteiligten Kreise, die kontrollierte Anknüpfung an technische Standards 220 und die Auswertung der vom Vorhabenträger im Zulassungsverfahren beizubringenden Prüfnachweise und Risikobewertungen ein. ${ }^{221}$ Die wissenschaftlichen Entscheidungsgrundlagen werden durch die Beteiligung beratender Kommissionen und Ausschüsse und durch die Beauftragung von Sachverständigen bei der "nachvollziehenden“ Amtsermittlung222 gewon-

${ }^{214}$ Ekardt/Manger/Neuser/Pottschmidt/Roßnagel/Rust (Fn. 76), 119f., 123f., $283 \mathrm{ff}$.

215 Ladeur in: Hoffmann-Riem/Schmidt-Aßmann (Fn. 47), 111, 126; vgl. dazu etwa $\$ 4 \mathrm{e}$ Abs. 4 9. BImSchV, wonach bei der Zusammenstellung der Unterlagen bei UVP-pflichtigen Vorhaben auf fehlende Kenntnisse und Prüfmethoden hinzuweisen ist. Im Bereich der Informations- und Kommunikationstechnik werden heute teilweise informelle, provisorische technische Standards bereits im Wege „entwicklungsbegleitender Normung“ erstellt und damit dem formellen Normungsprozess faktisch entzogen, dazu Schulte in: Kloepfer (Fn. 135), $68 \mathrm{ff}$.

${ }^{216}$ Zur Delegation der Entscheidungsbefugnis im Risikoverwaltungsrecht bereits Scherzberg in: Engel/Halfmann/Schulte (Fn. 14), 113, 128 f., 140.

217 In den Rechtsformen der Rechtsverordnung und Verwaltungsvorschrift, dazu Steinberg (Fn. 13), $228 \mathrm{ff} ., 234 \mathrm{ff}$., dort auch zum Fehlen ausreichender gesetzlicher Strukturierung des Verfahrens; ferner Schulze-Fielitz (Fn. 3), $453 \mathrm{ff}$., $464 \mathrm{ff}$., $480 \mathrm{ff}$.

${ }^{218}$ Dazu Trute Vorsorgestrukturen und Luftreinhalteplanung im BImSchG, 1989, 66f.; Wahl/Appel (Fn. 23), 174ff.

219 Dazu im Kontext der IVU-Richtlinie Buchholz (Fn. 110), 134; Lübbe-Wolff NuR 1999, 241 ff.; eine implizite Delegation der Bewertung der Sicherheit und der Auswirkungen eines Vorhaben auf die Genehmigungsbehörde findet sich etwa in $\S 20$ Abs. 1 b 9. BImSchV; dazu auch unten IV.3.a)(dd).

220 Dazu Feldhaus (Fn. 171), 179 ff.; Schmidt-Preuß(Fn. 47), 203 ff.; zur Widerlegung der Vermutung der Richtlinienkonformität bei Einhaltung technischer Standards im Gemeinschaftsrecht Schulte in: Rengeling (Fn. 159), § 17 Rn. 74ff., Sobczak (Fn. 171), 49 ff.; kritisch zum Stand der europäischen Normung Feldhaus (Fn, 171), $186 \mathrm{ff}$.

221 Vgl. etwa $\$ \S 5$ f. UVPG, $\$ \$ 6$ ff. ChemG, $\$ 10$ Abs. 2 und $\$ 15$ Abs. 1 und 3 GenTG, $\$ 12$ Abs. 3 PflSchG, Art. 6 Abs. 1 VO EG 258/97; dazu auch die Empfehlung 97/618/EG der Kommission, ABI. L 253/1; zum Ganzen auch Stoll(Fn. 50), 280f.; Tünnesen-Harmes (Fin. 62), 216ff.

222 J.-P. Schneider Nachvollziehende Amtsermittlung bei der Umweltverträglichkeitsprüfung, 1991. 
nen. ${ }^{223}$ Angesichts der Wertungsabhängigkeit wissenschaftlicher Konventionen ist eine institutionelle Trennung von Risikoabschätzung und Risikomanagement angezeigt ${ }^{224}$ und unterliegen die Ergebnisse der Risikoabschätzung einer "steuernden Rezeption“.225 Der sozialen und psychologischen Dimension des Risikos entspricht es, die Bestimmung abstrakter Umweltstandards und die projektbezogene Sicherheitskonzeption zum Gegenstand öffentlicher Erörterung zu machen und deshalb transparent und partizipativ auszugestalten. ${ }^{226}$ Dabei kann soziale Risikokompetenz umso eher eingebunden werden, je mehr die Verfahren als Prozess sozialer Verständigung ausgestaltet sind und innerhalb oder außerhalb der formalisierten Abläufe von Anhörung und Erörterung persönliche Interaktionen erlauben. ${ }^{227}$ Prozedurale Rationalität zielt insoweit darauf, im Verfahren rechtliche, verwaltungspolitische, betriebliche, professionelle, wissenschaftlich-technische und soziale Perspektiven in optimaler Weise zu entfalten. ${ }^{228}$

\section{(cc) Die Verteilung der Beweislast}

Soweit sich die komplexen Wirkungsbeziehungen des Risikos im Verfahren nicht hinreichend sicher klären lassen, können stabile Vermu-

223 Allgemein dazu Steinberg (Fn. 13), 256ff., 264ff.; Überblick bei Tünnesen-Harmes (Fn. 62), $243 \mathrm{ff}$;; zu den Besonderheiten von Ethikkommissionen bei der klinischen Prüfung von Arzneimitteln G.-P. Calliess (Fn. 6), $236 \mathrm{ff}$.; zur ZKBS der $\$ 4 \mathrm{f}$. GenTG Tünnesen-Harmes (Fn. 62), 228 ff.; vgl. auch Schröder Kommissionskontrolle in Reproduktionsmedizin und Gentechnologie, 1992, $79 \mathrm{ff} ., 233 \mathrm{ff}$.

224 Vgl. National Research Council, Risk Assessment in the Federal Government, 1983, 3, 17 ff.; Der Rat von Sachverständigen für Umweltfragen, Umweltgutachten 1996, BTDrucks. 13/4108, $39 \mathrm{ff}$., $300 \mathrm{ff}$.

225 Dazu Trute (Fn. 46), 102f.; soweit die Abweichung von Kommissionsvoten einer besonderen Begründungspflicht unterliegt, vgl. $\S 10$ Abs. 7 S. 4 GenTG, $\S 25$ Abs. 6 S. 3 AMG, wird damit die Letztverantwortung der Behörde nicht in Frage gestellt; zum Problem der Verselbständigung des wissenschaftlich-technischen Sachverstandes SchulzeFieliz (Fn. 3) 443, 449f.; zur Notwendigkeit einer rechtlichen Verarbeitung wissenschaftlicher Abschätzungsunsicherheit bereits oben III.2.a).

226 Ausführlich dazu die Vorschläge im Abschlussbericht der Risikokommission (Fn. 52), 53 ff.; zur Informationsfunktion der Öffentlichkeitsbeteiligung allgemein Fisahn Demokratie und Öffentlichkeitsbeteiligung, 2002, 210f.; zur Informationsfunktion des Informationszugangsrechts vgl. Scherzberg (Fn. 47), $186 \mathrm{f}$.

${ }^{227}$ Dazu bereits Scherzberg(Fn. 47), 134f.; ferner die Beiträge in: Köberle (Hrsg.) Diskursive Verständigung? Mediation und Partzipation in Technikkontroversen, 1997. Gesetzliche Ansätze hierfür in $\S 71$ c Abs. 1 S. 1 und 2, § 71e VwVfG sowie $\S 2$ Abs. 2 S. 14. BImSchV.

228 Dazu Ekardt/Manger/Neuser/Pottschmidt/Roßnagel/Rust (Fn. 76), $283 \mathrm{ff}$,; vgl. zum BImSchG auch BVerfGE 98, 83, 89f. 
tungsregeln den Entscheidungsprozess entlasten. ${ }^{229}$ So kann eine Beschränkung an den Nachweis der Gefährlichkeit eines Stoffes oder eine Zulassung an den Nachweis seiner Ungefährlichkeit gebunden werden. ${ }^{230}$ Das hebt, wie nach dem US-Toxic Substances Control Act, ${ }^{231}$ die staatlichen Eingriffsschwellen oder, wie im Falle des deutschen Pflanzenschutzrechts, ${ }^{232}$ die Markteintrittsbarrieren für neue Produkte an. Ähnlich soll der Gesetzgeber nach den Vorschlägen der EG-Kommission zur Chemikalienregulierung ${ }^{233}$ bestimmte Stoffe einem generellen Gefährlichkeitsverdacht unterstellen und dem Unternehmer dessen Widerlegung für den jeweiligen Verwendungszweck im Rahmen eigener Risikoforschung auferlegen. ${ }^{234}$

Fehlt es an einer ausdrücklichen gesetzlichen Beweislastverteilung, kann abhängig vom Schutzzweck der Norm zu berücksichtigen sein, dass hohe Anforderungen an die Beweisführung vor allem bei unbekannten Stoffen und Anwendungen als negativer Anreiz für Risikoforschung und Innovation wirken. ${ }^{235}$ Den beteiligten grundrechtlichen und umweltstaatlichen Interessen ist deshalb durch eine Abstufung der Informationslasten Rechnung zu tragen. ${ }^{236}$ Die beiderseitige Wissensgenerierung wird gefördert, wenn es dem Risikoverursacher obliegt, diejenigen Schädlichkeitshypothesen zu falsifizieren, für die die Behörde begründete Anzeichen vorbringt. ${ }^{237}$

229 Dazu C. Calliess (Fn. 41), 1732; Spiecker gen. Döhmann in: Rengeling (Fn. 160), 35.

${ }^{230} \mathrm{Zu}$ letzterem vgl. § 15 Abs. 1 Nr. 3 lit. d PflSchG, dazu Köck (Fn. 39), 185 ff.

231 Vgl. \$§ 5, 6 TSCA; dazu Spiecker gen. Döhmann in: Rengeling (Fn. 160), $9 \mathrm{ff}$., ähnlich auch das früher in Großbritannien herrschende "good science"-Konzept, Steinberg in: Riedel (Fn. 46), 17, $22 \mathrm{ff}$.

${ }^{232} \mathrm{Vgl} . \S 15$ Abs. 1 Nr. 3 lit. b PflSchG; vgl. auch $\S 11$ Abs. 1 Nr. 4 und $\S 16$ Abs. 1 Nr. 3 GenTG; zu § 15 I Nr. 3 lit. b a. F. PfISchG BVerwGE 81, 12, 15; dazu Di Fabio (Fn. 62), 159f.; Köck (Fn. 39), 185ff.; allgemein für eine solche Ausgestaltung der Beweislast Schachtschneider in: Thieme (Hrsg.) Umweltschutz im Recht, 1988, 81, 120f.; Beck (Fn. 32), 274f., 291.

${ }^{233}$ Vgl. Weißbuch der EG-Kommission (Fn. 53), 19f.; s. auch bereits Kommission (Fn. 16), 25 f.

${ }^{234}$ Dazu C. Calliess (Fn. 53), 47 ff.; Appel UTR 68 (2003) 95, 108ff.; die Regelung vernachlässigt allerdings die umweltgefährlichen Stoffe, vgl. Appel ebd., 114f.; kritisch auch v. Holleben/Schmidt NVwZ 2002, 532, $535 \mathrm{f}$.

235 Ladeur in: Bora (Fn. 26), 56; Spiecker gen. Döhmann in: Rengeling (Fn. 160), 12; dagegen überzeugt die Kritik gegenüber einer Beweislastumkehr bei bereits auf dem Markt befindlichen Stoffen nicht, s. aber Williamson/Hulpke (Fn. 44), 92.

${ }^{236}$ Murswick (Fn. 126), 213ff.; Wahl/Appel (Fn. 23), 124; Köck in: Rengeling (Fn. 160), 35f.; C. Calliess (Fn. 41), 1732f.; zur Nivellierung der Bedeutung der präventiven und repressiven Regelungsstruktur für die Beweislastverteilung Di Fabio (Fn. 62), 204ff.

${ }^{237}$ So die Rechtslage im Immissionsschutzrecht, vgl. etwa Jarass BImSchG, 4. Aufl. 1999, § 6 Rn. 8, § 3 Rn. 28f.; zum Ganzen C. Calliess (Fn. 41), 1729 ff. 


\section{(dd) Die Zuweisung des Risikos}

Soll gesichert werden, dass rechtliche Risikosteuerung „more good than harm" bewirkt, ${ }^{238}$ kann dies vor dem Hintergrund der Lückenhaftigkeit des Risikowissens nicht quantitativ berechnet, sondern nur qualitativ entschieden werden. ${ }^{239}$

Freilich bedarf es für die „Berücksichtigung“ der insoweit im Verfahren gewonnenen Erkenntnisse eines rationalisierenden Maßstabs. ${ }^{240}$ Vorsorgeziel und Gleichheitssatz verpflichten insoweit auf Kohärenz. ${ }^{241}$ Kohärenz verknüpft langfristige Zielbestimmung mit kurzfristiger Mittelwahl und verlangt dazu eine in Konzepten formalisierte ${ }^{242}$ oder in Einzelentscheidungen fortgebildete ${ }^{243}$ administrative „Risikopolitik“.244 Nur auf dieser Grundlage lässt sich eine angemessene, raum-zeitliche Streuung von Risiken gewährleisten und dem Innovationsprozess zugleich Langfristorientierung bieten. ${ }^{245}$

\footnotetext{
238 Warren/Marchant (Fn. 44),

239 Kommission (Fn, 16), 22f.; EEB, Position on the Precautionary Principle, 1999, www.eeb.org/publication/eeb.; dazu auch Gollier (Fn. 124), $303 \mathrm{ff}$;; Schütz (Fn. 110), $108 \mathrm{ff}$; einen Versuch der Quantifizierung unternehmen Cansier/Cansier in: Hansjürgens (Fn. 52), $157 \mathrm{ff}$., ohne daß die Umwandlung der Komplexität der Bewertung in eine Komplexität der Berechnung einen Rationalisierungsgewinn erkennen ließe; zur Untauglichkeit der Kosten-Nutzen-Analyse bei Ungewissheit auch Hansjürgens in: Gawel (Fn. 57), $63,86 \mathrm{ff}$.

240 Wahl/Appel (Fn. 23), $46 \mathrm{f}$.

${ }^{241}$ Kommission (Fn. 16), 22; zur Kohärenz als Rationalitätsmerkmal der Entscheidung unter Ungewissheit Grunwald (Fn. 133), 286f.; allgemein dazu auch Strauch ThürVB1. 2003, 1, $4 \mathrm{ff}$.; ders. KritV 85 (2002), $311 \mathrm{ff}$.; Bracker Kohärenz und juristische Interpretation, 2000.

${ }^{242}$ Die Verpflichtung der Verwaltung zu Erstellung eines Konzepts im Umgang mit schwach belegtem Besorgnispotential befürwortet etwa Stoll (Fn. 50), 86; zum Erfordernis eines Konzepts zur Vorsorge gegen raum-zeitliche Fernwirkungen s. BVerwGE 69, 37, 45; Trute (Fn. 218), 66f.; Qualitätsanforderungen an die Risikocharakterisierung stellt auch die US Environmental Protection Agency (EPA) auf, dazu Wiedemann/Mertens/ Clauberg/Hennings/Schütz (Fn. 98), 68ff.

${ }^{243} \mathrm{Zu}$ Recht gegen eine Pflicht zur Formalisierung des Konzepts im Kontext des $\$ 5$ Abs. 1 Nr. 2 BImSchG Roßnagel in: Koch/Scheuing (Hrsg.) Gemeinschaftskommentar zum BImSchG, Stand April 2003, § 5 Rn. 524ff.

244 Dazu auch Murswiek (Fn. 126), 218f., 222f.; Di Fabio (Fn. 62), 454; Scherzberg (Fn. 47), 128; Trute(Fn. 46), 96; ein solches Konzept entwickelten die Behörden im Umgang mit der Nanotechnologie im Falle des VGH BW VBIBW 2001, 436ff. durch Erhebung eines Sicherheitszuschlages auf die für Dieselruß geltenden Grenzwerte.

${ }_{245}$ Koch in: Hoffmann-Riem/Schneider (Fn. 65), 273, 274; Schulze-Fielitz ebd., 291, $303 \mathrm{f}$.
} 
Maßstabsbildend wirken ferner Über- und Untermaßverbot. Als Abwägungsregeln unter Ungewissheit ${ }^{246}$ sind sie auf die Erweiterung von Handlungsoptionen zur Realisierung verfassungsrechtlicher Schutzziele gerichtet ${ }^{247}$ und teilweise als Vertretbarkeitsklauseln kodifiziert. ${ }^{248}$ Insoweit ist in die Abwägung einzustellen, mit welcher Effektivität und zu welchem Preis eine Risikominimierung, ein Risikoausschluss oder kontrollierte Lerneffekte erreichbar erscheinen. ${ }^{249}$ Dabei ist die auch soziale Verteilung des Risikos zu bewerten und die Existenz von Substitutionsprodukten zu berücksichtigen. Bei hoher Abschätzungsunsicherheit wird sich die Abwägung nach dem Muster der einstweiligen Anordnung im Verfassungsprozess $^{250}$ durch Vergleich der Irrtumskosten der in Betracht kommenden Entscheidungsalternativen vollziehen. ${ }^{251}$ Ein Einschreiten kann danach angezeigt, sein, wenn das Zuwarten mit einem hohen Risiko des Fehlschlags späterer Abwehrbemühungen verbunden ist. ${ }^{252}$ Die insoweit zu treffenden Wertungen haben politisch-gestaltenden Charakter und sind rechtlich vornehmlich prozedural vorgeprägt. Das stellt ihre gerichtliche Überprüfung - auch im Rahmen einer gebundenen Entscheidung ${ }^{253}$ -

246 Dazu Köck (Fn. 39), 260; ferner Kastendieck(Fn. 99), 112ff., der bei seinen weiteren Überlegungen aber übersieht, dass in Fällen der Ungewissheit gerade auch ungewiss sein kann, mit welchen Schadensmöglichkeiten zu rechnen ist.

247 Ladeur (Fn. 129), 252; vgl. auch bereits oben III.2.c).

$248 \mathrm{Vgl}$. bereits oben II.3.

249 Ladeur (Fn. 129), 249; zu dieser „Kosten-Wirksamkeitsanalyse“ auch C. Calliess (Fn. 53), 54; sie muss freilich immer auch die jeweiligen Abschätzungsunsicherheiten berücksichtigen. Diese können geringer zu gewichten sein, wenn sich der Risikoverursacher zu projektbegleitender Risikoforschung verpflichtet.

250 Dazu etwa Graßhof in: Maunz u.a (Hrsg.) BVerfGG, $\$ 32$ Rn. $87 \mathrm{ff}$.

251 Ladeur (Fn. 129), 247; Köck (Fn. 39), 270; Berg (Fn. 49), 298; die Abwägung der Irrtumskosten ist vor allem dann geeignet, wenn die Art der fraglichen Schadenspotentiale feststeht, die Eintrittswahrscheinlichkeit aber nicht bestimmt werden kann; dabei stehen sich regelmäßig verschiedene Risiken und Chancen mit unterschiedlichen $\mathrm{Ab}$ schätzungsungewissheiten gegenüber, weshalb ein schlichtes Risikominimierungsgebot nicht behilflich wäre; zu diesem Murswiek, Möglichkeiten und Probleme bei der Verfolgung und Sicherung nationaler und EG-weiter Umweltschutzziele im Rahmen der europäischen Normung: Einflussmöglichkeiten der nationalen Politik auf die Ausgestaltung der rechtlichen Rahmenbedingungen von Normungsprozessen auf der Ebene der Europäischen Union - Rechtsgutachten im Auftrag des Deutschen Bundestages, 1995, www.jura.uni-freiburg.de/ioeffr3/papers/papers.htm, 81 .

252 Richter Gentechnologie als Regelungsgegenstand des technischen Sicherheitsrechts, 1989, 52; Kloepfer in: Korff/Beck/Mikat, Lexikon der Bioethik, Bd. III, 2000, 210, 211.

253 S. etwa Trute (Fn. 46), 66; auf die Einräumung eines Beurteilungsspielraums verweisen etwa $\$ \S 13,20$ b Abs. 19 . BImSchV, dazu Ohms UPR 2001, 87, $89 \mathrm{f}$. 
unter „Funktionsvorbehalt“. ${ }^{254}$ So geht auch der VGH Baden-Württemberg bei seiner Entscheidung zur Nanotechnologie von einem ungeschriebenen Beurteilungsspielraum im Rahmen der Vorsorge aus..$^{255}$

\section{(ee) Die Dynamisierung der Regulierung}

Noch stärker als bei der Entscheidungsfindung kommt es bei der Umsetzung konkreter Vorsorgeziele zu einer „Verantwortungsteilung“ zwischen öffentlicher Steuerung und privatwirtschaftlicher Selbstregulierung. ${ }^{256}$ Auf geeignete Mittel einer innovationsfördernden Risikosteuerung ist oben bereits hingewiesen worden. ${ }^{257}$ Heute kommen die direkten Steuerungsmittel des Ordnungsrechts ${ }^{258}$ meist neben den indirekten Steuerungsinstrumenten des Organisations-, Informations-, Abgabenund Haftungsrechts zum Einsatz. ${ }^{259}$ Belange des Vertrauensschutzes und der Investitionssicherheit werden dabei durch den sozialen Bezug der Betätigung in verhältnismäßiger Weise relativiert, so dass Genehmigungen im Bereich der Risikoverwaltung vielfach befristet erteilt werden, ${ }^{260}$ unter Änderungsvorbehalt stehen ${ }^{261}$ oder dem Betreiber die selbständige Aktualisierung seiner Sicherheitstechnik auferlegen. ${ }^{262}$

254 Dazu BVerwGE 106, 115, 122; 104, 36, 51; 85, 368, 379; 78, 177, 180; VGH BW VBIBW 2002, 436, 440; OVG Berlin, NVwZ 1995, 1023, 1024f.; OVG Berlin, NVwZ 1999, 96, 99; Kahl(Fn. 54), 1118; ferner Ladeurin: Hoffmann-Riem/Schmidt-ABmann (Fn. 47), 111, $126 \mathrm{ff.}$; Hoffinann-Riem ebd., 9, 24ff.; Preuß in: Grimm (Fn. 47), 542; Breuer NuR 1994, 157, 162; a. A. zum Pflanzenschutzrecht BVerwGE 81, 12, 17; zum gestaltenden Charakter der Risikoentscheidung und den sich daraus ergebenden Grenzen der gerichtlichen Kontrolle allgemein Di Fabio (Fn. 62), 270ff.; Lange/Karthaus in: Lange (Hrsg.) Gesamtverantwortung statt Verantwortungsparzellierung im Umweltrecht, 1997, $15 \mathrm{ff}$.; Scherzberg (Fn. 47), $171 \mathrm{ff}$.

255 VGH BW VBIBW 2001, 436, $439 \mathrm{f}$.

256 Dazu Schulze-Fielitz (Fn. 3), 482ff.; Di Fabio VVDStRL 56 (1997), 235, $242 \mathrm{ff}$.

257 Dazu oben III.2.e); vgl. auch Kahl (Fn. 54), 1113.

${ }^{258} \mathrm{Zu}$ dessen vermehrtem Einsatz im Rahmen europarechtlicher Risikosteuerung jüngst Köck in: Rengeling (Fn. 160), 33 f.

${ }^{259}$ Dazu aus Sicht des Zivilrechts Damm in Bora (Fn. 26), 93, 121; aus Sicht des öffentlichen Rechts Schmidt-Aßmann (Fn. 49), 1/37.

$260 \mathrm{Vgl} . \S 7$ Abs. 1 WHG, $\S 16$ Abs. 1 S. 1 PflSchG, $\$ 31$ Abs. 1 Nr. 3 AMG; $\S 17$ Abs. 1 S. 3 AtG.

$261 \S 17$ Abs. 3 AtG, $§ 26$ Abs. 1 Nr. 1 - 3 GenTG, $\S 30$ AMG, $\$ 16$ a Abs. 2 PflSchG, $\$ 5$ Abs. 1 WHG, Art. 13 Abs. 1 RL 96/61; Schmehl Genehmigungen unter Änderungsvorbehalt zwischen Stabilität und Flexibilisierung, 1998, $149 \mathrm{ff}$.

$262 \S 5$ Abs. 1 Nr. 2 BImSchG, $§ 6$ Abs. 2 GenTG; $\$ 15$ a Abs. 1 PflSchG; daran fehlt es aber etwa im Bereich der Novel-Food Verordnung, kritisch dazu Meier, Risikosteuerung im Lebensmittel- und Gentechnikrecht, 2000, 144; Wahl/Gro $\beta$ DVBI. 1998, 2, 10; zum Ganzen auch Wahl/Hermes/Sach in: Wahl (Fn. 23), 217, 233 ff.; Trute (Fn. 46), 74f.; Sach Genehmigung als Schutzschild?, 1994, 112ff., 148f.; eine Pflicht zur Fortentwicklung des 


\section{b) Die Risikoprävention}

\section{(aa) Das Regelungsmodell}

Präventive Risikosteuerung, der zweite Aufgabenkreis der Risikoverwaltung, ist Zukunftsvorsorge mit „offenem Suchhorizont" ${ }^{\text {"263 }}$ und zielt auf die Minimierung des (noch) unspezifischen Risikopotentials umweltund gesundheitsrelevanter Aktivitäten. ${ }^{264}$ Mit ihr reagiert das Recht auf den zuletzt am Stromnetz bekannter Industriestaaten dokumentierbaren Befund, dass in eng gekoppelten technischen Systemen prinzipiell mit unvorhersehbaren negativen Synergismen gerechnet werden muss, ${ }^{265}$ und auf die allgemeine umweltpolitische Erfahrung, dass nachteilige Folgen dauerhafter zivilisatorischer Umwelteinwirkungen heute nach ,praktischer Vernunft" kaum jemals ausgeschlossen werden können. ${ }^{266}$

\section{(bb) Die Instrumente}

\section{(I) Das ökologische Erforderlichkeitsgebot}

Geeignetes Instrument zur Risikoprävention ist die generelle Verpflichtung der Umweltnutzer auf eine Minimierung von Umwelteinwirkungen und Ressourcenverbrauch, ein "ökologisches Erforderlichkeitsgebot", das jede nicht nachweislich unschädliche Einwirkung auf Mensch und Umwelt als rechtfertigungsbedürftig hinterfragt. ${ }^{267}$ Es belässt die Aus-

\footnotetext{
jeweiligen Standes der Wissenschaft durch Risikoforschung kennt das derzeitige Risikoverwaltungsrecht dagegen kaum; vgl. aber $\$ 28$ Abs. 3a AMG, dazu Köck in: Schnapp (Hrsg.) Wie sicher sind unsere Arzneimittel? (im Erscheinen), MS, 14f.; das wird zu Recht kritisiert, ist es doch die wissensgenerierende Funktion der Risikosteuerung, die sie „über die Zeit“" an Übermaß- und Untermaßverbot zurückbindet; kritisch etwa Murswiek (Fn. 126), 218.

${ }^{263}$ Gill (Fn. 41), 30 f.

${ }^{264}$ Zur Notwendigkeit präventiver Entwicklungssteuerung Grimm (Fn. 70), 418; Dierkes/Canzler in: Hoffmann-Riem/Schneider (Fn. 65), 63, 76ff.; Hoffmann-Riem/Schneider (Fn. 65), $407 \mathrm{ff}$;; Minsch/Elberle/Meier/Schneidewind Mut zum ökologischen Umbau, 1996, 121 ff.; Wolf in: Roßnagel/Neuser (Fn. 21), 57, $66 \mathrm{ff}$.

${ }^{265}$ Perrow Normale Katastrophen, 1989, $16 \mathrm{ff}$., $129 \mathrm{ff}$; ders. Journal für Sozialforschung 32 (1992), 61, 62 ff, $66 \mathrm{ff}$;; deshalb bietet auch die probabilistische Risikoanalyse mit ihrer Anknüpfung an die Versagenswahrscheinlichkeit einzelner Systemkomponenten keine „sichere“ Berechnungsgrundlage, vgl. etwa Banse in: ders. (Fn. 2), 64; Seiler (Fn. 111), 213ff.; zu optimistisch daher Breuer (Fn. 254), $162 \mathrm{ff}$.; s. auch Bergmann in: Banse (Fn. 2), 83, $88 \mathrm{ff}$. mit einem Bericht über Untersuchungen zur systematischen Überforderung des Menschen bei der Überwachung und Regelung dynamischer Systeme.

266 Rehbinder (Fn. 50), 04/50.

${ }^{267}$ Zur Diskussion darüber im Kontext der Vorsorge instruktiv Roßnagel in: Koch/ Scheuing (Fn. 243) § 5 BImSchG Rn. 527 ff. mwN.
} 
wahl von Unternehmenszielen, Herstellungsverfahren und Produktgestaltung beim Betreiber, sichert aber, durch die Verpflichtung auf eine zumutbare, ökologisch verträgliche Alternative, ${ }^{268}$ die generelle Minimierung des Risikos. ${ }^{269}$ Das ökologische Erforderlichkeitsgebot schützt die künftigen Lebensgrundlagen dadurch, dass es die Risiken der Unterschätzung heutiger Umwelteinwirkungen reduziert und gründet mithin in der „Auftragsdimension“ des Art. 20a GG. ${ }^{270}$

Ansätze für eine Reduzierung der Exposition oder Ressourcennutzung unabhängig von vermuteten Wirkungsgrenzen finden sich in $\S 1 \mathrm{a}$ Abs. 1 S. 2 WHG, wonach Gewässer so zu bewirtschaften sind, dass jede vermeidbare Beeinträchtigung ihrer ökologischen Funktion unterbleibt, ${ }^{271}$ in $\S \S 18 \mathrm{f}$. BNatSchG, wonach vermeidbare Beeinträchtigungen von Natur und Landschaft zu unterlassen sind, im Gebot sparsamer Energieverwendung des $\S 5$ Abs. 1 Nr. 4 BImSchG ${ }^{272}$ sowie im Strahlenschutzrecht, Bodenrecht und Energiewirtschaftsrecht. ${ }^{273}$

\section{(2) Das Stoff- und Energiestrommanagement}

Seine Fortsetzung findet die präventive Risikosteuerung in der Einwirkung auf ressourcen- und risikorelevante Verhaltensmuster im Umgang mit Energie, Stoffen, Produkten, Raum und Verkehr. ${ }^{274}$ Beispiele sind die Verteuerung des Ressourcenverbrauchs durch Umweltabgaben, ${ }^{275}$ die Einführung einer verbindlichen Umweltbelastungsanalyse für neue Pro-

\footnotetext{
268 So zum Pflanzenschutzrecht BVerwGE 81, 12, 17; Köck (Fn. 39), 195ff.; vgl. auch $\$ 16$ Abs. 2 S. 1 und $\$ 36$ Abs. 2 S. 1 GefStoff V.

${ }^{269}$ Präziser am Beispiel des Gebots effizienter Energieverwendung Koch/Wieneke (Fn. 144), 1090.

${ }^{270} \mathrm{Zu}$ dieser Lübbe-Wolff(Fn. 48), 66f.

271 Dazu Rehbinder (Fn. 50), 04/37.

272 Dazu Britz in: Lange (Hrsg.) Nachhaltigkeit im Recht, 2003, 145, 165 ff.; Koch/Wieneke (Fn. 144), $1089 \mathrm{ff}$.

${ }^{273}$ Vgl. \$ 6 Abs. 1, 2 StrSchV, dazu Bender/Sparwasser/Engel Umweltrecht, 4. Aufl. 2000, Kap. 9 Rn. $243 ; \S 7$ S. 3 BBodSchG; $\$ 1$ Abs. 1, $\$ 2$ Abs. 1, 33 Abs. 1 EnEG; s. auch Ziff. 5. 2. 7. TA Luft 2002 für schwer abbaubare und leicht anreicherbare organische Stoffe und $\S \S 1,4$ Abs. 1 Nr. $1 \mathrm{KrW}-/ \mathrm{AbfG}$; zum künftigen EG-Chemikalienrecht mit seinem Ziel der Substitution gefährlicher durch weniger gefährlicher Stoffe Köck in: Rengeling (Fn. 160), 21 f.; C. Calliess (Fn. 53), $57 \mathrm{ff}$.

274 Dazu allgemein Beaucamp (Fn. 148), $327 \mathrm{ff}$; Lübbe-Wolff Modernisierung des Umweltordnungsrechts, 1996, 115ff.; Jörissen (Fn. 171), $7 \mathrm{ff} . ;$ Wolf in: Roßnagel/Neuser (Fn. 21), 57, 72ff.

275 Dazu Steinberg (Fn. 13), 157 ff.; Kirchgässer in: Grimm (Fn. 47), 453, 448ff.; LübbeWolff(Fn. 274), 105ff.; zur Weiterentwicklung der derzeitigen Öko-Steuer vgl. Sachverständigenrat (Fn. 22), Tz. $461 \mathrm{ff}$.
} 
dukte, ${ }^{276}$ die Verpflichtung zur abfallarmen Produktgestaltung ${ }^{277}$ und die Zusammenfassung dieser und anderer Instrument zu einer integrierten Produktpolitik. ${ }^{278}$ Diese zielt auf eine Stoffstromsteuerung, die zur Reduktion von Stoffverlusten, zur Verlangsamung von Stoffströmen und dadurch zur generellen Minderung der Risikofaktoren zivilisatorischer Umweltnutzung beiträgt. 279

\section{(3) Die Risikohaftung}

Auch das Haftungsrecht wirkt präventiv auf das Produktdesign ein. ${ }^{280}$ Vor allem Beispiele aus den USA zeigen allerdings, dass hohe Haftungsrisiken auch zu erheblichen Wettbewerbsbelastungen beitragen und innovationshemmend wirken. ${ }^{281}$ Risikominimierende Funktion kann das Haftungsrecht ohnehin nur übernehmen, soweit der Eintritt eines Haftungstatbestandes bei der unternehmerischen Entscheidung antizipiert und vermieden werden kann. ${ }^{282}$ Deshalb liegt die Begrenzung der Haftung auf solche Risikoquellen nahe, die bei zumutbarer Risikoforschung

276 Dazu Rehbinder/Schmihing Ökobilanzen als Instrumente des Umweltschutzes, 2000, 98 ff.; ferner Umweltbundesamt (Hrsg.) UGB-AT, 1992, UGB-BT, 1994, $\$ \$ 10$ Abs. 1 , 464 ProfE-UGB; Bundesministerium für Umwelt, Naturschutz und Reaktorsischerheit (Hrsg.) Umweltgesetzbuch - Entwurf der unabhängigen Sachverständigenkommission, $\S 191$ UGB-KomE.

277 Dazu $\$ 22$ Abs. 1 S. 2 KrW-/AbfG und die Grundsätze der Abfallvermeidung und der abfallarmen Produktgestaltung gem. $\S 4$ Abs. 1 Nr. 1, Abs. 2 KrW-/AbfG, die auch auf den Verbrauch von Materialien für die Produktgestaltung zurückwirken; dazu Beaucamp (Fn. 148), $364 \mathrm{ff}$.

278 Grünbuch der Europäischen Kommission zur Integrierten Produktpolitik, KOM (2001), 68 endgültig; Mitteilung der Kommission an den Rat und das europäische Parlament, Integrierte Produktepolitik KOM (2003) 302 endgültig; dazu Sachverständigenrat (Fn. 22), Tz. 64* ff., 379 ff.; Jörissen (Fn. 171), 7 f.

279 Dazu Beaucamp (Fn. 148), 327ff., 330; ferner die Beiträge in: Führ (Hrsg.) Stoffstromsteuerung durch Produktregulierung, 2000; $\mathrm{zu}$ den damit verbundenen Erhebungs- und Abwägungsproblemen etwa Lübbe-Wolff(Fn. 274), 119f.; Brandt/Rückeisen Konzeption für ein Stoffstromrecht, 2000; Zundel/Bunke/Schramm/Steinfeldt ZfU 1998, $317 \mathrm{ff}$; Zwischenbericht Enquete-Kommission „Schutz des Menschen und der Umwelt“, BT-Drs. 12/5812, $18 \mathrm{ff}$., $133 \mathrm{ff}$. sowie die Beiträge in Brickwedde (Hrsg.) Stoffstrommanagement - Herausforderungen für eine nachhaltige Entwicklung, 1999.

280 Dazu Damm in Bora (Fn. 26), 93, 117; Salje in: Schulte (Fn. 3), 73, 86f.; Endres in: ders./Rehbinder/Schwarze, Haftung und Versicherung für Umweltschäden aus ökonomischer und juristischer Sicht, 1992, 1, 4 ff.; Rehbinder ebd., 34, 54ff.; skeptisch etwa Marburger AcP 192 (1992), 1 ff.; Hapke (Fn. 208), 55 ff., dazu auch bereits oben IV.2.b).

281 Viscuse/Moore Journal of Political Economy 101 (1993), $161 \mathrm{ff}$.; Morrow (Fn. 44), $26 \mathrm{f}$; d der Mittelweg dürfte in einer angemessenen Bemessung von Haftungshöchstgrenzen liegen vgl. Hapke/Japp (Fn. 105), 157f.

${ }^{282}$ Hapke (Fn. 208), $56 \mathrm{ff}$. 
des Betreibers hätten erkannt und ausgeschaltet werden können. ${ }^{283}$ In diesem Sinne machen das ProdHaftG ${ }^{284}$ und der Richtlinienentwurf zur Umwelthaftung 285 den vorgesehenen Ausgleich für Schäden aus Entwicklungsrisiken vom Verschulden des Herstellers abhängig. ${ }^{286} \mathrm{Mehr}$ noch als dessen Verurteilung wirkt präventiv allerdings die mit dem Haftungsprozess verbundene negative Publizität.

\section{(4) Die Risikokommunikation}

Das verweist auf ein letztes Mittel präventiver Risikosteuerung: die Information. Risikobewusste Entscheidungen bedürfen einer verlässlichen informationellen Grundlage, die sich marktförmig nicht sicher herstellen lässt. 287 Zur Gewährleistung einer „ökologischen Markttransparenz“288 eignen sich neben punktuellem behördlichen Eigenhandeln in Warnungen und Empfehlungen ${ }^{289}$ deshalb vor allem Anreize und Pflichten zur unternehmerischen Verbraucheraufklärung, etwa durch das Angebot der Nutzung zertifizierter Umweltzeichen oder durch produktbezogene Informations- und Kennzeichnungspflichten. ${ }^{290}$

Das Wissen um Ressourcenverbrauch und Abfallmengen wirkt freilich motivatorisch nur, wenn sich daran für die Verbraucher ein erkennbarer

283 Näher Kötz/Wagner (Fn. 143), Rn. 131 ff.; Endres (Fn. 280), 8, 29; Ekardt/Manger/ Neuser/Pottschmidt/Roßnagel/Rust (Fn. 76), $170 \mathrm{ff}$.

284 Vgl. $\$ 1$ Abs. 2 Nr. 5 ProdHaftG; dazu Vieweg in: Schulte (Fn. 3), 329, 365 ff.

285 Vgl. Art. 9 Abs. 1 c, d, Abs. 2 Entwurf der Richtlinie zur Umwelthaftung, KOM (2002) 17 endgültig.

${ }^{286}$ Zum Streit um die Angemessenheit der Gefährdungshaftung für Entwicklungsrisiken Niklisch in: Jayne/Laufs/Misera/Reichart/Serick (Hrsg.) FS Niederländer, 1991, 341, $342 \mathrm{ff}$.; Hapke (Fn. 208), 75 ff.; offenbar werden Entwicklungsrisiken heute von der Industrie für so unwahrscheinlich gehalten, dass Vorkehrungen dagegen als unvertretbar kostenintensiv gelten, vgl. Hapke/Japp (Fn. 105), 74f.

${ }^{287}$ Krücken in: Hiller/Krücken (Fn. 26), 116, 140 f.; Kahl (Fn. 54), 1114 ff.

288 Sachverständigenrat (Fn. 22), Tz.17* ff., $75 \mathrm{ff}$.

289 Dazu BVerfGE 105, $252 \mathrm{ff}$. (Glykol) und $279 \mathrm{ff}$. (Osho); dazu zu Recht kritisch Huber JZ 2003, 290ff.; Murswiek NVwZ 2003, 1 ff.; s. ferner Stoll (Fn. 50), 287 ff.; Erichsen Zur Umsetzung der Richtlinie des Rates über den freien Zugang zu Informationen über die Umwelt, 1992, $124 \mathrm{ff}$.

290 Allgemein Sachverständigenrat (Fn. 22), Tz. 17* ff., 75 ff., 381; zu den zivilrechtlichen Informationspflichten Möllers Rechtsgüterschutz im Umwelt- und Haftungsrecht, 1996, 291 ff., 370ff., 385f.; zu Warnhinweisen auf Zigarettenpackungen BVerfG NJW 1997, 2871, 2872; zum EU-Umweltzeichen Sobczak (Fn. 171), 126ff.; zur EG-Energieetikettierung RL 92/75/EWG v. 22.9. 1992 über die Angabe des Verbrauchs an Energie und anderen Ressourcen durch Haushaltsgeräte mittels einheitlicher Etiketten und Produktinformationen, ABI. L 297/16. 
Nutzen knüpft. ${ }^{291}$ Diesen darzustellen ist eine der Aufgaben der Risikokommunikation. ${ }^{292}$ Sie vollzieht sich nicht zuletzt durch die politisch-administrative Entwicklung und Verbreitung von Leitbildern. ${ }^{293}$

Dabei handelt es sich um langfristige Ziele und Visionen der Risikopolitik wie die „Nachhaltigkeit der Ressourcennutzung“, ${ }^{294}$ die „grüne Landwirtschaft“ oder das „3-Liter-Auto“, die weitgehend konsentierte kollektive Interessen in einer öffentlich wahrnehmbaren Weise verkörpern und im Rahmen der Regelorientierung menschlichen Verhaltens maßstabbildend auf die individuelle und organisationelle Selbststeuerung wirken. ${ }^{295}$ Sie verkörpern Ideen, aus denen die Inspiration zu gemeinschaftsverträglichen Innovationen erwächst ${ }^{296}$ und die Bereitschaft, die Risiken dieser Innovationen zu akzeptieren.

\section{Ausblick: Rechtliche Ungewissheitsbewältigung und ihre Rückwirkungen auf das Recht}

Der Umgang mit dem Risiko ist auch für das Recht selbst riskant. Die Ungewissheit seines Regelungsgegenstandes wirkt in funktionaler, methodischer, theoretischer, dogmatischer und vollzugspraktischer Hinsicht auf das Recht zurück.

Funktional muss sich das Recht, statt Erwartungssicherheit zu vermitteln, ${ }^{297}$ um das Offenhalten von Handlungsoptionen und um Lernfähigkeit bemühen. Es kann der gesellschaftlichen Regelorientierung vielfach

\footnotetext{
${ }^{291} \mathrm{Vgl}$. Minsch/Eberle/Meier/Schneidewind (Fn. 264), 161 ff., nach deren Analyse vor allem die unvollkommene Wahrnehmung des Nutzens risikominimierender Innovationen deren Durchsetzung behindert.

${ }^{292}$ Dazu die Vorschläge der Risikokommission, Abschlussbericht (Fn. 52), 53ff., 57; ferner Kloepfer/Wimmer UPR 1993, 409, 414; Sachverständigenrat, Sondergutachten (Fn. 52), Tz. 20* ff., $112 \mathrm{ff}$.

${ }^{293}$ Dazu Dierkes/Canzler in: Hoffmann-Riem/Schneider (Fn. 65), 66ff.; Dierkes/Hoffmann/Marz Leitbild und Technik, 1992, $15 \mathrm{ff}$., $153 \mathrm{ff}$.; Karstens in: Eifert/Hoffmann-Riem (Fn. 138), 50ff.; Baer in: Schmidt-Aßmann/Hoffmann-Riem, Methoden der Verwaltungsrechtswissenschaft (im Erscheinen), MS, $13 \mathrm{ff}$.

${ }^{294}$ Dazu Kahl in: Bauer/Czybulka/Kahl/Voßkuhle (Hrsg.) Umwelt, Wirtschaft und Recht, 2002, 111, 148.

${ }^{295}$ Sie setzen dazu eine langfristig angelegte und richtungsstabil umgesetzte Risikopolitik voraus, dazu Umweltbundesamt (Fn. 139), 356ff.

296 Dazu bereits oben III.2.c).

${ }^{297} \mathrm{Zu}$ dieser Aufgabe des Rechts etwa Luhmann Das Recht der Gesellschaft, 1993, 131 f.; Hiller Der Zeitkonflikt in der Risikogesellschaft, 1993, 60f.; Nocke JSRpol 1990, $123,133 \mathrm{f}$.
} 
keine konkreten Prämissen mehr bieten, sondern muss sich auf mehrfach anschlussfähige Vorgaben beschränken. Insoweit überträgt es Mitverantwortung an seine Adressaten und übernimmt eine influenzierende und edukatorische Funktion. ${ }^{298}$

Methodisch wird das Recht unter Ungewissheit reflexiv. Soweit die Folgen einer (Fehl-)Entscheidung vom Gesetzgeber nicht bereits abschliessend verarbeitet sind, bedarf es einer Entscheidungsfolgenabschätzung durch die Verwaltung. Wenn diese dabei über temporäre Maßnahmen anhand langfristiger Sicherheitskonzepte entscheidet, kommt der Kohärenz des Entscheidungszusammenhangs maßgebende Bedeutung zu. 299

(Steuerungs-)Theoretisch muss das Recht das Risikowissen, das Handlungspotential und die Kontextbindungen der marktförmigen, professionellen, normungstechnischen, wissenschaftlichen und personalen Selbststeuerung integrieren. Es wird damit responsiv und eröffnet den Raum für die Entfaltung von Innovationen.

Dogmatisch müssen die auf stabile Zurechnungen angewiesenen Institute des Übermaß- und Untermaßverbots in eine Abwägungsregel unter Ungewissheit transformiert werden. Zugleich wandelt sich der Gesetzesvorbehalt von einem Prinzip begrenzter Eingriffsermächtigung zu einem Gebot normativer Qualitätssicherung durch inhaltliche, prozedurale und organisationelle Steuerung. ${ }^{300}$ Damit gibt das Recht die Forderung nach „fest begrenzten Befugnissen der Verwaltung ${ }^{\text {“301 }}$ auf. ${ }^{302}$ Zugleich wird die gerichtliche Kontrolle der Risikoentscheidung auch als „gebundene Entscheidung" funktionellrechtlich relativiert.

Dadurch vermindert sich bei Ungewissheit die Entlastungsfunktion des Rechts und steigert sich der politische Bewertungs- und Gestaltungsbedarf. Das erhöht die Anforderungen an die Beurteilungskompetenz und die Verantwortungsfähigkeit der öffentlichen und privaten Entscheidungsträger. ${ }^{303}$

Im Recht wird die Risikogesellschaft damit trotz aller Reduktion, Prävention und Reflexion ,auf der Suche nach der verlorenen Sicherheit“"304

298 Zur edukativen Funktion des Rechts auch Scherzberg (Fn. 80), 43 f.

${ }^{299}$ Zur Methodendiskussion insoweit Röhl Allgemeine Rechtslehre, 2. Aufl. 2001, $605 \mathrm{ff}$;; Hoffmann-Riem ZfRSoz 2001, 3, 13; für Risikoentscheidungen etwa Di Fabio (Fn. 48), 573.

300 Dazu Di Fabio (Fn. 62), 465f.; zurückhaltend Wahl/Appel (Fn. 23), 115 f.

301 Anschütz in: Meyer, Lehrbuch des deutschen Staatsrechts, 6. Aufl. 1905, 27.

302 Scherzberg (Fn. 47), 157.

303 Dazu bereits Scherzberg in: Engel/Halfmann/Schulte (Fn. 14), 113, $143 \mathrm{f}$.

304 Fischer (Hrsg.) Auf der Suche nach der verlorenen Sicherheit, 1991. 
nicht fündig. ${ }^{305}$ Das Recht kann nur ihren Umgang mit der Ungewissheit strukturieren. Weitergehende Sicherheit findet die Gesellschaft nur in sich selbst. Denn Risiko ist ein Konstrukt. Man muss es nicht als seinen Beobachtungsstandpunkt wählen.

305 Scherzberg in: Engel/Halfmann/Schulte (Fn. 14), 113, $142 \mathrm{f}$. 
Leitsätze des 1. Berichterstatters über:

\section{Risikosteuerung durch Verwaltungsrecht: Ermöglichung oder Begrenzung von Innovationen}

\section{Der Ausgangsbefund: das Phänomen des Risikos}

1. Die moderne Gesellschaft steigert mit ihrem wissens- und technikbasierten Handlungspotential zugleich Sicherheit und Ungewissheit. Das Konzept von Risiko und Chance gibt dieser Entwicklung Ausdruck. Es soll Entscheidungsrationalität unter Ungewissheitsbedingungen herstellen.

2. Dabei tritt das Risiko dem Recht in zwei Erscheinungsformen entgegen: als Schadenspotential in der von ihm regulierten Umwelt und als mögliche Irrtumskosten der rechtlichen Regulierung selbst.

\section{Begriff und Theorie des Risikos}

3. Für die Zwecke rechtlicher Risikoverarbeitung sind deshalb zwei Risikobegriffe erforderlich. Das Risiko erster Ordnung bezeichnet den Erwartungswert eines Schadens, das Risiko zweiter Ordnung die Folgen seiner Fehleinschätzung.

4. Zur Bewältigung moderner Technikfolgen muss das Recht von einer erfahrungsbasierten auf eine ungewissheitsbasierte Strategie normativer $\mathrm{Zu}$ kunftsbewältigung übergehen und im Rahmen der Vorsorge „alle vertretbaren Erkenntnisse“ und auch „nur theoretische Überlegungen“ in die Risikokalkulation einbeziehen. Je weiter es sich damit in die Ungewissheit hinein ausweitet, desto größer wird die Möglichkeit der Fehleinschätzung und Fehlsteuerung und damit das Risiko zweiter Ordnung.

5. Um ihm zu begegnen, wird rechtliche Risikosteuerung reflexiv. Neben den vermuteten Auswirkungen einer Technikanwendung berücksichtigt sie auch die Ungewissheit und die möglichen Irrtumskosten der rechtlichen Regulierung. Das Wissen um das Nichtwissen kann dabei den Übergang auf Instrumente der Risikosteuerung nahe legen, die auf der Grundlage geringerer Erkenntnissicherheit Erfolg versprechen. Hohe Irrtumskosten werden regelmäßig die Grenze der Vorsorge unter Ungewissheit indizieren: Restrisiko ist dasjenige Risiko, bei dem die Risiken der Risikosteuerung überwiegen. 


\section{Reflexive Risikosteuerung: Theoretische Grundlagen und Wirkungsbezüge}

6. Wird das Recht reflexiv, wird die Rechtswissenschaft zur Steuerungswissenschaft. Als solche macht sie erkennbar, dass das Recht unter bestimmten Bedingungen Wirkungen erzeugt, die der Absicht seiner Setzung zuwiderlaufen.

7. Der Ausübung staatlicher Zwangsgewalt sind gerade im Bereich der Ungewissheit neben rechtsstaatlichen auch funktionale Grenzen gesetzt. Risikosteuerung ist deshalb nicht als zwangsweise Fremdbestimmung, sondern als kommunikative Einwirkung auf die Selbstbestimmung ihrer Adressaten zu konzipieren.

8. Sie kann sich dabei auf die Erkenntnisse der Verhaltenswissenschaften stützen. Danach steht neben der Nutzenmaximierung die Regelorientierung als weitere Form menschlicher Entscheidungsrationalität. Daran kann das Recht anknüpfen. Es muss dazu allerdings die Abhängigkeit der Regelungsadressaten von sozialer Akzeptanz, wirtschafilichem Erfolg und langfristigen Planungshorizonten in Rechnung stellen. Erfolgreiche Risikosteuerung ist deshalb „responsive Regulierung“.

9. Risikosteuerung beginnt mit der Risikokonstitution. Diese darf das Recht nicht in vollem Umfang an die Risikoforschung delegieren, sondern muss die naturwissenschafiliche Realanalyse mit ihren Konventionen an rechtlich spezifizierte Schutzziele zurückbinden und den Umgang mit der Abschätzungsunsicherheit standardisieren.

10. In die Risikobewertung und die Entscheidung über die Akzeptabilität des Risikos fließen die von der Psychologie aufgedeckten individuellen und kollektiven Muster der Verarbeitung von Ungewissheit ein. Überdies bedarf es eines Vergleichs der Folgen der in Betracht kommenden Maßnahmen der Risikosteuerung. Dieser Risikovergleich verkörpert die Rationalität der Risikoabwägung und in seiner Komplexität auch deren Grenzen.

11. Risikosteuerung darf weder Ungewissheit verdrängen noch Erfahrungsgewinn vermeiden, sondern muss darauf zielen, die gesellschafiliche Handlungsfähigkeit im Umgang mit dem Risiko insgesamt zu erhöhen.

12. Sie ist im Kontext von Innovation deshalb so zu konzipieren, dass sich Regulierung und technischer Innovationsprozess wechselseitig stärken. Dazu muss das Recht langfristige Sicherheitserwartungen an Wirtschaft und Technik formulieren und die Verfahren und Instrumente der konkreten Regulierung so ausgestalten, dass sie neues Wissen flexibel integrieren.

13. Sie kann damit Innovationen anregen und in eine gewünschte Richtung lenken, wenn der rechtlich erzeugte Innovationsdruck in geeigneter Weise auf die Innovationsfähigkeit und die verfügbaren Ressourcen der Akteure abgestimmt ist. Das gelingt nach den Vorschlägen der Innovationsökonomie durch einen Mix aus ökonomischen und ordnungsrechtlichen Instrumenten. 
Ökonomische Vermeidungsanreize erlauben eine flexible Selbststeuerung. Ordnungsrechtliche Vorgaben geben langfristige Orientierung und können durch angemessen anspruchvolle Zulassungserfordernisse zur Bildung eines "führenden Marktes" beitragen.

\section{Das Recht der reflexiven Risikosteuerung}

14. Weite Teile des Risikoverwaltungsrechts sind heute mittelbar oder unmittelbar gemeinschafisrechtlich geprägt. Kennzeichnend für die gemeinschafisrechtliche Vorsorge sind neben materiellen Vorgaben vor allem die Prozeduralisierung der Risikosteuerung, die Harmonisierung der technischen Normung, die zunehmende Übertragung von Koordinations- und Durchführungsbefugnissen an die Kommission und die Beteiligung europäischer Behörden an der Risikoabschätzung.

15. Das Grundgesetz verpflichtet zur Definition und Durchsetzung angemessener Einwirkungsgrenzen auf individuelle und kollektive Güter und zur Sicherung eines größtmöglichen Rahmens wirtschaftlicher Entfaltung. Die danach geforderte Vorsorge umfasst alle Fälle wissenschaftlich begründeter Besorgnis. Bei Ungewissheit führt der Verweis auf die Wissenschaft indes ins Leere. Deshalb ist der Staat bei der Eröffnung eines Risikos zur Gewährleistung weiterer Wissensgenerierung verpflichtet. Grundrechtliche Schutzpflichten gestatten die Begründung eines (Rest)Risikos nur, soweit und solange die Risiken der Risikoreduktion überwiegen.

16. Der Präventionsstaat, dem Art. 20 a GG die Verantwortung für die natürlichen Lebensgrundlagen überträgt, muss auch jene alltäglichen Beanspruchungen der Umweltgüter problematisieren, deren Summations- und Fernwirkungen die Regenerationsfähigkeit des Naturhaushalts erst in der weiteren Zukunft bedrohen. Ihm obliegt deshalb auch die "Zukunftsvorsorge" gegenüber heute noch unbekannten Eventualitäten.

17. Die grundrechtlichen Übermaß- und Untermaßverbote sind unter Ungewissheitsbedingungen kaum operabel. Ihre materiellen Unterschiede werden durch den funktionalen Einschätzungs- und Gestaltungsspielraum des Gesetzgebers für Prognosen und Wertentscheidungen überlagert. Für die „Wirkungstiefe" verfassungsrechtlicher Anforderungen ist es deshalb im Ergebnis unerheblich, ob eine Regelung das Risiko für ein Grundrechtsgut durch Eingriff erhöht oder durch Schutzgewährung mindert.

18. Bei seiner Mittelwahl ist der Gesetzgeber „weder auf marktferne ordnungsrechtliche noch auf marktkonforme ,ökonomische Instrumente beschränkt“. Rechtsgüterschutz lässt sich aber nicht auf finanzielle Kompensation reduzieren. Grundrechtliche Schutzaufträge sind deshalb durch die Begründung von Haftungs- und Versicherungspflichten allein nicht erfüllbar. 
19. Soweit die mangelnde Regelungsreife der Materie und der Zwang zur Dynamisierung der Vorsorge einer inhaltlichen Maßstabsbildung entgegenstehen, ist die Erreichung verfassungsrechtlicher Schutzziele prozedural zu gewährleisten. Dazu gehören die Standardisierung der bisher ungeregelten Verfahren der Risikoabschätzung sowie die Gewährleistung einer fortlaufenden Evaluation des Steuerungserfolgs.

20. Die Komplexität europarechtlicher Vorsorgeanforderungen, verfassungsrechtlicher Schutzpflichten und umweltstaatlicher Verantwortung lässt sich auf zwei Aufgabenbereiche des Risikoverwaltungsrechts zurückführen: Risikoreduktion bei hinreichend bekannten und Risikoprävention bei unbekannten oder nur vermuteten Risiken.

21. Zur Risikoreduktion wird in den Tatbeständen von Gefahrenabwehr und Vorsorge die Grenze der Belastbarkeit der betroffenen Güter definiert und über die Vermeidbarkeit ihrer Beeinträchtigung entschieden. Unter Ungewissheit lässt sich die Risikosteuerung dabei gesetzlich freilich nur in beschränktem Umfang determieren und wird die Maßstabsbildung regelmäßig auf wissensgenerierende administrative Verfahren verlagert.

22. Prozedurale Rationalität zielt insoweit darauf, im Verfahren der Normkonkretisierung und -umsetzung rechtliche, verwaltungspolitische, betriebliche, professionelle, wissenschafilich-technische und soziale Perspektiven in optimaler Weise zu entfalten. Sowohl bei der Anknüpfung an technische Standards als auch bei der Verwertung der Ergebnisse wissenschaftlicher Risikoabschätzung bedarf es dabei einer „steuernden Rezeption“. Soziale Risikokompetenz kann um so eher eingebunden werden, je mehr die Verfahren als Prozess sozialer Verständigung ausgestaltet sind und über die formalisierten Abläufe von Anhörung und Erörterung hinausgehende persönliche Interaktionen erlauben.

23. Fehlt es an einer ausdrücklichen gesetzlichen Beweislastverteilung, kann abhängig vom Schutzzweck der Norm zu berücksichtigen sein, dass hohe Anforderungen an die Beweisführung vor allem bei unbekannten Stoffen als negativer Anreiz für Risikoforschung und Innovation wirken. Die beiderseitige Wissensgenerierung wird gefördert, wenn dem Risikoverursacher auferlegt wird, diejenigen Schädlichkeitshypothesen zu falsifizieren, für die die Behörde begründete Anzeichen vorbringt.

24. Soll gesichert werden, dass rechtliche Risikosteuerung im Einzelfall „more good than harm “ bewirkt, kann dies vor dem Hintergrund der Lückenhaftigkeit des Risikowissens nicht quantitativ berechnet, sondern nur qualitativ entschieden werden. Vorsorgeziel und Gleichheitssatz verpflichten insoweit auf Kohärenz. Kohärenz verknüpft langfristige Zielbestimmung mit kurzfristiger Mittelwahl und verlangt dazu eine in Konzepten formalisierte oder in Einzelentscheidungen fortgebildete administrative „Risikopolitik“ Bei hoher Abschätzungsungewissheit wird sich die Abwägung im übrigen nach dem 
Muster der einstweiligen Anordnung im Verfassungsprozess durch Vergleich der Irrtumskosten der in Betracht kommenden Entscheidungsalternativen vollziehen.

25. Noch stärker als bei der Entscheidungsfindung kommt es bei der Umsetzung der Risikoentscheidung zu einer "Verantwortungsteilung" zwischen öffentlicher Steuerung und privatwirtschafticher Selbstregulierung. Die Risikovorsorge ist dabei freilich dynamisiert. Belange des Vertrauensschutzes und der Investitionssicherheit werden durch den sozialen Bezug der Betätigung relativiert.

26. Präventive Risikosteuerung ist Zukunfisvorsorge mit „offenem Suchhorizont" und zielt auf die Minimierung des (noch) unspezifischen Risikopotentials umwelt- und gesundheitsrelevanter Aktivitäten. Sie gründet in der Aufiragsdimension des Art. 20 a GG. Geeignete Instrumente sind ein generelles „ökologisches Erforderlichkeitsgebot", ein allgemeines Stoff- und Energiestrommanagement, das Haftungsrecht sowie die Risikokommunikation. Neben der Gewährleistung „ökologischer Markttransparenz" ist dabei eine politische Verständigung auf risikobezogene Leitbilder weiterführend. Diese verkörpern Ideen, aus denen die Inspiration zu gemeinschaftsverträglichen Innovationen erwächst und die Bereitschaft, die Risiken der Risikosteuerung zu akzeptieren.

\section{Ausblick: Rechtliche Ungewissheitsbewältigung und} ihre Rückwirkungen auf das Recht

27. Der Umgang mit dem Risiko ist auch für das Recht selbst riskant. Die Ungewissheit seines Gegenstandes wirkt in funktionaler, methodischer, theoretischer, dogmatischer und vollzugspraktischer Hinsicht auf das Recht zurück. Es kann bei unsicherer Tatsachenentwicklung nicht Erwartungssicherheit vermitteln, sondern nur den Umgang mit der Ungewissheit strukturieren. Im Recht wird die Risikogesellschaft ,auf der Suche nach der verlorenen Sicherheit" trotz aller Reduktion, Prävention und Reflexion deshalb letzllich nicht fündig. 
Dritter Beratungsgegenstand:

\section{Risikosteuerung durch Verwaltungsrecht: Ermöglichung oder Begrenzung von Innovationen?}

2. Bericht von Prof. Dr. Oliver Lepsius, Bayreuth

Inhalt

I. Risiko als Zurechnungsproblem: Schadenswahrscheinlichkeit oder Schadensmöglichkeit? . . . . . . . . . . . . 266

1. Risikobegriffe in der Rechtswissenschaft . . . . . . . 267

a) Dreistufenmodell: Abgrenzung nach Wahrscheinlichkeit . . . . . . . . . . . . . . 268

b) Zweistufenmodell: Abgrenzung nach Möglichkeit . . . 269

2. Risikobegriffe in der Gesetzgebung . . . . . . . . . . 271

a) Der Wandel vom wahrscheinlichen zum möglichen Schaden im deutschen Recht . . . . . . . . . . 272

b) Die Rückkehr zum wahrscheinlichen Schaden im Europarecht . . . . . . . . . . . . . 273

c) Auswirkung auf das Verständnis des Vorsorgeprinzips . 275

II. Risiko als ungewisse Möglichkeit . . . . . . . . . . . 277

1. Verhalten statt Technik als Regelungsgegenstand . . . 278

2. Subjektive statt objektiver Erkennbarkeit technischer Ungewissheit . . . . . . . . . . . . . 279

3. Experimente und flexibles Erfahrungswissen . . . . . 282

III. Die Entindividualisierung der Zurechnung . . . . . . . . . . 283

1. Risiko als Naturalisierung und Vergesellschaftung der Zurechnung . . . . . . . . . . . . . 283

2. Rezeption sozialwissenschaftlicher Problemwahrnehmungen $\ldots \ldots \ldots \ldots \ldots \ldots \ldots \ldots$

3. Rückkehr zu individualisierenden Zurechnungen als soziale Leistung des Rechts . . . . . . . . . . . . . . 289

IV. Risiko als ungewisse Wahrscheinlichkeit . . . . . . . . . . 290

1. Relations- und Selektionsbedürftigkeit . . . . . . . . . 291

2. Normative Nachverdichtung der offenen Relationierung des Gefahrenbegriffs . . . . . . . . . . . . . . . 293 
3. Instrumentelle Umsetzung auf der Ebene des einfachen Rechts . . . . . . . . . . . . . . . . 295

a) Adressatentypisierung, Handlungstypisierung, Erfolgstypisierung . . . . . . . . . . . . . . 295

b) Instrumentenvielfalt . . . . . . . . . . . . . . . 295

c) Unzweckmäßige Relationierungen . . . . . . . . 298

4. Institutionelle Umsetzung auf der Ebene des Verfassungsrechts ................ 300

a) Umsetzung als juristische Rationalität individuellkonkreter Rechtsetzung . . . . . . . . . . . . . 301

b) Umsetzung als politische Rationalität abstraktgenereller Rechtsetzung . . . . . . . . . . . . . . . . 303

V. Der Mensch als Risiko? . . . . . . . . . . . . . . . . 307 


\section{Risiko als Zurechnungsproblem: Schadenswahrscheinlichkeit oder Schadensmöglichkeit?}

Risiko stellt das Recht vor ein prinzipielles Problem: Gefahren werden nicht mehr individuell zugerechnet, sondern kollektiviert. Sie sind nicht mehr Ergebnisse von Handlungen, sondern von sozialen, technischen oder kognitiven Prozessen. Solange Risiken individuell zu verantworten sind, stellen sie kein besonderes, sondern bloß ein Alltagsproblem der Rechtsordnung dar.' Das Risiko im Straßenverkehr ist groß. Gleichwohl verursacht es kein prinzipielles Rechtsproblem, solange es individuell zurechenbar bleibt. Der Mensch lernt mit Risiken umzugehen. Dabei hilft ihm die faktische Erfahrungssicherheit des Lebens und die normative Erwartungssicherheit des Rechts. Risiken sind intendierte Folgen subjektiver Verhaltensentscheidungen. ${ }^{2}$ Hätten menschliche Entscheidungen keine ungewissen Folgen, wäre der Mensch in seinen Entscheidungen nicht frei. Risiken sind die Kehrseite der Freiheit.

Die Ungewissheit einer Entscheidungssituation ${ }^{3}$ ist daher keine Herausforderung moderner Technologien des ausgehenden 20. Jahrhunderts, ${ }^{4}$

\footnotetext{
${ }^{1}$ Dass individuelle Risiken als prinzipiell beherrschbar und erkennbar angesehen werden, zeigt im Umkehrschluss die Abgrenzung der hinreichenden Wahrscheinlichkeit beim polizeirechtlichen Gefahrenbegriff. „Hinreichend wahrscheinlich“ kann der Schadenseintritt erst sein, wenn er das allgemeine Lebensrisiko überschreitet. Dann ist das Risiko nicht mehr sozialadäquat, so dass von einer polizeirechtlichen Gefahr gesprochen wird; vgl. C. Gusy Polizeirecht, 5. Aufl. 2003, Rn. 109. Vgl. auch die Schilderung kollektiver Gefahren als Risiken durch W. Hassemer Polizei im Rechtsstaat, FS Mangakis, 1999, 621, 628.

${ }^{2}$ Vgl. R. Castel Von der Gefährlichkeit zum Risiko, in: M. M. Wambach (Hrsg.) Der Mensch als Risiko, 1983, 51, 59, 61; E. Riehle Sicherheit im Vorfeld des Rechts, ebd., 274, 275, 279. Ähnlich die Differenzierung von K. P. Japp Soziologische Risikotheorie, 1996, 61, 68: Risiko besteht in der Selbstzurechnung von Schäden in Differenz zur Gefahr als Fremdzurechnung von Schäden; sowie N. Luhmann Soziologie des Risikos, 1991, 30f., 117: Risiken sind Schäden, die durch Entscheidungen verursacht werden, Gefahren sind extern veranlasste Schäden der Umwelt. Dieser Unterscheidung schließt sich die juristische Literatur zum Risikoproblem, auch wenn sie systemtheoretisch inspiriert ist, jedoch nicht an, vgl. etwa U. Di Fabio Risikoentscheidungen im Rechtsstaat, 1994, 56f.; K.-H. Ladeur Das Umweltrecht der Wissensgesellschaft, 1995, 72ff. Zur Differenz sozialwissenschaftlicher und juristischer Risikoverständnisse C. Prittwitz Strafrecht und Risiko, 1993, $49 \mathrm{ff} ., 115 \mathrm{ff}$.

${ }^{3}$ Dazu schon W. Berg Die verwaltungsgerichtliche Entscheidung bei ungewissem Sachverhalt, 1980, 245 ff.; I. Spiecker gen. Döhmann Staatliche Entscheidungen unter Unsicherheit, in: J. Lege (Hrsg.) Gentechnik im nicht-menschlichen Bereich - was kann und was sollte das Recht regeln?, 2001, 51, 53f.

${ }^{4}$ Zur Regelung technischer Innovationen in rechtshistorischer Perspektive $M$. Vec Kurze Geschichte des Technikrechts, in: M. Schulte (Hrsg.) Handbuch des Technikrechts, 2003, 3; ders. Technik oder Recht? Steuerungsansprüche in der Zweiten Industriellen Revolution, in: M. Kloepfer (Hrsg.) Kommunikation - Technik - Recht, 2002,
} 
sondern ein altes Erkenntnisproblem. ${ }^{5} \mathrm{Zu}$ einem Rechtsproblem werden Risiken erst in dem Moment, in dem die Zurechnung entindividualisiert wird. ${ }^{6}$ Dann genügen die juristischen Verantwortlichkeiten nicht mehr: Die Umweltverschmutzung lässt sich in weiten Teilen nicht mehr individualisierbaren Handlungen zuordnen, sondern ist ein gesellschaftliches Phänomen. Die Technik als solche kann gefährlich sein, auch wenn der Anlagenbetreiber noch so umsichtig handelt. Wirkungszusammenhänge sprengen individuelle Handlungsbeiträge. Sie sind naturwissenschaftlich komplex und haben grenzüberschreitende und generationenübergreifende Dimensionen. Die Leistung des Risikobegriffs ist es, höchst unterschiedliche Fragestellungen als Problem komplexer Kausalität zu verallgemeinern und als disziplinenübergreifendes Erkenntnisproblem zu formulieren. ${ }^{7}$

\section{Risikobegriffe in der Rechtswissenschaft}

In der Rechtswissenschaft wurde das Zurechnungsproblem in erster Linie in Abgrenzung zum Gefahrenbegriff thematisiert. Die Gefahr setzt die hinreichende Wahrscheinlichkeit einer Rechtsgutverletzung voraus, die einem Störer zugerechnet werden kann. Beim Risiko sind alle diese Merkmale ungewiss. Die Rechtsgutverletzung ist ungewiss. Sie kann

111 ; I. vom Feld Vertrauen ist gut, Kontrolle ist besser? Dampfkesselüberwachung in Preußen zwischen Fremdsteuerung und Selbststeuerung 1870-1914, in: B. Feldner u.a. (Hrsg.) Ad Fontes, 2002, 121; zur Dampfkesselüberwachung auch $R$. WolfDer Stand der Technik, 1986, 57-79. Algemein M. Kloepfer (Hrsg.) Technikentwicklung und Technikrechtsentwicklung, 2000; $R$. Gröschner Das Überwachungsrechtsverhältnis, 1992, 22 ff., $46 \mathrm{ff}$; allgemeiner $M$. Stolleis Die Entstehung des Interventionsstaates und das öffentliche Recht, ZNR 11 (1989), 129; zur Selbstnormierung $M$. Vec Aushöhlung des Staates? Selbst-Normierung im Staat der Industriegesellschaft als historisches Problem, RJ 19 (2000), 517; zum Vollzugsmodell $M$. Stolleis Was bedeutet „Normdurchsetzung “ bei Policeyordnungen der frühen Neuzeit?, FS Landau, 2000, 739. Ein schier unerschöpfliches Quellenreservoir sind die Bände des Archivs für Eisenbahnwesen (1878ff.): ein frühes Zeugnis interdisziplinärer und rechtsvergleichender Technikforschung.

${ }^{5} \mathrm{Vgl}$. J. Franklin The Science of Conjecture. Evidence and Probability before Pascal, 2001, insbes. Kap. 2, 4. Der Mathematiker Franklin weist nach, dass die moderne Wahrscheinlichkeitsrechnung tiefe Wurzeln in der mittelalterlichen römisch-kanonischen Rechtswissenschaft und der spanischen Spätscholastik hat.

${ }^{6}$ Ähnlich U. K. Preuß Risikovorsorge als Staatsaufgabe, in: D. Grimm (Hrsg.) Staatsaufgaben, 1994, 523, 527; K.-H. Ladeur Risikowissen und Risikoentscheidung, KritV 1991, 241, 242; F-X. Kaufmann Sicherheit als soziologisches und sozialpolitisches Problem, 2. Aufl. 1973, 341 ff. Vgl. auch unten III. 1. (mit Nachweisen Fn. 61).

7 Vgl. G. Bechmann (Hrsg.) Risiko und Gesellschaft. Grundlagen und Ergebnisse interdisziplinärer Risikoforschung, 2. Aufl. 1997; G. Banse/G. Bechmann Interdisziplinäre Risikoforschung, 1998, mit umfangreichem bibliographischen Anhang. 
morgen, in hundert Jahren oder gar nicht eintreten. Weder ist absehbar, welche und wessen Rechtsgüter gefährdet sind, noch welche Wirkungszusammenhänge Ursachenketten auslösen. Daher können auch individualisierbare Störer bei Risiken nicht ohne weiteres ausgemacht werden. Die zeitliche Unumkehrbarkeit ${ }^{8}$ und räumliche Beliebigkeit technischer Entwicklungen scheint die Sicherheitstektur des Ordnungsrechts durcheinander zu bringen. Es ist jedoch nicht klar, auf welchen komplexen Ausgangstatbestand der Risikobegriff bezogen werden soll. Hier konkurrieren zwei Grundverständnisse, die entweder auf die Schadenswahrscheinlichkeit oder die Schadensmöglichkeit abstellen.

\section{a) Dreistufenmodell: Abgrenzung nach Wahrscheinlichkeit}

Das erste Modell versteht Risiko als eine Gefahr mit geringerer Wahrscheinlichkeit und ist von dem Bestreben geprägt, Risiko als Rechtsproblem in die traditionelle Systematik einzuordnen. Es ist in der Auseinandersetzung mit der Kernenergie um 1980 entstanden. ${ }^{9}$ Abgrenzungskriterium ist dabei der Grad der Wahrscheinlichkeit. ${ }^{10}$ Während für die Gefahr eine hinreichende Wahrscheinlichkeit erforderlich ist, betreffen Risiken eine graduell niedrigere Wahrscheinlichkeit. Im Ergebnis entsteht ein Dreistufenmodell, das Gefahr, Risiko und Restrisiko nach Wahrscheinlichkeitsgraden unterscheidet. ${ }^{11}$ Dies gelingt im Hinblick auf das Restrisiko:

${ }^{8}$ P. Hiller Der Zeitkonflikt in der Risikogesellschaft. Risiko und Zeitorientierung in rechtsförmigen Verwaltungsentscheidungen, 1993, 45ff.: „Zukunftsorientierung des Risikos - Vergangenheitsorientierung des Rechts“. Diese Perspektive konzentriert sich einseitig auf die Rechtsanwendung und vernachlässigt die Rechtsetzung. Demgegenüber die Gesetzgebung als klassisches juristisches Instrumentarium zeitübergreifender Regelungen begreifend H. Schulze-Fielitz Zeitoffene Gesetzgebung, in: W. Hoffmann-Riem/ E. Schmidt-Aßmann (Hrsg.) Innovation und Flexibilität des Verwaltungshandelns, 1994, $139,151 \mathrm{ff} ., 183 \mathrm{ff}$.

9 BVerfGE 49, 89, 137-143; 53, 30, 59; R. Breuer Gefahrenabwehr und Risikovorsorge im Atomrecht, DVB1 1978, 829, 835 f.; ders. Anlagensicherheit und Störfalle - Vergleichende Risikobewertung im Atom- und Immissionsschutzrecht, NVwZ 1990, 211, 213; B. Bender Gefahrenabwehr und Risikovorsorge als Gegenstand nukleartechnischen Sicherheitsrechts, NJW 1979, 1425; R. Steinberg Atomrechtliche Schadensvorsorge und „Restrisiko“, in: H.-P. Schneider/ders. Schadensvorsorge im Atomrecht, 1991, 9, $13 \mathrm{ff}$.

${ }^{10}$ A. Reich Gefahr - Risiko - Restrisiko, 1989, $75 \mathrm{ff}$.

1 Vgl. D. Rauschning Staatsaufgabe Umweltschutz, VVDStRL 38 (1980), 167, 192f.; $R$. Wolf Technik (Fn. 4), 290ff.; F. Ossenbühl Vorsorge als Rechtsprinzip im Gesundheits-, Arbeits- und Umweltschutz, NVwZ 1986, 161, 162f.; $M$. Ronellenfitsch Die Bewältigung der wissenschaftlichen und technischen Entwicklungen durch das Verwaltungsrecht, DVBI 1989, 851, 859; J. Ipsen Die Bewältigung der wissenschaftlichen und technischen Entwicklungen durch das Verwaltungsrecht, VVDStRL 48 (1990), 177, 186f.; R. Wahl/ I. Appel Prävention und Vorsorge, in: R. Wahl (Hrsg.) Prävention und Vorsorge, 1995, 1, $84 \mathrm{ff}$; E. Rehbinder Ziele, Grundsätze, Strategien und Instrumente, in: J. Salzwedel u.a. 
Als bloße, hypothetische Möglichkeit ist es unwahrscheinlich und daher kein rechtlicher Regelungsgegenstand. Schwierigkeiten bereitet es jedoch, den Bereich des Risikos von der Gefahr über eine graduell abgestufte Wahrscheinlichkeit abzugrenzen.12 Die Abgrenzung wird nicht tatbestandlich, sondern mit Blick auf die Rechtsfolgen vorgenommen. Risiken lösen keine Maßnahmen der Gefahrenabwehr, sondern nur vorsorgende Minimierungsmaßnahmen aus. ${ }^{13}$ Letztlich entscheiden die Rechtsfolgen über die Tatbestandskonkretisierung, ohne dass innerhalb des Wahrscheinlichkeitsurteils eine normative Grenze eingezogen werden kann. Risiko bleibt ein normativ undefinierter Bereich unwahrscheinlicher Gefahren.

\section{b) Zweistufenmodell: Abgrenzung nach Möglichkeit}

Diesem Mangel hilft der zweite rechtswissenschaftliche Risikobegriff ab. Er dreht das Verhältnis der Kategorien um und erklärt Risiko zum Oberbegriff. Hieraus folgt ein Zweistufenmodell, das ein neues Abgren-

(Hrsg.) Grundzüge des Umweltrechts, 2. Aufl. Stand 1997, Teil 04 Rn. 20-26; R. Steinberg Der ökologische Verfassungsstaat, 1998, 102-104; W. Köck Rationale Risikosteuerung als Aufgabe des Rechts, in: E. Gawel (Hrsg.) Effizienz im Umweltrecht, 2001, 271, 280-283.

12 Das BVerwG trifft eine Abgrenzung, die das Verhältnis von Wahrscheinlichkeit und Möglichkeit jedoch nicht klärt, sondern die Regelungserwartung von der Gefahrenabwehr auf die Risikovorsorge ausdehnt. Die Rechtsprechung des 7. Senats prägt die Wahrnehmung bis heute. Vgl. BVerwGE 69, 37, 43: „Vorsorge im Sinne von $\S 5$ Nr. 2 BImSchG ist daher jedenfalls dann geboten, wenn hinreichende Gründe für die Annahme bestehen, daß Immissionen möglicherweise zu schädlichen Umwelteinwirkungen führen und damit - auch wenn sich entsprechende Ursachenzusammenhänge im einzelnen noch nicht eindeutig feststellen lassen - ein Gefahrenverdacht besteht." (Hervorhebung im Original.) Die Aussage ist insofern in sich widersprüchlich, als ein Gefahrenverdacht eine wahrscheinliche Gefahr voraussetzt und dann nicht auf die pure Möglichkeit bezogen sein kann. Wahrscheinlichkeit und Möglichkeit vermengend auch derselbe Senat BVerwGE 72, 300, 315: „Vorsorge bedeutet des weiteren, daß bei der Beurteilung von Schadenswahrscheinlichkeiten nicht allein auf das vorhandene ingenieurmäßige Erfahrungswissen zurückgegriffen werden darf, sondern Schutzmaßnahmen auch anhand ,bloß theoretischer Überlegungen und Berechnungen in Betracht gezogen werden müssen, um Risiken aufgrund noch bestehender Unsicherheiten oder Wissenslücken hinreichend zuverlässig auszuschließen." Wenn Gefahren und Risiken praktisch ausgeschlossen sein müssen, wird die Regelungserwartung so weit ausgedehnt, dass eine entsprechende Reduzierung der Kontrolldichte durch das Gericht unvermeidlich ist. Die Entwicklung des „exekutiven Beurteilungsspielraums“ durch das Gericht ist die institutionelle Konsequenz einer ausgedehnten Regelungserwartung auf mögliche Schäden, für die rechtliche Prüfungskriterien entfallen.

13 Statt vieler $R$. Wahl Risikobewertung und Risikobewältigung im Lebensmittelrecht, ZLR 1998, 275, 282. 
zungskriterium einführt: die Möglichkeit des Schadenseintritts. Danach bleibt die Gefahr auf die Wahrscheinlichkeit begrenzt, während das Risiko auf die Möglichkeit einer Beeinträchtigung bezogen wird. ${ }^{14}$ Nun sind zwar Gefahr und Risiko ihrem Anspruch nach geschieden, dafür entsteht ein neues Abgrenzungsproblem zum Restrisiko. Ein prinzipiell hinzunehmendes Restrisiko kann es nicht mehr geben, weil die Grenze des Hinnehmbaren selbst ungewiss ist. Erst durch das Möglichkeitskriterium kann sich der Risikobegriff vom Gefahrenbegriff emanzipieren und eine eigenständige Qualität gewinnen.

Dieser Risikobegriff ist in den 1990er Jahren entstanden ${ }^{15}$ und hat sich in der deutschen Literatur inzwischen durchgesetzt. ${ }^{16}$ Der Rechtswissen-

${ }_{14} \mathrm{Ma}$ sstab setzend die Definitionen in $M$. Kloepfer/E. Rehbinder/E. Schmidt-Aß-
mann/P. Kunig Umweltgesetzbuch - Allgemeiner Teil, 1990, $\$ 2$ Abs. 6: „Umweltrisiko
im Sinne dieses Gesetzes ist die Möglichkeit des Eintritts einer Umweltbeeinträchtigung,
soweit sie nicht aufgrund praktischer Vernunft ausgeschlossen erscheint. Umweltgefahr
ist dasjenige Umweltrisiko, welches unter Berücksichtigung des Grades seiner Eintritts-
wahrscheinlichkeit und des möglichen Schadensumfanges nicht mehr hinnehmbar ist.“
Dazu die Erläuterungen ebd., 119f. in Anlehnung an D. Murswiek Die staatliche Verant-
wortung für die Risiken der Technik, 1985, 85 , nach dem jede Möglichkeit des Schadens-
eintritts ein Risiko darstellt. Modifizierte Definitionen enthält der Entwurf der Unabhän-
gigen Sachverständigenkommission, vgl. Bundesministerium für Umwelt, Naturschutz
und Reaktorsicherheit (Hrsg.) Umweltgesetzbuch (UGB-KomE), 1998, § 2 Nr. 4 und 5
UGB-KomE: ,4. Gefahr: die hinreichende Wahrscheinlichkeit des Eintritts einer erheb-
lichen nachteiligen Einwirkung auf ein Schutzgut dieses Gesetzbuches; je gewichtiger die
zu besorgende Einwirkung ist, desto geringer sind die an die Eintrittswahrscheinlichkeit
zu stellenden Anforderungen; 5 . Risiko: die Möglichkeit des Eintritts einer nicht nur ge-
ringfügigen nachteiligen Einwirkung auf ein Schutzgut dieses Gesetzbuches, soweit sie nicht praktisch ausgeschlossen erscheint“. Dazu Erläuterungen 440-442.

${ }^{15}$ Zur Entwicklungsgeschichte und Neuorientierung um $1990 \mathrm{R}$. Wahl in: R. Landmann/G. Rohmer/K. Hansmann (Hrsg.) Umweltrecht, Band IV, 20. Lfg. 1994, § 6 GenTG Rn. 13-31; K.-H. Ladeur Risiko und Recht. Von der Rezeption der Erfahrung zum Prozeß der Modellierung, in: Risiko und Gesellschaft (Fn. 7), 209, 209-214; H.-H. Trute Staatliches Risikomanagement im Anlagenrecht, in: E. Riedel (Hrsg.) Risikomanagement im öffentlichen Recht, 1997, 55, 95, 98, 100.

${ }^{16}$ D. Murswick Die Bewältigung der wissenschaftlichen und technischen Entwicklungen durch das Verwaltungsrecht, VVDStRL 48 (1990), 207, 212; $R$. Wahl/I. Appel Prävention (Fn. 11), 88; H.-H. Trute Risikomanagement (Fn. 15), 100; F. OssenbühlDie Not des Gesetzgebers im naturwissenschaftlich-technischen Zeitalter, 2000, 27; C. Calliess Vorsorgeprinzip und Beweislast im Verwaltungsrecht, DVBI 2001, 1725, 1727; A. Karthaus Risikomanagement durch ordnungsrechtliche Steuerung, 2001, 58; U. Prall Gentechnikrecht, in: H.-J. Koch (Hrsg.) Umweltrecht, 2002, § 11 Rz. 93; U. Di Fabio Risikovorsorge - uferlos?, ZLR 2003, 163, 164, 166; W. Kahl Risikosteuerung durch Verwaltungsrecht, DVBI 2003, 1105, 1108; H. Schulze-Fielitz Technik und Umweltrecht, in: M. Schulte (Hrsg.) Handbuch des Technikrechts, 2003, 443, 461. 
schaft ist es damit gelungen, einen Rechtsbegriff des Risikos zu etablieren, allerdings um den Preis, dass dieser auf die Möglichkeit und nicht die Wahrscheinlichkeit des Schadens bezogen wird. ${ }^{17}$

\section{Risikobegriffe in der Gesetzgebung}

In der Gesetzgebung wurde der Begriff des Risikos anfänglich zurückhaltend verwendet ${ }^{18}$ und hat durch europarechtlich induzierte Gesetzesänderungen erst in jüngerer Zeit verstärkt Eingang in die Gesetzessprache gefunden. ${ }^{19}$ Der Wandel vom Wahrscheinlichkeitsbezug zum Möglichkeitsdenken lässt sich auch in der Gesetzgebung nachvollziehen. ${ }^{20}$

17 Vgl. K.-H. Ladeur Das Umweltrecht der Wissensgesellschaft, 1995, 72f., 76ff.: Die grundsätzlich zu begrüßende Verselbständigung des Risikobegriffs wird mit einer Höherstufung der Abstraktionsebene erkauft, die einen genaueren Zugriff auf die Bedeutung der Ungewissheit für eine schärfere Fassung des Risikobegriffs verfehlt (77).

18 Noch 1985 konnte D. Murswick Verantwortung (Fn. 14), 80, schreiben, Risiko sei kein Rechtsbegriff, denn die Gesetzessprache verwende ihn nicht, und auch noch 1989 konnte R. Breuer Anlagensicherheit (Fn. 9), 213, feststellen, der Begriff des Risikos tauche in keinem Gesetz auf.

19 Z.B. $\$ 28$ Abs. 3 c S. $1, \S 40$ Abs. 1 S. 1 Nr. $1, \S 62$ S. $1, \S 63$ a Abs. 1 AMG; $\$ 7$ Abs. $2 a$ AtG a.F.; $\S 3$ b Abs. 1 Nr. $4, \S 12$ a S. 2 Nr. $1, \S 12$ b Abs. 4 S. 1 und S. 2 , $\S 12$ f Abs. 1 S. 1 , $\S 12$ h Abs. 2 S. $3, \S 15$ a Abs. 2 S. $3, \S 17$ Abs. 2 , $\$ 19$ Abs. 3 Nr. 2 ChemG; $\S 2$ Abs. 1 Nr. 4 , $\S 6$ Abs. 1 und Abs. $2, \S 7$ Abs. 1 S. 1 und S. $3, \S 10$ Abs. 2 S. 2 Nr. $5, \S 21$ Abs. 5 GenTG; $\S 15$ a Abs. $2, \S 16$ Abs. 2, $\S 36$ Abs. 2 S. 1 GefStoffV; Nr. 1.5 Anlage 2 UVPG.

20 Die wesentlichen gesetzlichen Stufen in der Entwicklung der unterschiedlichen Vorsorge- und Risikokonzeptionen werden durch folgende Tatbestände verkörpert: $\$ 7$ Abs. 2 Nr. 3 AtomG spricht von einer ,Vorsorge gegen Schäden“ und impliziert damit die Wahrscheinlichkeit des Schadenseintritts. Die Vorschrift bleibt dem System der Gefahrenabwehr verhaftet. $\S 5$ Abs. 1 Nr. 2 BImSchG lockert diesen Zusammenhang einerseits durch die Formulierung „Vorsorge gegen schädliche Umwelteinwirkungen“, hält ihn andererseits durch die hinzugefügten Worte „und sonstige Gefahren“ aufrecht. Ausdehnend ist auch $\S 5$ Abs. 1 Nr. 1 BImSchG, der einen Wirkungsstandard verlangt, nach dem Schäden nicht hervorgerufen werden können. Hier wird eine illusionäre Möglichkeitssteuerung bezweckt. Das Wort „können“ wurde auf Vorschlag des Bundesrates eingefügt, vgl. A. Roßnagel GK-BImSchG, § 5 Rn. 155; F. Petersen Schutz und Vorsorge, 1993, $116 \mathrm{ff}$., 155. $\mathrm{Zu}$,potentiell schädlichen“ Umwelteinwirkungen als rechtlichem Regelungsgegenstand H. D. Jarass BImSchG, 5. Aufl. 2002, § 3 Rn. 23. Die weitere Abkehr von der wahrscheinlichkeitsorientierten Gefahrenabwehr zur möglichkeitsorientierten Risikovorsorge bringt $\S 1 \mathrm{Nr}$. 1 GenTG (Schutz vor „möglichen Gefahren“), sowie $\S 6$ Abs. 1 GenTG. Nicht zuletzt die „Sicherheitsstufe 1“ nach $\$ 7$ Abs. 1 Nr. 1 GenTG drückt die Ausdehnung der Vorsorge aus. Obwohl hier kein Risiko besteht, ordnet das Gesetz den ungefährlichen Zustand gleichwohl einer "Sicherheitsstufe“ zu. Nach R. Scholz Verfassungsfragen der Gentechnik, FS Sendler, 1991, 93, 101, wird hier „das Prinzip der sicherheitsrechtlichen Prävention in einem Übermaß strapaziert, das verfassungsrechtlich kaum zu rechtfertigen ist". 


\section{a) Der Wandel vom wahrscheinlichen zum möglichen Schaden im deutschen Recht}

Die deutsche Rechtsentwicklung ist durch die Ausdehnung der Gefahrenabwehr von bloß geringeren Schadenswahrscheinlichkeiten im Atom-21 und Arzneimittelgesetz $^{22}$ auf die Abwehr möglicher Schäden gekennzeichnet. Dieser Konzeptwandel manifestiert sich im 1990 verkündeten Gentechnikgesetz, ${ }^{23}$ das dem Schutz vor der Möglichkeit eines Schadens den Vorrang einräumt. ${ }^{24}$ Jetzt geht der Schutzzweck dem Förderzweck vor. Das Gentechnikgesetz ist das legislatorische Dokument des Wandels zum Zweistufenmodell. ${ }^{25}$

${ }^{21}$ Dazu H.-W. Rengeling Reaktorsicherheit, Vorsorge auch jenseits praktischer Vernunft?, DVBI 1988, 257; R. Steinberg Atomrechtliche Schadensvorsorge (Fn. 9), $9 \mathrm{ff}$; U. Di Fabio Risikoentscheidungen (Fn. 2), 73 ff.; $R$. Schröder Verfassungsrechtliche Rahmenbedingungen des Technikrechts, in: Handbuch des Technikrechts (Fn. 16), 185, 196-200.

22 Dazu U. Di Fabio Risikoentscheidungen (Fn. 2), $166 \mathrm{ff}$.

${ }^{23}$ Zur Entstehung und Zielsetzung des GenTG J. Lege Das Recht der Bio- und Gentechnik, in: Handbuch des Technikrechts (Fn. 16), 669, 680ff., 693ff.; ders. Die Steuerung der Gentechnik durch Recht, in: M. Kloepfer (Hrsg.) Technikumsteuerung als Rechtsproblem, 2002, 67, 74 ff.; $R$. Wahl (Fn. 15), Vorbem. GenTG Rn. 21-38; R. Scholz Verfassungsfragen (Fn. 20), 96ff.; $R$. Lukes Der Entwurf eines Gesetzes zur Regelung von Fragen der Gentechnik, DVBl 1990, 273; Enquete-Kommission des Deutschen Bundestages „Chancen und Risiken der Gentechnologie“ BT-Drs. 10/6775, dazu W.-M. Catenhusen/H. Neumeister (Hrsg.) Chancen und Risiken der Gentechnologie. Dokumentation des Berichts an den Deutschen Bundestag, 1990. Vgl. auch die den Gesetzesvorbehalt aus Schutzpflichten einfordernde Entscheidung des VGH Kassel, NJW 1990, 337 = DVB1 1990, 63, dazu M. Rose Gentechnik und Vorbehalt des Gesetzes, DVBI 1990, 279.

${ }^{24}$ Vgl. den Zielkonflikt zwischen § 1 Nr. 1 GenTG (Schutz vor möglichen Gefahren der Gentechnik; Vorbeugung gegenüber dem Entstehen solcher Gefahren) und $\S 1$ Nr. 2 GenTG (Förderung der Gentechnik); dazu W. Graf Vitzthum/T. Geddert-Steinacher Der Zweck im Gentechnikrecht, 1990, 38ff.; $R$. Wahl (Fn. 15), 26. Lfg. 1997, §1 GenTG Rn. 7, 10-12. Nach hM geht der Schutzzweck dem Förderzweck vor, vgl. $R$. Huth Gentechnik und Umweltrechtskodifikation, 2001, 286ff.; R. Wahl/H. Melchinger Das Gentechnikrecht nach der Novellierung, JZ 1994, 973, 979f.; U. Di Fabio Bio- und Gentechnikrecht, in: H.-W. Rengeling (Hrsg.), Handbuch zum europäischen und deutschen Umweltrecht, Band II, 1998, § 64 Rn. 67.

${ }^{25}$ R. Wahl(Fn. 15), Rn. 19, spricht von einer Neuorientierung der Sicherheitsdogmatik durch das GenTG. Vgl. auch A. Meier Risikosteuerung im Lebensmittel- und Gentechnikrecht, 2000, 24ff.; I. Appel Stufen der Risikoabwehr, NuR 1996, 227; W. Graf Vitzthum Zur Gentechniknovelle 1993, ZG 1993, 236; K.-H. Ladeur Gefahrenabwehr und Risikovorsorge bei der Freisetzung von gentechnisch veränderten Organismen nach dem Gentechnikgesetz, NuR 1992, 254, 257 ff.; G. Winter Entfesselungskunst, KJ 1991, 18. 


\section{b) Die Rückkehr zum wahrscheinlichen Schaden im Europarecht}

Eine andere Entwicklung lässt sich dem Europarecht entnehmen, dessen Risikobegriffe ${ }^{26}$ zwar die Ausdehnung auf die Möglichkeit ursprünglich zuließen, jetzt aber durch eine Rückkehr zum Wahrscheinlichkeitsbezug gekennzeichnet sind. Die in Deutschland erreichte terminologische Differenzierung wird dadurch in Frage gestellt, dass etwa in der Novel Food-Verordnung ${ }^{27}$ oder der Lebensmittel-Basisverordnung 28 nicht auf die Möglichkeit, sondern auf die Wahrscheinlichkeit abgestellt wird. ${ }^{29}$ Terminologisch unklarer ${ }^{30}$ sind die Richtlinien im Gentechnik-

\footnotetext{
${ }^{26}$ Überblicksdarstellungen bei $M$. Schröder Umweltschutz als Gemeinschaftsziel und Grundsätze des Umweltschutzes, in: H.-W. Rengeling (Hrsg.) Handbuch zum europäischen und deutschen Umweltrecht, Band 1, 2. Aufl. 2003, §9 Rn. 34 ff. mwN.; U. Di Fabio Bio- und Gentechnikrecht (Fn. 24), § 64 Rn. $4 \mathrm{ff}$., $82 \mathrm{ff}$., $165 \mathrm{ff}$.; allgemein L. Krämer EC-Treaty and Environmental Law, 3. Aufl. 1998; A. Epiney Umweltrecht in der Europäischen Union, 1997.

27 Art. 3 Abs. 1 der Novel-Food-Verordnung, 258/97/EG v. 27. 1. 1997, verwendet den Begriff „Gefahr“. Zur Kritik an der Novel Food-Verordnung, nicht zuletzt ihrer Begrifflichkeit: R. Streinz (Hrsg.) „Novel Food“: rechtliche und wirtschaftliche Aspekte der Anwendung neuer biotechnologischer Verfahren bei der Lebensmittelherstellung, 2. Aufl. 1995; ders. (Hrsg.) Neuartige Lebensmittel, 1999; U. Di Fabio Bio- und Gentechnikrecht (Fn. 24), § $64 \mathrm{Rn}$. 165ff., 186ff.; R. Wahl/D. Groß Die Europäisierung des Genehmigungsrechts am Beispiel der Novel Food-Verordnung, DVBl 1998, 2, 9; W. Berg Riskmanagement im Rechtsstaat am Beispiel der Novel Food-Verordnung, ZLR 1998, 375, $377 \mathrm{ff}$; ders. Die Problematik der Verwendung unbestimmter Rechtsbegriffe, dargestellt am Beispiel der „Novel Food“-Verordnung, ZLR 1993, 455. Zu sprachlichen und terminologischen Differenzen beim Risiko- und Gefahrenbegriff $R$. Wahl Risikobewertung (Fn. 13), 278-284, 294-296.

28 Die Lebensmittel-Basisverordnung definiert Risiko über das Merkmal der Wahrscheinlichkeit des Schadenseintritts, vgl. Art. 3 Nr. 9 vO 178/2002/EG v. 28. 1. 2002: Risiko bezeichne ,eine Funktion der Wahrscheinlichkeit einer die Gesundheit beeinträchtigenden Wirkung und der Schwere dieser Wirkung als Folge der Realisierung einer Gefahr.“ In Art. 7 Abs. 1 der Verordnung ist zwar terminologisch von der „Möglichkeit gesundheitsschädlicher Auswirkungen" die Rede, der Sache nach handelt es sich aber um eine in ihrer Höhe noch nicht quantifizierbare Wahrscheinlichkeit. Der Risikobegriff nähert sich hier dem Gefahrenverdacht an. Der Begriff der Gefahr wird demgegenüber auf die Verursachung verengt, vgl. Art. 3 Nr. 14. Ähnlich Art. 3 Nr. 7 RL 1996/82/EG zur Beherrschung der Gefahren bei schweren Unfällen mit gefährlichen Stoffen v. 9. 12. 1996.

29 Daher können $R$. Schweizer/T. Calame Das Gentechnikrecht der Europäischen Gemeinschaft, RIW 1997, 34, 40f. größere Übereinstimmung des europäischen Risikobegriffs mit dem älteren Dreistufen- als dem neueren Zweistufenmodell konstatieren.

${ }^{30}$ Zur unklaren Abgrenzung von Risiko und Gefahr im Gentechnikrecht J. Lege Das Recht der Bio- und Gentechnik, in: Handbuch des Technikrechts (Fn. 16), 693-696; auch schon: R.-D. Drescher Jurassic Park - Made in Germany?, ZUR 1994, 289, 294; G. Hirsch/A. Schmidt-Didczuhn Gentechnikgesetz, 1991, §1 Rn. 12.
} 
recht, ${ }^{31}$ aber auch hier zeichnet sich ein Interpretationswandel zur Wahrscheinlichkeit an Stelle der Möglichkeit ab.32 Insgesamt ist der Risikobegriff im Europarecht trotz inhaltlicher Bandbreite, ${ }^{33}$ uneinheitlicher Übersetzungen ${ }^{34}$ und auch gewisser Widersprüche ${ }^{35}$ durch die Rückkehr zum Grad der Wahrscheinlichkeit geprägt. ${ }^{36}$ Dies tritt noch nicht in vol-

${ }^{31}$ Die Erwägungsgründe der Systemrichtlinie (RL 90/219/EWG v. 23. 4. 1990) sprechen davon, dass die Risiken ,noch nicht vollständig bekannt“ sind, setzen also eine Mindestwahrscheinlichkeit voraus. Art. 5 verwendet den Begriff der "Gefahrenbewertung“, während es in der englischen Fassung, ,risk assessment “ heißt. Nicht eindeutig ist auch die Freisetzungsrichtlinie (neue Fassung) (RL 2001/18/EG v. 12.3. 2001), Erwägungsgründe 5, 18, Art. 2 Nr. 8. Hier ist unklar, ob die Möglichkeit oder die Wahrscheinlichkeit gemeint ist, es wird sowohl von „potentiellen" als auch von „fallweisen“ Risiken gesprochen.

32 Die Systemrichtlinie (90/219/EWG, zuletzt geändert durch RL 98/81/EG) verfolgte ursprünglich einen verfahrensbezogenen Ansatz. Schon die Gefahren der Arbeitsverfahren und nicht erst die Ergebnisse sollten erfasst werden. Mit Art. 3 Abs. 2 der Systemrichtlinie wird nun eine Verschiebung zu einem produktbezogenen Ansatz vorgenommen. Dem liegt die Erkenntnis zugrunde, dass weniger das gentechnische Verfahren, sondern das daraus hervorgegangene Produkt das Risiko enthält. Darin liegt zugleich der Übergang von einer offenen, unkonkretisierten Risikoanalyse zu einem relationierenden Verständnis. Vgl. zu diesen Entwicklungen $M$. Herdegen/H.-G. Dederer Internationale Praxis Gentechnikrecht, 21. Lfg. 2003, Teil 3/I, 1, Rn. 25f. („Lockerung des verfahrensbezogenen Ansatzes“); U. Di Fabio Bio- und Gentechnikrecht (Fn. 24), § 64 Rn. 26.

33 Eine einheitliche Definition ist schon angesichts der Normenvielfalt nicht zu erwarten. Die Begriffe Risiko, Risikoanalyse, Risikobewertung werden in einer Vielzahl von EG-Rechtsakten erwähnt, z.B. Erwägungsgrund 34, Art. 3 Nr. 9, Art. 6 ff., Art. 34, Art. 50 Lebensmittel-Basisverordnung (178/2002/EG); Art. 9 Abs. 1 Novel-Food-Verordnung (258/97/EG); Erwägungsgründe 5, 18, Art. 2 Nr. 8, Anhang II der Freisetzungsrichtlinie (2001/18/EG); Art. 8 sowie Anhang II und V VO zur Verhütung, Kontrolle und Tilgung bestimmter transmissibler spongiformer Enzephalopathien (999/2001/EG mit ÄndVO 1128/2003/EG).

34 Manche Rechtsquellen verwenden auf Deutsch den Begriff Gefahr, auf Englisch oder Französisch den des Risikos, vgl. Anhang VI 6.2 h) der Umweltauditverordnung (761/2001/EG) v. 19. 3. 2001; Erwägungsgründe der Richtlinie zur Bekämpfung der Luftverunreinigung durch Industrieanlagen (84/360/EWG) v. 28. 6. 1984; Art. 5, 7 Abs. 3 Systemrichtlinie $(90 / 219 /$ EWG $)$.

35 Art. 3 Abs. 1 Novel-Food-VO (258/97/EG) spricht von einer Gefahr. In Verbindung mit dem Vorsorgeprinzip könnte auch ein Risiko gemeint sein. Den umgekehrten Fall enthält Annex I Nr. II 12 der Richtlinie für medizinische Geräte (1993/42/EWG), wo von Risiko die Rede ist, obwohl gesicherte Kenntnisse vorliegen dürften.

${ }^{36}$ Deutlich jetzt vor allem EuGH, Urteil v. 9. 9. 2003 zur Novel Food-Verordnung, C-236/01 (Monsanto Agricultura Italia u.a.), Rn. 106-113: Schutzmaßnahmen können nicht wirksam mit einer rein hypothetischen Betrachtung des Risikos begründet werden, die auf bloße, wissenschaftlich noch nicht verifizierte Vermutungen gestützt wird. Stichhaltige Gründe und spezifische Indizien sind erforderlich. Ähnlich auch schon EuG, Urteil v. 11. 9. 2002, T-13/99 (Pfizer Animal Health), Slg. 2002, П-3305 Rn. 139-148, 321: Der Begriff Risiko stelle die Funktion der Wahrscheinlichkeit nachteiliger Wirkungen für 
lem Umfang zu Tage, weil das Verfahren der Risikoanalyse die Ermittlung der Wahrscheinlichkeitsgrade prozeduralisiert und dadurch scheinbar auf die Möglichkeit erstreckt. ${ }^{37}$

\section{c) Auswirkung auf das Verständnis des Vorsorgeprinzips}

Die Differenzen zwischen deutschem und europäischem Recht wirken sich auf die nähere Bestimmung des Vorsorgeprinzips aus. In Deutschland dient das Vorsorgeprinzip zur Erweiterung des Regelungsgegenstandes auf mögliche Gefahren. ${ }^{38} \mathrm{Im}$ Europarecht hingegen dient es dazu, die

das geschützte Rechtsgut aufgrund der Verwendung eines Produktes oder Verfahrens dar. EuG, Urteil v. 11. 9. 2002, T-70/99 (Alpharma), Slg. 2002, II-3495 Rn. 152-161: Hypothesen ohne Indizien genügen nicht für die Risikoanalyse. Bei der Risikobewertung geht es darum, den Grad der Wahrscheinlichkeit der nachteiligen Wirkungen eines bestimmten Produktes oder Verfahrens für die menschliche Gesundheit und die Schwere dieser Wirkungen zu bewerten.

37 Art. 6 Lebensmittel-Basisverordnung (178/2002/EG) sowie der Risikobegriff des Art. 3 Abs. 9, der dem Gefahrenverdacht in deutscher Terminologie entspricht. Die Definitionen von Risikoanalyse, Risikobewertung und Risikomanagement in Art. 3 Abs. 10-12 knüpfen gleichfalls an Wahrscheinlichkeitsurteile an. Auch das Verfahren zur Identifikation neu auftretender Risiken in Art. 34 setzt einen Gefahrenverdacht voraus. Schließlich bezieht auch die Rechtsprechung die Risikobewertung nur auf die Wahrscheinlichkeit, vgl. EuG, Slg. 2002, II-3305, Rn. 129-148; EuG, Slg. 2202, II-3495, Rn. 147-160. Vgl. auch die Rechtsprechung in Fn. 36. Ähnlich schon Mitteilung der Kommission der Europäischen Gemeinschaft v. 30. 4. 1997 (KOM 97, 183 endg.) über „Gesundheit der Verbraucher und Lebensmittelsicherheit“, 7, $20 \mathrm{f}$.

38 Vgl. BVerwGE 69, 37, 43; 72, 300, 315. BT-Drs. 7/1513, 2 zu $§ 5$ BImSchG: Die Vorsorgepflicht diene nicht dem Schutz vor konkret bzw. belegbar schädlichen Umwelteinwirkungen, sondern beuge dem Entstehen solcher Umwelteinwirkungen generell vor. Aus der umfangreichen Literatur A. Helberg in: H.-J. Koch (Hrsg.) Umweltrecht, 2002, $\S 3$ Rn. 12f.; R. Sparwasser/R. Engel/A. Voßkuhle Umweltrecht, 5. Aufl. 2003, §10 Rn. 153 (,potentiell gefährlich“); M. Kloepfer/T. Brandner Umweltrecht, 2. Aufl. 1998, $\S 4$ Rn. 5 ff., 15-17; H. D. Jarass BImSchG, 5. Aufl. 2002, §5 Rn. 46 (,potentielle Schäden“); W. Sandner in: R. Schmidt/T. Vollmöller (Hrsg.) Kompendium Öffentliches Wirtschaftsrecht, 2. Aufl. 2004, §9 Rn. 19; C. Calliess Vorsorgeprinzip (Fn. 16), 1727: Entscheidend sei die Ersetzung der konkreten, hinreichenden Wahrscheinlichkeit durch die reine Möglichkeit, die abstrakte Besorgnis, eines Schadenseintritts; A. Epiney Umweltrecht (Fn. 26), 100: Vorsorge geht über die Gefahrenabwehr hinaus und zielt auf eine Risikovermeidung ab. Nach M. Herdegen/H.-G. Dederer (Fn. 32), Teil 3/I, 2., IV 2., operiere das Vorsorgeprinzip in der Zone wissenschaftlich nicht ausgeleuchteter Gefahrenpotentiale und greife die Erkenntnis der Wissenschaft über die Grenzen menschlichen wissenschaftlichen Erkenntnisvermögens auf. Die Grenzen des Vorsorgeprinzips betonend und seiner Expansion vorbeugend $U$. Di Fabio Voraussetzungen und Grenzen des umweltrechtlichen Vorsorgeprinzips, FS Ritter, 1997, 807, $820 \mathrm{ff}$.: „Jede Vorverlagerung des hoheitlichen Eingriffspunktes in den Bereich der Risikovorsorge schiebt die Beurteilung von Schadensmöglichkeiten in die Grenzregionen des Wissens, verengt demnach den Bereich, der als Restrisiko hingenommen werden muß, weil menschliches Erkenntnisvermögen struk- 
Eingriffsmittel einem spezifischen Verhältnismäßigkeitsmaßstab zu unterwerfen..$^{39} \mathrm{Im}$ Europarecht präzisiert ein engerer Risikobegriff das Vorsor-

turell begrenzt ist. Gerade diese Bewegung zur Restrisikogrenze läßt die Möglichkeiten normativer Steuerung schwinden und ruft die Gefahr hervor, in unkontrollierbare Überlegungen und Abwägungen abzugleiten" (821). Einengend auch R. Breuer Umweltschutzrecht, in: E. Schmidt-ABmann (Hrsg.) Besonderes Verwaltungsrecht, 12. Aufl. 2003, 5. Kap. Rn. 8f., der nicht die materielle, sondern die normative und instrumentelle Ausprägung des Vorsorgeprinzips hervorhebt. Kritisch schon F. Ossenbühl Vorsorge (Fn. 11), 165f., der sich gegen eine „Vorsorge ins Blaue“ wendet und ein Mindestmaß an Realität aufgrund tatsächlicher Anhaltspunkte fordert. Vorsorge-beschränkende Regeln fordert auch $H$. v. Lersner Nichtanthropozentrische Erweiterung des Umweltrechts?, in: J. Nida-Rümelin/D. v. d. Pfordten (Hrsg.) Ökologische Ethik und Rechtstheorie, 2. Aufl. 2002, 191, 196. Kritik am Vorsorgeprinzip bei $W$. van den Daele Zur Reichweite des Vorsorgeprinzips - politische und rechtliche Perspektiven, in: J. Lege (Hrsg.) Gentechnik (Fn. 3), 101, 110-115; G. Lübbe-Wolff Modernisierung des Umweltordnungsrechts, 1996, 71 f., 83-87; R. Fleury Das Vorsorgeprinzip im Umweltrecht, 1994, 15-18; J. Bunde Zur ökonomischen Effizienz des Vorsorgeprinzips in der Umweltpolitik, 1988 mit zahlreichen Beispielen für dogmatisch hinderliche Rückwirkungen des Vorsorgeprinzips. Rechtsvergleichend: M. Germann Das Vorsorgeprinzip als vorverlagerte Gefahrenabwehr, 1993.

${ }^{39} \mathrm{Vgl}$. Mitteilung der Kommission zur Anwendbarkeit des Vorsorgeprinzips, KOM 2000/1 endg., NVwZ Beilage Nr. IV/2001. Danach soll das Vorliegen eines Risikos die Anwendung des Vorsorgeprinzips erst in Gang setzen, so Einleitung, 5. und 5.1. Die Anwendung des Vorsorgeprinzips gehört zum Risikomanagement, das eine Risikobewertung voraussetzt. Das Vorsorgeprinzip wird auf einzelne Maßnahmen bezogen und dadurch gegenständlich eingegrenzt. Vgl. dazu und zu den Unterschieden zum deutschen Verständnis des Vorsorgeprinzips H.-W. Rengeling Bedeutung und Anwendbarkeit des Vorsorgeprinzips im europäischen Umweltrecht, DVBI 2000, 1473, 1478 f.; S. Werner Das Vorsorgeprinzip, UPR 2001, 335, 336-338. Nicht ganz eindeutig ist die Mitteilung der Kommission zur Abgrenzung von Wahrscheinlichkeit und Möglichkeit. Ziff. 5.1 der Mitteilung spricht zwar von „potentiellen Risikos“, rekurriert also auf die Möglichkeit; die weiteren Ziffern, die zur Risikobewertung Stellung nehmen, gehen jedoch von der Wahrscheinlichkeit aus. Einerseits dürfen Vorsorgemaßnahmen nicht zu einem NullRisiko führen (Punkt 6.3.1.), andererseits soll Wahrscheinlichkeit kein Kriterium sein (Punkt 6.2.). Der Kommission gelingt keine sichere Unterscheidung zwischen Möglichkeit und Wahrscheinlichkeit als den Vorsorge-auslösenden Kriterien. Die Tendenz, eher auf die Wahrscheinlichkeit abzustellen, sollte dennoch nicht übersehen werden. In der nicht-deutschen europarechtlichen Literatur wird dem Vorsorgeprinzip ganz überwiegend eine gegenständlich eingeschränkte Bedeutung zugeschrieben; ähnlich ist auch die Deutung des Vorsorgeprinzips im Umweltvölkerrecht, auf die die Kommissions-Mitteilung im Anhang II hinweist. In der deutschen Literatur wird indes gefordert, den Vorsorgegrundsatz getreu dem deutschen Begriffsverständnis zu erweitern, vgl. G. Lübbe-Wolff IVU-Richtlinie und Europäisches Vorsorgeprinzip, NVwZ 1998, 777, 778f. mwN zur nicht-deutschen sowie völkerrechtlichen Literatur; I. Appel Europas Sorge um die Vorsorge, NVwZ 2001, 395, 395, 397, der die zu enge Auslegung des Vorsorgeprinzips durch die Kommission beanstandet, eine weitergehende Auslegung nach deutschem Vorbild fordert und darauf hinweist, dass sich Bedeutung und Inhalt der Vorsorge im Verhältnis 
geprinzip, während in Deutschland mit dem Rückgriff auf das Vorsorgeprinzip ein weiterer Risikobegriff begründet wird. Deutscher und europarechtlicher Risikobegriff beziehen sich daher auf Unterschiedliches: Möglichkeit oder Wahrscheinlichkeit des Schadenseintritts. Vor dem europarechtlichen Hintergrund ist das deutsche Zweistufenmodell ein Auslaufmodell, so dass die rechtswissenschaftliche Erwartungshaltung in Deutschland auf mehr gerichtet ist, als der europarechtliche Risikobegriff einlösen wird. ${ }^{40}$

\section{Risiko als ungewisse Möglichkeit}

Als Rechtsbegriff umfasst Risiko einen Bereich der Ungewissheit, der zwischen Möglichkeit und Wahrscheinlichkeit changiert. Welches Grundverständnis ist richtig? Muss das deutsche Grundverständnis auf wahrscheinliche Gefahren reduziert werden oder hinkt das Europarecht dem Möglichen hinterher? Die Antwort ist eindeutig: Die Möglichkeit eines Schadenseintritts kann kein Gegenstand rechtlicher Regelungen sein. Ein solches Risikoverständnis erzeugt illusionäre Erwartungen und muss aufgegeben werden.

von europäischer und nationaler Ebene unterscheiden können; M. Herdegen/H.-G. De$\operatorname{derer}$ (Fn. 32), die das deutsche Verständnis des Vorsorgeprinzips auf die Kommissionsmitteilung übertragen. Zum Verhältnis des deutschen und europarechtlichen Vorsorgegrundsatzes siehe auch $M$. Schröder Umweltschutz (Fn. 26), § 9, Rn. 35; J. Falke Aktuelles zum Vorsorgeprinzip und anderen programmatischen Orientierungen im Europäischen Umweltrecht, ZUR 2000, 265, 265f.; A. Epiney Umweltrecht (Fn. 26), 99f.; M. Burgi Das Schutz- und Ursprungsprinzip im europäischen Umweltrecht, NuR 1995, 11, 11 f.; W. Kahl Risikosteuerung (Fn. 16), 1118 Fn. 139; ders. Umweltprinzip und Gemeinschaftsrecht, 1993, 21 f., 161 ff., 216; H.-W. Rengeling Umweltvorsorge und ihre Grenze im EWG-Recht, 1989, $37 \mathrm{ff} ., 53 \mathrm{ff}$.

${ }^{40}$ Hinzuweisen ist schließlich auf den Risikobegriff im internationalen Recht, der im Hormonfleischstreit vor der WTO relevant wurde. Vgl. Entscheidung des Appellate Body der WTO, WT/DS26/AB/R, EuZW 1998, 157, 161 Rz. 186f. Auch hier konfligieren unterschiedliche Vorverständnisse von Risiken, die entweder auf Wahrscheinlichkeits- oder Möglichkeitsurteile bezogen sind. Zu dieser Entscheidung B. Eggers Die Entscheidung des WTO Appellate Body im Hormonfall, EuZW 1998, 147; C. Godt Der Bericht des Appellate Body der WTO zum EG-Einfuhrverbot von Hormonfleisch, EWS 1998, 202; M. M. Slotboom The Hormones Case: An Increased Risk of Illegality of Sanitary and Phytosanitary Measures, Common Market Law Review 36 (1999), 471; V. R. Walker Keeping the WTO from Becoming the „World Trans-Science Organisation“, Cornell International Law Journal 31 (1998), 251; $R$. Howse Democracy, Science, and Free Trade: Risk Regulation on Trial at the World Trade Organization, Michigan Law Review 98 (2000), 2329. 


\section{Verhalten statt Technik als Regelungsgegenstand}

Gegenstand des Rechts kann nicht die Technik selbst, sondern nur der Umgang des Menschen mit ihr sein. Denn der Regelungsadressat des Rechts ist immer das Individuum und nicht Natur oder Technik. Wollte das Recht die Möglichkeit von Gefahren ausschließen, müsste es die Möglichkeit freien menschlichen Verhaltens ausschließen. ${ }^{41}$ In letzter Konsequenz bedeutete dies ein Forschungs- und Anwendungsverbot. Doch dieses Mittel wäre zum Ausschluss möglicher Gefahren ungeeignet, denn ob sich die Normadressaten tatsächlich an die normative Erwartung halten, obliegt der freien Entscheidung jedes Einzelnen. Jedes normgemäße Verhalten ist ein individueller Entschluss, auf den das Recht Einfluss nimmt, den es aber nicht ersetzt. Recht muss immer mit normabweichendem Verhalten rechnen. Dieses ist überhaupt die Voraussetzung, Verhalten rechtlich zu regeln, denn andernfalls genügten Sitte und Moral. Normabweichendes Verhalten ist eine theoretische Funktionsbedingung des Rechts. Sein Umfang aber muss verschwiegen bleiben, weil Recht sonst seine Wirksamkeit verlöre. ${ }^{42}$

Daraus folgt: Wenn das Recht einerseits Risikoentscheidungen regeln will und dies nur über die Regelung menschlichen Verhaltens kann, andererseits Menschen in ihrem Entschluss zu normkonformem Verhalten frei und letztlich unberechenbar sind, muss eine auf die tatsächliche Unterbindung der Schadensmöglichkeit ausgerichtete Verhaltensnorm ihrem Anspruch nach scheitern. Sie erwartet einen Zustand der Gefahrlosigkeit, der mit den Mitteln des Rechts nicht zu erzielen ist. Der Anspruch der Risikosteuerung kann sich daher nicht auf gefahrlose $\mathrm{Zu}-$ stände richten, denn diese sind schon aufgrund des sich frei verhaltenden Normunterworfenen nicht zu garantieren. Diese Paradoxie scheint der weit verstandene Risikobegriff überwinden zu können. ${ }^{43}$ In Wirklichkeit scheitert er jedoch an den kognitiven Voraussetzungen jeder Verhaltensregelung.

\footnotetext{
${ }^{41}$ Daher hat eine Leitvorstellung, die das Recht an vermeintlich „objektiven“ technischen oder naturwissenschaftlichen Wirkungszusammenhängen misst, unweigerlich ein unfreiheitliches Menschenbild. Denn als frei gilt nur, was nicht dem Gesetz der Kausalität unterworfen ist, vgl. H. Kelsen Reine Rechtslehre, 2. Aufl. 1960, 95-102 zur Dichotomie von (unfreiheitlicher) Naturgesetzlichkeit und (freiheitlicher) Normzurechnung.

42 Siehe H. Popitz Über die Präventivwirkung des Nichtwissens, 1968, 9, 18, 20: Eine Gesellschaft, die jede Verhaltensabweichung aufdeckte, würde zugleich die Geltung ihrer Normen ruinieren. Ebenso ders. Die normative Konstruktion der Gesellschaft, 1980, 67. Aus juristischer Sicht dazu demnächst $C$. Waldhoff Der Verwaltungszwang, A I, mwN.

43 Wie hier indes N. Luhmann Risiko (Fn. 2), 67, 117, 172f., 183.
} 


\section{Subjektive statt objektiver Erkennbarkeit technischer Ungewissheit}

Technischer Wandel ist eine soziale Konstruktion, deshalb ist er politisch regelbar. Gleichwohl wird er immer wieder als neues kognitives Problem empfunden, weil man meint, dass ein theoretisches Wissen über technische Zusammenhänge jenseits praktischer Erfahrung möglich sei. ${ }^{44}$ Vor der rechtlichen Regelbarkeit liege gewissermaßen der Bereich objektiver technischer Prozesse, der als Sein wirklichkeitswissenschaftlich erkannt werden müsste. Dies fördert die Überzeugung, Innovationen bedürften aufgrund komplexer Wirkungszusammenhänge neuer branchen- und technikspezifischer Sonderbereichsdogmatiken. ${ }^{45}$ Indes: Alle Wirkungszusammenhänge, ob alt oder neu, bedürfen jedenfalls zum Zwecke menschlicher Erkenntnis der praktischen Anwendung, des Experiments und daher notgedrungen der Erfahrung. ${ }^{46}$ Eine auf die Unterbin-

\footnotetext{
44 Erinnert sei etwa an den technischen Fortschritt in der Industrialisierung ab 1830, der den Glauben an die Fähigkeiten der physikalischen Welterkenntnis gestärkt hat. Wissenschaftsgeschichtlich förderte dies den Siegeszug eines am physikalischen Kausalmodell orientierten Positivismus, der eine naturgesetzliche Erkenntnis als Leitbild aller wissenschaftlichen Erkenntnis ausgab. Die Rechtswissenschaft verarbeitete dies mit den Epochen des Begriffs- und Gesetzespositivismus. Jedenfalls im Staatsrecht wurde dieser Positivismus schon um 1900 durch normative Zurechnungskriterien abgelöst, auch wenn die Epoche des „staatsrechtlichen Positivismus“ - wissenschaftsgeschichtlich nicht korrekt - bis in die Weimarer Republik erstreckt wird. Man kann den Richtungs- und Methodenstreit in der Weimarer Staatsrechtslehre auch als Debatte über die Ablösung der physikalischen Naturgesetzlichkeit durch andere Leitbilder verstehen, vgl. aus der umfangreichen Literatur $O$. Lepsius Erkenntnisgegenstand und Erkenntnisverfahren in den Geisteswissenschaften der Weimarer Republik, Ius Commune XXII (1995), 283 mwN. Im Unterschied zum Staatsrecht hat die Verwaltungsrechtslehre eine entsprechende Grundsatzdiskussion noch nicht geführt.

45 Beispielhaft für das Umweltrecht E. Schmidt-Aßmann Das allgemeine Verwaltungsrecht als Ordnungsidee, 1998, $108 \mathrm{f}$.

${ }^{46}$ Vgl. J. Dewey Die Suche nach Gewißheit, 1998 (1929), 41, 50, 55, 72: Es sei eine falsche Idee anzunehmen, praktisches Handeln sei dem theoretischen Erkennen unterlegen und Sicherheit gebe es nur im objektiv Feststehenden. Siehe auch ders. The Public and Its Problems, 1927, 175: Die Glorifizierung einer reinen Wissenschaft sei die Rationalisierung einer Ausflucht. Sie schaffe einen Fluchtpunkt und drücke sich vor der Verantwortung. K. Popper Naturgesetze und theoretische Systeme, in: H. Albert (Hrsg.) Theorie und Realität, 2. Aufl. 1972 (1949), 43, 56: „Der Gang der Wissenschaft besteht im Probieren, Irrtum und Weiterprobieren. Keine bestimmte Theorie kann als absolut sicher betrachtet werden; jede, auch die am besten bewährte, kann unter Umständen wieder problematisch werden. Keine wissenschaftliche Theorie ist sakrosankt." H. Albert Traktat über rationale Praxis, 1978: Wer die Wirklichkeit ganz erkennen will, sieht am Ende nichts mehr. Vgl. auch R. Münch Risikopolitik, 1996, 238: Den Wahrheitsgehalt theoretischer Modelle mit ihrer ungetrübten Verwirklichung in der konkreten Gesellschaft zu verwechseln, sei ein immer wieder gemachter Fehler.
} 
dung möglicherweise gefährlicher Techniken gerichtete Risikodogmatik setzt sich daher ein vergebliches Ziel. Sie hofft auf nicht erreichbares Wissen und wird das Unwissen nie beheben. Indizien sind nötig, keine Hypothesen.

Technik ist kein objektiver Zustand, sondern Ergebnis subjektiver Handlungen. ${ }^{47}$ Thre Wirkungszusammenhänge können nur durch Handlungserfahrung aufgedeckt werden. ${ }^{48}$ Das heißt aber: Schon im Augenblick der ersten Erfahrung mutiert die Möglichkeit zur Wahrscheinlichkeit. Die Schadensmöglichkeit ist daher kognitiv immer nur als Problem der Wahrscheinlichkeit erkennbar. Dann aber sollte kein objektiv gefahrloser Zustand rechtlich bezweckt werden.

Die gerade in Deutschland verbreitete Erwartung, mit einer Risikovorsorge schon die Möglichkeit von Schäden zu vermeiden, ${ }^{49}$ ist auf illusionäre Ziele gerichtet und beruht auf rechtlich nicht umsetzbaren Voraussetzungen. ${ }^{50}$ Juristen geben sich hier als die letzten Vertreter zu erkennen,

\footnotetext{
${ }^{47}$ Daher sollte Technik nicht als objektiver Zustand, sondern als subjektives Handeln begriffen werden, so H. Popitz Technisches Handeln, in: ders. Phänomene der Macht, 1986, 107: „,Technik' meint etwas (von diesem Vorverständnis können wir wohl ausgehen), was der Mensch macht, ins Werk setzt - im Unterschied zu dem, was ohne Zutun des Menschen geworden, ohne sein Zutun gewachsen ist." Zum technischen Handeln als besonders konstruktivem Typus menschlichen Handelns ebd., 115-125. Vgl. auch P. Janich Grenzen der Naturwissenschaft. Erkennen als Handeln, 1992.

${ }^{48} \mathrm{Vgl}$. aus ingenieurwissenschafticher Sicht $M$. Heymann/U. Wengeroth Die Bedeutung von ,tacit knowlegde“ bei der Gestaltung der Technik, in: U. Beck/W. Bon B (Hrsg.) Die Modernisierung der Moderne, 2001, 106, 106, 116ff. zum hohen Stellenwert von praktischem Erfahrungswissen gegenüber einer allein wissenschaftsbasierten Technik. Ähnlich C. Law/S. Böschen Möglichkeiten und Grenzen der Wissensfolgenabschätzung, ebd., 122, 122f., 129f.: Normative Entscheidungen und die Setzung von Prioritäten als Voraussetzungen der Folgenabschätzung.

${ }^{49}$ Vgl. Nachweise Fn. 12, 14, 16, 38.

${ }^{50}$ Vgl. U. Di Fabio Risikoentscheidungen (Fn. 2), 460: „Die Veränderung des Gefahrentatbestandes hin zu Möglichkeitsprognosen, die Absenkung von erwartbaren auf theoretisch denkbare Schäden, die zum Teil nur noch als Auswirkungen verstanden werden, die Wissenschafts-, Vergleichs- und Abwägungsabhängigkeit sowie die Dynamik von Risikoentscheidungen werfen die Frage auf, ob nicht das juristische System mit seiner herkömmlichen Dogmatik die Grenzen seiner Definitionsmacht erreicht hat, "Nach $R$. WolfZur Antiquiertheit des Rechts in der Risikogesellschaft, Leviathan 1987, 357, 385, hat Sicherheit keine reale gesellschaftliche Basis mehr und taugt nicht als konkrete Utopie: „Als politisches Programm ist das Ziel der Sicherheit die Beschwörung vergangener Zeiten." Bedenkenswert auch die Einschätzung des deutschen Umweltrechts durch eine amerikanische Beobachterin: S. Rose-Ackerman Umweltrecht und -politik in den Vereinigten Staaten und der Bundesrepublik Deutschland, 1995, 242, ähnlich 267: „Die weitverbreitete Akzeptanz, die die naturwissenschaftliche Methode und gerichtsförmliche Verfahren finden, legen es natürlich nahe, sie auch auf Problembereiche anzuwenden, für deren Lösung sie nicht geschaffen sind. Im allgemeinen gelingt es dem deutschen Ver-
} 
die der Natur eine apriorische Objektivität zubilligen und glauben, das Verständnis der Natur sei ohne subjektive Konstruktionsleistung möglich. ${ }^{51}$ Der Risikobegriff ist eine moderne Emanation des klassischen erkenntnistheoretischen Problems, technische Realität und naturwissenschaftliche Kausalität als objektives Sein dem Sollen überzuordnen ${ }^{52}$ und dadurch praktisches Handeln zu lähmen. ${ }^{53}$

waltungsrecht daher nur in geringem Maße, Verfahren und materiellen Regelungsgegenstand zur Lösung politisch umstrittener Fragen aufeinander abzustimmen." - Hier wird nur auf die deskriptive Analyse, nicht aber den von Rose-Ackerman im Übrigen aufgezeigten Lösungsweg Bezug genommen.

5I Vgl. statt dessen H. Rickert Die Grenzen der naturwissenschaftlichen Begriffsbildung, 1896; ders. Kulturwissenschaft und Naturwissenschaft, 6. Aufl. 1926; E. Cassirer Substanzbegriff und Funktionsbegriff, 1910; J. Dewey Experience and Nature, 1925, Kap. IV, VI; ders. Gewißheit (Fn. 46); H. Kelsen Vergeltung und Kausalität, 1941; K. Popper Naturgesetze (Fn. 46), $43 \mathrm{ff}$; P. Janich Naturgeschichte und Naturgesetz, in: O. Schwemmer (Hrsg.) Über Natur. Philosophische Beiträge zum Naturverständnis, 1987, 105; U. Beck Gegengifte, 1988, 62ff.; D. v. d. Pfordten Ökologische Ethik. Zur Rechtfertigung menschlichen Verhaltens gegenüber der Natur, 1996, 126-132; B. Latour Das Parlament der Dinge, 2001, $22 \mathrm{ff}$, 41 ff.; J. Nida-Rümelin Die ökologische Herausforderung der Ethik, in: ders./D. v. d. Pfordten (Hrsg.) Ökologische Ethik und Rechtstheorie, 2. Aufl. 2002, 19, 24f.; Akademie der Wissenschaften zu Berlin Umweltstandards, 1992, 24 (naturalistisches Verständnis der Umwelt durch ein kulturalistisches ersetzen).

$52 \mathrm{Vgl}$. die Erklärung für die Objektivierung der Technik durch H. Popitz Technisches Handeln (Fn. 47), 107, 120: Aus der Fähigkeit zum Herstellen entstehe ein Artefakt, das dem handelnden Menschen als etwas Gegenständliches gegenübertrete und als Objekt die Abhängigkeit vom technischen Handeln zu verlieren scheine. Dewey macht im Übrigen der deutschen Philosophietradition den Vorwurf, dass sie die theoretische Abstraktion über die empirische Praxis stelle und dadurch sinnvolles Handeln an nicht erfülbaren Maßstäben messe, vgl. J. Dewey Deutsche Philosophie und deutsche Politik, 2000, 37 ff. (1915/1942). Der Text ist unter dem Eindruck der Kriege entstanden, enthält trotzdem viel Nachdenkenswertes. Zur philosophischen Tradition der Technikkritik in Deutschland und Frankreich S. Fohler, Techniktheorien. Der Platz der Dinge in der Welt des Menschen, 2003. Sie unterscheidet drei Modelle: Ein Fortschrittsmodell, in dem sich der Mensch der Technik bemächtigt, ein Krisenmodell, in dem Technik im Begriff ist, die Kontrolle über die Gesellschaft zu übernehmen und ein drittes Modell, das die Konfrontation von Mensch und Technik durch technische Spielräume ersetzt.

${ }^{53}$ Hinter der bloß theoretischen Sicherheit solcher „objektiven Erkenntnis“ muss die Gewissheit im praktischen Handeln immer zurückbleiben. Jenseits praktischer menschlicher Anwendung ist Wissen über technische Zusammenhänge nicht denkbar. Vgl. dazu J. Dewey Gewißheit (Fn. 46), 10f., 25-30, 40, 74, 212: Die Vorstellung einer theoretisch erreichbaren Sicherheit habe bei der Bevorzugung des Erkennens vor dem Handeln eine entscheidende Rolle gespielt, denn das Denken kenne keine Unsicherheiten, das Handeln hingegen schon. Probleme entstünden, wenn zwischen Theorie und Praxis getrennt werde, denn die Idee, dass das Denken vollständige theoretische Sicherheit liefern könne, lähme das Handeln. Die Erfahrung des Handelns könne aber nicht mehr als eine kontingente Wahrscheinlichkeit liefern. Dewey verknüpft dieses Erfahrungswissen über eine 


\section{Experimente und flexibles Erfahrungswissen}

Die erwartete Vermeidung von Risiken behinderte im Übrigen den Lernprozess mit neuen technischen Mitteln. ${ }^{54}$ Nur im Umgang mit Risiken kann man lernen, sie zu bewältigen. ${ }^{55}$ Erst das Experimentieren mit neuen Techniken liefert das nötige Wissen für eine Risikobewertung. Durch Ausschluss möglicher Risiken Sicherheit zu erstreben, ist daher weder theoretisch noch praktisch sinnvoll. ${ }^{56}$ Am Experiment führt kein Weg vorbei. Rechtliche Regelungen müssen einerseits Experimente zulassen, aus ihnen andererseits aber auch Erfahrungen ziehen, ${ }^{57}$ also individuelle Verhaltensregelungen treffen und sie als Normen verallgemeinern. Das ist aber kein ungewöhnlicher Zielkonflikt, der nur bei technischer oder zeitlicher Komplexität entstünde, sondern eine auch sonst täglich zu treffende Entscheidung. Dass solche Entscheidungen für komplex gehalten werden, ist der natürlichen Vielfalt subjektiver Wahrnehmungen

pragmatische Handlungstheorie mit der Demokratie, die zum einen den institutionellen Rahmen für die Erfahrung bereitstellt und zu testende Hypothesen entwickelt, und zum anderen ihre Ergebnisse aufnimmt und umsetzt; ders. The Public (Fn. 46), 174ff., $202 \mathrm{ff}$. Zur Verknüpfung von Demokratie und Erkenntnistheorie bei Dewey: $R$. Westbrook John Dewey and American Democracy, 1991; M. Kettner John Deweys demokratische Experimentiergemeinschaft, in: $\mathrm{H}$. Brunkhorst (Hrsg.) Demokratischer Experimentalismus, 1998, 44, 53 ff.; F. Jaeger Amerikanischer Liberalismus, 2001, 266 ff., 283 ff.; $R$. Posner Law, Pragmatism and Democracy, 2003, 97-129.

${ }^{54}$ Vgl. dazu K. Heilmann Das Risiko der Sicherheit, 2002, der auf Fehleinschätzungen von Risiken hinweist, die auch auf zu großem Sicherheitsvertrauen beruhen, den Erkenntniswert von Erfahrung und Handeln also unterschätzen. Ähnlich A. Wildavsky Searching for Safety, 1988, $48 \mathrm{ff}$., $205 \mathrm{ff}$.

$55 \mathrm{Vgl}$. dazu vor allem den Ansatz von A. Wildavsky, der beständig darauf hinweist, dass Risikovermeidung den Innovationsprozess ausschließt und daher selbst riskant ist: A. Wildavsky (Fn. 54), insbes. $17 \mathrm{ff}$., 189ff.; ders. But Is It True? A Citizen's Guide to Environmental Health and Safety Issues, 1995; ders. No Risk Is the Highest Risk of All, American Scientist 67 (1979), 32. Wildavysky lehnt das Vorsorgeprinzip ab, instruktiv ders. in: But Is It true, $427 \mathrm{ff}$. Auseinandersetzung mit Wildavsky bei $D$. Kerwer Mehr Sicherheit durch Risiko?, in: P. Hiller/G. Krücken (Hrsg.) Risiko und Regulierung, 1997, 253.

${ }^{56}$ Aus soziologischer Sicht H. Popitz Technisches Handeln (Fn. 47), 128.

${ }^{57}$ Die Bedeutung des „Erfahrungswissens" nimmt zu, während die Bedeutung der rein technischen Rationalität abnimmt, vgl. F. Böhle/A. Bolte/I. Drexel/S. Weishaupt Grenzen wissenschaftlich-technischer Rationalität und „anderes Wissen“, in: Modernisierung der Moderne (Fn. 48), 96, 100f., 103f.; C. Lau/S. Böschen (Fn. 48), 129-135: Erfahrungswissen durch Eingehen von Risiken. Zur juristischen Umsetzung von Erfahrungswissen; W. Berg Vom Wettlauf zwischen Recht und Technik, JZ 1985, 401, $403 \mathrm{ff}$.; F. Nicklisch Das Recht im Umgang mit dem Ungewissen in Wissenschaft und Technik, NJW 1986, 2287, 2288f.; A. Voßkuhle Beteiligung Privater an der Wahrnehmung öffentlicher Aufgaben und staatliche Verantwortung, VVDStRL 62 (2003), 266, 325f. 
geschuldet. Komplexität ist folglich eine Ausgangsbedingung demokratischer Rechtsetzung und nicht Ausdruck einer Zivilisationskrise. ${ }^{58}$

\section{Die Entindividualisierung der Zurechnung}

Warum setzt sich das Recht unerfüllbare Aufgaben zum Ziel? Was ist das Motiv für diese Gegenstandserweiterung? Hier kommen wir auf die eingangs erwähnte Veränderung der juristischen Zurechnungskategorien zurück. Die Ausdehnung auf die Möglichkeit basiert auf zwei Prämissen, die als Naturalisierung und Vergesellschaftung der Zurechnung bezeichnet werden können.

\section{Risiko als Naturalisierung und Vergesellschafiung der Zurechnung}

Technikfolgen werden naturalisiert, nicht individualisiert. Prägend war hier die Erfahrung mit der Kernenergie..$^{59}$ Objektive Schadensmöglich-

$58 \mathrm{~J}$. Madison Federalist Nr. 10, 37, 57, in: A. Hamilton/J. Madison/J. Jay Die Federalist Papers, B. Zehnpfennig (Hrsg.), 1993 (1787/88); H. Kelsen Allgemeine Staatslehre, 1925, 326, 344, 369f.; J. Dewey Freedom and Culture, 1989, 51, 56 (1939); K. Hesse Die verfassungsrechtliche Stellung der politischen Parteien, VVDStRL 17 (1959), 11, 18-20; A. Greifeld Volksentscheid durch Parlamente, 1983, $24 \mathrm{ff}$., $37 \mathrm{ff}$.; F. Hufen Gesetzesgestaltung und Gesetzesanwendung im Leistungsrecht, VVDStRL 47 (1989), 142, 144, 147; C. S. Nino The Constitution of Deliberative Democracy, 1996, $107 \mathrm{ff} ., 134 \mathrm{ff} . ;$ H. Hofmann Das Recht des Rechts, das Recht der Herrschaft und die Einheit der Verfassung, 1998, $23 \mathrm{ff} ., 47 \mathrm{f}$., 55; C. Möllers Theorie, Praxis und Interdisziplinarität in der Verwaltungsrechtswissenschaft, VerwArch 93 (2002), 22, 28 f.

59 Ihre Bewertung hat sich von der staatlich geförderten umweltfreundlichen Lösung des Energieproblems zum staatlich unterbundenen umweltgefährdenden Langzeitrisiko gewandelt, vgl. $R$. Steinberg Die energiewirtschaftliche Nutzung der Kernenergie - Aufstieg und Ausstieg, in: Technikumsteuerung (Fn. 23), 139; U. Di Fabio Der Ausstieg aus der wirtschaftlichen Nutzung der Kernenergie, 1999; M. Schmidt-Preuß Konsens und Dissens in der Energiepolitik, NJW 1995, 985; A. Roßnagel Sicherheitsphilosophien im Technikrecht - am Beispiel des Atomrechts, UPR 1993, 129; J. Radkau Aufstieg und Krise der deutschen Atomwirtschaft, 1983; H. Hofmann Rechtsfragen der atomaren Entsorgung, 1980, insbes. $258 \mathrm{ff}$. Zu weiterreichenden Auswirkungen D. Grimm Die Aufgabenverteilung zwischen Justiz und Verwaltung bei der Genehmigung kerntechnischer Anlagen, in: S. van Buiren/E. Ballerstedt/D. Grimm, Richterliches Handeln und technisches Risiko, 1982, 25; P. Lerche Kernkraft und rechtlicher Wandel, 1981. - In der Soziologie führte die Erfahrung des Reaktorunfalls von Tschernobyl zur Prägung des Begriffs der "Risikogesellschaft", vgl. U. Beck Der anthropologische Schock. Tschernobyl und die Konturen der Risikogesellschaft, in: ders. (Hrsg.) Politik in der Risikogesellschaft, 1991, 67 (1986); schließlich J. Elster Risiko, Ungewißheit und Kernkraft, in: Risiko und Gesellschaft (Fn. 7), 59. 
keiten sollen aufgeklärt werden, bevor Subjekten Schadenswahrscheinlichkeiten zugemutet werden. Die Zurechnung des Schadens wird naturalisiert, weil sie auf naturwissenschaftliche Wirkungszusammenhänge abstellt, die individuellen Handlungen vorausliegen sollen.

Hinzu tritt folgendes. Die diffusen Ursachenzusammenhänge von Umweltrisiken, die unter anderem zeitversetzte, kumulierende und grenzüberschreitende Effekte auslösen, sind ein gesellschaftliches Phänomen, zu dem alle beitragen. ${ }^{60}$ Für schlechte Luft ist letztlich die Gesellschaft verantwortlich. Wird die Zurechnung aber naturalisiert oder vergesellschaftet, muss ein akteurszentriertes Präventionsmodell in der Theorie zu kurz greifen. Die Zurechnung von Ungewissheit verändert sich: von der subjektiven zur objektiven Beherrschbarkeit, vom Einzelnen zum Kollektiv. ${ }^{61}$

60 Darauf gründet die oft artikulierte Sorge, das Recht leide an einer Kausalitätskrise, weil der Mensch kognitiv unfähig sei, die komplexen naturwissenschaftlichen und sozialen Zusammenhänge überhaupt erfassen zu können. Dazu E.-H. Ritter Das Recht als Steuerungsmedium im kooperativen Staat, in: D. Grimm (Hrsg.) Wachsende Staatsaufgaben - sinkende Steuerungsfähigkeit des Rechts, 1990, 69, 72, 85; U. K. Preuß Risikovorsorge (Fn. 6), 526-532; U. Di Fabio Risikoentscheidungen (Fn. 2), $67 \mathrm{ff} ., 88 \mathrm{ff}$;; G. Teubner Die unsichtbare „Cupola“: Kausalitätskrise und kollektive Zurechnung, in: W. Lübbe (Hrsg.) Kausalität und Zurechnung, 1994, 91, 93f.; K.-H. Ladeur (Fn. 2), 15 ff., $69 \mathrm{ff}$., $84 \mathrm{ff}$., $118 \mathrm{ff}$. Die Idee einer „Kausalitätskrise“ beruht indes auf einer fälschlich unterstellten Vergleichbarkeit normativer Zurechnungen mit naturwissenschaftlicher Kausalität. Dies hat erhebliche Auswirkungen auf die juristische Debatte; diese aufzeigend und kritisierend O. Lepsius Steuerungsdiskussion, Systemtheorie und Parlamentarismuskritik, 1999, 31-34; zum Zusammenhang von Entsubjektivierung und Kausalitätskrise ders. Besitz und Sachherrschaft im öffentlichen Recht, 2002, $449 \mathrm{ff}$., $481 \mathrm{ff}$. Vgl. auch G. Vobruba Prävention durch Selbstkontrolle, in: Mensch als Risiko (Fn. 2), 29, 33, 40f., der herausarbeitet, wie die Aktivierung des Bezugs des Subjekts zum System und die Reduktion der Subjekte auf systemische Funktionselemente Krisenvorstellungen auslöst. Zum Problem in einer früheren Sicht A. Kaufmann Tatbestandsmäßigkeit und Verursachung im Contergan-Verfahren, JZ 1971, 569.

61 Wie hier Risiko als Zurechnungsmodus begreifend U. Di Fabio Risikoentscheidungen (Fn. 2), 56, markante Bewertung ebd., 450: „Der maßgebliche Grund für den immer stärker wahrgenommenen Wandlungsprozeß in der Dogmatik des öffentlichen Rechts ist der Übergang von im Prinzip konkreter, individualisierbarer, begrifflich bestimmbarer Gefahrenabwehr zu einer sozialgestaltenden Risikovorsorge." Vgl. auch E. Denninger Der Präventions-Staat, KJ 1988, 1, 1, 3 zu Akzentverlagerungen von einem System der Rechtssicherheit zu einem System der Rechtsgütersicherheit; G. Winter Das Recht der Risikogesellschaft, KJ 1998, 518, 519f.: Trennung in ein wirtschaftliches und ein technisches Syndrom bei Risiken. Zur gesellschaftlichen Zurechnung von Risiken $U$. Beck Risikogesellschaft, 1986; ders. Einleitung, in: Politik in der Risikogesellschaft (Fn. 59), 15, 26; F. Ewald Der Vorsorgestaat, 1993; W. Bon $\beta$ Vom Risiko. Unsicherheit und Ungewißheit in der Moderne, 1995; J. Schmidt Politische Risikoregulierung als Risikoerzeugung?, in: P. Hiller/G. Krücken (Hrsg.) Risiko und Regulierung, 1997, 279, 281 („Risiken sind Zurechnungsartefakte“). Eine soziologische Ironisierung der soziologischen Risikotheo- 
Mit der naturalisierten oder vergesellschafteten Zurechnung von Risiken wird die Abkehr von einer akteurszentrierten Rechtsordnung in Kauf genommen, was zu erheblichen Folgeproblemen führt: ${ }^{62}$ Einerseits schlägt sich die Naturalisierung innerhalb des Demokratieprinzips nieder, etwa bei der Überantwortung von Entscheidungen an Experten im Glauben, Wirkungszusammenhänge müssten objektiv erkannt werden, weil sie die Voraussetzung und nicht die Folge demokratischer Rechtsetzung sind. Andererseits wirkt sich die Vergesellschaftung beim Grundrechtsschutz aus, der von Individuen auf gesellschaftliche Prozesse und Funktionen ausgedehnt wird. ${ }^{63}$

Die Diskussion um den Risikobegriff hat eine verfassungsrechtliche Dimension, die um die Stellung des handelnden Subjekts in der Rechtsordnung kreist. Die Debatte ist Ausdruck einer kognitiven Verschiebung vom Einzelnen auf die Gesellschaft. Nur dann besteht überhaupt das Bedürfnis, über die Mittel des Ordnungsrechts hinaus zu gelangen. Die Kollektivierung der Zurechnung beraubt die Gefahrenabwehrdogmatik der Eckpunkte, mit denen sie Verursachung und Rechtsgüter individuell zurechnet. Hierin liegt die Paradoxie des Risikos: Erst die Naturalisierung

rien bei $B$. Kleinwellfonder Der Risikodiskurs. Zur gesellschaftlichen Inszenierung von Risiko, 1996; als systemtheoretische Selbstreflexion M. Heidenescher Die Beobachtung des Risikos. Zur Konstruktion technisch-ökologischer Risiken in Gesellschaft und Politik, $1999,29 \mathrm{ff}$.

${ }^{62} \mathrm{Vgl}$. die Bewertung durch U. Di Fabio Risikoentscheidungen (Fn. 2), 96: „Die Komplexitätszunahme staatlicher Schutzaufgaben im Umwelt-, technischen Sicherheits- und Produktrecht insgesamt ließ die Vorstellung einer durch Parlamentsgesetze im Wesentlichen gesteuerten Verwaltung bald ebenso inadäquat werden wie das traditionelle Konzept individueller Verantwortungszurechnung und daran geknüpfte Handlungs- und Haftungspflichten." Siehe auch W. Hoffmann-Riem Verwaltungsverfahren und Verwaltungsverfahrensgesetz. Einleitende Problemskizze, in: ders./E. Schmidt-Aßmann (Hrsg.) Verwaltungsverfahren und Verwaltungsverfahrensgesetz, 2002, 9, 39: Verwaltungsrechtswissenschaft werde zur Steuerungswissenschaft; $A$. Scherzberg Wissen, Nichtwissen und Ungewißheit im Recht, in: C. Engel/J. Halfmann/M. Schulte (Hrsg.) Wissen - Nichtwissen - Unsicheres Wissen, 2002, 113, 121f., 142f.; R. Wolf Antiquiertheit (Fn. 50), 384-391: veränderter Ordnungsrahmen für regulative Politik, Neuorganisation des Normsetzungsprozesses, überkommene Muster der Zuschreibung von persönlicher Verantwortung werden obsolet, Entwertung der Kategorien der Schuld und Kausalität.

${ }^{63}$ K.-H. Ladeur Die liberale Rechtsordnung und die Institutionalisierung des Zwangs zur Selbsterneuerung der Gesellschaft - Zur Ermöglichung von Lernfähigkeit in der „Gesellschaft der Organisationen“, in: W. Hoffmann-Riem/J.-P. Schneider (Hrsg.) Rechtswissenschaftliche Innovationsforschung, 1998, 40, 51 ff.; ders. AK-GG, 3. Aufl. 2001, Art. 19 Abs. 3 Rn. 1-4, 18 ff. Die rechtlichen Folgeprobleme diagnostiziert $R$. Wolf Die Risiken des Risikorechts, in: A. Bora (Hrsg.) Rechtliches Risikomanagement, 1999, 65. Er sieht jedoch keinen Ausweg, weil (oder sollte man sagen: solange?) die Rechtsordnung ein umfassendes Sicherheitsversprechen gibt. 
und Vergesellschaftung machen es zu einem Rechtsproblem, das aber gerade wegen der neuen Zurechnungen vom Rechtssystem nicht mehr bewältigt werden kann. Denn weder eine scheinbar objektive Größe wie Natur noch eine kollektive Größe wie Gesellschaft kann ein positiver Zurechnungspunkt des Rechts sein. Rechte und Pflichten treffen nur Rechtssubjekte, hinter denen Individuen stehen. Von Individuen geht die Legitimation der Rechtsordnung aus, zu ihrem Schutz wird sie begrenzt. Gesellschaftliche Auswirkungen sind nur der mittelbare, kumulative Effekt der Handlungszentrierung des Rechts. ${ }^{64}$ Eine gesellschaftliche $\mathrm{Zu}$ rechnung hingegen würde die freiheitssichernde Differenz zwischen der Rechtsordnung, dem Staat, und der Gesellschaft beseitigen.

\section{Rezeption sozialwissenschaftlicher Problemwahrnehmungen}

Die Begriffe „Risiko"65 und „Steuerung"66 kanalisieren das Problem in eine sozialwissenschaftliche Perspektive. ${ }^{67}$ Sie sind terminologische Transmissionsriemen, mit denen technische und gesellschaftliche Erwartungshaltungen disziplinenübergreifend gebündelt werden. Dabei ent-

${ }^{64}$ Vgl. H. Kelsen Der soziologische und der juristische Staatsbegriff, 1922, 34f., 44f.; ders. Reine Rechtslehre, 1. Aufl. 1934, 35, 52-59.

${ }^{65} \mathrm{Vgl}$. G. Bechmann Risiko als Schlüsselkategorie in der Gesellschaftstheorie, in: Risiko und Gesellschaft (Fn. 7), 237, 239 ff., sowie die Nachweise Fn. 2.

${ }^{66}$ Der Begriff der „Steuerung“ und seine Bestandteile „direkte“ und „,indirekte“ Steuerung sind in der verwaltungsrechtlichen Diskussion nahezu omnipräsent geworden, vgl. etwa E. Schmidt-Aßmann/W. Hoffmann-Riem (Hrsg.) Verwaltungsorganisationsrecht als Steuerungsressource, 1997; M. Kloepfer Technikumsteuerung (Fn. 23); K. Vieweg (Hrsg.) Techniksteuerung und Recht, 2000; W. Hoffmann-Riem (Hrsg.) Innovation und Telekommunikation. Rechtliche Steuerung von Innovationsprozessen in der Telekommunikation, 2000; ders. Innovationssteuerung durch die Verwaltung, Die Verwaltung 33 (2000), 155; M. Schmidt-Preuß Steuerung durch Organisation, DÖV 2001, 45, $47 \mathrm{ff}$;; Regulierte Selbstregulierung als Steuerungskonzept des Gewährleistungsstaates, Die Verwaltung Beiheft 4 (2001).

${ }^{67}$ Zum sozialwissenschaftlichen Hintergrund des Begriffs und seiner juristischen Rezeption: $R$. Voigt Grenzen rechtlicher Steuerung, in: ders. (Hrsg.) Recht als Instrument der Politik, 1986, 14; E.-H. Ritter Das Recht als Steuerungsmedium im kooperativen Staat, in: D. Grimm (Hrsg.) Wachsende Staatsaufgaben (Fn. 60), 69; G. F. Schuppert Verwaltungswissenschaft als Steuerungswissenschaft, in: W. Hoffmann-Riem/E. SchmidtAßmann/G. F. Schuppert (Hrsg.) Reform des Allgemeinen Verwaltungsrechts, 1993, 65; U. Di Fabio Risikosteuerung im öffentlichen Recht, in: W. Hoffmann-Riem/E. SchmidtAßmann (Hrsg.) Öffentliches Recht und Privatrecht als wechselseitige Auffangordnungen, 1996, 143, 143-146; G. Roellecke Verwaltung und Verwaltungsrecht, Die Verwaltung 29 (1996), 1, 13f.; E. Schmidt-Aßmann Ordnungsidee (Fn. 45), 18ff., 175ff.; A.Voßkuhle Die Reform des Verwaltungsrechts als Projekt der Wissenschaft, Die Verwaltung 32 (1999), 545, 547-550. 
stammen beide Aspekte, Risiko und Steuerung, unterschiedlichen Theorieansätzen innerhalb der Soziologie: der Idee der Risikogesellschaft und der Idee der Steuerungswissenschaft. Ihre juristische Symbiose beleuchtet das Problem der interdisziplinären Transferleistung, ${ }^{68}$ das bei der Umsetzung sozialwissenschaftlicher Diagnosen zu bewältigen ist. ${ }^{69}$ Auch unter dem Eindruck einer sozialwissenschaftlichen Öffnung der Rechtswissenschaft ${ }^{70}$ sollten solche Perspektivenimporte nicht zu einem kogni-

${ }^{68}$ Zur „interdisziplinären Transferleistung “ C. Möllers Theorie und Praxis (Fn. 58), $34 \mathrm{ff}$; $H$. Schulze-Fielitz Was macht die Qualität öffentlich-rechtlicher Forschung aus?, JöR 50 (2002), 1, 50-52; A. Voßkuhle Schlüsselbegriffe der Verwaltungsrechtsreform, VerwArch 92 (2001), 184, 196f., 209f.; ders. Methode und Pragmatik im Öffentlichen Recht, in: H. Bauer u.a. (Hrsg.) Umwelt, Wirtschaft und Recht, 2002, 171, 181 ff.; exemplarisch am Begriff der „Verantwortung“ H. C. Röhl Verwaltungsverantwortung als dogmatischer Begriff?, Die Verwaltung Beiheft 2 (1999), 33, 34f., 46f., $53 \mathrm{ff}$. Eine Paralleldebatte wird bezogen auf die Ökonomik geführt, vgl. nur $M$. Morlok Vom Reiz und vom Nutzen, von den Schwierigkeiten und den Gefahren der Ökonomischen Theorie für das Öffentliche Recht, in: C. Engel/ders. (Hrsg.) Öffentliches Recht als ein Gegenstand ökonomischer Forschung, 1998, 1, 10ff. und die weiteren Beiträge dieses Bandes; O. Lepsius Die Ökonomik als neue Referenzwissenschaft für die Staatsrechtslehre?, Die Verwaltung 32 (1999), 429; J.-P. Schneider Zur Ökonomisierung von Verwaltungsrecht und Verwaltungswissenschaft, Die Verwaltung 34 (2001), 317; M. Wallerath Die Ökonomisierung des Staates, JZ 2001, 209.

69 Bei der Diskussion um den Risikobegriff hat man den Eindruck, als ob die beiden unterschiedlichen soziologischen Risikoverständnisse in der juristischen Diskussion durchkreuzt werden. Wer ein steuerungswissenschaftliches Grundverständnis teilt oder systemtheoretisch inspiriert ist, benutzt oft den gesellschaftlichen Risikobegriff der „Theorie der zweiten Moderne" Becks und mißt ihn an systemtheoretisch vorgeprägten rechtlichen Erwartungen. Schon soziologisch könnte diese juristische Gegenüberstellung nicht bewältigt werden. Auch um soziologisch die Kommensurabilität der Diskussionsebenen zu gewährleisten, muss ein auf das Individuum bezogener Risikobegriff verwendet werden. Andernfalls wird eine weder juristisch noch gesellschaftstheoretisch zu bewältigende Kluft nicht auflösbarer Theorieerwartungen konstruiert.

$70 \mathrm{Zu}$ früheren Ansätzen vgl. D. Grimm (Hrsg.) Rechtswissenschaft und Nachbarwissenschaften, Band 1, 2. Aufl. 1976; G. Dilcher/W. Hassemer/W. Hoffmann-Riem/ N. Horn (Hrsg.) Sozialwissenschaften im Studium des Rechts, 4 Bände 1977-1978; W. Hoffmann-Riem (Hrsg.) Sozialwissenschaften im Öffentlichen Recht, 1981. Die empirische Bandbreite der Sozialwissenschaften wird in der juristischen Diskussion nicht ausgeschöpft. Die Rezeption sozialwissenschaftlicher Ergebnisse im deutschen öffentlichen Recht ist theorielastig und empiriefern. Dazu C. Möllers Theorie und Praxis (Fn. 58), 40-42. Zur sehr viel empirischeren Ausrichtung in den USA J. Goldsmith/A. Vermeule Empirical Methodology and Legal Scholarship, University of Chicago Law Review 69 (2002), 153; R. Revesz A Defense of Empirical Legal Scholarship, ebd., 169; R. Erickson/R. Simon The Use of Social Science Data in Supreme Court Decisions, 1998; J. Monahan/L. Walker Social Science in Law, 3. Aufl. 1994; P. Rosen The Supreme Court and Social Science, 1972; sowie die umfangreiche Literatur über den Legal Realism, dazu nur J. Schlegel American Legal Realism and Empirical Social Science, 1995; historisch: 
tiven und dogmatischen Auftrag des Verwaltungsrechts erklärt werden, zumal die gesellschaftstheoretische Problemformulierung des Risikos weder für die Rechtswissenschaft verpflichtend noch in den Sozialwissenschaften unumstritten ist.71

Das gilt auch für den Gebrauch des Begriffs der "Steuerung“. Auch er ist Ausdruck einer kognitiven Veränderung, denn er suggeriert eine rechtliche Beherrschbarkeit individueller Entscheidungen, die schon mit der Grundkonstante des freien Individuums unvereinbar ist. ${ }^{72}$ Wer von "Steuerung“" spricht, hat die Entindividualisierung des Zurechnungssubjekts schon vorausgesetzt. ${ }^{73}$ Der Begriff der Steuerung und erst recht das Leitbild einer Steuerungswissenschaft sollten in juristischen Kontexten vermieden werden, weil sie unangemessene Erwartungshaltungen mitschleppen und den Gegensatz zwischen dem individuellen Verhalten und seinen gesellschaftlichen Reflexen aufheben. ${ }^{74}$

O. Lepsius Verwaltungsrecht unter dem Common Law, 1997, 240ff.; N. Duxbury Patterns of American Jurisprudence, 1995, $65 \mathrm{ff}$.

${ }^{71}$ Zur Kritik in den Sozialwissenschaften $R$. Münch Die „zweite Moderne“: Realität oder Fiktion?, Kölner Zeitschrift für Soziologie und Sozialpsychologie 54 (2002), 417; ders. Risikopolitik, 1996, $34 \mathrm{ff}$; B. Gill Ungewißheit, administrative Entscheidungen und Demokratie, ÖZP 27 (1998), 29, $39 \mathrm{ff}$; K. v. Beyme Der Gesetzgeber, 1997, $19 \mathrm{ff}$.; J. Ale$x a n d e r / P$. Smith Social Science and Salvation: Risk Society as Mythical Discourse, Zeitschrift für Soziologie 25 (1996), 251; D. Brock Die Risikogesellschaft und das Risiko soziologischer Zuspitzung, Zeitschrift für Soziologie 20 (1991), 12; G. Bechmann Risiko als Schlüsselbegriff der Gesellschaftstheorie, KritVj 1991, 212; H. Joas Das Risiko der Gegenwartsdiagnose, Soziologische Revue 11 (1988), 1. Vgl. auch T. Kron (Hrsg.) Individualisierung und soziologische Theorie, 2000; M. Schroer Das Individuum der Gesellschaft, 2000. Zum prinzipiellen Problem H. Albert Theorien in den Sozialwissenschaften, in: ders. (Hrsg.) Theorie und Realität (Fn. 46), $3 \mathrm{ff}$.

72 O. Lepsius Steuerungsdiskussion (Fn. 60), 10ff.; C. Möllers Braucht das öffentliche Recht einen neuen Methoden- und Richtungsstreit?, VerwArch 90 (1999), 187, $203 \mathrm{ff}$.; R. Münch Risikopolitik, 1996, $41 \mathrm{ff}$.

${ }^{73}$ So etwa W. Hoffimann-Riem Organisationsrecht als Steuerungsressource, in: Verwaltungsorganisationsrecht (Fn. 66), 355, 358f., der von der „Beeinflussung von Ergebnisabläufen (Ereignisketten, -kaskaden, -rückkoppelungen u.a.)“ spricht. Ihm zustimmend A. Voßkuhle Methode (Fn. 68), 181.

${ }^{74}$ Kritisch auch C. Möllers (Fn. 72), $197 \mathrm{ff}$.; ders. Theorie und Praxis (Fn. 58) $44 \mathrm{ff}$.; anderer Ansicht E. Schmidt-Aßmann Zur Reform des Allgemeinen Verwaltungsrechts, in: W. Hoffmann-Riem/E. Schmidt-Aßmann/G. F. Schuppert (Fn. 67), 11, 15; G. F. Schuppert Verwaltungswissenschaft (Fn. 67), 65, 69ff.; ders. Verwaltungswissenschaft, 2000, $347 \mathrm{ff}$; ders. Staatswissenschaft, 2003, 90, 389-395, 457-459. 


\section{Rückkehr zu individualisierenden Zurechnungen als soziale Leistung des Rechts}

Nach alledem gilt: Wer Risikosteuerung als juristisches Problem versteht, muss den Regelungsanspruch reduzieren: Von der Ungewissheit der Möglichkeit auf die Ungewissheit der Wahrscheinlichkeit; von einer kollektiven Zuschreibung auf eine subjektive Zurechnung. ${ }^{75}$ Andernfalls würden soziale oder technische Erwartungen geweckt, die das Recht schon theoretisch nicht erfüllen kann und die praktische Enttäuschungen hervorrufen müssen. ${ }^{76}$ Sie schlagen sich dann als Normerosion, ${ }^{77}$ Vollzugsdefizit, ${ }^{78}$

\footnotetext{
75 Die kognitiven Probleme bei der Erkenntnis von Kausalbeziehungen können nur durch ihre normative Transformation in Zurechnungen überwunden werden. Vgl. dazu schon H. Kelsen Kausalität (Fn. 51): In primitiven Gesellschaften herrschten kollektivistische Einstellungen vor (21-23); der Primitive begreife die Natur als ein Stück der Gesellschaft. Individuelle Zurechnungen und normatives Denken sind ein sozialer Fortschritt gegenüber naturalistischen Kausalitätsvorstellungen (46, 43-49, 279-282). Siehe auch ders. Kausalität und Zurechnung, ÖZöR 6 (1954), 125; ders. Reine Rechtslehre, 2. Aufl. 1960, 79-102. Nur im Zurechnungsmodus kann zudem die Willensfreiheit des Menschen bewahrt bleiben; das Kausalmodell muss den Menschen für unfrei halten, vgl. ebd., $95 \mathrm{ff}$.

${ }^{76}$ Die Überforderung des Staates durch übertriebene Erwartungen an Staatsaufgaben beklagen auch $R$. Wahl/I. Appel Prävention (Fn. 11), 30, 67, 213f. Das Phänomen positiv umsetzend H.-J. Koch Innovationssteuerung im Umweltrecht, in: Innovationsforschung (Fn. 63), 273, 275-280. Dass Sicherheit und Unsicherheit keine festen, eindeutig bestimmbaren Größen, sondern veränderbare gesellschaftliche Konstruktionen sind, belegen W. Bonß/J. Hohl/A. Jakob Die Konstruktion von Sicherheit in der reflexiven Moderne, in: Modernisierung der Moderne (Fn. 48), 147, 147 f., $157 \mathrm{f}$.

${ }^{77}$ Beispielhaft $R$. Steinberg Symbolische Umweltpolitik unter besonderer Berücksichtigung der Beschleunigungsgesetzgebung, in: B. Hansjürgens/G. Lübbe-Wolff (Hrsg.) Symbolische Umweltpolitik, 2000, 63, 92-95; im Zusammenhang mit Risikoangst W. Hassemer (Fn. 1), 630.

${ }^{78}$ Vgl. K. Dammann Vollzugsdefizite oder Vollzugsfehler? Juristische Risiken bei Umweltschutz- und Technikkonflikten, in: Risiko und Regulierung (Fn. 55), 39, 55, 61 ff., der erläutert, wie gerade im Umwelt- und Technikrecht Abweichungen von bloßen Erwartungen fälschlich als Vollzugsdefizit problematisiert werden. Aus der umfangreichen juristischen Literatur vor allem $G$. Lübbe-Wolff Erscheinungsformen symbolischen Umweltrechts, in: B. Hansjürgens/G. Lübbe-Wolff (Hrsg.) Symbolische Umweltpolitik, 2000, 25, 28-42; dies. Modernisierung (Fn. 38), 1 ff., 71 ff. mwN; dies. Vollzugsprobleme der Umweltverwaltung, NuR 1993, 217, 220, 226f.; W. Brohm Situative Gesetzesanpassung durch die Verwaltung, NVwZ 1988, 794, 796; aus soziologischer, politik- und wirtschaftswissenschaftlicher Sicht: $R$. Wolf Technik (Fn. 4), $205 \mathrm{ff}$.; A. Windhoff-Héritier Politikimplementation, 1980; J. Hucke Die Implementation umweltpolitischer Programme, in: E. Gawel (Hrsg.) Institutionelle Probleme der Umweltpolitik, 1996, 59; M. van Mark Die Politik- und Vollzugskosten der Umweltpolitik, ebd., 128.
} 
Steuerungsversagen ${ }^{79}$ oder auch Politikverdrossenheit nieder. Gerade weil es sich beim Risiko um ein gesellschaftliches oder technisches Problem handelt, liegt die soziale Leistung des Rechts nicht in einem vermeintlich wirklichkeitsorientierten Auftrag, soziale Komplexität abzubilden, sondern in der Reduktion der Komplexität durch juristische Zurechnungen. ${ }^{80}$ Ungewissheit darf juristisch weder auf die Gesellschaft noch auf eine naturwissenschaftlich objektive Technikbeherrschung bezogen werden, weil diese Ziele juristisch nicht einlösbar sind, so dass das Recht seine soziale Leistung zur Komplexitätsreduktion nicht erbringen könnte.

\section{Risiko als ungewisse Wahrscheinlichkeit}

Die Aufgabe des Risikoverwaltungsrechts kann nur die individuelle Zurechnung ungewisser Wahrscheinlichkeit sein. ${ }^{81}$ Dabei bedarf es zugleich einer Form, die sich vom Zurechnungsmodell der klassischen Gefahrenabwehr unterscheidet.

\footnotetext{
${ }^{79}$ Krisenszenarien etwa bei $R$. Pitschas Verwaltungsverantwortung und Verwaltungsverfahren, 1990, 48-71; G.-P. Calliess, Prozedurales Recht, 1999, 60-83. Vgl. auch D. Grimm Der Wandel der Staatsaufgaben und die Krise des Rechtsstaats, in: Wachsende Staatsaufgaben (Fn. 60), 291, 294-296; T. Ellwein/J. J. Hesse Der überforderte Staat, 1994, $67 \mathrm{ff}$.

${ }^{80} \mathrm{Vgl}$. J. Frank Kollektive oder individuelle Steuerung der Umwelt? KJ 22 (1989), 36, der individualisierende Regelungstechniken empfiehlt und auf Defizite kollektiver Steuerungsansätze aufmerksam macht. Für eine Re-Individualisierung auch C. Lau Neue Risiken und gesellschaftliche Konflikte, in: Politik in der Risikogesellschaft (Fn. 59), 248, 258, 261 f. G. Lübbe-Wolff Modernisierung (Fn. 38), 84f. zur Überkomplexität auf der Ebene der gesetzlichen und verordnungsrechtlichen Vorschriften; 99f., 139ff. zur Komplexitätsreduktion durch Normsetzung; Forderung nach Herstellung von Zurechenbarkeit auch bei $U$. Beck Gegengifte, 1988, 287 (,andere Regeln, andere Wirklichkeit“). Im Umkehrschluss $R$. Wolf Antiquiertheit (Fn. 50), 391: „Je mehr das Recht seine ,Resonanzfähigkeit' für moderne technische Entwicklungen zu sichern versucht, um so mehr hört es auf, normativer Maßstab für das Erlaubte und Gebotene der Technik zu sein.“

${ }^{81}$ Kausalitäten können nur als Wahrscheinlichkeitsgesetze formuliert werden, so schon H. Kelsen Kausalität (Fn. 51), 270, 276. Die Kausalität ist kein objektives Band zwischen Ursache und Wirkung. „Nur eine Norm kann mit dem Anspruch auf Unverbrüchlichkeit auftreten; denn sie ist keine Aussage über die Wirklichkeit und kann daher zu dieser niemals in Widerspruch geraten. Diese Wirklichkeit aber - so wie sie nun einmal menschlicher Erkenntnis gegeben ist - lässt keine unverbrüchliche Gesetzlichkeit als Deutungsschema zu, sie lässt sich nicht durch ein absolute Notwendigkeit des Geschehens behauptendes Kausalgesetz beschreiben." Ebd., 277.
} 


\section{Relations- und Selektionsbedürftigkeit}

Wahrscheinlichkeit kann nicht absolut, sondern immer nur im Verhältnis verschiedener Parameter bestimmt werden. Wir stehen vor einer Relationierungsaufgabe. ${ }^{82}$ Relationen müssen juristisch ermittelt werden, damit Wahrscheinlichkeitsurteile überhaupt getroffen werden können. Sonst ließen sich nur Hypothesen konstruieren, die wieder dem rechtlich nicht regelbaren Bereich der Möglichkeit zuzuordnen wären. Mit Relationierungen wird der Weg aus der Ungewissheitsfalle gewiesen: Das Recht selbst trägt durch Zurechnungen zur Beseitigung faktischer Ungewissheit bei. ${ }^{83}$ Die potentielle Unendlichkeit naturwissenschaftlicher Wirkungszusammenhänge muss selektiert werden, sonst blieben Wirkungszusammenhänge objektiv unendlich und subjektiv nicht erkennbar. ${ }^{84}$ Der Adressat rechtlicher Risikoregulierung ist daher nicht der Mathematiker, sondern der Ingenieur. Er wäre überfordert, wenn er Wahrscheinlichkeitsurteile über nicht relationierte Gefahren abgeben soll. ${ }^{85}$

82 Vgl. J. Dewey Gewißheit (Fn. 46), 107: Relationen bilden die eigentlichen Gegenstände der Wissenschaft. „Die Zurückführung der Erfahrungsgegenstände auf die Form von Relationen, die bezüglich ihrer qualitativen Eigenschaften neutral sind, ist eine Voraussetzung der Fähigkeit, den Verlauf der Veränderungen so zu regulieren, dass er zu dem Vorkommen eines Gegenstandes mit den gewünschten Qualitäten führt." Ähnlich ebd., 128-131, 148, 181. Vgl. auch E. Cassirer Substanzbegriff und Funktionsbegriff, 6. Aufl. 1990 (1910), insbes. $148 \mathrm{ff}$., $359 \mathrm{ff}$., $410 \mathrm{ff}$., der nach einer gründlichen Untersuchung zeitgenössisch moderner naturwissenschaftlicher Begriffsbildungen herausarbeitet, dass die wissenschaftliche Erkenntnis nicht auf Substanzbegriffe, sondern Relationsbegriffe gerichtet ist.

${ }^{83} \mathrm{Vgl}$. dazu $R$. Wahl Risikobewertung (Fn. 13), 279-281, 295, der von ,freiheitssichernden Anforderungen an die Darlegung der Kausalität" (281) spricht. Der Gefahrenbegriff bezeichne die Stelle, bei der es dem Staat erlaubt ist, freiheitsbeschränkende Maßnahmen durchzuführen. Die Bestimmung der Gefahrenschwelle sei eine sehr sensible Stelle im Verhältnis des Staates zum Einzelnen. Ihre Fixierung setze unvermeidlich Wertungen voraus, ebd. 279.

84 Dazu E. Cassirer (Fn. 82), 410-432.

$85 \mathrm{Vgl}$. M. Heymann/U. Wengenroth (Fn. 48), 109: Die Ingenieurwissenschaften hätten sich seit dem Zweiten Weltkrieg immer mehr dem mathematischen Naturwissenschaftsideal angenähert und sich als angewandte Naturwissenschaften begriffen. Dies wird von den Autoren kritisiert. Sie fordern für die Ingenieurwissenschaften die Rückkehr zum Könnensbeweis statt des Wahrheitsbeweises. Ersterer sei in den letzten Jahren auf dem Vormarsch, und der menschliche Faktor in der Konstruktion und das Erfahrungswissen des Konstrukteurs seien im Begriff, wieder entdeckt zu werden (116f.). Die von Experten bestimmte ,richtige Technik“ müsse einer von der Gesellschaft politisch bestimmten gewünschten Technik weichen. Dieses Technikverständnis kreise nicht mehr um den Begriff der Wahrheit, sondern den des Gelingens, ebd. 121. 
An der Relationierungsaufgabe des Rechts führt kein Weg vorbei. ${ }^{86}$ Daher wäre es kontraproduktiv, das Heil in holistischen oder ganzheitlichen Ansätzen zu suchen. ${ }^{87}$ Diese scheuen vor der Selektion in der Annahme zurück, der Komplexität nicht gerecht zu werden, obwohl sich gerade nur durch komplexitätsreduzierende Selektion die Ungewissheit juristisch beheben lässt. ${ }^{88}$

${ }^{86}$ Zur Notwendigkeit von Selektionsentscheidungen und einem sektoralen Regelungszugriff vgl. E. Gawel Umweltpolitik durch gemischten Instrumenteneinsatz, 1991, $53 \mathrm{ff}$; ders. Umweltordnungsrecht - ökonomisch irrational? in: ders./G. Lübbe-Wolff (Hrsg.) Rationale Umweltpolitik - rationales Umweltrecht, 1999, 237, 243; R. Wahl/I. Appel Prävention (Fn. 11), 28f., 58f., $92 \mathrm{ff}$., 118, $125 \mathrm{ff}$,; K.-H. Ladeur Risikobewertung und Risikomanagement im Anlagensicherheitsrecht, UPR 1993, 121, 122 (die Annahme von Wahrscheinlichkeiten ist keine Eigenschaft von Tatsachen, Objekten oder Systemen, sondern beruht auf kognitiven Prämissen); ders. Negative Freiheitsrechte und gesellschaftliche Selbstorganisation, 2000, 75, 99f,; ders. Die rechtswissenschaftliche Methodendiskussion und die Bewältigung des gesellschaftlichen Wandels, RabelsZ 64 (2000), 60, 80; A. Voßkuhle Der Wandel von Verwaltungsrecht und Verwaltungsprozeßrecht in der Informationsgesellschaft, in: W. Hoffmann-Riem/E. Schmidt-ABmann (Hrsg.) Verwaltungsrecht in der Informationsgesellschaft, 2000, 349, 394; O. Lepsius Besitz (Fn. 60), 172 ff., $192 \mathrm{ff}$., $429 \mathrm{ff}$.

${ }^{87}$ Dies befürwortend hingegen W. Hoffmann-Riem Modernisierung von Recht und Justiz, 2001, 67, 93, 201, 363; ders. Organisationsrecht als Steuerungsressource, in: Verwaltungsorganisationsrecht (Fn. 66), 355-395 (376, 385, 395); ders. Verwaltungsrechtsreform - Ansätze am Beispiel des Umweltschutzes, in: ders./E. Schmidt-ABmann/ G. F. Schuppert (Hrsg.) Reform des Allgemeinen Verwaltungsrechts, 1993, 115-175, 167; ders. Ganzheitliche Verfassungsrechtslehre und Grundrechtsdogmatik, AöR 116 (1991), 501-520, 503 Fn. 16, 516ff.; E. Bohne Langfristige Entwicklungstendenzen im Umwelt- und Technikrecht, in: E. Schmidt-ABmann/W. Hoffmann-Riem (Fn. 67), 217, 228-238, 253 f.; K. Lange/A. Karthaus Medienübergreifende Umweltverantwortung, in: K. Lange (Hrsg.) Gesamtverantwortung statt Verantwortungsparzellierung im Umweltrecht, 1997; D. Murswiek Privater Nutzen und Gemeinwohl im Umweltrecht, DVB1 1994, 77, 79; abgeschwächt auch bei E. Schmidt-Aßmann Strukturen des Europäischen Verwaltungsrechts: Einleitende Problemskizze, in: ders./W. Hoffmann-Riem, ebd., 9, 30-32, 39.

88 Zur Kritik ganzheitlicher Ansätze $R$. Breuer Gutachten B, 59. DJT 1992, 115; R. Steinberg Zulassung von Industrieanlagen im deutschen und europäischen Recht, NVwZ 1995, 209, 218; ders. Verfassungsstaat (Fn. 11), 124f.; J. Masing Kritik des integrierten Umweltschutzes, DVBl 1998, 549, 551 f., 554; U. Di Fabio Integratives Umweltrecht, NVwZ 1998, 329, 331, 333, 336; $R$. Wahl Materiell-integrative Anforderungen an die Vorhabenzulassung, NVwZ 2000, 502, 503-506; O. Lepsius Besitz (Fn. 60), 426-430; ders. Vom Abfall zum Produkt, NVwZ 2003, 1182, 1187. 


\section{Normative Nachverdichtung der offenen Relationierung des Gefahrenbegriffs}

Entscheidend ist vielmehr, nach welchen Kriterien die Selektionsentscheidung getroffen wird, denn diese legt die Parameter fest, auf deren Basis Wahrscheinlichkeitsurteile gefällt werden können. Das Grundmuster einer Relationierung enthält das Polizeirecht: Bei hinreichender Wahrscheinlichkeit des Schadenseintritts wird eine Rechtsgutverletzung einer Handlung ${ }^{89}$ zugerechnet. Die Gefahrenabwehrdogmatik lässt alle Parameter offen: Sie kann sich auf jede Handlung beziehen, jede Rechtsgutverletzung erfassen und ist auch in puncto Wahrscheinlichkeit schwer begrenzbar. ${ }^{90}$ Die Offenheit des Gefahrenbegriffs wird durch den faktischen Einzelfallbezug konkretisiert. ${ }^{91}$ Eine Wahrscheinlichkeitsprognose kann erst getroffen werden, wenn im Einzelfall eine Rechtsgutverletzung droht. Erst der konkrete faktische Fallbezug ermöglicht die Relationierung. Daher bedarf bereits eine abstrakte Gefahr der normativen Konkretisierung durch eine Verordnung. Bloße Tatsachen genügen mangels Einzelfallbezugs nicht mehr für die Wahrscheinlichkeit der Rechtsgutverletzung. Die abstrakte Gefahr bedarf der Normierung.

Hier kommt der Risikobegriff zu seinem Recht. Wenn keine faktische Relationierung möglich ist, kann nicht mehr mit dem klassischen Gefahrenbegriff gearbeitet werden. ${ }^{22}$ Gerade weil der offene Gefahrenbegriff

\footnotetext{
89 Oder: Sachherrschaft; zu den damit verbundenen Abgrenzungen und unterschiedlichen Zurechnungsmodi $O$. Lepsius Besitz (Fn. 60), 220ff.

${ }^{90}$ Seit jeher strittig ist, welche Kausalitätserfordernisse an die Wahrscheinlichkeit zu stellen sind und ob sie subjektiv oder objektiv zu ermitteln ist, vgl. F. Schoch Polizei- und Ordnungsrecht, in: E. Schmidt-Aßmann (Hrsg.) Besonderes Verwaltungsrecht, 12. Aufl. 2003, 2. Kap. Rn. 89-92; M. Möstl Die staatliche Garantie für die öffentliche Sicherheit und Ordnung, 2002, 164f.; R. Poscher Gefahrenabwehr, 1999, $49 \mathrm{ff}$., $83 \mathrm{ff}$., 114ff.; E. Denninger in: H. Lisken/E. Denninger (Hrsg.) Handbuch des Polizeirechts, 3. Aufl. 2001, Rn. E $30 \mathrm{ff}$., E 42; C. Gusy Polizeirecht, 5. Aufl. 2003, Rn. 107-120; B. Pieroth/ B. Schlink/M. Kniesel Polizei- und Ordnungsrecht 2002, 65f.; BVerwGE 45, 51, 57.

${ }^{1}$ Zur Leistungsfähigkeit des Gefahrenbegriffs bei stabilen Wissensbeständen und ihren strukturbildenden Paradigmen im Vergleich zum „Risiko“ auch K.-H. Ladeur Umweltrecht (Fn. 2), 78; ders. Risikooffenheit und Zurechnung, in: W. Hoffmann-Riem/ E. Schmidt-Aßmann (Hrsg.) Innovation und Flexibilität des Verwaltungshandelns, 1994, $111,114-121$.

${ }^{22}$ So etwa $U . K$. Preuß Risikovorsorge (Fn. 6), 529f.; K.-H. Ladeur Risikobewertung (Fn. 86), 123f.; ders. Umweltrecht (Fn. 2), 69ff.; A. Scherzberg Risiko als Rechtsproblem, VerwArch 84 (1993), 484, 495-497; U. Di Fabio Risikovorsorge (Fn. 16), 163; ders. Risikoentscheidungen (Fn. 2), 67f.; R. Wahl (Fn. 15) 26. Lfg. 1997, Vorbem. GenTG Rn. 19; G. Lübbe-Wolff IVU-Richtlinie (Fn. 39), 778. Vgl. auch K.-H. Ladeur Risikooffenheit (Fn. 91), 121: Die die kognitive Infrastruktur des Gefahrenbegriffs bildende Erfahrung sei
} 
potentiell auf jede faktische Relation anwendbar ist, ist er auf ungewisse Wahrscheinlichkeiten nicht anwendbar, denn diesen fehlt die Konkretisierbarkeit durch den Einzelfall. ${ }^{93}$ Nicht aus normativen, sondern aus faktischen Gründen genügt der Gefahrenbegriff nicht mehr.

Dieses Defizit darf nun aber nicht dadurch behoben werden, dass naturwissenschaftliche oder sozialtheoretische Zuschreibungen die Funktion des Sachverhaltsbezugs übernehmen. Aus der faktischen Relationsbedürftigkeit im Einzelfall darf nicht auf die normative Relationsunfähigkeit im Allgemeinen geschlossen werden. Risiko als Rechtsproblem vermittelt uns vielmehr die Einsicht, dass die Lösung nicht in faktischen Relationierungen, sondern in der Normativierung der Zurechnungsparameter gefunden werden muss. Mit anderen Worten: Normative Bewertungen müssen die Funktion der faktischen Konkretisierung übernehmen. Dies zu erreichen muss das Ziel der Risikoregulierung sein. Die Vielzahl unbekannter Variablen muss durch normative Selektion und Konkretion reduziert werden. ${ }^{94}$

Die Lösung liegt also nicht in der prinzipiellen Abkehr von der ordnungsrechtlichen Gefahrenabwehrdogmatik, sondern in ihrer normativen Nachverdichtung. Dabei stellen sich zwei Aufgaben: zum einen die materielle Umsetzung durch adäquate Instrumente, zum anderen die organisatorische Umsetzung durch institutionelle Differenzierung.

\footnotetext{
in jüngster Zeit durch normative Elemente und durch Regeln ersetzt worden, die an die Stelle von situativen Abschätzungen getreten seien.

${ }^{93}$ Dies zeigt sich übrigens auch in der Dogmatik des Polizeirechts selbst, dessen Eingriffsvoraussetzungen einerseits reduziert werden, etwa durch geringere Faktenerfordernisse, welche die konkrete Gefahr einer bloßen Gefährlichkeit annähern, andererseits durch zahlreiche neue Tatbestände erweitert werden. Vgl. D. Kugelmann Der polizeiliche Gefahrenbegriff in Gefahr?, DÖV 2003, 781, 783ff.; K. Waechter Zur aktuellen Situation des Polizeirechts, JZ 2002, 854, 855, 857f. Wie das Polizeirecht Elemente des Vorsorgeprinzips dogmatisch verarbeitet verdeutlichen $C$. Möllers Polizeikontrollen ohne Gefahrenverdacht, NVwZ 2000, 382; H.-H. Trute Die Erosion des klassischen Polizeirechts durch die polizeiliche Informationsvorsorge, FS Jeand'heur, 1999, 403, 406-412; D. Neumann Vorsorge und Verhältnismäßigkeit, 1994, 18-30.

${ }_{94}$ Dazu auch K.-H. Ladeur Umweltrecht (Fn. 2), 78, 94-97; R. Wahl Risikobewertung (Fn. 13), 284, der von einer Zumutbarkeitsgrenze spricht, die Wertungen erfordere: „Diese müssen zwar möglichst auf der Grundlage fachwissenschaftlicher Aussagen ergehen, sie enthalten aber zugleich Elemente jenseits der alleinigen fachwissenschaftichen Bewertungen."
} 


\section{Instrumentelle Umsetzung auf der Ebene des einfachen Rechts}

\section{a) Adressatentypisierung, Handlungstypisierung, Erfolgstypisierung}

Die materielle Nachverdichtung kann bei der Normativierung eines der drei offenen Parameter des Gefahrenbegriffs ansetzen: des Störers, des Rechtsguts und der Kausalbeziehung. Hierbei kann zwischen der Adressatentypisierung, der Handlungstypisierung und der Erfolgstypisierung unterschieden werden. An die Stelle des Störerbegriffs tritt z.B. der Betreiberbegriff. An die Stelle undefinierter Handlungen treten präzisierte Handlungspflichten. Schließlich lässt sich auch die Rechtsgutverletzung typisieren, etwa durch Verbotstatbestände und abstrakt-generelle Festsetzungen von Grenzwerten, ${ }^{95}$ Umweltstandards ${ }^{96}$ oder Sicherheitsstufen. ${ }^{77}$ Hier wird die Schädigung tatbestandlich antizipiert, ohne abwarten zu müssen, ob das Rechtsgut tatsächlich geschädigt wird.

\section{b) Instrumentenvielfalt}

Je genauer die Parameter normativiert werden, desto leichter lassen sich ungewisse Wahrscheinlichkeiten ermitteln und bewerten. Dabei sind solche Instrumente zur Risikoregulierung besonders geeignet, die nicht bei einer Handlungstypisierung ansetzen, weil die Vielfalt menschlicher Verhaltensmöglichkeiten eine entsprechend umfangreiche Normproduk-

\footnotetext{
${ }^{95}$ So auch W. Berg Ungewisser Sachverhalt (Fn. 3), 308-312; C. Tünnesen-Harmes Risikobewertung im Gentechnikrecht, 2000, 92f. Zum Instrument der Grenzwerte C. Paul Grenzwerte als rechtliches Mittel der Techniksteuerung, in: K. Vieweg (Hrsg.) Spektrum des Technikrechts, 2002, 21, $23 \mathrm{fff}, 29,35-37$; R. Streinz Wieviel ist Nichts? Ist Nichts zu viel?, ZLR 2002, 689, 693, 697f.; $R$. Hendler Umweltrechtliche Grenzwerte in der Gerichts- und Verwaltungspraxis, DöV 1998, 481, 481-483; W. Spannowsky Die Grenzwertkonzeption im Wandel, NVwZ 1995, 845, 846, 848f.; K. Hansmann Zur Problematik der Festsetzung von Immissionsgrenzwerten, FS Sendler (Fn. 20), 285, 290ff.; M. Wagner Definition von Grenz-, Richt- und Anhaltswerten, in: VDI Koordinierungsstelle Umwelttechnik (Hrsg.) Grenzwerte und Grenzwertfindung, 1990, 11; G. R. Baum Grenzwertfindung im Umweltschutz aus politischer Sicht, ebd., 33.

${ }_{96}$ E. Rehbinder Ein Modell für die Setzung von Umweltstandards, FS Feldhaus, 1999, 141, $143 \mathrm{ff}$; R. Steinberg Standards des integrierten Umweltschutzes, NuR 1999, 192; A. Cansier/D. Cansier Umweltstandards bei Unsicherheit aus entscheidungstheoretischer Sicht, ZAU Sonderheft 10/1999, 157; G. Lübbe-Wolf Regelbildung versus Entscheidungsspielräume für die Umweltbehörden, in: dies./E. Gawel (Hrsg.) Effizientes Umweltordnungsrecht, 2000, 151; C. Streffer u.a. Umweltstandards, 2000, $381 \mathrm{ff}$.

${ }^{97}$ Gentechnik-Sicherheitsverordnung in der Fassung v. 14. 3. 1995 (BGBI I S. 297, geändert durch Gesetz v. 16. 8. 2002, BGBl I S. 3230) aufgrund von § 7 Abs. 1 GenTG. Zur typisierenden Bewertung durch Sicherheitsstufen C. Tünnesen-Harmes (Fn. 95), 94ff., $109 \mathrm{ff}$; $M$. Marx Der Sicherheitsstandard der Betreiberpflichten im Gentechnikrecht, $1997,133,277 \mathrm{ff}$.
} 
tion erforderte. Abstrakt-generelle Erfolgstypisierungen durch Grenzwerte ${ }^{98}$ versprechen genauso Erfolg wie Adressatentypisierungen, die das konkrete Verhalten dem Adressaten überlassen. Ein Muster der Risikozurechnung durch Adressatentypisierung ist die Gefährdungshaftung, ${ }^{99}$ ein historisch ${ }^{100}$ wie ökonomisch ${ }^{101}$ bewährtes Instrument. ${ }^{102}$ Die hierdurch erreichte Individualisierung des Risikos kann über Versicherungen

${ }^{98} \mathrm{Zu}$ den Vorzügen von Grenzwertfestsetzungen auch N. Luhmann Grenzwerte der ökologischen Politik. Eine Form von Risikomanagement, in: Risiko und Regulierung (Fn. 55), 195, 202, 206ff.; R. Wolf Antiquiertheit (Fn. 50), 386f.; M. Böhm Rechtliche Probleme der Grenzwertfindung im Umweltrecht, UPR 1994, 132; G. Winter Einleitung, in: ders. (Hrsg.) Grenzwerte, 1986, 1, 23f. Anderer Ansicht K.-H. Ladeur Alternativen zum Konzept der „Grenzwerte“ im Umweltrecht - Zur Evolution des Verhältnisses von Norm und Wissen im Polizeirecht und im Umweltplanungsrecht, ebd., 263, der die Erfolgstypisierung als „Scheingewissheit" tadelt, dadurch aber den normativen Vorzug wieder zu einem vermeintlich realitätswidrigen Nachteil stilisiert.

$99 \mathrm{Zu}$ Sinn und Rechtfertigung der Gefährdungshaftung vgl. K. Larenz/C.-W. Canaris Lehrbuch des Schuldrechts, Band II/2, 13. Aufl. 1994, $\S 84 ; H$. Kötz Haftung für besondere Gefahr - Generalklausel für die Gefährdungshaftung, AcP 170 (1970), 1, $14 \mathrm{ff}$., $22 \mathrm{ff}$.; immer noch einschlägig R. Müller-Erzbach Gefährdungshaftung und Gefahrtragung, AcP 109 (1912), 1, $57 \mathrm{ff}, 142$.

100 Vgl. I. vom Feld Dampfkesselüberwachung (Fn. 4); M. Eckardt Technischer Wandel und Rechtsrevolution. Ein Beitrag zur ökonomischen Theorie der Rechtsentwicklung am Beispiel des deutschen Unfallschadensrechts im 19. Jahrhundert, 2001, $213 \mathrm{ff} ., 249 \mathrm{ff}$.; R. Ogorek Untersuchungen zur Entwicklung der Gefährdungshaftung im 19. Jahrhundert, $1975,61 \mathrm{ff} ., 98 \mathrm{ff}$.

${ }_{101}$ P. Salje Technikrecht und Ökonomische Analyse, in: Handbuch des Technikrechts (Fn. 16), 73, 90; J. Schmidt Risikoregulierung (Fn. 61), 287 ff.; P. Trimarchi Die Regelung der Vertragshaftung aus ökonomischer Sicht, ZHR 136 (1972), 118, $127 \mathrm{ff}$., der als Vorzüge der Gefährdungshaftung anführt: Gesamtwirtschaftlich effizienter Präventionsaufwand, Druckmittel zur Beschleunigung des technischen Fortschritts, Risikozurechnung auf das Unternehmen, Kostenreduzierung durch Beweislastvereinfachung. Des Weiteren: M. Adams Ökonomische Analyse der Gefährdungs- und Verschuldenshaftung, 1984, 36ff.; J. Taupitz Ökonomische Analyse und Haftungsrecht, AcP 196 (1996), 114; G. Calabresi The Cost of Accidents, 1970.

102 Gefährdungshaftungstatbestände finden sich zwar auch in Umwelt- und Technikgesetzen, als systematisches Instrument eines Risikoverwaltungsrechts ist die Gefährdungshaftung aber im öffentlichen Recht nicht hinreichend entwickelt, weil sie sich mit dem Verursacherprinzip schwer verträgt; so auch $M$. Kloepfer/T. Brandner Umweltrecht, 2. Aufl. 1998, $\S 6$ Rn. 54. Vor allem $\S 1$ UmwHG; $\$ 1$ HaftPflG; $\S 22$ WHG; $\$ 25$ ff. AtG; $\S 84$ AMG; $\$ \S 32 \mathrm{ff}$. GenTG; anwendungsreduziert auch in $\S 14 \mathrm{~S} .2 \mathrm{BImSchG}$. Dazu P. Salje Anlagenhaftungsrecht, in: Handbuch des Technikrechts (Fn. 16), 271, 284, 314; K. Vieweg Produkthaftungsrecht, ebd., 329; R. Schwarze Präventionswirkungen des Umwelthaftungsgesetzes und der Umwelthaftpflichtversicherung, in: C. Ott/H.-B. Schäfer (Hrsg.) Die Präventivwirkung zivil- und strafrechtlicher Sanktionen, 1999, 206; P.-T. Stoll Sicherheit als Aufgabe von Staat und Gesellschaft, 2003, $213 \mathrm{ff}$.; H. Werner Gentechnikhaftung, 1996. 
privatwirtschaftlich kalkuliert und verteilt werden, ${ }^{103}$ so dass das Recht mittelbar auf ökonomische Rationalität zurückgreift. Aus demselben Grund erstrebenswert ist auch die nähere normative Ausgestaltung von Sachherrschaftsstellungen. Die Zustandsverantwortlichkeit ist ein $\mathrm{Zu}-$ rechnungsmodell, das sich für normative Nachverdichtung vorzüglich eignet. ${ }^{104}$ Ziel muss es immer sein, Risiken zu individualisieren, sie also als das zu begreifen, was sie ursprünglich waren: Folgen intendierter Entscheidungen. ${ }^{105}$ Mittel dazu sind etwa Informationsrechte, die über Risiken aufklären, ${ }^{106} \mathrm{Maßnahmen,} \mathrm{die} \mathrm{individuellen} \mathrm{Fehleinschätzungen}$ durch ökonomische Anreize entgegenwirken, ${ }^{107}$ oder die rechtliche Allokation von Umweltgütern, ${ }^{108}$ etwa über Emissionszertifikate. ${ }^{109}$ Auch

103 G. Wagner Haftung und Versicherung als Instrument der Techniksteuerung, VersR 1999, 1441; J. Taupitz Umweltschutz durch zivilrechtliche Haftung, in: F. Nicklisch (Hrsg.) Umweltrisiken und Umweltprivatrecht im deutschen und europäischen Recht, 1995, 21; P. Marburger Grundsatzfragen des Haftungsrechts, AcP 192 (1992), 1. Zu den Grenzen der Ermittlung und Bewertung versicherungstechnischer Risiken (Zufallsrisiko, Irrtumsrisiko, Diagnoserisiko, Prognoserisiko): J. Schmidt Risikoregulierung (Fn. 61), $297 \mathrm{ff}$; P. Fleck Einschätzbarkeit und Versicherbarkeit von Umweltrisiken, in: F. Wiebecke (Hrsg.) Umwelthaftung und Umwelthaftungsrecht, 1990, 70.

104 Zur Leistungsfähigkeit der Zustandsverantwortlichkeit sowohl als Grundmodell als auch als noch nicht hinreichend genutztes umweltrechtliches Instrument $O$. Lepsius Besitz (Fn. 60), 220ff., 502 ff.; E. Rehbinder Ziele (Fn. 11), Rz. 102; ambivalent K.-H. Ladeur Risikooffenheit (Fn. 91), 120, 135f., der zwar die Vorzüge der Zustandsverantwortlichkeit gegenüber Kausalitätsproblemen anerkennt, ihre normative Nachverdichtung aber nicht für möglich hält.

$105 \mathrm{Vgl}$. oben I.

$106 \$ 4$ Abs. 1 UIG, $\S 3$ a, 9 UVPG; $\$ 14$ Abs. 2 UAG; $\$$ 31, 46a BImSchG; $\$ 12$ Immissionswerte-VO (22. BImSchVO); B. Wegener Transparenz und Geheimhaltung im Recht der Gentechnik, in: J. Lege (Hrsg.) Gentechnik (Fn. 3), 147, $150 \mathrm{ff}$.

107 Gesetz über die Einführung der ökologischen Steuerreform (BGB1 I 1999, 378); Gesetz über die Fortführung der ökologischen Steuerreform (BGBl I 1999, 2432; I 2000, 440); § 1 UmwHG; § 3b KraftStG; \$§ 2b-4 MineralölStG; \$§ 3 ff. Erneuerbare-EnergienGesetz; $\$ \$ 6 \mathrm{ff}$. Verpackungs-VO. Zum Instrumententyp E. Gawel Rationale Gefahrenabwehr. Marktsteuerung und ökologische Risiken, in: Effizienz im Umweltrecht (Fn. 11), 249.

108 Zur Notwendigkeit rechtlicher Allokationen für ökonomische Effizienzkalkulationen E. Gawel Umweltallokation durch Ordnungsrecht, 1994, 114ff., $148 \mathrm{ff}$. Siehe auch ders. Ökonomische Analyse des Umweltverwaltungsrechts, StWStP 4 (1993), 553; ders. Umweltordnungsrecht - ökonomisch irrational? in: Rationale Umweltpolitik (Fn. 86), 201.

109 Entwurf der EU-Kommission für eine Rahmen-Richtlinie für den Emissionshandel mit Treibhausgasen, KOM (2001), 581. Zum Stand der Richtlinie und Umsetzungsproblemen J. Schlüter Emissionsrechtehandel ante portas, NVwZ 2003, 1213; M. Burgi Die Rechtsstellung der Unternehmen im Emissionshandelssystem, NJW 2003, 2486, 2486-2488; A. Epiney Emissionshandel in der EU, DVBl 2002, 579, 579f.; A. Voßkuhle 
können Gefahrerforschungseingriffe vermieden werden, wenn Betreibern die Risikoermittlung übertragen oder Mitteilungspflichten auferlegt werden. ${ }^{110}$

Keine Allheilmittel sind zu erwarten. Vielmehr empfiehlt es sich, unterschiedliche Zurechnungen vorzunehmen, um möglichst viele Wahrscheinlichkeitsrelationen normativ zu erfassen. ${ }^{111}$

\section{c) Unzweckmäßige Relationierungen}

Nicht wenige Gesetze werden dem Anspruch an eine normative Nachverdichtung nicht gerecht. Gesetze, die auf den Stand von Wissenschaft und Technik verweisen, ${ }^{112}$ verlangen ohne normative Eingrenzung eine schon erkenntnistheoretisch nicht mit Gewissheit zu treffende technische Aussage, ${ }^{113}$ die durch den Bezug auf die Wissenschaft zusätzlich dynamisiert wird. ${ }^{114}$ Mit Wissenschaft und Technik werden unterschiedliche Er-

Rechtsfragen zur Einführung von Emissionszertifikaten, in: UTR 61, 2002, 159, $176 \mathrm{ff}$; H.-W. Rengeling (Hrsg.) Klimaschutz durch Emissionshandel, 2001; U. Hösch Zur rechtlichen Beurteilung von Emissionszertifikaten als Instrument der Vorsorge im Umweltrecht, in: UTR 58, 2001, 127; B. Stüer/H. Spreen Emissionszertifikate - Ein Plädoyer zur Einführung marktwirtschaftlicher Instrumente in die Umweltpolitik, UPR 1999, 161; H. Bonus (Hrsg.) Umweltzertifikate, 1998; C. Koenig Möglichkeiten und Grenzen von Zertifikatsmärkten als Steuerungsmedien im Umweltrecht, DÖV 1996, 943.

$110 \$ 10$ Abs. 1 Satz 2 und 3, 29 BImSchG; $\$ 8$ Abs. 1, 21 GenTG; $\$ \$ 6,7,12 \mathrm{i}$ ChemG; $\$ \S 22,25,29,63 \mathrm{a}-68$ AMG.

111 Überblicke über die Vielfalt gesetzlicher Regelungsansätze bei unterschiedlichen Regelungsgegenständen: P.-T. Stoll Sicherheit (Fn. 102), zusammenfassend 262f.; H.-H. Trute Staatliches Risikomanagement (Fn. 15), $64 \mathrm{ff}$., $85 \mathrm{ff}$.; G. Lübbe-Wolff Modernisierung (Fn. 38), 102 ff. zur Geeignetheit zahlreicher Instrumente unter dem Aspekt der Zurechenbarkeit und Vollziehbarkeit. Am Beispiel des Umweltrechts L. Krämer Direkte und indirekte Verhaltenssteuerung, in: H.-W. Rengeling (Fn. 26), $\S 15 \mathrm{Rn} .14 \mathrm{ff}$., $48 \mathrm{ff}$.; E. Rehbinder Ziele (Fn. 11), Rn. $129 \mathrm{ff}$, $179 \mathrm{ff}$.; P. Koller Die rechtliche Umsetzung ökologischer Forderungen, in: Ökologische Ethik und Rechtstheorie (Fn. 51) 127, 147; aus ökonomischer Sicht: E. Gawel Umweltpolitik durch gemischten Instrumenteneinsatz. Allokative Effekte instrumentell diversifizierter Lenkungsstrategien für Umweltgüter, 1991. Am Beispiel des Chemikaliengesetzes aus soziologischer Sicht $R$. Münch Risikopolitik, 1996, $73 \mathrm{ff} ., 180 \mathrm{ff}$.

112 „Stand von Wissenschaft und Technik“ z.B.: $\$ 7$ Abs. 2 Nr. 3 AtG; $\$ \$ 6$ Abs. 1b, 7 Abs. 2, 11 Abs. 1 Nr. 4, 16 Abs. 1 Nr. 2 GenTG; "Stand der Technik“ z. B.: $\$ \$ 5$ Abs. 1 Nr. 2, 14, 22 Abs. 1 Nr. 1 und 2, 41 Abs. 1, 48 Nr. 2, 52 Abs. 1 Nr. 2 BImSchG; $\S 19$ Abs. 3 ChemG; „Stand der Wissenschaft“ z. B.: $\$ \$ 7$ Abs. 1 Nr. 1-4 GenTG.

113 Vgl. D. Lohse Der Rechtsbegriff „Stand der Wissenschaft" aus erkenntnistheoretischer Sicht, 1994, $128 \mathrm{ff}$., mit dem Ergebnis, dass die Gefahrenabwehr nicht durch das menschliche Erkenntnisvermögen begrenzt wird, sondern durch normative Festlegung.

114 Dazu R. Steinberg Verfassungsstaat (Fn. 11), 105-110; R. Wolf Technik (Fn. 4), $257 \mathrm{ff}$. Im Einzelfall ist Dynamisierung kein Problem; erst ihre Typisierung führt zu einem inneren Widerspruch, denn eine typisierte Dynamik ist nicht mehr dynamisch. Mit den Mit- 
kenntnisgrößen verknüpft, die in einem Zielkonflikt zueinander stehen. ${ }^{115}$ Hier wird ein nicht relationierbares Wahrscheinlichkeitsurteil erwartet, das die Trennung von Möglichkeit und Wahrscheinlichkeit verwischt und zu einer Ermächtigung technokratischen Sachverstandes führt.116 Rechtsstaatlich ist dieses Vorgehen genauso bedenklich wie vollzugstechnisch problematisch. ${ }^{117}$

Auch finale Normprogramme ${ }^{118}$ verursachen erhebliche Rechtsprobleme, ${ }^{119}$ weil sie das Nebeneinander unterschiedlicher Erwartungen (technischer, ökonomischer, ethischer) nicht in juristische Zurechnungen umsetzen, sondern als politische Aufgabe anderen Entscheidungsträgern überlassen, die institutionell dafür nicht ausgewiesen sind. ${ }^{120}$

teln der abstrakt-generellen Rechtsetzung kann daher Dynamik nicht erzielt werden. Nur die Normen selbst können in einem politischen Sinne durch Normänderung dynamisiert werden.

115 Vgl. K.-H. Ladeur Umweltrecht (Fn. 2), 89: Die Wissenschaft entwickelt Hypothesen und wirft neue Fragen auf; die Technik soll sie begrenzen und die Verfügbarkeit und Praktikabilität von Regelwissen in einem begrenzten Zeithorizont gewährleisten; ähnlich H.-H. Trute Staatliches Risikomanagement (Fn. 15), 102; ders. Die Forschung zwischen grundrechtlicher Freiheit und staatlicher Institutionalisierung, 1994, 80-85.

116 Zur herrschaftslegitimierenden Funktion von Technik J. Habermas Technik und Wissenschaft als „Ideologie“, 1968.

117 Daher hätte BVerfGE 49, 89, 133-140, den entsprechenden Tatbestand im Atomgesetz wegen Unbestimmtheit für verfassungswidrig erklären müssen.

$118 \mathrm{Zu}$ Entwicklung und Kritik R. Breuer Konditionale und finale Rechtsetzung, AöR 127 (2002), 523, 525-527, 556ff., 566ff.; ders. Zur Lage der deutschen Verwaltungsrechtswissenschaft, Die Verwaltung 36 (2003), 271, $278 \mathrm{ff}$.; G. Lübbe-Wolf Modernisierung (Fn. 38), 146-150; C. Waldhoff Der Verwaltungszwang, D II 1 b) bb); begriffsprägend N. Luhmann Zweckbegriff und Systemrationalität, 2. Aufl. 1977, $101 \mathrm{ff}$.; dazu W. Schmidt Die Programmierung von Verwaltungsentscheidungen, AöR 96 (1971), 321.

$119 \mathrm{Vgl}$. E. Rehbinder Festlegung von Umweltzielen - Begründung, Begrenzung, instrumentelle Umsetzung, NuR 1997, 313; ders. Ziele, Grundsätze, Strategien und Instrumente, in: J. Salzwedel (Fn. 11), Teil 04.

${ }_{120}$ Dies ist etwa ein Hauptproblem amerikanischer Umweltgesetze. Gegenüber ihren unklaren Zielformulierungen behilft man sich mit ökonomischen Effizienzzielen auf der Basis eines Kosten-Nutzen-Denkens, das verspricht, die unterschiedlichen Aspekte über monetäre Vergleichbarkeit in eine normative Ordnung zu bringen. Für solche Ansätze bei der Risikoregulierung vgl. C. Sunstein/R. Pildes Reinventing the Regulatory State, University of Chicago Law Review 62 (1995), 1, 55; C. Sunstein Risk and Reason, 2002, zusammenfassend 289-295; ders. The Cost-Benefit State. The Future of Regulatory Protection, 2002, $13 \mathrm{ff}$., $33 \mathrm{ff}$., $115 \mathrm{ff}$. Sunstein vermittelt Marktinstrumente mit der Expertise aufgeklärter Wissenschaftler und Techniker. Siehe auch ders. Which Risks First? The University of Chicago Legal Forum 1997, 101. Sunstein kritisierend und stärker auf politische Zielformulierungen vertrauend $D$. Farber Eco-pragmatism. Making sensible Environmental Decisions in an Uncertain World, 1999, $35 \mathrm{ff}$., $93 \mathrm{ff}$., $165 \mathrm{ff}$. $\mathrm{Zu}$ den historischen 
Bedenklich erscheint schließlich eine Ausrichtung der Zurechnung am Verursacherprinzip, ${ }^{121}$ denn dies verlangt nach Handlungszurechnungen und privilegiert damit die regelungstechnisch schwierigste Relationierung auf Kosten einfacherer Alternativen. ${ }^{122}$ Gerade weil Verursachungszusammenhänge komplex sind, ${ }^{123}$ sollte eher auf Adressaten- oder Erfolgstypisierungen vertraut werden, die dem Leitbild des Verursacherprinzips widersprechen.

\section{Institutionelle Umsetzung auf der Ebene des Verfassungsrechts}

Die Individualisierung der Zurechnung führt notgedrungen zu mehr Rechtsetzung. Wenn man es nicht bei der offenen Relationierung des Gefahrenbegriffs belassen will, kann man ihr nicht entgehen. ${ }^{124}$ Dies wirft eine letzte Frage auf: Welche Institutionen sind geeignet, die $\mathrm{Zu}$ rechnung von Risiken zu re-individualisieren und auf diese Weise auch die sozialen Ursachen der Problemwahrnehmung zu beheben, nämlich die naturalisierte oder vergesellschaftete Zuschreibung von Risiken zu verändern?

und methodischen Problemen amerikanischer Verwaltungsgesetzgebung $O$. Lepsius Common Law (Fn. 70 ), $37 \mathrm{ff} ., 128 \mathrm{ff} ., 207 \mathrm{ff}$.

${ }^{121}$ Die frühere Kritik des Verursacherprinzips im Umweltrecht ist in den letzten Jahren einer unkritischen Kanonisierung gewichen. Kritisch noch E. Rehbinder Politische und rechtliche Probleme des Verursacherprinzips, 1973, 29ff.; M. Kloepfer Umweltschutz durch Abgaben, DÖV 1975, 593, 594; J. Schmid Verursacherprinzip und Sozialkosten, AcP 175 (1975), 222; SRU Umweltgutachten 1978, Tz. 1703; M. Adams Das „Verursacherprinzip“ als Leerformel, JZ 1989, 787; M. Grüter Umweltrecht und Kooperationsprinzip, 1990, $24 \mathrm{ff}$.; neuere Kritik bei O. Lepsius Besitz (Fn. 60), 459 ff.; A. Suchanek Normative Umweltökonomik, 2000, 48f., 83-92, 138.

122 Gegen das Verursacherprinzip auch $R$. Wolf Antiquiertheit (Fn. 50), 388f.: Die Berufung auf das Verursacherprinzip könne eher als Exkulpationsvehikel für Umweltsünder denn als verwaltungspraktisches Mittel gegen Umweltbelastungen eingesetzt werden; ähnlich U. Beck Gegengifte, 1988, 11, 218. Zu Haftungsverlagerungen und Haftungslücken, die durch die Fixierung auf das Verursacherprinzip entstehen: $O$. Lepsius Besitz (Fn. 60), 463-466.

${ }^{123}$ Wie dieses Problem mit juristischen Kausalitätstheorien bewältigt werden kann, untersucht grundlegend L. Röckrath Kausalität, Wahrscheinlichkeit und Haftung, 2004, $\$ 1$ I: Kausalität ist eine Gesetzmäßigkeit besonderer Art, die vom Gesetz und von der Rechtswissenschaft eigenständig und normativ zu entscheiden ist.

${ }^{124}$ So auch schon B. Schlink Die Bewältigung der wissenschaftlichen und technischen Entwicklungen durch das Verwaltungsrecht, VVDStRL 48 (1990), 235, $259 \mathrm{f}$. 


\section{a) Umsetzung als juristische Rationalität individuell-konkreter Rechtsetzung}

Momentan wird Risikoregulierung institutionell in erster Linie als Auftrag der Verwaltung begriffen. ${ }^{125}$ Wenn die Lösung aber nur im Wege der abstrakt-generellen und nicht der individuell-konkreten Normsetzung zu finden ist, wird Risiko als Rechtsproblem an der falschen Stelle institutionalisiert. Die Problemlösung hat eine institutionelle Komponente. Die Verwaltung müsste zur Re-Individualisierung der Zurechnung Leistungen erbringen, die sie nicht erbringen kann, denn sie müsste abstrakt-generelle Normen setzen. Sie müsste im Rahmen einer juristischen Rationalität politische Aspekte verarbeiten. Hier liegt der Grund, warum die Verwaltung mit Risikoentscheidungen oft überfordert ist. Und hier liegt auch der Grund, warum das soziale Problem des Risikos, nämlich die Vergesellschaftung und Naturalisierung der Zurechnung, nicht behoben wird, das Rechtssystem also das soziale und kognitive Risikoproblem nicht löst.

Einerseits wird das Problem der Vergesellschaftung institutionell falsch verortet, wenn man es durch die Einbeziehung partizipativer gesellschaftlicher Teilhabe bei der individuell-konkreten Rechtsetzung lösen will126 und dann als prozedurales Rechtsdenken positiv wendet. ${ }^{127}$ Hier wird ju-

${ }^{125}$ Vgl. etwa U. Di Fabio Entscheidungsprobleme der Risikoverwaltung, NuR 1991, 353; ders. Risikoentscheidungen (Fn. 2), 452: „Risikoentscheidungen als kooperativer Gestaltungsauftrag für Verwaltung und inkorporierten Sachverstand“. Zur Vielfalt aktueller „Risikoverfahren“ $A$. Voßkuhle Strukturen und Bauformen ausgewählter neuer Verfahren, in: W. Hoffmann-Riem/E. Schmidt-ABmann (Hrsg.) Verwaltungsverfahren und Verwaltungsverfahrensgesetz (Fn. 62), 277, 330-343. Am Beispiel der Normenflut im Gentechnikrecht $R$. Hasse Nicht-intendierte Effekte kooperativer Risikoregulierung bei der Implementation des Gentechnikgesetzes, in: Risiko und Regulierung (Fn. 55), 70, $74 \mathrm{ff}$., $81 \mathrm{ff} . ;$ M. Schröder Gentechnikrecht in der Praxis. Eine empirische Studie zu den Grenzen der Normierbarkeit, 2001, $183 \mathrm{ff}$., $237 \mathrm{ff}$.: Die fortschreitende Verrechtlichung zeige keine negativen Folgen.

${ }^{126}$ Zum Problem und seinen Erscheinungsformen M. Schmidt-Preuß, U. Di Fabio Verwaltung und Verwaltungsrecht zwischen gesellschaftlicher Selbstregulierung und staatlicher Steuerung, VVDStRL 56 (1997), 160, 235; I. Lamb Kooperative Gesetzeskonkretisierung, 1995, $182 \mathrm{ff} ., 195 \mathrm{ff} .232 \mathrm{ff}$.

127 K. Eder Prozedurales Recht und Prozeduralisierung des Rechts, in: Wachsende Staatsaufgaben (Fn. 60), 155, 155 ff., 161 ff.; R. Pitschas (Fn. 79), 80-87; K.-H. Ladeur Gefahrenabwehr (Fn. 25); ders. Postmoderne Rechtstheorie. Selbstreferenz - Selbstorganisation - Prozeduralisierung, 2. Aufl. 1995, 205-213; ders. Das Umweltrecht der Wissensgesellschaft, 1995; ders. Risikobewältigung durch Flexibilisierung und Prozeduralisierung des Rechts, in: Rechtliches Risikomanagement (Fn. 63), 41; R. Wolf Der ökologische Rechtsstaat als prozedurales Programm, in: A. Roßnagel/U. Neuser (Hrsg.) Reformperspektiven im Umweltrecht, 1996, 57; E. Hagenah Prozeduraler Umweltschutz, 1996, 58ff.; G.-P. Calliess Prozedurales Recht, 1999, 83 ff.; A. Scherzberg Wissen (Fn. 62), $129-131$. 
ristische mit politischer Rationalität vermischt, so dass die Komplexität der Entscheidung erhöht und nicht reduziert wird. Andererseits wird die Auflösung der Naturalisierung durch die Delegation an Experten institutionell erschwert. Daher sollten Tendenzen, die Verordnungsgebung zu verrechtlichen ${ }^{128}$ oder zur Anhörung von Expertenkommissionen zu verpflichten ${ }^{129}$ überdacht werden. Sonst liefe man Gefahr, die Naturalisierung der Zurechnung durch Sachverstand und die Vergesellschaftung durch Binnenpluralismus und Verfahrensregelungen zu bewältigen, ${ }^{130} \mathrm{mit}$ dem Effekt, dass die Vorzüge politischer Rationalität zunichte gemacht werden und die Politik aus ihrer demokratischen Verantwortung entlassen wird. ${ }^{131}$ Wie jedermann sind auch Experten zu Wahrscheinlichkeitsaus-

\footnotetext{
${ }^{128}$ Weithin wird eine partielle Kompensation von verfassungsrechtlichen Defiziten und gesetzlicher Regelungsdichte durch partizipative Elemente, sei es der Öffentlichkeitsbeteiligung, sei es der Einbindung pluralistisch besetzter Kommissionen, für möglich und erforderlich gehalten; vgl. E. Denninger Verfassungsrechtliche Anforderungen an die Normsetzung im Umwelt- und Technikrecht, 1990, 169-175; G. Lübbe-Wolff Verfassungsrechtliche Fragen der Normsetzung und Normkonkretisierung im Umweltrecht, ZG 1991, 219, 232 ff., 243f.; E. Schmidt-Aßmann Ordnungsidee (Fn. 45), 275; Bundesministerium für Umwelt, Naturschutz und Reaktorsicherheit (Hrsg.) Umweltgesetzbuch (UGB-KomE), 1998, \$§ 11-33 mit Erläuterungen 463-500; E. Rehbinder Setzung von Umweltstandards (Fn. 96), 141, 152; siehe auch C. Gößwein Allgemeines Verwaltungs(verfahrens)recht der administrativen Normsetzung, 2001. Skeptisch indes M. Böhm Der Normmensch, 1996, $15 \mathrm{ff}$.

${ }^{129}$ Z.B. die Zentrale Kommission für die Biologische Sicherheit als Sachverständigenkommission beim Robert-Koch-Institut, $\$ \S 4,5,7,11,16,30$ GenTG. Zur kooperativen Einbindung der ZKBS in die allgemein-abstrakten und konkret-individuellen Entscheidungsprozesse W. Graf Vitzthum/T. Geddert-Steinacher Standortgefährdung, 1992, 107-118. Zur Letztverantwortung der Behörde trotz praktischer Entscheidungshoheit der ZKBS R. Wahl (Fn. 15) 24. Lfg. 1996, $\$ \$ 4,5$ GenTG Rn. 19f. (die Mitwirkungsbefugnisse gehen faktisch über den Bereich rechtlich unerheblicher Verfahrenshandlungen hinaus); G. Hirsch/A. Schmidl-Didczuhn GenTG (Fn. 30), § 1 Rn. 61. Kritisch zur Einbindung der ZKBS in den Normierungsprozess schon D. Murswiek Bewältigung (Fn. 16), 220f.

130 Skeptisch zur Rolle beratender Kommissionen als Form der institutionalisierten Öffentlichkeitsbeteiligung SRU Umweltgutachten 2002, Tz. $146 \mathrm{f}$.

${ }_{131} \mathrm{Vgl}$. A. Bora Differenzierung und Inklusion. Partizipative Öffentlichkeit im Rechtssystem moderner Gesellschaften, 1999, $183 \mathrm{ff}$. zu konkurrierenden Rationalitäten und Erwartungen bei der Öffentlichkeitsbeteiligung. Generell die unzureichende demokratische Legitimation und Kontrolle des deutschen Umweltrechts und die zu große Eigenverantwortlichkeit der vollziehenden Gewalt kritisierend $S$. Rose-Ackerman Umweltrecht (Fn. 50), insbes. 14-31, 133-137 (harte Kritik am untergesetzlichen Regelungswerk); vgl. als Grundkritik etwa 182: „Die überkommene Auffassung in der Bundesrepublik zur unpolitischen und rein fachlichen Natur höherstufiger politisch-programmatischer Gestaltungsprozesse der Exekutive erschwert deren Reform. Die Rechtslehre in der Bundesrepublik sollte die inneradministrativen Verfahren daher einer kritischen Würdigung unterziehen, ...“.
} 
sagen nur in der Lage, wenn eine normative Relationierung vorgegeben ist. ${ }^{132}$ Ohne normativ vorgegebene Fixpunkte müssten Sachverständige eine ihrer Natur nach politische Aufgabe lösen. ${ }^{133}$ Weil Risikozurechnung eine abstrakt-generelle Normsetzung erfordert, kann ihre Rolle nur eine beratende, keine entscheidende sein. Daher sollte eine Beratungskultur institutionalisiert werden, ${ }^{134}$ die den politischen Charakter der Normsetzung erhält und ihn nicht naturalisiert. ${ }^{135}$

\section{b) Umsetzung als politische Rationalität abstrakt-genereller Rechtsetzung}

Risiko als Rechtsproblem kann nur mit abstrakt-genereller Rechtsetzung bewältigt werden. Daher handelt es sich um ein politisches Problem,

${ }^{132} \mathrm{Vgl}$. BVerfGE 83, 130, 140f.: In einer wissenschaftlich ungeklärten Situation sei der Gesetzgeber befugt, die Gefahrenlagen und Risiken abzuschätzen und zu entscheiden, ob er Maßnahmen ergreifen will oder nicht. Was das Bundesverfassungsgericht hier für psychische Schäden von Kindern und Jugendlichen durch Pornographie ausgesprochen hat, gilt auch für technische Gefahren, zumal das Gericht die Kalkar-Entscheidung heranzieht, ebd., $141 \mathrm{f}$. Andernfalls würden unterschiedliche Maßstäbe angelegt, die nur durch den Glauben an eine naturwissenschaftliche Objektivität gerechtfertigt werden könnten, in der Annahme, diese sei einer psychischen Subjektivität überlegen.

133 Wenn von der Wissenschaft nicht erfüllbare relationslose Wahrscheinlichkeitsurteile erwartet werden, droht ein Verlust ihrer Glaubwürdigkeit, der erst recht Krisengefühle auslösen wird. Vgl. H. Theisen Zur Demokratieverträglichkeit von Bio- und Gentechnologie, Soziale Welt 42 (1991), 109, 118-120; R. Münch Risikopolitik, 1996, 190-192; C. Lau (Fn. 80), 258-262; U. Beck/S. May Gewußtes Nichtwissen und seine rechtlichen und politischen Folgen, in: Modernisierung der Moderne (Fn. 48), 247, 250, 255; ironische Beschreibung bei $W$. Lübbe Epistemische Pflichten in der „Wissensgesellschaft", in: Wissen (Fn. 62), 145 ff., 381 f. Für eine politische Funktion einer nur wissenschaftlicher Rationalität verpflichteten neutralen Beamtenschaft (vor dem - anders gelagert - USamerikanischen Problemhintergrund) hingegen S. Breyer Breaking the Vicious Circle. Toward Effective Risk Regulation, 1993, 59-68, 80f.

134 In jüngster Zeit wird ein Funktionswandel des Expertenwissens beobachtet, der einen dissonanten Expertendiskurs als unabdingbare Voraussetzung für die erfolgreiche Behauptung einer politischen Handlungsfähigkeit begreift, vgl. A. Bogner/W. Menz Wissenschaftliche Politikberatung? Der Dissens der Experten und die Autorität der Politik, Leviathan 30 (2002), 384, 397; vgl. auch schon H. Hartmann/M. Hartmann Vom Elend der Experten, Kölner Zeitschrift für Soziologie und Sozialpsychologie 34 (1982), 193.

135 Etwa durch die Institutionalisierung der (beratenden) Risikobewertung oder Technikfolgenabschätzung. Vgl. A. Grunwald Technikfolgenabschätzung. Konzeptionen und Kritik, in: ders. (Hrsg.) Rationale Technikfolgenbeurteilung, 1999, 11; ders. Technikeinführung als gesellschaftlicher Lernprozeß. Zur Rolle von Politik und Technikfolgenabschätzung, in: Technikumsteuerung (Fn. 23), 43, 52ff.; T. Petermann/R. Coenen (Hrsg.) Technikfolgenabschätzung in Deutschland, 1999; vgl. auch Bericht der Enquete-Kommission des Deutschen Bundestages „Einschätzung und Bewertung von Technikfolgen“ BT-Drs. 10/5844. Allgemein M. Schwab Rechtsfragen der Politikberatung im Spannungsfeld zwischen Wissenschaftsfreiheit und Unternehmensschutz, 1999. 
das die kognitiven Fähigkeiten des Parlaments erfordert. ${ }^{136}$ Der politische Prozess vermag die Voraussetzungen für eine normative Relationierung zu erbringen, ${ }^{137}$ nämlich Informationen zu gewinnen, schnell zu handeln, Fehlentwicklungen zu korrigieren und strenger Kontrolle durch die politische Öffentlichkeit zu unterliegen. ${ }^{138}$ Aber auch weil Risiken subjektiv unterschiedlich wahrgenommen werden, wie uns die psychologische Forschung lehrt, ${ }^{139}$ muss über ihre Bewertung ein intersubjektiver Konsens hergestellt werden. ${ }^{140}$ Die richtige Rechtsform gesellschaftlicher Lernfähigkeit ist daher die abstrakt-generelle Normsetzung. ${ }^{141}$

${ }^{136}$ Denn dies ist der institutionelle Ort, an dem die begrenzte Erkenntnisfähigkeit des Subjekts, die gerade die Ursache für die kollektive Zuschreibung von Risiken ist, durch abstrakt-generelle Rechtsetzung behoben wird. Die abstrakt-generelle Rechtsetzung bedarf dann freilich wieder einer Umsetzung durch den Normanwender im Sinne einer individuell-konkreten Rechtsetzung, die eine eigene, subjektive Erkenntnisleistung ist. Dazu näher $O$. Lepsius Die erkenntnistheoretische Notwendigkeit des Parlamentarismus, in: M. Bertschi u.a. (Hrsg.) Demokratie und Freiheit, 1999, 123, 146-164.

${ }^{137}$ BVerfGE 49, 89, $130 \mathrm{ff}$;; 56, 54, 78f.; A. Roßnagel Die parlamentarische Verantwortung für den technischen Fortschritt, ZRP 1992, 55; P.-T. Stoll (Fn. 102), 432-447, 459: Das Thema Sicherheit und Risikobewältigung habe sich erst langsam an das Parlament herangearbeitet. D. Farber Eco-pragmatism (Fn. 120), $5 \mathrm{ff}$., $51 \mathrm{ff}$., 115; Enquete-Kommission „Gestaltung der technischen Entwicklung“ BT-Drs. 11/4606.

138 Vgl. K. v. Beyme Der Gesetzgeber. Der Bundestag als Entscheidungszentrum, 1997, mit umfangreichen empirischen Belegen; R. Zintl Politisches Wissen und Wissen in der Politik, in: Wissen (Fn. 62), 93, $100 \mathrm{ff}$.; ferner H. Schulze-Fielitz Theorie und Praxis der parlamentarischen Gesetzgebung, 1988.

139 Dazu Bayerische Rück (Hrsg.) Risiko ist ein Konstrukt. Wahrnehmungen zur Risikowahrnehmung, 1993; vor allem aber die Forschungen von P. Slovic (Hrsg.) The Perception of Risk, 2000, darin u.a.: B. Fischhoff u.a. How Safe is Safe Enough?, 80; P. Slovic u.a. Facts and Fears: understanding Perceived Risk, ebd., 137; P. Slovic Perception of Risk, ebd., 220, zuerst in: Science 236 (1987), 280; P. Slovic Informing and Educating the Public about Risk, ebd., 182; zur demokratischen Aufklärung und Artikulation von Risiken ders. Perceived Risk, Trust and Democracy, ebd., 316; H. Jungermann/ ders. Die Psychologie der Kognition und Evaluation von Risiko, in: Risiko und Gesellschaft (Fn. 7), 167; D. Kahneman/A. Tversky Judgement Under Uncertainty: Heuristics and Biases, 1990, 3-20.

${ }^{140} \mathrm{Zur}$ rechtlichen Bedeutung von divergierenden Risikowahrnehmungen $C$. Engel Rechtliche Entscheidungen unter Unsicherheit, in: Wissen (Fn. 62), 305, 324ff.; C. Sunstein Risk and Reason (Fn. 120), $28 \mathrm{ff}$., $53 \mathrm{ff}$.; C. Sunstein/R. Pildes Regulatory State (Fn. 120), 55-64; C. Sunstein Incommensurability and Valuation in Law, Michigan Law Review 92 (1994), 779; A. Wildavsky Citizenship in Science, in: ders. But is it true? (Fn. 55), 395. Vgl. auch J. Dewey Freedom (Fn. 58), 102ff., 113f.: Differenzen in der Technikbewertung sind ein sozio-kulturelles Problem. "The answer given to this challenge is bound up with the fate of democracy."

141 Der Gedanke wird in Rechtsprechung und Literatur oft als Pflicht des Gesetzgebers behandelt, die Angemessenheit der Regelungen laufend zu überprüfen, anzupassen und nachzubessern. Er sollte indes vor allem auf den Verordnungsgeber gemünzt sein. Zur 
Mit der Rechtsverordnung kann sie flexibel und dynamisch ${ }^{142}$ auf technische und soziale Veränderungen reagieren. ${ }^{143}$ Neben dem Parlamentsgesetz ist auch die Verordnungsgebung als eine Handlungsform des politischen Prozesses zu verstehen. Daher sollte die exekutive Normsetzung - entgegen einem vorherrschenden Trend ${ }^{144}$ - nicht zu stark verrechtlicht werden, sonst könnte sie ihre Aufgabe zur flexiblen und schnellen Normierung nicht mehr wahrnehmen und entwickelte sich zu einer schwerfälligen Ersatzgesetzgebung. ${ }^{145}$

Nicht nur der Gesetzesvorbehalt, sondern auch die Delegationsschranken und der Bestimmtheitsgrundsatz bedürfen daher im Hinblick auf die jeweiligen Erkenntnisleistungen der Institutionen stärkerer Konturierung. ${ }^{146}$ Hier kann sich aus der Risikoregulierung ein Argument zur Abgrenzung zwischen gesetzlicher und untergesetzlicher Regelung ergeben:

Nachbesserungspflicht des Gesetzgebers: BVerfGE 49, 89, 130, 132; 50, 290, 335f.; 56, 54, 82 ff.; 88, 203, 310f.; vgl. F. Ossenbühl Not des Gesetzgebers (Fn. 16), 28f.; C. Mayer Die Nachbesserungspflicht des Gesetzgebers, 1996; H. Schulze-Fielitz Zeitoffene Gesetzgebung (Fn. 8), 162-166; R. Steinberg Verfassungsgerichtliche Kontrolle der „Nachbesserungspflicht“ des Gesetzgebers, Der Staat 26 (1987), 161; P. Badura Die verfassungsrechtliche Pflicht des gesetzgebenden Parlaments zur „Nachbesserung“ von Gesetzen, FS Eichenberger, 1982, 481, 484ff. Vgl. auch K.-H. Ladeur Risikobewertung (Fn. 86), 124: "gesetzlich zu strukturierender planerischer Experimentierspielraum“.

${ }_{142}$ J. Ipsen Bewältigung (Fn. 11), 188f.; F. Nicklisch Umgang mit dem Ungewissen (Fn. 57), 2290. Zur Bedeutung von Dynamik und Wandel für die Rechtsetzung auch D. Farber Eco-pragmatism (Fn. 120), 163-190.

143 Allseits wird daher die unverzichtbare Flexibilität der Rechtsverordnungen betont: M. Kloepfer Instrumente des Technikrechts, in: Handbuch des Technikrechts (Fn. 16), 111, 137; J. Ipsen Bewältigung (Fn. 11), 189, 191; ders. Diskussionsbemerkung, VVDStRL 48 (1990), 300; H. Schulze-Fielitz Technik und Umweltrecht (Fn. 16), 453, 457f.; F. Ossenbühl Die Bewertung technischer Risiken bei der Rechtsetzung, DÖV 1982, 833, 840.

144 Vgl. Fn. 128.

145 Diese Entwicklung wird sowohl durch verfassungsrechtliche Zustimmungserfordernisse (Art. 80 Abs. 2 GG) als auch durch einfachrechtliche Formen des Zustimmungsvorbehalts des Bundestages verstärkt, z. B. $\$ 3$ Abs. 1 UVPG; $\S 51$ Abs. 2 EStG. Neuerdings unterwirft der Gesetzgeber die Verordnung sogar Änderungsvorbehalten des Bundestages, der damit die Verordnungsgebung inhaltlich an sich ziehen kann und die Form des Gesetzes durch die Form der Rechtsverordnung austauscht, z.B. $\$ 59 \mathrm{KrW}-/ \mathrm{AbfG} ; \S 48 \mathrm{~b}$ BImSchG; $§ 20$ Abs. 2 UmwHG. Vgl. zu den verfassungsrechtlichen Problemen dieser Beteiligungsform J. Schmidt Die Beteiligung des Bundestages beim Erlaß von Rechtsverordnungen, 2002, $96 \mathrm{ff}$; C. Pegatzky Parlament und Verordnungsgeber, 1999, $147 \mathrm{ff}$; A. Uhle Parlament und Rechtsverordnung, 1999, $463 \mathrm{ff}$; J. Saurer Die Mitwirkung des Bundestages an der Verordnungsgebung nach $\S 48 b$ BImSchG, NVwZ 2003, 1176.

146 Die soziale Leistung des Rechts, die kognitiven Risikozuschreibungen durch juristische Zurechnungen aufzulösen, muss auch institutionell erbracht werden. Die Lösung kann daher nicht in einer partizipativen oder expertokratischen Nachverdichtung der vollziehenden Gewalt liegen, sondern in der Vergewisserung des Gesetzesvorbehalts. 
Die Auflösung der vergesellschafteten Zuschreibung durch eine juristische Zurechnung ist eine parlamentarische Aufgabe gesetzlicher Zielformulierung, während die Auflösung der naturalistischen Zuschreibung über Grenzwerte mit Rechtsverordnungen zu bewältigen ist.

Diese Abstufungen bei der Normsetzung sollten auch bei der zukünftigen Ausgestaltung des Systems der Risikoanalyse ${ }^{147}$ zugrundegelegt werden. ${ }^{148} \mathrm{Im}$ System $^{149}$ von Risiko-Bewertung, Risiko-Management und Risiko-Information sind die normativen Elemente durch den (delegierenden) Gesetzgeber zu erbringen, die Vollzugselemente durch die Exekutive. ${ }^{150}$

Die Aufgabe der Risikozurechung wirft daher auch die Frage auf, ob die notwendige normative Nachverdichtung nicht durch die Konstitutio-

147 Zur Risikoanalyse schon D. Murswiek Bewältigung (Fn. 16), 208-212; I. Appel Europas Sorge (Fn. 39), 396f;; S. Werner Vorsorgeprinzip (Fn. 39), 338f. Neuere Fallstudien zur Umsetzung im Gentechnikrecht: $S$. Pohl Die Risikobewertung bei der Freisetzung gentechnisch veränderter Organismen, 2002, $118 \mathrm{ff}$., $171 \mathrm{ff}$; im Lebensmittelrecht $J$. Gelbert Die Risikobewältigung im Lebensmittelrecht, 2001, 56ff., 248ff.; A. Meier (Fn. 25); S. Schlacke Risikoentscheidungen im europäischen Lebensmittelrecht, 1998; im Chemikalienrecht G. Winter (Hrsg.) Risk Assessment and Risk Management of Toxic Chemicals in the European Community, 2000; am Beispiel des Strahlenrisikos Akademie der Wissenschaften zu Berlin Umweltstandards, 1992.

148 Die Risikoanalyse orientiert sich momentan noch zu sehr am naturwissenschaftlichen Möglichkeitsdenken. Sie darf nicht den Grundfehler machen, relationslose Wahrscheinlichkeitsurteile zu erwarten. Daher sollte sie nicht als Abstufung eines rein wissenschaftlichen Erkenntnisprozesses verstanden und als solcher innerhalb der Verwaltung organisiert werden, sondern analog der verfassungsrechtlichen Trennung von generellabstrakter Normsetzung und individuell-konkreter Normanwendung ausgestaltet werden.

149 Vgl. schon Grünbuch über die allgemeinen Grundsätze der Lebensmittelsicherheit, KOM (97) 176 endg. v. 30. 4. 1997; Mitteilung „Gesundheit der Verbraucher und Lebensmittelsicherheit“, KOM (97) 183 endg. v. 30.4. 1997; sodann Mitteilung der Kommission zur Anwendbarkeit des Vorsorgeprinzips (Fn. 39), Punkte 5, 5.1., 6.2.; Art. 6 Lebensmittel-Basisverordnung (178/2002/EG); $\$ \S 1,2$ Gesetz über die Errichtung eines Bundesinstituts für Risikobewertung (BfRG) v. 6. 8. 2002, BGBl. I, 3082.

150 Ähnlich $R$. Breuer Probabilistische Risikoanalysen und Gentechnikrecht, NuR $1994,157,160$, der die Risikobewertung nicht naturwissenschaftlich, sondern normativ versteht. Zur Risikobewertung als Verschränkung einer individuellen, gesellschaftlichen und staatlichen Bewertung Akademie der Wissenschaften zu Berlin (Fn. 147), $227 \mathrm{ff}$. Siehe auch C. Tünnesen-Harmes (Fn. 95), 54ff., 60, 90f. zur Notwendigkeit normativer Wertentscheidungen im Rahmen der Risikobewertung; A. Karthaus (Fn. 16), 140-144. Zur ordnungsrechtlichen Ausgestaltung der Risikoanalyse R. Steinberg Staatliches Risikomanagement im europäischen Anlagenrecht, in: E. Riedel (Hrsg.) Risikomanagement (Fn. 15), 17, 21 ff.; C. Koenig Internalisierung des Risikomanagements durch neues Umwelt- und Technikrecht?, NVwZ 1994, 937, 937-939. 
nalisierung gesetzlicher Inhalte ${ }^{151}$ oder die Verrechtlichung politischer Handlungsformen instrumentell behindert und institutionell falsch zugeordnet wird. Womöglich verrechtlichen wir am falschen Platz, wohl auch, weil wir der Rationalität politischer Institutionen zu wenig zutrauen und das Rechtsstaatsprinzip über das Demokratieprinzip stellen.

\section{Der Mensch als Risiko?}

Die Herausforderung des Verwaltungsrechts bei der Risikoregulierung ist weniger das Ergebnis neuer technischer Entwicklungen, sondern falscher Zurechnungen auf die Gesellschaft oder die Naturwissenschaft und institutioneller Verschiebungen bei der Formulierung und Umsetzung dieser Zurechnungen. Naturwissenschaftliche oder gesellschaftstheoretische Rationalitäten können auf diese Weise nicht in das Recht inkorporiert werden, und andere Rationalitäten, etwa die politische und die ökonomische werden dadurch vernachlässigt. Die rechtliche Regelung von Risiken sollte sich statt dessen an zwei Strategien orientieren. Sie muss sich erstens vom Möglichkeitsdenken verabschieden und die Wahrscheinlichkeit zum Regelungsgegenstand nehmen. Und sie muss zweitens die Zurechnung ungewisser Wahrscheinlichkeit relationieren und individualisieren anstatt sie zu naturalisieren oder zu vergesellschaften. Nur durch individualisierende Zurechnungen kann die soziale Erwartung nach „Risikosteuerung“ rechtlich befriedigt werden. Dieses Programm gilt es instrumentell und institutionell auszugestalten.

Nicht die Instrumente oder Institutionen unterliegen einem Reformbedarf, sondern die kognitiven Zurechnungen. So manche Rechtsentwicklung der letzten Jahrzehnte, die gerade das Ergebnis gesellschaftlicher Kollektivierung oder technischer Naturalisierung ist, sollte daher kritisch überdacht werden. Falschen Regelungserwartungen und einer übertriebenen Expansion des Präventionsgedankens auf utopische Regelungsziele ist entgegenzutreten. ${ }^{152}$

151 Dazu E.-W. Böckenförde Grundrechte als Grundsatznormen, in: ders. Staat, Verfassung, Demokratie, 1991, 159, 163-177; R. Wahl Der Vorrang der Verfassung und die Selbständigkeit des Gesetzesrechts, NVwZ 1984, $401 \mathrm{ff}$.; H. Dreier Dimensionen der Grundrechte, 1993, 10ff.; G. F. Schuppert/C. Bumke Die Konstitutionalisierung der Rechtsordnung, 2000; G. Hermes Verfassungsrecht und einfaches Recht - Verfassungsgerichtsbarkeit und Fachgerichtsbarkeit, VVDStRL 61 (2002), 119, 121-139.

152 Vgl. schon die Skepsis gegenüber dem Präventionsgedanken bei D. Grimm Verfassungsrechtliche Anmerkungen zum Thema Prävention, in: ders. Die Zukunft der Verfassung, 1991, 197, 198, 218: Die Vorteile der Prävention bedingen Nachteile bei der Selbstbestimmung des Einzelnen und der Freiheitlichkeit des Gesamtsystems. „Im Zuge der 
Letzte Sicherheit kann kein rechtliches Ziel sein. Da Risiken nur zugerechnet, nicht aber beseitigt werden können, bleibt immer ein Bereich ungewisser Gefährdungen bestehen. Risiken sind eben die Kehrseite der Freiheit. Eine hypertrophe Sicherheitserwartung darf sich vor allem nicht die Beseitigung der Ungewissheit menschlichen Verhaltens zum Ziel setzen, abgesehen davon, dass sie dies mit wissenschaftlichen Mitteln auch gar nicht erreichen könnte. Wenn jedoch, wie bislang im deutschen Verständnis, der Risikobegriff auch auf mögliche Gefahren abstellt und diese auf die Gesellschaft bezieht, dann enthält er einen gedanklichen Angriff auf die Freiheit des Menschen. Denn eins ist gewiss: Nichts ist so ungewiss wie das Verhalten des Menschen. Daher ist es die vordringlichste Aufgabe eines Risikoverwaltungsrechts zu verhindern, dass der Mensch selbst zum Risiko erklärt wird.

Verhütung einzelner Freiheitsgefahren droht [die präventive Staatstätigkeit] die Freiheitlichkeit der Sozialordnung insgesamt zu schmälern und höhlt gleichzeitig die demokratischen und rechtsstaatlichen Kautelen, die zur Begrenzung der Staatsmacht im Interesse individueller Freiheit entwickelt worden sind, partiell aus." Ebd., 200. 
Leitsätze des 2. Berichterstatters über:

\title{
Risikosteuerung durch Verwaltungsrecht: Ermöglichung oder Begrenzung von Innovationen?
}

\author{
I. Risiko als Zurechnungsproblem: Schadenswahrscheinlichkeit \\ oder Schadensmöglichkeit?
}

(1) Risiko stellt das Recht vor ein prinzipielles Problem: Gefahren werden nicht mehr individuell zugerechnet, sondern kollektiviert. Sie sind nicht mehr Ergebnisse von Handlungen, sondern von sozialen, technischen oder kognitiven Prozessen.

(2) Die Leistung des Risikobegriffs ist es, höchst unterschiedliche Fragestellungen als Problem komplexer Kausalität zu verallgemeinern und als disziplinenübergreifendes Erkenntnisproblem zu formulieren.

\section{Risikobegriffe in der Rechtswissenschaft}

(3) In der Rechtswissenschaft wurde das Zurechnungsproblem in erster Linie in Abgrenzung zum Gefahrenbegriff thematisiert. Bei der Abgrenzung von Risiko und Gefahr konkurrieren zwei Grundverständnisse, die entweder auf die Schadenswahrscheinlichkeit oder die Schadensmöglichkeit abstellen.

a) Dreistufenmodell: Abgrenzung nach Wahrscheinlichkeit

(4) Das Dreistufenmodell grenzt Gefahr, Risiko und Restrisiko nach dem Grad der Wahrscheinlichkeit ab. Risiko bleibt ein normativ undefinierter Bereich unwahrscheinlicher Gefahren.

\section{b) Zweistufenmodell: Abgrenzung nach Möglichkeit}

(5) Nach dem Zweistufenmodell bleiben Gefahren auf die Wahrscheinlichkeit des Schadenseintritts begrenzt, während der Risikobegriff auf die Möglichkeit des Schadens bezogen wird. Dadurch kann ein vom Gefahrenbegriff unabhängiger Begriff des Risikos gewonnen werden. Dieser auf ungewisse Schadensmöglichkeiten bezogene Risikobegriff ist in den 1990er Jahren entstanden und hat sich in der Literatur inzwischen durchgesetzt. 
2. Risikobegriffe in der Gesetzgebung

a) Der Wandel vom wahrscheinlichen zum möglichen Schaden im deutschen Recht

(6) Der Wandel vom Wahrscheinlichkeitsbezug zum Möglichkeitsdenken lässt sich auch in der Gesetzgebung nachvollziehen. Das Gentechnikgesetz ist das legislatorische Dokument des Wandels zum Zweistufenmodell.

b) Die Rückkehr zum wahrscheinlichen Schaden im Europarecht

(7) Die Rechtslage im Europarecht ist durch die Rückkehr zur Abgrenzung nach Wahrscheinlichkeitsgraden gekennzeichnet.

c) Auswirkung auf das Verständnis des Vorsorgeprinzips

(8) Die Differenzen zwischen deutschem und europäischem Recht wirken sich auf die nähere Bestimmung des Vorsorgeprinzips aus. Vor dem europarechtlichen Hintergrund ist das deutsche Zweistufenmodell ein Auslaufmodell, so dass die rechtswissenschaftliche Erwartungshaltung in Deutschland auf mehr gerichtet ist, als der europarechtliche Risikobegriff einlösen wird.

\section{Risiko als ungewisse Möglichkeit}

(9) Die Möglichkeit eines Schadenseintritts kann kein Gegenstand rechtlicher Regelungen sein. Ein solches Risikoverständnis erzeugt illusionäre Erwartungen und muss aufgegeben werden.

\section{Verhalten statt Technik als Regelungsgegenstand}

(10) Gegenstand des Rechts kann nicht die Technik selbst, sondern nur der Umgang des Menschen mit ihr sein. Wollte das Recht die Möglichkeit von Gefahren ausschließen, müsste es die Möglichkeit freien menschlichen Verhaltens ausschließen.

(11) Selbst ein Forschungs- und Anwendungsverbot wäre zum Ausschluss möglicher Gefahren ungeeignet. Ob sich die Normadressaten tatsächlich an die normative Erwartung halten, obliegt der freien Entscheidung jedes Einzelnen. Jedes normgemäße Verhalten ist ein individueller Entschluss, auf den das Recht Einfluss nimmt, den es aber nicht ersetzt.

2. Subjektive statt objektiver Erkennbarkeit technischer Ungewissheit

(12) Technik ist kein objektiver Zustand, sondern Ergebnis subjektiver Handlungen. Ihre Wirkungszusammenhänge können nur durch Handlungs- 
erfahrung aufgedeckt werden. Die Schadensmöglichkeit ist daher kognitiv immer nur als Problem der Wahrscheinlichkeit erkennbar. Objektiv gefahrlose Zustände können rechtlich nicht bezweckt werden.

(13) Juristen geben sich als die letzten Vertreter zu erkennen, die der $\mathrm{Na}$ tur eine apriorische Objektivität zubilligen und glauben, das Verständnis der Natur sei ohne subjektive Konstruktionsleistung möglich. Der Risikobegriff ist eine moderne Emanation des klassischen erkenntnistheoretischen Problems, technische Realität und naturwissenschaftliche Kausalität als objektives Sein dem Sollen überzuordnen und dadurch praktisches Handeln zu lähmen.

\section{Experimente und flexibles Erfahrungswissen}

(14) Erst das Experimentieren mit neuen Techniken liefert das nötige Wissen für eine Risikobewertung. Durch Ausschluss möglicher Risiken Sicherheit $z u$ erstreben, ist weder theoretisch noch praktisch sinnvoll. Rechtliche Regelungen müssen einerseits Experimente zulassen, aus ihnen andererseits Erfahrungen ziehen, also individuelle Verhaltensregelungen treffen und sie als Normen verallgemeinern.

(15) Komplexität ist eine Ausgangsbedingung demokratischer Rechtsetzung und nicht Ausdruck einer Zivilisationskrise.

\section{Die Entindividualisierung der Zurechnung}

(16) Die illusionäre Ausdehnung des Regelungsgegenstandes auf die Möglichkeit von Schäden basiert auf zwei Prämissen, die als Naturalisierung und Vergesellschafiung der Zurechnung bezeichnet werden können.

\section{Risiko als Naturalisierung und Vergesellschaftung der Zurechnung}

(17) Technikfolgen werden naturalisiert, wenn sie auf naturwissenschaftliche Wirkungszusammenhänge bezogen werden, die individuellen Handlungen vorausliegen sollen. Umweltschäden werden vergesellschaftet, wenn die zeitversetzten, kumulierenden und grenzüberschreitenden Effekte als ein gesellschaftliches Phänomen begriffen werden, zu dem alle beitragen. Die Zurechnung von Ungewissheit verändert sich: von der subjektiven zur objektiven Beherrschbarkeit, vom Einzelnen zum Kollektiv.

(18) Mit der naturalisierten oder vergesellschafteten Zurechnung von Risiken wird die Abkehr von einer akteurszentrierten Rechtsordnung in Kauf genommen. Dies führt zu erheblichen Folgeproblemen. Die Diskussion um den Risikobegriff hat eine verfassungsrechtliche Dimension, die um die 
Stellung des handelnden Subjekts in der Rechtsordnung kreist. Die Debatte ist Ausdruck einer kognitiven Verschiebung vom Einzelnen auf die Gesellschaft.

\section{Rezeption sozialwissenschafilicher Problemwahrnehmungen}

(19) Die Begriffe „Risiko “ und "Steuerung“ sind terminologische Transmissionsriemen, mit denen technische und gesellschaftiche Erwartungshaltungen disziplinenübergreifend gebündelt werden. Auch unter dem Eindruck einer sozialwissenschafilichen Öffnung der Rechtswissenschaft sollten solche Erwartungshaltungen nicht zu einem kognitiven und dogmatischen Auftrag des Verwaltungsrechts erklärt werden, zumal die gesellschaftstheoretische Problemformulierung des Risikos weder für die Rechtswissenschaft verpflichtend noch in den Sozialwissenschaften unumstritten ist.

3. Rückkehr zu individualisierenden Zurechnungen als soziale Leistung des Rechts

(20) Der Regelungsanspruch muss von der Ungewissheit der Möglichkeit auf die Ungewissheit der Wahrscheinlichkeit und von einer kollektiven $\mathrm{Zu}$ schreibung auf eine subjektive Zurechnung reduziert werden. Andernfalls würden soziale oder technische Erwartungen geweckt, die das Recht schon theoretisch nicht erfüllen kann und die praktische Enttäuschungen hervorrufen müssen.

IV. Risiko als ungewisse Wahrscheinlichkeit

(21) Die Aufgabe des Risikoverwaltungsrechts kann nur die individuelle Zurechnung ungewisser Wahrscheinlichkeit sein.

\section{Relations- und Selektionsbedürfigkeit}

(22) Wahrscheinlichkeit kann nicht absolut, sondern immer nur im Verhältnis verschiedener Parameter bestimmt werden. Relationen müssen juristisch ermittelt werden, damit Wahrscheinlichkeitsurteile überhaupt getroffen werden können. Das Recht trägt durch Zurechnungen zur Beseitigung faktischer Ungewissheit bei. 
2. Normative Nachverdichtung der offenen Relationierung des Gefahrenbegriffs

(23) Das Grundmuster einer Relationierung enthält der Gefahrenbegriff des Polizeirechts. Die Gefahrenabwehrdogmatik lässt alle Parameter des Wahrscheinlichkeitsurteils offen und kann erst durch den Bezug zu den Fakten des Einzelfalls relationiert werden. Gerade weil der offene Gefahrenbegriff potentiell aufjede faktische Relation anwendbar ist, ist er auf ungewisse Wahrscheinlichkeiten nicht anwendbar, wenn diesen die Konkretisierbarkeit des Einzelfalls fehlt.

(24) Nur aus faktischen Gründen genügt der Gefahrenbegriff nicht zur Bewältigung von Risiken. Normative Bewertungen müssen die Funktion der faktischen Konkretisierung übernehmen. Die Lösung liegt nicht in der prinzipiellen Abkehr von der ordnungsrechtlichen Gefahrenabwehrdogmatik, sondern in ihrer normativen Nachverdichtung.

3. Instrumentelle Umsetzung auf der Ebene des einfachen Rechts

a) Adressatentypisierung, Handlungstypisierung, Erfolgstypisierung

(25) Die materielle Nachverdichtung kann bei der Normativierung eines der drei offenen Parameter des Gefahrenbegriffs ansetzen: des Störers, der Kausalbeziehung und des Rechtsguts. Hierbei kann zwischen der Adressatentypisierung, der Handlungstypisierung und der Erfolgstypisierung unterschieden werden.

\section{b) Instrumentenvielfalt}

(26) Je genauer die Parameter normativiert werden, desto leichter lassen sich ungewisse Wahrscheinlichkeiten ermitteln und bewerten. Erfolgstypisierungen und Adressatentypisierungen sind dafür besser geeignet als Handlungstypisierungen, weil die Vielfalt menschlicher Verhaltensmöglichkeiten eine entsprechend umfangreiche Normproduktion erforderte.

(27) Ziel muss es immer sein, Risiken zu individualisieren. Dabei empfiehlt es sich, unterschiedliche Zurechnungen vorzunehmen, um möglichst viele Wahrscheinlichkeitsrelationen normativ zu erfassen.

\section{c) Unzweckmäßige Relationierungen}

(28) Nicht wenige Gesetze werden dem Anspruch an eine normative Nachverdichtung nicht gerecht. Bedenklich erscheint eine Ausrichtung am Leitbild des Verursacherprinzips, denn dieses fördert einseitig Handlungszurechnungen und privilegiert damit die regelungstechnisch schwierigste Relationierung. 
4. Institutionelle Umsetzung auf der Ebene des Verfassungsrechts

a) Umsetzung als juristische Rationalität individuell-konkreter Rechisetzung

(29) Momentan wird Risikoregulierung institutionell in erster Linie als Auftrag der Verwaltung begriffen. Wenn die Lösung aber nur im Wege der abstrakt-generellen und nicht der individuell-konkreten Normsetzung zu finden ist, wird Risiko als Rechtsproblem an der falschen Stelle institutionalisiert.

(30) Auch die sozialen Aspekte des Problems werden institutionell falsch verortet, wenn man bei der individuell-konkreten Rechtsetzung die Vergesellschafiung durch die Einbeziehung partizipativer gesellschafticher Teilhabe und die Naturalisierung durch die Delegation an Experten lösen will.

\section{b) Umsetzung als politische Rationalität abstrakt-genereller Rechtsetzung}

(3I) Weil sich Risiko als Rechtsproblem nur mit abstrakt-genereller Rechtsetzung bewältigen läßt, handelt es sich um ein politisches Problem, das institutionell die kognitiven Fähigkeiten des Parlaments erfordert. Der politische Prozess vermag die faktischen Voraussetzungen für eine normative Relationierung zu erbringen und vermittelt die subjektiv unterschiedliche Wahrnehmung von Risiken.

(32) Die richtige Rechtsform gesellschafilicher Lernfähigkeit ist die abstrakt-generelle Normsetzung. Neben dem Parlamentsgesetz ist auch die Verordnungsgebung als eine Handlungsform des politischen Prozesses zu verstehen. Sie sollte nicht zu stark verrechtlicht werden, sonst kann sie ihre Aufgabe zur flexiblen und schnellen Normierung nicht mehr wahrnehmen.

(33) Nicht nur der Gesetzesvorbehalt, sondern auch die Delegationsschranken bedürfen im Hinblick auf die Erkenntnisleistungen der Institutionen stärkerer Konturierung.

(34) Durch die Konstitutionalisierung gesetzlicher Inhalte oder die Verrechtlichung politischer Formen findet eine Verrechtlichung am falschen Platz statt, weil der Rationalität politischer Institutionen zu wenig zugetraut und das Rechtsstaatsprinzip über das Demokratieprinzip gestellt wird.

\section{Der Mensch als Risiko?}

(35) Falschen Regelungserwartungen und einer übertriebenen Expansion des Präventionsgedankens auf utopische Regelungsziele muss entgegengetreten werden. Da Risiken nur zugerechnet, nicht aber beseitigt werden können, bleibt immer ein Bereich ungewisser Gefährdungen bestehen. Letzte Sicherheit kann kein rechtliches Ziel sein. 
(36) Nichts ist so ungewiss wie das Verhalten des Menschen. Daher ist es die vordringlichste Aufgabe eines Risikoverwaltungsrechts zu verhindern, dass der Mensch selbst zum Risiko erklärt wird. 


\section{Aussprache und Schlussworte}

\section{Risikosteuerung durch Verwaltungsrecht: Ermöglichung oder Begrenzung von Innovationen?}

Vorsitzender (Schuppert): So, meine Damen und Herren, lassen Sie uns ungesäumt mit unserer Diskussionsrunde beginnen. Wir haben - wenn ich es recht sehe - vor allem drei Problemgruppen zu diskutieren. Die erste und - was nicht überraschen kann - umfänglichste Problemgruppe bezieht sich auf den Risikobegriff, also die Abgrenzung von Risiko und Gefahr, von Wahrscheinlichkeit und Risiko und all das. Nach den dazu vorliegenden 11 Wortmeldungen werden wir hoffentlich alle wissen, was es mit dem Risikobegriff auf sich hat. Dann gibt es drei Wortmeldungen zum Problem der Konstituierung eines Risikoverwaltungsrechts sowie eine weitere zum Stichwort „Risikopsychologie“. Die letzte Fallgruppe rangiert unter dem Begriff „Verschiedenes“; darunter sind diejenigen Wortmeldungen zu finden, die auf den ersten Blick nicht ohne weiteres zuzuordnen waren. Ich finde es richtig, wenn wir mit einem gestandenen Polizeirechtler beginnen und deswegen wird Herr Denninger eröffnen.

Denninger: Meine sehr verehrten Damen und Herren, ich habe heute Morgen mit großer Aufmerksamkeit zwei hervorragende Referate gehört, die zum Weiterdenken auffordern und eigentlich zwingen. Nach dem und während des Referates von Herrn Scherzberg habe ich mir überlegen müssen, ob ich nicht meinen Abschied als Polizeirechtler - sozusagen als ein mittelalterlicher Dinosaurier - nehmen müsse. Herr Lepsius hat mich dann mit seiner These 12, dass es zwecklos sei, gefahrlose $\mathrm{Zu}$ stände rechtlich zu bezwecken, von dieser Sorge und Furcht wieder erlöst. Und ich glaube, so gegensätzlich die beiden Referate zunächst scheinen, sie handeln von identischen Problemen, bedienen sich aber ganz unterschiedlicher Sprachen, und wir müssen, glaube ich, das in der Diskussion sehr genau berücksichtigen. Herr Lepsius hat die klassische, wenn ich so sagen darf, Sprache „akteurszentrierter“ Rechtsbetrachtung, also handlungsorientiert, gewählt, Herr Scherzberg hat, eher systemtheoretisch orientiert, eine Sprache des Technikverständnisses gewählt. Das nur zum allgemeinen Hintergrund, der es auch ermöglicht, die Brücke zu schlagen zu dem Thema des gestrigen Nachmittags: „Freiheit und Sicherheit", die, meine ich, hier geschlagen werden muss. Sie hat sich bei mir in 
der Fragestellung konzentriert: Wie ist es denn, wenn technische Risiken von extremen Handlungsrisiken begleitet werden beziehungsweise wenn beide kombiniert werden, zum Beispiel durch selbstmordentschlossene Terroristen, die Massenvernichtungsmittel irgendeiner Art einsetzen? Da kommen sozusagen beide Ströme der Technikrisikosteuerung und der Handlungsrisikosteuerung zusammen. Also große Zustimmung zu den Ausführungen von Herrn Lepsius, insbesondere finde ich es richtig und notwendig, dass er die verfassungsrechtliche Dimension hier hervorgehoben hat. Die Individualisierbarkeit der Zurechnung, wenn ich das als eine kleine Ergänzung anmerken darf, zeigt sich ja spätestens auch bei der judiziellen Bewältigung solcher Konflikte, denn die Richter haben ja letztlich dann immer über Anträge, die sie ablehnen oder genehmigen, beziehungsweise über Strafsanktionen und Ähnliches zu entscheiden. Also irgendwo muss die Rechtsordnung dann die Kurve von der Steuerung wieder zur individuellen Zurechnung bekommen. Und das ist Ihnen, Herr Lepsius, hervorragend gelungen, und dafür möchte ich Ihnen danken. Ein letztes Fragezeichen aber, von vielen kleineren Fragezeichen abgesehen: Bitte überdenken Sie doch Ihren Begriff der „ungewissen Wahrscheinlichkeit" noch einmal. Also, ich kann mir unter "wahrscheinlich“ etwas vorstellen und ich kann mir unter „unwahrscheinlich“ etwas vorstellen, aber eine "ungewisse Wahrscheinlichkeit“ ist wahrscheinlich eine sprachliche Verkürzung in der Weise, dass sich die Ungewissheit auf Elemente dessen, was die Wahrscheinlichkeit ausmacht, bezieht, und dann sind wir doch wieder bei der Graduierung des Wahrscheinlichkeitsbegriffs.

Lege: Es fällt natürlich schwer, angesichts der Komplexität dieser Referate eine genaue Frage zu stellen. Ich habe trotzdem eine Ergänzung zu Ihnen, Herr Scherzberg, und eine sehr grundlegende Frage an Sie, Herr Lepsius. Zunächst glaube ich allerdings, dass Sie beide sich in den praktischen Konsequenzen durchaus unterscheiden, und zwar in der Frage, wenn ich das so plakativ sagen darf, wem der Schwarze Peter für bestehende Ungewissheiten zugeschoben werden soll. Ich glaube, da unterscheiden Sie sich sogar ganz gewaltig.

$\mathrm{Zu}$ Ihnen, Herr Scherzberg: Sie haben sehr schön unterschieden zwischen dem Risiko erster und zweiter Ordnung. Das Risiko erster Ordnung ist die Höhe der Wahrscheinlichkeit des Schadens, das Risiko zweiter Ordnung die Gefahr der Fehleinschätzung dieser Gefahr. Nun sind Sie aber Systemtheoretiker genug um zu wissen, dass damit die Sache nicht zu Ende ist. Es gibt sicherlich auch noch Risiken dritter und vierter Ordnung. Insofern fiel mir bei Ihren Ausführungen spontan ein: Ein Risiko dritter Ordnung sind zum Beispiel die Chancen und Risiken, die sich 
mit den gewählten Mitteln der Risikovorsorge verbinden - etwa mit Monitoring oder EMAS, die Sie genannt haben. Ich erinnere mich an ein $\mathrm{Ge}-$ spräch in Dresden mit den Kollegen aus der Biologie, das ich im Zusammenhang mit dem Gentechnikrecht geführt habe. Diese Kollegen sagten mir zunächst einmal, dass die administrative Kontrolle ihrer gentechnischen Arbeiten sich nach einiger Zeit gut eingespielt hatte. Das administrative Wissen hatte sich so weit konsolidiert, dass es keine Probleme mehr gab. Dann aber wurde an der TU Dresden, erstmals übrigens an einer Universität, ein EMAS-Verfahren durchgeführt, und seither gibt es damit große Probleme, die gar nicht unbedingt mit der Gentechnik zusammenhängen, sondern mit vielen anderen Dingen. Offensichtlich wird also durch das EMAS-Verfahren gleichsam Ungewissheit an die Akteure zurückgegeben. Sie bekommen den Schwarzen Peter, dessen Effekt vor allem eines ist, nämlich bremsen, und genau darin besteht ein Risiko dritter Ordnung. Mit anderen Worten: Die Risiken erster, zweiter, dritter, vierter und so weiter Ordnung steigern sich zu immer höherer Komplexität, und dann stellt sich für jeden, der dennoch handeln will oder muss, die Frage: Wo zieht man die Grenze? Bei Ihnen habe ich einen Ansatz vermisst, wie das Recht diese Erkenntnisproblematik handhaben kann oder soll. In der Tendenz habe ich es so empfunden, dass Sie das Risiko jeglicher Ungewissheit auf diejenigen abschieben wollen, die als Akteure Risiken verursachen könnten.

$\mathrm{Zu}$ Herrn Lepsius: Mir ist eines nicht klar geworden, und das sind Ihre beiden Grundbegriffe „Wahrscheinlichkeit“ und „Möglichkeit“. Was ist der Unterschied? Es könnte ein quantitativer Unterschied sein oder ein qualitativer Unterschied, und ich denke, Sie verstehen Möglichkeit quantitativ als ein Minus zur Wahrscheinlichkeit. Demgegenüber meine ich: „Wahrscheinlichkeit“ ist ein erfolgsbezogenes Kriterium, während „Möglichkeit" an das Handeln anknüpft. Deshalb muss ich Ihren Thesen 21 und 28 widersprechen, vor allem These 21: „Die Aufgabe des Risikoverwaltungsrechts kann nur die individuelle Zurechnung ungewisser Wahrscheinlichkeit sein". Nein, Zurechnung ist die Zurechnung von Handeln, hier: von Handeln trotz Ungewissheit. Und da habe ich Sie dann so verstanden, dass Sie den Schwarzen Peter für diese Ungewissheit eher der Gesellschaft zuschieben wollen als den Akteuren.

Schwabe: Einen Einwand hat eben Herr Lege vorweggenommen. Auch ich habe eine präzise Umschreibung der Wahrscheinlichkeit und damit eine Trennung zwischen Wahrscheinlichkeit und Möglichkeit vermisst. Das wäre also etwas, was noch nachzuholen wäre. Angesichts der großen Bedeutung dieser beiden Begriffe möchte ich nochmals hinweisen auf den meines Erachtens absurden Umgang, den wir - freilich schon seit 
Jahrzehnten - mit dem Begriff der Wahrscheinlichkeit pflegen. Selbst Risikopotentiale von 1:100000 werden ja bei uns noch eingefangen unter die Gefahr und damit unter die Wahrscheinlichkeit. Ich verstehe nicht, wie wir hier bei diesem Begriff der Gefahr und der Wahrscheinlichkeit uns vom allgemeinen Sprachgebrauch so absondern konnten, wie das die ganze Zeit und ohne irgendwelche Proteste und Einwände (außer von mir gelegentlich) geschieht. Meine Damen und Herren, wenn wir uns am Samstag verabschieden, und Sie fahren nach Hause, und ich sage Ihnen „wahrscheinlich werden Sie auf der Autobahn verunglücken“, dann werden Sie mich für meschugge erklären. Ich bin aber nicht meschugge, einfach deswegen, weil Sie sicherlich auf der Autobahn der Gefahr eines Unfalls ausgesetzt sind und wir Gefahr mit Wahrscheinlichkeit definieren: Also muss ich doch sagen dürfen ,wahrscheinlich werden Sie verunfallen“. Und jetzt ahne ich, wie Sie sich aus der Schlinge ziehen wollen, Sie wollen nämlich sagen: „Wir sprechen nicht nur von Wahrscheinlichkeit, sondern von hinreichender Wahrscheinlichkeit“. Das lasse ich nicht gelten, einfach deshalb, weil ein Adjektiv einen Begriff nicht denaturieren kann, und ich begründe das so, dass nach meinem Sprachempfinden jetzt eben Tag ist, und nicht etwa eine nicht hinreichende oder gerade noch hinreichende Nacht.

Mager: Meine sehr geehrten Damen und Herren, auch ich habe ein Frage zu der für Ihren Vortrag, Herr Lepsius, grundlegenden Unterscheidung zwischen Wahrscheinlichkeit und Möglichkeit. Mir haben insoweit die Definitionen am Anfang gefehlt. Bei dem Begriff Möglichkeit denke ich gleich an die Klagebefugnis. Die für die Klagebefugnis geforderte Möglichkeit der Rechtsverletzung wird in ständiger Rechtssprechung definiert als eine Rechtsverletzung, deren Vorliegen nicht von vornherein ausgeschlossen sein ist. „Nicht von vornherein ausgeschlossen“ ist aber auch ein Grad der Wahrscheinlichkeit. Bei dem Begriff der Wahrscheinlichkeit denke ich natürlich gleich an den Gefahrenbegriff. Wie Herr Schwabe eben schon sagte, ist für den Gefahrenbegriff die hinreichende Wahrscheinlichkeit entscheidend. Dieser wird bestimmt nach der, ,je-desto"-Formel: je größer der zu erwartende Schaden, umso geringer die Anforderung an die Möglichkeit des Schadenseintritts. Also auch hier sind Wahrscheinlichkeit und Möglichkeit aufeinander bezogen. Ähnlich wie auch schon Herr Lege gefragt hat, wüsste ich gerne: Meinen Sie, dass zwischen Wahrscheinlichkeit und Möglichkeit nur ein gradueller Unterschied besteht und wollen Sie nur eine Neuausrichtung der Perspektive in Bezug darauf, welcher Grad bzw. welche Grade auf dieser Skala rechtliche Bedeutung entfalten? Oder meinen Sie einen kategorialen Unterschied? 
Breuer: Wir haben heute Morgen zwei ausgezeichnete, sich hervorragend ergänzende Referate gehört. Im Grundsätzlichen bin ich zur Zustimmung geneigt; was konkret aus den Referaten folgt, möchte ich mit einigen Nachfragen nach Möglichkeit noch deutlicher werden lassen. Zustimmen möchte ich Herrn Scherzberg bei seiner Situationsanalyse. In der Tat besteht das Kernproblem darin, wie wir mit der Ungewissheit umgehen. Zustimmen möchte ich Herrn Lepsius insoweit, als er von einem allzu weit gespannten Möglichkeitsdenken wegkommen will. Ich meine, darin die Aussage verstanden zu haben, dass wir uns wieder bemühen sollten, zu differenzieren und nicht in einem allzu weit ausgreifenden Denken illusionärer Forderungen gefangen zu bleiben. Zwei Fragenkreise knüpfen sich für mich daran an. Der erste Fragenkreis kann unter das Thema gestellt werden: Was folgt für das Verwaltungsrecht daraus? Das Verwaltungsrecht muss Kompetenzen und Entscheidungsbefugnisse festlegen, es muss Rechte und Pflichten der Beteiligten bestimmen. Diese Frage ist, jedenfalls traditionell, im deutschen Umwelt- und Technikrecht an der Begriffstrias der Gefahr, des Risikos und des Restrisikos festgemacht worden. Wo es um Gefahren geht, ist absoluter Schutz geboten; dabei kann man auch auf das gestrige Thema zurückgreifen, Freiheit und Sicherheit sind letztlich grundrechtliche und rechtsstaatliche Postulate. Im Bereich des Risikos handelt es sich um eine offene Abwägung, so dass bestimmt werden muss, wer im demokratischen Rechtsstaat befugt ist, hierüber die Entscheidung zu treffen. Das Restrisiko gehört einfach zu unserer Welt, und hier spricht in der Tat vieles dafür, dass wir Optionsspielräume Privater anerkennen sollten. Insoweit waltet dann die private Freiheit, und der Staat hat bestenfalls einen ordnungsrechtlichen Rahmen zu setzen. Mir wäre daran gelegen, noch mehr Klarheit zu bekommen, was in dem institutionalisierten System der parlamentarische Gesetzgeber zum Grundrechts- und Freiheitsschutz zu bestimmen hat, was die Verwaltung aufgrund gesetzlicher Ermächtigung und in Wahrnehmung einer Risikovorsorge so oder anders bestimmen kann und was schließlich der privaten Freiheit dort überlassen ist, wo nur Restrisiken anstehen. Insbesondere ist das Stichwort „Optionsspielräume Privater", das ich bei Herrn Scherzberg vernommen habe, ein interessanter Ansatz. Auch die Öffentlichrechtler sollten zur Kenntnis nehmen, dass es nicht nur staatliche Institutionen gibt, sondern in der freien Gesellschaft Individuen eigenverantwortliche Entscheidungen zu treffen haben.

Zweiter Fragenkreis: Mich bewegt der Unterschied des Risikodenkens auf der deutschen und auf der europäischen Ebene. Im deutschen Recht sind wir es gewohnt, vom Ordnungsrecht auszugehen, von Rechten und Pflichten, insbesondere Betreiberpflichten, von technischen Standards, insbesondere Emissionsstandards; damit sind die wesentlichen Vorteile 
im innerstaatlichen Umweltrecht erzielt worden. Im europäischen Recht treten mehr und mehr holistische Postulate in den Vordergrund: Der integrierte Umweltschutz wird verlangt, ferner die Umweltverträglichkeit und die Umweltqualitätsziele. Hier liegen gewaltige Unterschiede, wenn wir versuchen, die Differenz praktisch zu erfassen. Die Europäische Gemeinschaft nimmt mehr und mehr Abschied von Emissionsgrenzwerten und strikten technischen Anforderungen, und auf der anderen Seite haben wir große Schwierigkeiten, diese holistischen Postulate überhaupt noch in das nationale Recht aufzunehmen. Wo bisher zur Luftreinhaltung Emissionsgrenzwerte verlangt werden, sind diese mehr und mehr in den Hintergrund getreten. Mir wäre an einer Klarstellung gelegen, ob gerade Ihren Prinzipien, Herr Lepsius, diese holistischen Postulate gerecht werden oder ob Sie dies verneinen würden.

Ladeur: Ich möchte auch etwas sagen zum Gefahrenbegriff. Zunächst zu Herrn Scherzberg: Mir scheint, dass man doch noch stärker betonen muss, dass der Gefahrenbegriff mit einem bestimmten gesellschaftlich begründeten Wissensverständnis, einer Wissensbasis verknüpft ist, und das Besondere daran ist, dass sie eben gemeinsam, sowohl von den Privaten als auch von der Verwaltung/Polizei genutzt werden kann, was die Anwendbarkeit vereinfacht. Damit schwingen natürlich immer auch Wertungen mit, die ebenfalls geteilt werden von der Verwaltung und der Öffentlichkeit, über die dann nur noch im Einzelnen gestritten wird, nicht aber grundsätzlich. Mir scheint ganz wichtig zu sein, dass dort inzwischen ein Bruch eingesetzt hat. Es gibt nicht mehr diese früher gemeinsamen Überzeugungen und die geteilte Wissensbasis. Dahin gibt es auch kein Zurück mehr. Und man muss sich stärker darum bemühen, nach funktionalen Äquivalenten zu suchen: Gibt es nicht eine neue gemeinsame Wissensbasis, die geeignet ist, die Risiken, die sich eben jenseits der Erfahrung und jenseits des gemeinsamen Erfahrungswissens bilden, zu beschreiben und zu bewerten? Dies eine ganz wichtige Frage, und sie lässt sich nach meiner Ansicht nur durch Höherlegung des Abstraktionsniveaus, durch Rekurs auf ein neues gemeinsames Methodenverständnis, aufnehmen. Und da würde ich - um ein paar Argumentationsschritte zu überspringen - doch meinen, dass man eine Lanze brechen sollte für die quantitativen Methoden, weil sie vielleicht geeignet sein könnten, eine neue von Privaten wie vom Staat zu nutzenden gemeinsame Wissensbasis zu stiften. Und dies trotz aller Einwände, die dagegen erhoben werden etwa, dass natürlich immer normative Annahmen mitschwingen, was aber früher, ja auch früher, der Fall war. Zu fragen ist aber, ob es nicht sinnvoller ist, gewisse Faktoren zu quantifizieren, gerade im Hinblick auf die Vergleichbarkeit von Risiken, und zwar auch wenn man sich immer 
darüber verständigen muss, dass man natürlich da mit durchaus fiktiven, hypothetischen Annahmen rechnet - aber die Vergleichbarkeit ist dann sehr viel stärker gewährleistet. Das wäre sozusagen ein funktionales Äquivalent zu der früher gemeinsamen Erfahrung, zu der eine Vielzahl von Unternehmen zum Beispiel beigetragen haben. Dann zu Herrn Lepsius, das schließt sich direkt daran an: Mir scheint, dass die Annahme etwas zu kurz gegriffen wäre, anzunehmen, dass der Unterschied zwischen dem Gefahrenbegriff und dem Risikobegriff sich an der Einzelfallorientierung festmachen lässt. Das glaube ich gerade nicht: Auch der Gefahrenbegriff greift auf die allgemeine Erfahrung zurück, die dann nur auf den Einzelfall bezogen wird, aber die Einzelfallrelation macht ihn gerade nicht aus, und deshalb ist es auch sehr zweifelhaft, ob es ein gangbarer Weg ist, eine normative Konkretisierung des Gefahrenbegriffes über den Gesetzgeber erreichen zu wollen. Ich glaube, es führt kein Weg daran vorbei, diese doch eben über außerparlamentarische Expertise zu bewältigen, die dann ein funktionales Äquivalent zur Herstellung gemeinsamen Erfahrungsbasis liefern müsste.

Murswiek: Herr Lepsius hat einen eindrucksvollen rhetorischen Kampf gekämpft. Er hat allerdings gegen einen Gegner gekämpft, den ich in der Realität unserer Rechtsordnung und der wissenschaftlichen Auseinandersetzung der letzten zwanzig Jahre nicht zu erkennen vermag. Gegen einen Popanz, den er sich für die Zwecke seines Referats konstruiert hat. Natürlich, Herr Lepsius: Es ist unvernünftig, den Ausschluss jedes Risikos rechtlich zu verlangen, es ist unvernünftig, die Ungewissheit beseitigen zu wollen. Aber es gibt kein einziges Gesetz, das den Ausschluss jedes Risikos verlangt, und es gibt, soweit ich weiß, keinen unter den Fachkollegen des Umweltrechts, keinen Autor der wissenschaftlichen Literatur, der dies will. Und dieses Vorbeireden an der Realität des Rechts und der rechtswissenschaftlichen Diskussion spiegelt sich auch in Ihren Begriffsbildungen wider. Wenn Sie als eine zentrale Gegenüberstellung uns vorführen einerseits die Möglichkeit des Schadenseintritts, andererseits die Wahrscheinlichkeit, und wenn Sie Risiko und Gefahr entsprechend zuordnen, dann verkennen Sie, dass es einerseits sehr unterschiedliche Wahrscheinlichkeitsgrade gibt und Gefahren Risiken mit einer ,hinreichend großen" Wahrscheinlichkeit sind, dass es andererseits aber oft nicht möglich ist, den Wahrscheinlichkeitsgrad annähernd zuverlässig zu ermitteln. Die Annahme, dass derjenige, der Risiko mit der Möglichkeit des Schadenseintritts definiert, verlangen würde, es müsste jeder Ausschluss der Möglichkeit eines Schadens gefordert werden, ist eine unzulässige Unterstellung. Risiko ist insofern nur eine beschreibende Kategorie. Welche rechtlichen Folgerungen daran geknüpft werden, das ist dann 
eine zweite Sache. Herr Scherzberg hat sehr schön gezeigt, dass es dazu einen großen Variantenreichtum von Möglichkeiten gibt: vom Ausschluss von Gefahren als hinreichender Wahrscheinlichkeit eines Schadenseintritts angefangen, bis hin zu verschiedenen Möglichkeiten der Vorsorge, der Risikoverminderung, und Risikovermeidung; das ist etwas anderes als Ausschluss jeden Risikos. Bei Ihnen, Herr Lepsius, hat es den Eindruck erweckt, als ob es immer nur diese Ja-Nein-Alternative, entweder Bekämpfung einer Gefahr oder eines Risikos oder überhaupt nichts gebe, aber dazwischen gibt's doch die Möglichkeit der Minderung, und darauf sind Sie nicht eingegangen.

Noch eine Bemerkung zur Individualisierung des Risikos, die Sie gefordert haben: Sie haben gesagt, das Risiko sei eigentlich ein Problem nur wegen der Kollektivierung, die man vorgenommen habe. Ich halte das für falsch unter zwei Aspekten. Einerseits: Individuell zurechenbare Risiken - im Unterschied zu Gefahren - waren schon lange ein großes Problem, wenn Sie etwa an die Risiken der Atomenergie denken, die individuell zugerechnet werden können, aber wo Risikoreduzierung auch unterhalb der Schwelle der auf das Individuum bezogenen Gefahr geboten sein kann. Auf der anderen Seite: Denken wir an die globalen Umweltprobleme wie etwa Klimaschutz. Zu versuchen, den Beitrag jedes Einzelnen zur Entstehung des Ozonlochs ausrechnen zu wollen, könnte zur Bewältigung des Problem nichts beitragen. Das Risikoproblem ist hier als solches ein Kollektivproblem. Gebote und Verbote zur Bewältigung eines solchen Problems müssen dann freilich individuell sein, aber das schließt doch nicht aus, das Risiko bzw. die Gefahr kollektiv (also unabhängig von der Größe des individuellen Verursachungsbeitrags und von der Größe des individuellen Schadenspotentials) zu verstehen.

Ronellenfitsch: Herr Scherzberg, ich möchte Sie beglückwünschen, dass Sie fast jeden Ausdruck der postökologischen Epoche aufgegriffen, einige neue dazugedichtet und das $\mathrm{zu}$ einem System verdichtet haben. Wir werden das diskutieren, wenn diese Epoche - so schätzungsweise in drei Jahren - zu Ende sein wird; es sei denn, die Umwelt kommt in Form einer Flut ihren Schützern wieder zugute. Herr Lepsius, bei Ihnen möchte ich mich bedanken, dass Sie die Aufmerksamkeit wieder auf den Gefahrenbegriff zurückgelenkt haben. Sie haben sich jedoch eine offene Flanke eröffnet durch die Möglichkeit und die Eintrittswahrscheinlichkeit. Wenn Sie das formal betrachten - wenn Sie die Möglichkeit und die Eintrittswahrscheinlichkeit als Quotient betrachten - wenn also auch die Möglichkeit im Zähler eine eins hat, gelangen Sie zur Gewissheit, also gibt es keinen kategorialen Unterschied zwischen Eintrittswahrscheinlichkeit und Möglichkeit. Trotzdem haben Sie Recht: Die Wahrscheinlichkeitsbe- 
trachtung wird nämlich meistens falsch gesehen. Die Möglichkeit ist der empirische Befund: „Es kann bei der Gentechnik etwas passieren“. Die Wahrscheinlichkeit betrachten wir nicht abstrakt: „Was sind die Gefahren und die Risiken der Gentechnik?", sondern die Wahrscheinlichkeit betrachten wir konkret so: "Wie wahrscheinlich ist es, dass die Schutzvorkehrungen im Zusammenhang mit gentechnischen Vorhaben nicht greifen?" Das sind unsere realen, der Wirklichkeit verpflichteten, Risikobetrachtungen. Wir ermitteln, „Wie kann es passieren, dass die Schutzvorkehrungen eines Kernkraftwerks geknackt werden?" „Steht etwa wieder eine Flutkatastrophe an? Wie wahrscheinlich ist es, dass so etwas passiert?" Damit sind wir aber wieder bei den normalen klassischen Gefahrenbegriffen, bei den Gefahrenkategorien angelangt, bei Stufen der Eintrittswahrscheinlichkeit. Und weil Sie schon die Gentechnik erwähnt haben: Das Gentechnikgesetz rechtfertigte keineswegs den Siegeszug des Risikobegriffs in der Dogmatik der 90er Jahre. Die gesetzlichen Regelungen beschränkten sich vielmehr auf die Übernahme von Risk-Konzeptionen aus dem medizinischen Bereich der Vereinigten Staaten. Daraus wurden vier Sicherheitsstufen gebildet, die man Risikostufen genannt hat. Bei näherem Zusehen lässt sich das Gentechnikgesetz nur sinnvoll interpretieren, wenn man den Begriff „Gefahr“ statt „Risiko“ setzt. Denn die erste Risikostufe ist die Risikostufe 1, d.h., die Stufe, bei der kein Risiko besteht. Jetzt möchte ich das Risiko bestimmen, bei dem kein Risiko besteht. Das ist gelinde gesagt schwierig. Deswegen meint Risikostufe 1 die Stufe, bei der nur ein Risiko als Möglichkeit eines Schadens besteht, aber das auf Grund normativer Entscheidung sanktionslos bleibt. Das ist eigentlich der exakte Unterschied zwischen Risiko und Gefahr: Gefahr ist ein normativer Begriff, eine Eingriffsschwelle. Risiko ist ein empirischer Begriff. Die klare Unterscheidung verschwamm durch den undifferenzierten Gebrauch des Begriffs „Restrisiko" und der „Risikovorsorge“, auf den ich aus Zeitgründen nicht eingehen kann. Deswegen nochmals vielen Dank, Herr Lepsius, dass Sie tendenziell zu den klassischen polizeirechtlichen Kategorien der Gefahr und des Risikos zurückgefunden haben.

Stoll: Meine Frage bezieht sich auf den Titel der Referate - „Ermöglichung oder Begrenzung von Innovationen“. Ich habe in beiden Referaten Ansätze dafür gefunden, die unseren Begriff der rechtlichen Risikoregulierung erweitern, indem sie in Tendenzen davon ausgehen, dass Innovation auch eine Methode der Bewältigung bestehender Risiken sein kann. Herr Scherzberg, Sie haben das in Ihren Thesen 12 und 13 etwas ausgeführt, und es würde mich freuen, mehr dazu zu hören. Ich habe aber noch eine Frage zu Ihrer These 9. Herr Scherzberg, Sie haben davon gesprochen, wie sich eine Gesellschaft einen Begriff von einem Risiko 
macht, das wir als soziales Konstrukt verstehen. Nach Ihrem Vortrag konnte man den Eindruck gewinnen, als seien Innovationen ein Risiko an sich. Dafür könnte man anführen, dass „Neues“ eben erst noch staatlich und gesellschaftlich analysiert, ,kontrolliert" und reguliert werden muss. Aber wie passt das dann zu der Vorstellung einer Risikobewältigung durch Innovation? Herr Lepsius, die gleich Frage nach dem risikobegründenden und dem risikobewältigenden Charakter von Innovation würde ich gerne auch Ihnen stellen - und denke da besonders an die These 14. Beide Referenten wollte ich fragen, ob sich aus der wichtigen Rolle, die der Staat bei der Innovation, u.a. durch Förderung von Forschung und Entwicklung und durch Anreize spielt, nicht auch eine Verpflichtung des Staates erwachsen kann, zur Risikobewältigung, etwa auch durch Begleitforschung beizutragen. Wenn man sich die Förderung der Gentechnik und der Nukleartechnologie in den letzten Jahrzehnten ansieht, wird man feststellen, dass letzteres erst in jüngerer Zeit an Gewicht gewonnen hat."

Vorsitzender: Mit Frau Kokott wird der erste Problembereich abgeschlossen, und es geht dann über zu den Konstituierungsaspekten.

Kokott: Herr Lepsius, Sie fordern, wenn ich Sie recht verstehe, die Rückkehr zum wahrscheinlichen Schaden - analog zu dem von Ihnen so gesehenen Europarecht. Jetzt die Frage: Impliziert das eine erhebliche Einschränkung des unter anderem auch völkerrechtlich anerkannten Vorsorgeprinzips?

Hoffmann-Riem: Den Ausführungen von Herrn Murswiek schließe ich mich ausdrücklich inhaltlich voll an. Ich möchte die beiden Referate jedoch von einer anderen Fragestellung her ansehen: Was sagen Sie uns über das Verhältnis von Recht und Realität? Dabei habe ich Herrn Scherzberg so verstanden, dass er uns Mut machen will, die Herausforderungen der Gegenwart offen und auch mit dem Risiko anzugehen, sich der Wirklichkeit zu stellen. Herr Lepsius will uns stattdessen Mut zur Reduktion der Komplexität bei der normativen Einbeziehung der Realität machen. Das ist eine traditionelle Strategie, die für das Recht und seine Durchsetzung bisher in vielem sehr erfolgreich war: Man kann die Juristen und die von ihnen verantwortete Rechtswissenschaft häufig nicht dadurch angreifen, dass man auf einen Widerspruch zwischen der allgemeinen Wahrnehmung der Realität und ihrer rechtsnormativen Wahrnehmung verweist, weil ja ein Teil dieser Realität durch normative Filterung ausgeblendet wird. Gesichert wird durch diese Unangreifbarkeit auch ein Autoritätsgefälle zu Nichtjuristen. 
Wenn wir aber die beiden Vorträge von Herrn Vesting und Frau Kokott gestern mit den Vorträgen von heute verbinden, dann müsste eigentlich deutlich werden, dass diese traditionelle Strategie der Ausfilterung von Realitäten selbst riskant ist, weil dadurch auch normativ Wesentliches übersehen werden kann. Recht zeichnet sich gegenwärtig vielfach durch neue und komplexe Problemlagen und durch ein Netzwerk unterschiedlicher Möglichkeiten des Umgangs mit Problemen aus, insbesondere durch das Zusammenspiel rechtlicher Instrumente verschiedener Art auf vielen unterschiedlichen, wenn auch einander zugeordneten Regelungsschichten mit pluralen und heterogenen Akteuren. Auf Netzwerkkonstellationen aber ist unser überkommenes Recht nicht eingerichtet. Eben hat Herr Lepsius uns zum normativen Nachverdichten aufgefordert. Bei einem Thema, das auch von internationalen und globalen, in ihrer Entstehung vernetzten Risiken handelt, fordert die Problemlösung die Respektierung solcher Vernetzungen. Diesen Weg schlägt Herr Lepsius aber nicht vor. Er blendet vielmehr durch Rückkehr zu traditionellen Vorgehensweisen Schwierigkeiten aus und begnügt sich zum Teil mit normativen Fiktionen über die Realität. Sein Vertrauen in das Parlament - das ja in einer Demokratie zur Legitimationssicherung unverzichtbar ist - gilt überraschenderweise nicht dessen volitiven und damit politischen Fähigkeiten, sondern seinen kognitiven. Dass letztere aber vielfach zur Wahrnehmung der vollen Realität nicht ausreichen oder durch strategisch taktische Überlegungen aus dem Feld geschlagen werden, wird wohl jeder bestätigen, der Parlamentsarbeit durch eigene Anschauung kennt.

Eindrucksvoll erscheint mir die These von Herrn Scherzberg, der Gesetzesvorbehalt müsse neu und zwar als Mittel zur Qualitätssicherung eingerichtet werden. Dann muss der Gesetzesvorbehalt mehr sein als ein Eingriffsvorbehalt. Er ist auch als Vorbehalt einer gesetzlichen Sorge dafür einzurichten, dass die Möglichkeit zum Freiheitsgebrauch in der Risikogesellschaft und unter Bedingungen hoher Vernetzungen gesichert wird. Unter Innovationsaspekten bedeutet dies die Vorsorge für Problemlösungen durch Gesetze, die einen Weg zwischen den Polen möglichst hoher Innovationsoffenheit und hinreichender Innovationsverantwortung bahnen. Ein freiheitsermöglichendes Recht kann nicht auf Steuerung verzichten. Dabei darf der Steuerungsbegriff nicht so missverstanden werden, wie es Herr Lepsius in seiner Kritik getan hat. In der Steuerungsdiskussion der neueren Rechtswissenschaft sagt niemand, es ginge um die „Sicherung der rechtlichen Beherrschbarkeit des menschlichen Verhaltens". Unter Nutzung eines pragmatischen Steuerungsbegriffs lautet die Forderung in Abkehr von einem nur hermeneutischen Verständnis der Rechtswissenschaft vielmehr: „Recht hat sich um das Bewirken von Wirkungen zu bemühen und es hat möglichst zu vermeiden, 
dass unerwünschte Wirkungen eintreten". Das ist eine traditionelle Aufgabe des Rechts. Unter heutigen, vielfach vernetzten Bedingungen aber muss sie anders bewältigt werden als früher.

Pitschas: Herr Vorsitzender, meine sehr geehrten Herren Referenten, in dem von Ihnen fortgesetzten Prozess normativer Nachverdichtung liegt so etwas wie die beginnende Ausdifferenzierung eines neues Teils des Verwaltungsrechts, nämlich des Risikoverwaltungsrechts. Wollte man sich dieser Meinung anschließen, dann muss man jedoch zuerst fragen, was unter dem Konstrukt „Risiko“ zu verstehen sei. Darüber haben wir mancherlei gehört, aber ich bin mir noch nicht sicher, ob wir überhaupt schon wissen, was den Unterschied zwischen Gefahr und Risiko ausmacht und woran der Gesetzgeber anknüpfen sollte, wenn er einen allgemein verständlichen Teil dieses Risikoverwaltungsrechts entwickeln wollte.

Ich empfinde deshalb in Bezug auf die Thesen von Herrn Lepsius eine entsprechende Reserve. Ich meine, er ist der Semantik erlegen: Gefahr, Risiko, Restrisiko - oder auch ein zweistufiger Risikobegriff - das sind alles Formeln, die uns eigentlich nicht weiterhelfen. Wenn ich etwas im Risikoverwaltungsrecht regeln muss und diese Bezeichnungen verwenden will, dann muss ich den Ausgangspunkt klären und Klarheit darüber fordern: „Was ist das Risiko? Bitte beschreib’ es in zwei Sätzen“. Daran können dann Rechtsfolgen anknüpfen.

Im Übrigen habe ich zwei konkrete Fragen zu konkreten Themen. Zunächst geht es um das Problem der Effizienz. Wenn man Risikoverwaltungsrecht als soziales Schutzrecht gegen Risiken verstehen und ihm ein an marktwirtschaftlichen Entwicklungsmöglichkeiten orientiertes Innovationsrecht entgegensetzen wollte, dann stellt sich doch die Frage, lieber Herr Scherzberg, ob man nicht für bestimmte unterschiedliche Risikobereiche einen differenzierten Effizienzbegriff einführen müsste. Denn mir scheint, für die Regelung von technischen Innovationsprozessen wäre das Gewicht der Effizienzüberlegungen anders zu bestimmen als etwa im Polizeirecht, dem eine andere Schutzrichtung innewohnt. So gesehen wäre wohl doch zu klären, ob man ihre einschlägigen Thesen für alle Risikofelder im Recht, im Gentechnikrecht wie im Arzneimittelrecht, im Atomrecht oder im Polizeirecht für aussagekräftig hält, oder ob man nicht $\mathrm{Ab}$ stufungen im Schutzumfang nach dem Grad der Innovation zulassen muss, die jeweils bewirkt werden sollen. Dadurch würde deutlich, dass man mehr innovative Prozesse ermöglichen will.

Meine zweite Frage geht zu dem Bereich der Prävention an beide Referenten. Ich meine, dass in einem künftigen Risikoverwaltungsrecht auch ein Anteil an Präventionsrecht enthalten sein müsste. Nun hatten 
Sie, Herr Scherzberg, Prävention im Risikorecht auf Art. 20a GG bezogen und von hier aus die grundrechtlichen Schutzpflichten als Grundlage für die Prävention im Risikorecht eingebracht. Darf ich fragen, ob es so etwas gibt wie eine Verpflichtung des Staates auf der Grundlage dieser Schutzpflichten, für Prävention, also auch für einen Regelungskomplex zur Prävention im Risikoverwaltungsrecht, zu sorgen? Und ich bitte Herrn Lepsius freundlicherweise um die Erläuterung seiner letzten These Nr. 35, in der er die „übertriebene Expansion des Präventionsgedankens auf utopische Regelungsziele" bemängelt. Aber ich denke, es ist sehr wichtig, dass dem Risikorecht ein Präventionsaspekt innewohnt. Wo ist denn der Umschlag zur Utopie?

Calliess: Herr Lepsius, Sie haben in Threm anregenden Referat - ich möchte fast sagen, in bewährter Manier - effektvoll gegen den Strich gebürstet, gegen das schwierige und hochkomplexe Risikorecht, das in den letzten zehn Jahren ja zunehmend dogmatische Strukturen entwickelt und gewonnen hat, wobei mich, das sei angemerkt, Ihre Trennung zwischen Wahrscheinlichkeit und Möglichkeit nicht überzeugt hat. Denn ich meine, dass die Möglichkeit als Merkmal der Risikovorsorge ein an die Wahrscheinlichkeit als Merkmal der klassischen Gefahrenabwehr anknüpfender Teil des Dreistufenmodells ist. Sie trennen überdies nicht zwischen dem Risiko auf der Tatbestandsseite und Risikovorsorge als zu ergreifende Maßnahme in Reaktion auf ein Risiko auf der Rechtsfolgenseite. Aber insbesondere möchte ich fragen: Was ist nun Ihre Lösung in Reaktion auf die unbestrittene Herausforderung des Rechts durch die sog. Risikogesellschaft? Ist es die Rückkehr zum polizeirechtlichen Gefahrenbegriff? Ich glaube, diese Rückkehr, so dogmatisch eindeutig sie wäre, löst die Probleme nicht. Ihr Aufruf am Schluss des Referats „Vertraut dem Gesetzgeber" - völlig richtig, aber machen Sie es sich da nicht ein wenig zu leicht und verkennen die verfassungsrechtlichen Rahmenbedingungen, die eine gesetzgeberische Entscheidung konturieren? Übersehen Sie dabei nicht eine Dimension von Freiheit, die in den grundrechtlichen Schutzpflichten verkörperte Freiheit von Risiken? Ich meine, dass jede gesetzgeberische Risikoentscheidung die verfassungsrechtlichen Vorgaben an die Freiheitsverteilung zu achten hat und daher zur Vermeidung von rechtsstaatlich bedenklichen Schutzlücken über die Gefahrenabwehr hinaus auf die Risikovorsorge nicht verzichten kann. Daran schließt sich auch meine Frage an das Referat von Herrn Scherzberg an. Müsste man aus diesem Grunde nicht auch die verwaltungsrechtlichen Strukturen des Risikorechts, die Sie überzeugend aufgezeigt haben, stärker verfassungsrechtlich konturieren, gerade um die in jeder Risikoentscheidung liegende Freiheitsverteilung grundrechtsadäquat zuzuordnen? 
Rengeling: Ich möchte zwei Aspekte ansprechen, die mir praktisch besonders bedeutsam erscheinen und konkret sind (das ist vielleicht auch nicht ganz unwichtig). Der erste Punkt ist der, dass Herr Lepsius sehr schön in den Thesen 32 bis 34 auf die untergesetzlichen Regelungen hingewiesen hat, und deren Bedeutung möchte ich unterstreichen. Insbesondere im europäischen Bereich sind diese meines Erachtens nicht hinreichend erkannt, obwohl sie in der Praxis eine immense Rolle spielen. Wir werden die lege lata in der Verfassung der Europäischen Union das Institut einer Durchführungsverordnung bekommen, die der deutschen Rechtsverordnung entspricht, und damit ist auch gesagt - und so heißt es im Verfassungsentwurf, dass die nicht wesentlichen Regelungen (was auch immer das sein mag, wir kennen die Diskussion aus dem deutschen Recht) in einer solchen Durchführungsverordnung stehen dürfen. Ferner möchte ich ergänzen, dass dort die Regelungsverfahren (Stichwort Komitologie) eine außerordentlich große Rolle spielen. Sie werden häufig unterschätzt. $87 \%$ der Gemeinschaftsgesetzgebung geschieht nicht im normalen Gesetzgebungsverfahren mit Verordnungen und Richtlinien, sondern in diesem Bereich. Wenn man hinzunimmt, dass der Vorgang des Anpassens an den Stand der Technik in eben diesen Verfahren erfolgt, dann kommt es hier zum Schwur, nämlich dazu zu bestimmen, was wir hier unter Gefahr, Risiko usw. zu verstehen haben. Da spielt, wenn ich das einmal so platt sagen darf, wirklich dann die Musik. Soweit der erste Punkt.

Der zweite Punkt, den ich ansprechen möchte, betrifft die Instrumente, jetzt in einem engeren Sinne, und hier wollte ich eigentlich auch einen kleinen Schwerpunkt legen. Herr Scherzberg hat dazu in These 13 etwas gesagt, nämlich dass hier eine Auswahlmöglichkeit bestünde. Ich darf auch dazu wieder hinweisen auf die europäische Ebene. Es gibt ein wahrscheinlich auf die Dauer nicht ganz unbedeutsames Papier der Kommission über sogenanntes Gutes Regieren, wo der Auswahl der Instrumente für den Umweltschutz und überhaupt für das Gemeinschaftsrecht eine große Rolle zugemessen wird, und zwar in voller Breite. Das wiederum bedeutet, dass unsere Instrumente, wie wir sie bisher im deutschen Recht schon kennen, vielleicht noch erweitert werden. Wir haben eine Vielzahl: Wir haben Ordnungsrecht, wir haben Selbstverpflichtungen, wir haben Abgaben verschiedenster Art, wir haben EMAS sprich Ökoaudit, wir haben demnächst Zertifikate, und wir haben noch die Möglichkeit (jedenfalls wird es in der Praxis so verfolgt), über die öffentlichen Aufträge sogenannte vergaberfremde Zwecke zu verfolgen, die praktisch für den Umweltschutz und die Steuerung auch nicht zu unterschätzen sind - vor allem können sie erhebliche Innovationen hervorrufen. Übrigens möchte ich hier Herrn Stoll beipflichten, dass die Berücksichtigung des Begriffs 
der Innovation ein bisschen kurz gekommen ist. In der interdisziplinären Forschung zum Umweltschutz, wo die Juristen vielleicht in der Gesamtpalette nur einen kleinen Prozentsatz ausmachen, spielt dieser Gesichtpunkt eine immense Rolle. Meine konkrete Frage zu diesen Instrumenten ist die: Wenn wir eine solch riesige Palette haben, gibt es eigentlich Überlegungen (oder müsste man nicht vielleicht welche anstellen, vielleicht sogar von Verfassungs wegen), ob der Beliebigkeit, der Auswahl und der Anwendung dieser Instrumente insgesamt eigentlich auch einmal Grenzen gesetzt sind?

Vorsitzender: Wir kommen jetzt zu einem Beitrag zum Stichwort Risikopsychologie, Herr Engel bitte. Anschließend folgt die Gruppe „Verschiedenes".

Engel: Ich will versuchen, Sie davon zu überzeugen, dass man ein $\mathrm{Ri}-$ sikoverwaltungs- und ein Risikoverfassungsrecht nicht ohne Rücksicht auf risikopsychologische Einsichten schreiben sollte. Die erste Frage ist schon: Besteht überhaupt Anlass zu ... seit gestern bin ich vorsichtig geworden mit dem Wort staatlich, also sagen wir, öffentlichem Handeln? Die erste Einsicht, die uns die Risikopsychologie vermittelt, und die in den Referaten auch vorkam, wird mit dem Begriff loss aversion bezeichnet. Wir fürchten uns mehr davor, etwas zu verlieren, als wir bereit sind, zu investieren, um etwas zu gewinnen. Das heißt, Chancen sind konstitutiv vernachlässigt gegenüber der Sicht auf die Gefahren. Es könnte gesellschaftlich nützlich sein, dass wir dagegenhalten und ein Risikorecht haben, das nicht nur Risiken beherrschen, sondern Risikoübernahme in der Gesellschaft erst ermöglichen will.

Zum zweiten, und an Herrn Lepsius gewandt, sagt uns die Risikopsychologie etwas darüber, wie wir Risiko definieren sollten. Ontologisch und erst recht rationaltheoretisch haben Sie natürlich unzweifelbar Recht. Die Möglichkeit lässt sich interpretieren als eine beliebig kleine Wahrscheinlichkeit. Psychologisch besteht aber ein gewaltiger Unterschied zwischen Sicherheit und Möglichkeit. Das Allais-Paradox hat das sehr früh gezeigt. Fast alle Menschen bewerten ein noch so kleines Risiko ganz anders als Sicherheit. Daraus lässt sich möglicherweise psychologisch sehr gut erklären, dass die Politik Dinge tut, von denen sie in Wahrheit weiß, dass sie gar nicht gehen. Das Recht macht Sicherheitsbehauptungen. Es übernimmt Verantwortung. Es sorgt dafür, dass sich die Aufmerksamkeit auf ganz andere Risiken richtet.

Dritte Frage: Suche nach den Instrumenten. Herr Lepsius, Sie sagen, wir sollen tunlichst individualisieren. Das ignoriert aber auch wieder das psychologische Problem der loss aversion. Aus der Perspektive der loss 
aversion ist das, was Sie gegeißelt haben, nämlich Naturalisierung und Vergesellschaftung, eine geniale Strategie. Sie verhindert nämlich die $\mathrm{Zu}$ rechnung des Risikos und erlaubt damit, gesellschaftlich auszuhalten, dass wir in einer riskanten Welt leben.

Letzte Frage: Was heißt das nun für das Verfassungsrecht? Nach dem, was ich bis jetzt gesagt habe, könnte man befürchten, dass die Politik risikoscheu ist. Sie würde dann zu wenig Risiken übernehmen, gemessen an dem, was aus einer wie auch immer gearteten normativen, etwa utilitaristischen, Perspektive als angemessen erschiene. Praktisch sieht es tatsächlich ja aber eher anders aus. Die Politik ist geradezu hungrig danach, Risiken an sich zu ziehen. Erklärungen für diese Beobachtung finden sich an ganz anderer Stelle. BSE war natürlich ein wunderbares Instrument, um endlich der Landwirtschaftslobby beizukommen. Der Irakkonflikt war wunderbar, weil endlich niemand mehr über BSE sprach. Vor allem wird die staatliche Risikoübernahme psychologisch aber den handelnden Politikern zugerechnet und nicht dem Staat. Juristisch übernimmt das Risiko aber der Staat als juristische Person. Wenn das nun langfristige Risiken sind, ist es fast risikolos für die handelnden Politiker, dem Staat ein Risiko aufzubürden. Durch das Auseinanderfallen der politischen und der juristischen Zurechnung erhalten die Politiker die Risikoübernahme nämlich praktisch kostenlos. Für sie selbst zählt, ob sich die Chancen verbessern, die nächste Wahl zu gewinnen. Diese Chancen sind in keiner Weise beeinträchtigt, wenn sich das Risiko viel später realisiert und vom Staat als juristischer Person getragen werden muss. Aus dieser Perspektive mag es also wirklich sein, dass die staatliche Freude an der Übernahme von Risiken der Kontrolle bedarf. Dafür sind aber nicht die Gründe maßgeblich, die Sie uns vorgeführt haben, Herr Lepsius, sondern politökonomische Gründe.

Meyer (Zwischenfrage): Herr Lepsius, ich verstehe nicht ganz, wie Sie Ihre letzte These, dass das Risikoverwaltungsrecht jedenfalls nicht den Menschen selbst zum Risiko erklären soll, vereinbaren wollen mit dem Insistieren darauf (These 21), dass nur eine individuelle Zurechnung möglich sein soll. Und in These 25 rekurrieren Sie auf den Störer, der in der gesetzlichen Nachverdichtung sozusagen klarer herausgearbeitet werden soll. Müssten Sie nicht eigentlich zum Ergebnis kommen, dass der Mensch das Grundrisiko überhaupt ist und dass unser Problem darin besteht, dass dieses Grundrisiko zunächst einmal von der Verfassung erlaubt wird, weil die Freiheit des Menschen ja ein wesentlicher Bestandteil der Verfassung ist und unser Problem ja nur darin besteht, wie weit diese Freiheit gehen kann, oder umgekehrt, wann der Staat sagen kann: Dieser Freiheitsgebrauch ist unter Risikogesichtspunkten nicht mehr erlaubt? 
Albers: Meine Frage geht an Herrn Lepsius. Ich habe in Ihrem Vortrag die Beispiele vermisst, an denen sich die Plausibilität Ihrer Thesen messen lassen müsste. Ich möchte dazu einen zentralen Punkt herausgreifen. Das Risikorecht hat sich mit Blick auf die sogenannten Katastrophenpotentiale entwickelt, für die die Kernkraftwerke das klassische Beispiel sind. Wir wissen heute, dass sich dieses Problem nicht nur auf bestimmte großtechnische Anlagen, sondern etwa auch auf kumulierte Gewässerbelastungen, auf Waldschäden, oder, wie Herr Calliess schon anführte, auf den Klimawandel erstreckt. Mit Blick auf diese anschaulichen Phänomene war für das Recht erkennbar, dass es ein so differenziertes und mit vielfältigen Instrumenten arbeitendes Risikoverwaltungsrecht entwickeln muss, wie Herr Scherzberg uns das sehr schön vorgeführt hat. Kann man solche Probleme, wenn Sie jetzt diese Beispiele nehmen, wirklich mit dem doch ganz anderen Konzept der normativen Nachverdichtung der ordnungsrechtlichen Gefahrenabwehrdogmatik überhaupt erfassen oder sogar lösen? Ich habe da Zweifel in grundsätzlicher Hinsicht und auch bei vielen Ihrer detaillierteren Ausführungen. Wenn Sie etwa Erfahrungswissen durch Experimente gewinnen wollen, dann kann das bei Kernkraftwerken wohl kaum die Lösung sein.

Winter: Ich würde gerne mit einem Fall anfangen: Zwischen Bremen und Bremerhafen liegen ungefähr $50 \mathrm{~km}$, und nun telefoniert eine zunehmende Menge von Personen in Bremen und Bremerhafen mobil. Das führt dazu, dass die gesammelten Signale in Bremen nach Bremerhafen transportiert werden sollen und umgekehrt, und hierfür soll ein Richtfunkturm, etwa $100 \mathrm{~m}$ hoch, in der Mitte zwischen den beiden Städten gebaut werden. Die Anwohner haben Besorgnis wegen möglicher nichtionisierender Strahlen, und es gibt auch ein gewisses Problem mit dem Landschaftsbild. Was tut man jetzt? Wir haben hier die Situation der ungewissen Risiken. Man weiß noch nicht viel Bescheid, man weiß noch nicht viel über die Strahlenschäden. Nun ergibt sich aber, dass zwischen Bremen und Bremerhafen bereits ein Glasfaserkabel liegt. Der Turm soll von Vodafone gebaut und betrieben werden, das Glasfaserkabel wurde von Telecom verlegt. Telecom bietet an, die Signale durch das Glasfaserkabel zu schicken. Damit baut man keinen Turm. Ist es nun rechtlich sinnvoll, diese Frage der alternativen Zielverwirklichung - man könnte auch sagen, die Frage des Nutzens, die sich hier stellt - in dem Verfahren mit einzubringen, oder den Turm rein umwelt- und planungsrechtlich zu beurteilen? Ich lasse mal außen vor, dass wir da gewisse Hinweise in $\S 35$ Abs. 1 Nr. 3 Baugesetzbuch finden, das sollten wir einmal außer Betracht lassen. Mich interessiert die grundsätzlichere Frage, ob in der Risikoentscheidung eine Nutzen- und Alternativenerwägung eine Rolle spielen 
sollte, und zwar nicht nur in Zusammenhängen, in denen die öffentliche Hand - wie etwa bei Infrastrukturmaßnahmen - gefordert ist, Alternativen vorzustellen, sondern auch in solchen Verfahren, in denen Private tätig werden, wie Vodafone. Herr Hoffmann-Riem hat einmal einen schönen Aufsatz geschrieben über die Optionenprüfung, das Optionenermessen, und Herr Scherzberg hat in der These 26 die Figur der ökologischen Erforderlichkeit in die Debatte gebracht. Und hierhin richtet sich jetzt meine Frage, und mich würde auch interessieren, was Herr Lepsius dazu sagt: Wie würden Sie dieses Gebot dimensionieren? Erstens: Würden Sie den privaten Betreibern abverlangen, dass sie Alternativen vorstellen, und sagen, sie hätten sie geprüft, und die hätten sich nicht als sinnvoll erwiesen? Zweitens: Wie können Sie vermeiden, dass wir durch solch eine Risiko-Nutzenabwägung zu Situationen kommen, dass ein deutlicher Schaden über eine Nutzenprüfung hinnehmbar gemacht wird? Drittens: Wie ist es mit den europarechtlichen Bezügen? Im Europarecht gibt es zunehmend die Kategorie des hinnehmbaren Risikos, neuere Entwicklungen im Gentechnikrecht und im Chemikalienrecht, ich empfehle da, die neueren Rechtsakte dazu zu lesen, die Entwürfe. Und letzte Frage: Was ist die verfassungsrechtliche Grundlage einer solchen möglichen ökologischen Erfoderlichkeitsprüfung? Sind das Grundpflichten (Sie sprechen von Art. 20a), und wie ist dann das Verhältnis zu den Freiheitsrechten zu beurteilen?

Schoch: Verehrte Kolleginnen und Kollegen! Wer sich mit einem solch anspruchsvollen Thema beschäftigt, muss sich - um es bewältigen zu können - zunächst der Bedingungen im Realbereich vergewissern. Es geht dabei um Erkenntnisse, die aus dem Realbereich gewonnen werden, aber auch um Interessen, um Handlungsabläufe und um vieles anderes mehr. Sodann muss man die betreffenden Rechtsgebiete differenziert analysieren, um die jeweilige rechtliche Systemrationalität erfassen zu können; dabei muss man auch, was angesprochen worden ist, das Europarecht einbeziehen, und zwar nicht nur selektiv, sondern umfassend, um die richtige rechtliche Basis zu gewinnen.

Wenn man dies voraussetzt, dann kommt man - wobei ich zu zwei Punkten Stellung nehmen will - zunächst sehr rasch dazu, dass das uns hier angebotene Konzept der (generellen) administrativen Rechtsetzung die Lebenswirklichkeit in weiten Teilen ausblendet. Herr Rengeling hat bereits darauf hingewiesen, wie unter europarechtlichen Vorzeichen jenseits der selbstverständlich notwendigen Rechtsetzung - tatsächlich Entscheidungen in unserem Zusammenhang gewonnen werden. Ferner kann ich Ihnen berichten, dass die Entscheidung zum „Nanopulver“ des 10. Senats des VGH Baden-Württemberg, über die Herr Scherzberg be- 
richtet hat, selbstverständlich nicht in diesem relativ „blutleeren Raum“ der administrativen Rechtsetzung gewonnen werden konnte. Das angebotene Konzept der abstrakt-generellen Normsetzung formuliert notwendige, aber bei weitem nicht hinreichende Bedingungen für die Problembewältigung; darüber müssen wir uns Rechenschaft ablegen. Es genügt eben nicht, nur auf die differenzierte Rechtsetzung zu sehen; man muss auch den Verwaltungsvollzug einbeziehen und man muss die Erkenntnisse der Rechtsprechung in den Blick nehmen. Tut man dies, scheint mir das Zwischenfazit zu den Vorträgen eindeutig zu sein.

Was in den Vorträgen präsentiert worden ist, gibt - zweiter Punkt teilweise den Stand der Rechtswissenschaft nicht richtig wieder. Herr Hoffmann-Riem hat bereits darauf aufmerksam gemacht, dass der steuerungswissenschaftliche Ansatz wesentlich differenzierter ist, als aus diesen mitunter etwas "schwarz-weiß“ operierenden Argumentationsmustern deutlich geworden ist. Herr Lepsius, ich darf Sie direkt ansprechen und zugespitzt formulieren: Sie haben am Beginn und am Ende Ihres Referats eine ziemlich weit gehende Abrechnung mit Teilen der deutschen Staatsrechtslehre vorgenommen, indem Sie im Kern den Vorwurf erhoben haben, dass eine „Dogmatik des Unmöglichen“ entwickelt worden sei. Ich meine, ich habe Sie so richtig verstanden. Ihre Position wird am Ende Ihres Thesenpapiers nochmals in These 35 deutlich, wo Sie von „falschen Regelungserwartungen“ sprechen. Dazu möchte ich fragen, was Sie uns nun angeboten haben, und ich möchte genauso hart erwidern: Sie haben uns vor dem Hintergrund hochkomplexer Probleme einer ausdifferenzierten Rechtsordnung ein rechtliches Denken auf dem Stand des Kreuzberg-Urteils des Preußischen Oberwaltungsgerichts angeboten und gemeint, wir könnten hier mit den Rezepten des Polizeirechts arbeiten. Ich verstehe zwar, da ich mich mit dem Polizeirecht auch schon einmal befasst habe, dass Polizeirechtler in unseren Reihen von nostalgischen Gefühlen beschlichen werden und dem einfach strukturierten Konzept eine gewisse Sympathie abgewinnen können; ich fürchte nur, dass dieser Ansatz in unserem Zusammenhang nicht weiterführt. Ich möchte, wenn Sie erlauben, noch einen Schritt weiter gehen. Sie haben nicht nur einen „Frontalangriff“ gegen Teile der Staatsrechtslehre unternommen, sondern leider mit Ihrem Modell auch noch ein rechtlich nicht brauchbares Konzept geliefert. In These 27 sagen Sie, „Ziel muss es immer sein, Risiken zu individualisieren“. Nicht etwa „Beiträge“ zu einem Risiko, sondern „Risiken“ sind zu individualisieren. Ich frage, wie individualisieren Sie (und wie rechnen Sie zu) beispielsweise die Schädigung der Ozonschicht, die Verschmutzung der Meere und von mir aus auch die Schädigungen des Schwarzwalds? Darauf hätte ich gern eine Antwort. 
Vorsitzender: Ja, vielen Dank Herr Schoch für diesen grundsätzlichen Beitrag zum Begriff „Dogmatik des Unmöglichen“. Wir kommen jetzt zu den letzten beiden Wortmeldungen, und zwar diejenige von Herrn Streinz, der noch ein praktisches Beispiel uns vorführen möchte, und dem Beitrag von Herrn Meßerschmidt zur Frage der Beweislastumkehr.

Streinz: Zunächst etwas zum Europarecht und dann ein praktisches Beispiel. Von den beiden Referenten sind ja die europarechtlichen Vorgaben zutreffend einbezogen worden, insbesondere die Unterscheidung risk assessment, risk management und risk communication mit der zu Recht betonten Trennung von naturwissenschaftlicher Bewertung, mehr oder weniger based on science, und der politischen Entscheidung, auf deren Grundlage und im verfassungsrechtlichen Rahmen. Diese Trennung zeigt sich ja auch jetzt bei der Neuorganisation des Verbraucherschutzes in Deutschland nach der BSE-Krise. Aber dazu gehört auch die internationale Ebene, das möchte ich noch ergänzen, aus der diese Differenzierung stammt, im Rahmen der WTO. Hier zeigen sich ja deutliche Bewertungsunterschiede insbesondere zu den USA, wie die Fälle BST, d.h. Wachstumshormone, Novel Food, insbesondere hinsichtlich gentechnisch veränderten Organismen, zeigen. Normative Verdichtung ist hier besonders schwierig. Zu dem EuGH-Urteil und zur These 7 von Herrn Lepsius auch im Anschluss an die Frage von Frau Kokott: Wir haben hier das Problem, die Entscheidungen des Gerichtshofs zu systematisieren und zu generalisieren, die zu einzelnen Fällen ergehen und auf einzelne Fragen antworten. Die Tendenz sehe ich allerdings im Wesentlichen auch so. Nun das Beispiel, angeregt durch die These 20 von Herrn Lepsius und die These 25 von Herrn Scherzberg, nämlich der Verantwortungsteilung zwischen öffentlicher Steuerung und privatwirtschaftlicher Selbstregulierung - ein praktischer Fall, weil man sich ja oft die Frage stellt, ob unsere dogmatischen Erwägungen und Erkenntnisse auch zur Lösung praktischer Fälle beitragen können. Es geht hier um Folgendes - ich möchte es bringen, obwohl es kein innovatives, sondern ein traditionelles Produkt betrifft und banal erscheinen mag, aber von großer wirtschaftlicher Bedeutung ist: Es ist offenbar nicht auszuschließen und technisch auch nicht zu vermeiden, dass wegen - um es so zu umschreiben - natürlicher Umwelteinwirkungen in bestimmten Teebeuteln Salmonellen vorkommen können. Diese werden zuverlässig abgetötet, wenn der Tee mit heißem Wasser, in mindestens 70 Grad Celsius, aufgebrüht wird. Dass man dies machen soll, steht auch auf der Verpackung des Tees. Frage: Genügt dies, oder ist es so, dass wenn in einem Beutel Salmonellen festgestellt werden, die Regale geräumt werden müssen, wie die deutsche Verwaltungspraxis ist? Wenn man das deutsche Recht ansieht, $\S 8 \mathrm{LMBG}$, hat diese 
wahrscheinlich sogar Recht, wenn man das Gemeinschaftsrecht ansieht, Art. 14 EG-Basisverordnung, der am 1. 1. 2005 in Kraft tritt und der dem einzelnen Endverbraucher wohl mehr Eigenverantwortlichkeit zutraut, also auf dieser Stufe die Verantwortlichkeit ansiedelt, ist dies vielleicht anders zu sehen. Meine Frage daher: Wie würden Sie die Verantwortung teilen oder wem die Verantwortung hier zuteilen?

Meßerschmidt: Ich habe offensichtlich so unleserlich geschrieben, dass Sie meine Bemerkung nicht einordnen konnten. Entgegen meiner Gewohnheit muss ich eine kleine Vorbemerkung machen. Sich zu Risikoverwaltungsrecht zu äußern, ist offensichtlich selbst ein großes Risiko, auch eine Frage dazu zu stellen, denn man wird wohl immer eingeteilt: Gehört man zu denjenigen, die im Namen des Risikos Freiheit und Innovation erdrosseln wollen, oder gehört man zu denjenigen, die sozusagen auch unvertretbare Risiken hinzuzunehmen bereit sind. Ich glaube, beide Referenten haben dieses Risiko vermieden, auch wenn ihre Grundpositionen sehr unterschiedlich sind. Obwohl sich meine Fragen auf den Vortrag von Herrn Scherzberg beziehen, möchte ich doch nicht dem einen oder anderen Lager zugeordnet werden. Meine erste Frage bezieht sich auf die Beweislastverteilung bei Ungewissheitsrisiko. Ich habe Sie wahrscheinlich ein wenig einseitig verstanden, Herr Scherzberg, aber es klang so, als ob Sie es für möglich halten, dass der Hersteller eines Produkts oder eines chemischen Stoffes selbst beweisen muss, dass sein Stoff ungefährlich ist und dies selbst in der Situation des Ungewissheitsrisikos. Das liefe dann allerdings darauf hinaus, dass er im Grunde genommen gar nichts mehr machen kann, weil ein endgültiger Beweis der Ungefährlichkeit vielfach gar nicht zu führen ist. Wenn man so weit mit dem Begriff des Risikos geht und diese Koppelung von Beweislastumkehr und Ungewissheitsrisiko vornimmt, so scheint mir dies wirklich riskant zu sein. Ich glaube, mit dem Begriff des Ungewissheitsrisikos verträgt sich gerade keine eindeutige Beweislastverteilung. Aber vielleicht haben Sie ja auch mit Ihrem Abwägungsmodell eher in die andere Richtung weisen wollen. Das war mein erster Punkt, da bitte ich um Aufklärung, wie das zu verstehen ist. Der zweite Punkt: Wir kommen als Juristen ja nicht aus dem binären Kode heraus. Wir müssen uns festlegen: Was für ein Risiko ist es, ist es die Gefahr oder ist es das weniger schlimme Risiko? Wenn wir einen wissenschaftlich informierten Begriff und nicht nur eine opportunistische Ad-hoc-Dogmatik, wie das in der Vergangenheit in der Rechtssprechung der Fall war, verwenden, ergeben sich daraus Veränderungen bei der Zuordnung dessen, was wir bislang Gefahr und was wir bislang bloßes Risiko genannt haben? Kann es also sein, dass auf der Grundlage Ihrer Definition das, was wir bislang für bloßes Risiko gehalten haben, 
nun doch in den Gefahrenbereich abwandert oder kann es sich auch umgekehrt verhalten? Meine dritte Frage gilt den risikospezifischen Instrumenten: Ich habe da gewisse Zweifel, ob man immer sagen kann: Ordnungsrecht, das ist Gefahrenabwehr, und alle neuartigen Instrumente dienen der Vorsorge. Ich glaube, wir haben viele Instrumente, die sowohl eine Risikovorsorgekomponente besitzen als auch eine Gefahrenabwehrkomponente. Ich bin nicht sicher, ob es eine feste Relation zwischen Instrument und Schutzzweck gibt. Das müssten wir aber jedenfalls nicht nur für jedes Instrument, sondern bei jeder einzelnen gesetzlichen Regelung ganz genau prüfen.

Püttner (Zwischenfrage): Ich möchte nur Fragen - ich habe in den Referaten und in der Diskussion wenig gehört zur Risikofreudigkeit: Ist der Begriff mit Recht ausgeklammert worden, oder wie steht es damit?

Vorsitzender: Vielen Dank, das war wirklich ein kurzer Beitrag. Sie sehen mich, meine Damen und Herren, von der allerbesten Laune. Ich fand die Diskussion äußerst anregend, niveauvoll und auch von einer gewissen Konfliktbereitschaft gekennzeichnet. Damit sind wirklich zentrale Fragen angesprochen worden, und ich freue mich jetzt natürlich auf die Repliken der beiden Referenten. Wir werden so verfahren wie immer, dass der Zweitreferent beginnt, das passt von der Dramaturgie ja auch gut, weil Sie, Herr Lepsius, besonders viele Pfeile auf sich gezogen haben.

Lepsius: Danke, Herr Vorsitzender. Meine Damen und Herren, ich bedanke mich sehr herzlich für die vielen Fragen, kritischen Punkte, Anregungen, weiterzudenken, und bitte um Nachsicht, wenn ich nicht auf alle Aspekte mit der gebührenden Ausführlichkeit eingehen kann. Meines Erachtens sollten wir nach zwanzig Jahren Risikodogmatik eine Zäsur setzen, um nachzudenken: Was hat sich verändert, welche Entwicklungen sind passiert, wollen wir sie weitergehen, was soll auch in Europa vertreten werden, worauf sollten wir unsere Energien richten und in welchem Bereich sollte man vielleicht auch mal eine Innovation des Jahres 1980 dem Antiquitätenhandel übergeben? Die von mir als Zäsur angenommene Trennung von Möglichkeit und Wahrscheinlichkeit wurde angegriffen und „Vorbeireden an der Realität" genannt. Ich denke, es ist genau das Gegenteil. Wer die entscheidenden Schritte in der Rechtsentwicklung verfolgt, wird feststellen, dass diese Trennung immer wieder vorkommt, etwa in den Begriffsdefinitionen der UGB-Entwürfe. Das substanziell Neue dort ist der Möglichkeitsgedanke, der in die Risikodefinition einfließt, ebenso bei der Zielformulierung des $\S 1$ Gentechnikgesetz. Oder nehmen sie die Rechtsprechung des 7. Senats des Bundesverwaltungsge- 
richts aus den 1980er Jahren: In dem Moment, in dem der Senat die Möglichkeit in die Vorsorge einbezieht, muss er den Beurteilungsspielraum akzeptieren, weil er kein Kriterium zur Verfügung hat, um die Möglichkeit rechtlich zu überprüfen. Also immer wenn die Möglichkeit ins Spiel kommt, stehen wir vor großen Problemen. Daher ist diese Beschreibung nicht unterkomplex, sondern sie markiert die entscheidende Linie, an der die Rechtsprobleme beginnen.

In der Diskussion wurde gefordert, sich stärker mit dem Polizeirecht zu beschäftigen. Diese Tendenz finde ich unterstützenswert, nicht weil wir damit alle Probleme bewältigen können, sondern um uns der Grundlagen der Zurechnung bei der Gefahrenabwehr wieder zu vergewissern. Dies verstehe ich auch als Einwand gegen die Ausdifferenzierung weiterer Sonderbereichsdogmatiken. Es ist nicht hilfreich zu versuchen, die Komplexität der Rechtsentwicklung in ihren Verästelungen abzubilden, wenn die systembildenden Faktoren und die Grundlagen, von denen sich diese Sonderbereichsdogmatiken abwenden, in demselben Maße vernachlässigt werden. Wir stehen daher, glaube ich, vor einer Renaissance des allgemeinen Polizeirechts - gewiss mit der Einsicht, dass dieses alleine nicht reichen wird. Ich hoffe, das auch mit hinreichender Deutlichkeit im Vortrag gesagt zu haben. Aber dies sollte der Ausgangspunkt der Entwicklungen sein, und ich bin immer überrascht festzustellen, wie viele polizeirechtliche Grundprobleme seit Bill Drews noch ungeklärt sind. Der Begriff der Wahrscheinlichkeit ist in der Tat ein wichtiger Merkposten.

Wir sollten aber nicht nur das Verhältnis des Polizeirechts zu anderen Bereichen des besonderen Verwaltungsrechts bedenken. Auch das Verhältnis von Recht und Politik bedarf einer Besinnung und Rückversicherung. Ich wiederhole: wir trauen der Politik zu wenig zu, wenn wir nach der Verrechtlichung von gesellschaftlichen Problemlösungsinstanzen suchen. Wir haben diese Instanzen bereits: Es sind die Parlamente. Warum wollen wir die Funktionen des Parlaments mit den Mitteln des Verwaltungsrechts mühsam nachbilden? Warum wollen wir beispielsweise das Verwaltungsrecht mit partizipativen Strukturen überfordern, obwohl wir eine ganze Institution dafür unterhalten, diese partizipativen Strukturen zu erbringen? Ehrlich gestanden sehe ich darin keine Innovation. Auch inhaltlich wird das Parlamentsgesetz in seiner Problemlösungskompetenz unterschätzt. Natürlich kann der Gesetzgeber die Probleme des Umweltund Technikrechts regeln. Schauen Sie in die Vereinigten Staaten: Ich möchte die Detailfreudigkeit der Gesetzgebung dort nicht als Vorbild darstellen, aber dass der Gesetzgeber spezielle Regelungen im Umwelt- und Technikrecht vornehmen kann, verdeutlicht jeder Blick in amerikanische Gesetzbücher. Wir sollten uns hier auch von einem gewissen deutschen Traditionalismus verabschieden. Im Übrigen billigen wir dem Gesetzge- 
ber ja sogar zu, dass er Planfeststellungsbeschlüsse erlassen kann. Also wer die Südumfahrung Stendals kognitiv bewältigt, der kann auch noch ganz andere Dinge. Die Frage ist allerdings, warum wir bei bestimmten Sachfragen so leicht einknicken und sagen: „Nein, das kann der Gesetzgeber nicht". Warum geben wir uns eigentlich zufrieden mit $\S 7$ Atomgesetz oder mit § 5 Bundesimmissionsschutzgesetz? Ich sehe keinen Grund, in bestimmten Sachbereichen auf die verfassungsrechtlich gebotenen Anforderungen des Gesetzesvorbehalts zu verzichten. Der Gesetzesvorbehalt funktioniert in allen Bereichen, in denen er wirklich ernst genommen wird: etwa im Strafvollzugsrecht oder, wie die letzten Tage gezeigt haben, im Schulrecht. Ich sehe keinen empirischen, keinen rechtsvergleichenden, keinen kognitiven, ich sehe überhaupt keinen Grund, warum der Gesetzesvorbehalt nicht auch im Bereich des Umwelt- und Technikrechts ernst genommen werden sollte und der Gesetzgeber dann auch mit den Mitteln des Verfassungsrechts dazu aufgefordert werden müsste, die Dinge zu regeln. Wenigstens die Konkretisierungsdichte einer Landesbauordnung dürfen wir auch im Umwelt- und Technikrecht erwarten.

Dann ist nach dem Einfluss der Schutzpflichten gefragt worden, also dem Verhältnis des Verfassungsrechts zum Verwaltungsrecht. Zu den Schutzpflichten habe ich eine skeptische Position. Die Schutzpflichten haben wir seit 1975 und danach begannen die Schwierigkeiten! In dem Moment, in dem die Gefahrenabwehrdogmatik Schutzpflichten bewältigen musste, beginnt die Überforderung der Verwaltungsrechtsdogmatik, auch wenn die Überforderung in der Rechtsprechung des 7. Senats elegant gelöst wurde. Wir sehen zugleich die Überforderung der Gesetzgebung und auch ein wenig die Überforderung unserer eigenen Problemlösungskompetenzen. Ich sehe keinen Gewinn darin, diese Richtung weiterzuführen. Die Schutzpflichtendimension der Grundrechte ist vielmehr kritisch zu befragen, was sie eigentlich in den letzten 25, 30 Jahren eingebracht hat. Ich glaube, sie hat mehr Probleme verursacht als gelöst. Wir haben auch gar kein Bedürfnis für die Schutzpflichten, weil der Förderzweck, der Schutzzweck, entweder schon im grundrechtlichen Gesetzesvorbehalt selbst angelegt ist, nämlich in einem parlamentarisch zentrierten Gesetzesvorbehalt, der nicht nur der Eingriffsrechtfertigung dient, oder aus dem Sozialstaatsprinzip folgt. Mich interessiert, warum wir weder das Sozialstaatsprinzip noch den Gesetzesvorbehalt für die Schutzdimension heranziehen, sondern diese Frage über die Grundrechte konstitutionalisieren. Das ist, glaube ich, auch die staatsorganisatorisch falsche Verankerung. Wir überantworten der Grundrechtsdogmatik zu viel und überfordern sie hier bei der Problemlösung.

Das Problem des Risikos stellt das Verwaltungsrecht vor eine sehr interessante Herausforderung, nämlich vor die Herausforderung, seine Gren- 
zen zu thematisieren. Man ist vielleicht versucht, über den Vorsorgegedanken, der ja auch in der Tradition der Daseinsvorsorge steht, die Grenzen auszudehnen, und ich habe durchaus provokativ auf diese Grenzen hingewiesen. Das Verfassungsrecht ist dem Verwaltungsrecht insofern überlegen, weil es die Debatte nach seinen Grenzen schon geführt hat (etwa am Beispiel der sozialen Grundrechte oder des Sozialstaatsprinzips). Das Verwaltungsrecht hingegen muss die Debatte über die Grenzen seiner Leistungsfähigkeit erst noch führen. Momentan traut es sich zuviel zu, wenn es meint, verfassungsrechtliche Aspekte erneut abbilden zu können.

Damit komme ich zum letzten Punkt, dem Verhältnis des nationalen Rechts zum Europarecht. Vielleicht kommt die Lösung vieler Probleme doch aus Europa, und wir sollten europarechtliche Entwicklungen nicht nur als Verfallserscheinungen thematisieren. Gerade im Bereich der Gefahrenabwehr empfand ich die europarechtlichen Einflüsse als wohltuend. Angelsächsischer Pragmatismus führt uns vielleicht eher auf den Boden der Tatsachen zurück als deutscher Idealismus, der natürlich im Unterricht an anderer Stelle zu seinem Recht kommen sollte, aber vielleicht nicht im Umweltrecht. Das führt mich zu einer letzten Bemerkung: Wir gehen, glaube ich, zu leichthändig von der Auffassung aus, dass deutsche Ideen, wenn sie denn nach Brüssel exportiert werden, als deutsche Ideen zurückstrahlen. Der Begriff der Vorsorge, der ja eine deutsche Erfindung ist und auf deutsches Betreiben ins Primärrecht eingeführt wurde, kommt aus Europa anders zurück, als er dorthin geschickt wurde, und für das Verursacherprinzip kann man nur das Gleiche hoffen. Jedenfalls beobachten wir Entwicklungen mit interessanten Ergebnissen, und ich meine, wir sollten sie als Chance begreifen, uns von manchen Entwicklungen der letzten 20 Jahre auch wieder zu befreien.

Scherzberg: Meine sehr verehrten Damen und Herren. Leider kann ich aus Zeitgründen nur zu einigen zentralen Punkten der heutigen Diskussion Stellung nehmen. Ausgangspunkt ist dabei für mich die Feststellung, dass das Recht die Aufgabe hat, die Probleme zu bearbeiten, die die gesellschaftliche Wirklichkeit ihm stellt. Die Risikogesellschaft stellt dem Recht ein besonderes Problem: in ihr geht es um die Beurteilung von Produkten, Verfahren und Anwendungen, deren Nutzen regelmäßig bekannt, deren Nebenfolgen aber ebenso regelmäßig unbekannt sind. Die rechtliche Bewältigung dieser Lage kann mit dem klassischen Instrument der Abwägung von Kosten und Nutzen - etwa im Rahmen des Verhältnismäßigkeitsprinzips - allein nicht gelingen. Denn es stehen sich nicht Kosten und Nutzen oder Sicherheit und Unsicherheit, sondern relative Sicherheit der Nutzen mit Ungewissheit der Nebenfolgen gegenüber. Das stellt das Recht vor ein neues Problem. 
Bislang konnte man Ungewissheit im Reich der Gefahrenabwehr durch Rückgriff auf das Erfahrungswissen des Vollzugsbeamten lösen. Erfahrungswissen gibt es aber zu den meisten Regelungsproblemen der Risikogesellschaft nicht. Nehmen Sie etwa ein Holzschutzmittel: Das Holzschutzmittel beugt Fäulnis vor - das weiß man, das hat man untersucht, deshalb ist es auf den Markt gebracht worden - und es sichert damit die Stabilität der Dachkonstruktion. Aber wir wissen nicht genau, welche weiteren Wirkungen der Kontakt mit Holzschutzmittel im menschlichen Körper auslöst. Ein anderes Beispiel ist der gentechnisch veränderte Mais. Er erspart die Nutzung von Pflanzenschutzmitteln, aber wir wissen noch nicht genau, wie er auf die Bakterien im menschlichen Darm wirkt. Wir wissen nur, dass er dort wirkt. In diesen Fällen bedarf es einer Bewertung des Risikos, und zwar, Herr Püttner, auch der Risikofreude. Die Aufgabe dieser Bewertung hat natürlich - da stimme ich meinem Kollegen Lepsius durchaus zu - primär der Gesetzgeber. Er hat über die Risikobereitschaft der Rechtsordnung zu entscheiden. Er muss dabei aber auch ein Instrumentarium nutzen, das dem Faktum gerecht wird, dass wir es mit einer ungewissen Situation zu tun haben. Der Gesetzgeber muss also von der erfahrungsbasierten Steuerung auf eine ungewissheitsbasierte Steuerung übergehen. Man kann dabei nicht mit alten Konzepten und Begrifflichkeiten des Polizeirechts arbeiten. Wenn man das versucht, Herr Ronellenfitsch, endet man in einem Zirkelschluss. Ich möchte Ihnen das am Beispiel der Abgrenzung von Vorsorge und Restrisiko vorführen. Auf der Basis der klassischen polizeilichen Unterscheidungen soll in Fällen der Ungewissheit bei potentiell irreversiblen Schäden bereits eine geringe Prognosesicherheit für die Gefahrenannahme ausreichen. Nicht etwa eine geringe Wahrscheinlichkeit, sondern eine geringere Prognosesicherheit. Dabei wird übersehen, dass die Annahme der Irreversibilität des Schadens selbst auf der betreffenden schwachen Prognosebasis beruht. Die Anwendung klassischen Polizeirechts auf Fälle der Ungewissheit führt mithin in einen Zirkelschluss.

Das Recht braucht neue Instrumente zur Ungewissheitsbewältigung. Das wichtigste ist dabei, dass es reflexiv wird. Man muss sich vergegenwärtigen, dass das Recht selbst Risiken schafft, wenn es in ungewissen Tatsachenlagen reguliert. Das ist in der Diskussion verschiedentlich zutreffend vermerkt worden. Ich würde die Notwendigkeit, die Folgen des Handelns unter Ungewissheit $\mathrm{zu}$ berücksichtigen, verfassungsrechtlich primär in Art. 20 a GG verorten, aber - Herr Pitschas - auch in den grundrechtlichen Schutzpflichten, und zwar in der Art, dass die Schutzpflichten zur Risikobegleitforschung verpflichten, wenn das Recht ein grundrechtlich relevantes Risiko eröffnet, und zwar auch ein Restrisiko. Die Risikobegleitforschung kann entweder der Staat selber unternehmen 
oder dem Betreiber auferlegt. Bei der Berücksichtigung des Risikos zweiter Ordnung, also der Gefahr der Fehlsteuerung der Gefahr, muss man natürlich die in Frage stehenden Innovationen gewichten und den möglichen Wert der Innovation einstellen, und deswegen kann man bei der Risikosteuerung durchaus graduell abgestuft nach Maßgabe des erwarteten Nutzens der Innovation agieren, wie ein Diskussionsteilnehmer zu Recht gefragt hatte.

Ich möchte als Beispiel noch mal den VGH Mannheim mit seinen Nanopartikeln anführen. Es gibt für Nanotechnologie noch keine Grenzwerte. Deswegen hilft hier auch der Ruf nach dem Gesetzgeber nichts. Die Behörden knüpfen stattdessen an den Grenzwert für Dieselruß an und setzen dazu einen Sicherheitszuschlag von zwei Zehnerpotenzen. Warum nicht drei Zehnerpotenzen, warum nicht vier, warum überhaupt einen Sicherheitszuschlag? Es gibt keine Sachverständigen, die dazu eine rationale, sichere Antwort geben würden. Deshalb stellt sich hier der Verwaltung die Aufgabe, ein Risikokonzept zu entwickeln. Sie muss dabei auch den zur Umsetzung gesetzlicher Schutzvorgaben angemessenen Sicherheitszuschlag bestimmen. Die Gerichte überprüfen diese Entscheidung dann nur auf ihre Vertretbarkeit. Und so ist es in der Tat in den Vorsorgeerwägungen der VGH auch geschehen.

Ein letzter Punkt, den ich ansprechen möchte, betrifft die Wahrscheinlichkeit. Ich bin kein Wahrscheinlichkeitstheoretiker, aber ich muss Ihnen sagen, ich glaube nicht an die rationale Kraft dieses Begriffs. Zum einen deswegen nicht, weil man in Ungewissheitssituationen die Wahrscheinlichkeit regelmäßig nicht kennt. Die Wahrscheinlichkeit wird fingiert. Sie entsteht aus Extrapolationen, Modellrechnungen, Simulationen, die sich häufig genug in der Wirklichkeit als ungenau oder unrichtig erweisen. Zum anderen deshalb, weil sich der Wahrscheinlichkeitsbegriff außerhalb sich regelmäßig wiederholender Ereignisse für die Risikosteuerung überhaupt nicht eignet. Denn wenn man in die Welt hineinschaut, dann ist sie voll von unwahrscheinlichen Ereignissen. Dass sich die Mitglieder der deutschen Staatsrechtslehrervereinigung so auf ihre Stühle in diesem groBen Hörsaal zuordnen, wie sie sich zugeordnet haben, das ist höchst unwahrscheinlich und doch genau so eingetreten. Und wenn Sie sich vorstellen, meine Damen und Herren, dass jeder von Ihnen das Produkt der Rekombination des Erbmaterials Ihrer Eltern sind und es dabei drei Milliarden verschiedener Buchstaben gibt, die da kombiniert werden müssen, dann müsste man Sie, meine sehr verehrten Damen und Herren, wenn man an Wahrscheinlichkeiten glaubt, allesamt nach praktischer Vernunft vernachlässigen. Man darf, wenn man in die Zukunft schaut, nicht einfach die wahrscheinlichen Entwicklungen erwarten, sondern muss mit dem Eintritt des Unwahrscheinlichen, des Möglichen eben, rechnen. 
Vorsitzender: Vielen Dank noch einmal an die Referenten für die unwahrscheinlich guten Referate und für die inhaltsreiche Diskussion. Wir machen jetzt unsere verdiente Mittagspause und treffen uns um 14.15 pünktlich zur Fortsetzung unserer wissenschaftlichen Diskussion. 